\title{
Constructing an understanding of mind: The development of children's social understanding within social interaction
}

\author{
Jeremy I. M. Carpendale \\ Department of Psychology, Simon Fraser University, Burnaby, BC, V5A 1S6, \\ Canada. \\ jcarpend@sfu.ca \\ http://www.psyc.sfu.ca/people/index.php?id=67
}

\author{
Charlie Lewis \\ Department of Psychology, Lancaster University, Lancaster, LA1 4YF, \\ United Kingdom. \\ c.lewis@lancaster.ac.uk \\ http://www.psych.lanc.ac.uk/people/CharlieLewis.html
}

\begin{abstract}
Theories of children's developing understanding of mind tend to emphasize either individualistic processes of theory formation, maturation, or introspection, or the process of enculturation. However, such theories must be able to account for the accumulating evidence of the role of social interaction in the development of social understanding. We propose an alternative account, according to which the development of children's social understanding occurs within triadic interaction involving the child's experience of the world as well as communicative interaction with others about their experience and beliefs (Chapman 1991; 1999). It is through such triadic interaction that children gradually construct knowledge of the world as well as knowledge of other people. We contend that the extent and nature of the social interaction children experience will influence the development of children's social understanding. Increased opportunity to engage in cooperative social interaction and exposure to talk about mental states should facilitate the development of social understanding. We review evidence suggesting that children's understanding of mind develops gradually in the context of social interaction. Therefore, we need a theory of development in this area that accords a fundamental role to social interaction, yet does not assume that children simply adopt socially available knowledge but rather that children construct an understanding of mind within social interaction.
\end{abstract}

Keywords: language; Piaget; social interaction; theories of mind; Vygotsky; Wittgenstein

\section{Introduction}

Debate concerning how children come to understand the social and psychological world, now often known as children's "theories of mind," has become increasingly concerned with the influence of social interaction. Recent studies have found that individual differences in preschoolers' competence on measures of false belief understanding are correlated with aspects of children's socialization history. By suggesting that social interaction may influence the development of children's mentalistic understanding, this research has reopened issues that have long been discussed in the debate over how children come to understand the mind.

As an example of this research, Perner et al. (1994) reported that preschoolers with siblings demonstrate false belief understanding at an earlier age than children without siblings. This "sibling effect" was replicated by Jenkins and Astington (1996), although they found it to be less pro- nounced for children with more advanced linguistic abilities. Lewis et al. (1996) also found in one study an association between number of siblings and performance on false belief tests, but, overall, they found a more consistent effect of older siblings and kin on the development of false belief understanding. In a series of experiments with a large number of participants, beneficial effects were found for older but not younger siblings (Ruffman et al. 1998). The sibling effect was not replicated, however, in two more recent studies involving working-class families (Cole \& Mitchell 2000; Cutting \& Dunn 1999). This research suggests the possibility that, rather than just the number of people in the household, it is the nature of the relationships children experience that influences development (Hughes et al. 1999). There is further evidence of correlations between social cognitive development and parenting style (Hughes et al. 1999; Ruffman et al. 1999; Vinden 2001), aspects of parent-child conversation (Sabbagh \& Callanan 1998), attachment (Fonagy et al. 1997; Meins 1997; Meins 
et al. 1998; Symons \& Clark 2000), mothers' education (Cutting \& Dunn 1999), and socioeconomic circumstances (Holmes et al. 1996).

Furthermore, a number of studies have found correlations between language and social understanding (e.g., Cutting \& Dunn 1999; de Villers 2000; Happé 1995; Jenkins \& Astington 1996). In longitudinal studies, forms of family talk about mental states have been found to be related to later success on false belief tests (e.g., Brown et al. 1996; Dunn et al. 1991a; Moore et al. 1994; Ruffman et al. 2002). In addition, mothers who think of their children in mentalistic terms ("mindmindedness"), and therefore, presumably talk to their children about the psychological world, have children who are more advanced in understanding beliefs than are other children (Meins \& Fernyhough 1999; Meins et al. 1998). Similar correlations between family interaction and the development of children's understanding of emotions have also been reported (e.g., Dunn et al. 1991b; Hooven et al. 1995; Kuebli et al. 1995; Steele et al. 1999). In a longitudinal study, Astington and Jenkins (1999) found that earlier language abilities predict later false belief performance but earlier false belief competence does not predict later language abilities, supporting the conclusion that language is important in social cognitive development.

Another source of evidence that social interaction is important for the development of a mentalistic understanding comes from research with deaf children. A number of studies have shown that deaf children with hearing parents are delayed in the development of false belief understanding, whereas deaf children with deaf parents are not delayed (Peterson \& Siegal 2000; Woolfe et al. 2002). This seems to be because deaf parents are native users of sign language and thus their children are exposed to normal conversation, but hearing parents are less fluent in sign language and

Jer emy I. M . Car pendal e, Associate Professor of Developmental Psychology at Simon Fraser University, has published in the areas of cognitive, social cognitive, and moral development. His work focuses on the nature and development of thinking about social and moral matters and the role of language viewed as activity and grounded in shared meaning in such development. He is the editor, with Ulrich Müller, of Social Interaction and the Development of Knowledge (2004, Lawrence Erlbaum Associates) and author, with Charlie Lewis, of How Children Develop Social Understanding (forthcoming, Blackwell).

Charlie Lew is, Professor of Family and Developmental Psychology at Lancaster University, conducts research on the family, especially the role of the father, and young children's understanding of mental states in a longstanding collaboration with Norman Freeman. He is the editor, with Peter Mitchell, of Children's Early Understanding of Mind (1994, Lawrence Erlbaum Associates). Work on this article was started when he won a Noted Scholar Award from the University of British Columbia in 1997. therefore their children are not exposed to complex conversation about everyday events involving people's actions, beliefs, and emotions. Conversation about the mental world may well be essential for the development of social understanding.

This accumulating evidence that aspects of social interaction are correlated with social understanding must be explained by a complete account of social cognitive development. The contemporary body of research in this area derives from one of the dominant theories according to which children come to understand their own and others' minds by formulating an implicit "theory" of the mind (Astington et al. 1988). In this article we will, in general, use the broader phrase, social understanding (Dunn 1988) as we present an alternative position. All theories of the development of social understanding have had to recognize the influence of social interaction on social understanding, but they do so in very different ways and most accounts still begin from an individualistic perspective. It has seemed that the only possible alternative is to contrast individualistic accounts with unspecified statements about the "enculturation" of the child. In this article we argue for an alternative account of the development of mental state understanding that integrates the social and individual dimensions of development. We contend that the child does not merely adopt socially available knowledge, but rather, within social interactions an understanding of mental states develops and is constituted. Our approach is based on Chapman's (1991; 1999) reformulation of Piagetian theory, drawing on Vygotsky and Wittgenstein, and it is also consistent in some ways with other contemporary approaches (Hobson 1993; 2002; Montgomery 1997).

To introduce our alternative theory, we set it in the context of continuing debates and competing theories within the "theory of mind" tradition (sect. 2). We first discuss the more general issue concerning the relative contributions of social versus individual processes in development (sect. 2.1 ), and then turn to attempts by competing theories to explain the role of social interaction in the development of children's social understanding (sect. 2.2). In the third section, we introduce our alternative constructivist approach in which we argue that social interaction is essential in the development of cognitive, social, and moral knowledge. We briefly discuss the development of infants' ability to engage in triadic interaction involving the self, others, and the physical world (sect. 3.1). This basic yet essential level of social understanding allows children to engage actively in social interaction and to acquire language, and thereby gradually to develop a more sophisticated mentalistic understanding by learning the criteria for the use of words referring to the mental world (sect. 3.2). In the fourth section, we address two important issues that distinguish our account from those we criticize. We explore what has been taken as the crucial evidence for the dominant theoretical approach and suggest that the explanatory framework presented here explains the data more completely (sect. 4.1). Not only does the constructivist perspective better account for the range of experimental evidence, it can also explain the role of relationships in social development, like the sibling effect and the influence of attachment patterns (sect. 4.2). We conclude (sect. 5) by making some suggestions for future directions in research that follow from our theoretical perspective. 


\section{The problem with the "theory of mind" tradition}

The problem with the "theory of mind" literature is that the majority of approaches to the issue are individualistic. This is usually contrasted with a straw person, referred to as enculturation. In the two parts of this section we first describe a solution to the individual/social dichotomy before articulating how the problem of individualism manifests itself in contemporary theories.

\subsection{Theory construction versus social construction: Individualism, collectivism, and relationalism}

At the broadest level, debate regarding the development of children's social understanding concerns the relative contributions of social and individual processes. This is the issue of whether theories start with the individual or focus on the influence of the social group on development. We first illustrate how this issue has emerged in research on the development of children's social understanding, and then set this debate in the context of the more general issue in order to derive an approach to resolve this problem.

In the context of social cognitive development, the developmental question, according to Raver and Leadbeater (1993), is "whether the true starting point is to be located in the single, isolated, free mind of the individual or in a social, communal world of shared experience or language" (p. 355). Raver and Leadbeater contrasted the "theory of mind" and social development research programs and suggested that

Theory-of-mind research focuses on the single mind of the individual child as a rational empiricist, processing incoming perceptual data and reporting the results of those observations. In contrast, social developmentalists focus on the interaction of at least dyads, and the development of social minds of children communicating in a peopled world. (Raver \& Leadbeater 1993, p. 355)

This tension has also been articulated by Astington and Olson (1995) as occurring between theory construction and social construction. They suggested that an alternative to theory construction in which "children construct a theory about human talk and action" (p. 185) is a process of enculturation in which "children internalize the folk psychology of their particular culture" (p. 184), and, therefore, an understanding of mind acquired in such a fashion is "a set of cultural norms" (p. 185). In this view, social construction is equivalent to enculturation and the ability to "participate in a kind of interpretive discourse. ... In the one case the child is seen as constructing concepts, in the other as internalizing social understanding" (p. 185). Internalization, in this socialization approach, involves making external social norms internal. This enculturation approach, which has been attributed to Wittgenstein, was clearly stated by Astington and Gopnik (1991, pp. 19-20):

On this view folk psychology is . . . what Wittgenstein would call a "form of life," a set of social and cultural practices and conventions. The mechanism for development, on this view, would be socialization or enculturation - children would learn how to psychologize appropriately in the way that they learn to dress properly or eat politely,

or learn that "forks go on the left" (Gopnik 1993, p. 3). Astington and Gopnik (1991, p. 20) further specified the difference between the view they attributed to Wittgenstein and the "theory formation" view, by stating that theories always develop with reference to the outside world; put very simply, a theory former wants to get closer to the truth. Cultural practices, on the other hand, are, at least largely, selfconstitutive: they make themselves the case. Theories are true or false, cultural practices are right or wrong. ${ }^{1}$

The implication of this interpretation of Wittgenstein is that social cognition is relativistic and therefore forms of social understanding are culture-specific and free to vary across cultures, just as ways to dress properly or eat politely vary. We, however, argue in section 3.2 for a different interpretation of Wittgenstein. Briefly, although we acknowledge cultural variation (Lillard 1998; Vinden 1996; 1999), the basic forms of understanding of self and others that we are concerned with in this target article are built onto forms of shared practice or everyday activities that are necessarily part of human interaction, such as adults caring for infants, and are therefore likely to be common across cultures (Canfield 1993).

Astington and Olson (1995), as well as Raver and Leadbeater (1993), suggested the need for an integration of the two perspectives - that is, the isolated individual formulating a theory of social behavior versus the child passively adopting concepts available from the culture. The conflict between these two contrasting positions, often referred to as individualism and collectivism, is a general issue concerning the role of social interaction in the development of knowledge that arises in many areas of development (Carpendale \& Müller 2004). Given that he is often mislabeled as the archetypal "individualist" developmental theoretician, it might seem surprising that it was this "perennial problem" that Piaget (1977/1995, p. 184) grappled with in a series of essays published as the Sociological Studies (Chapman 1986; Kitchener 1986; Smith 1995). The question for Piaget was "Do the operations by means of which we attain what rational consciousness calls truth depend on society and, if so, in what sense?" (p. 184). He criticized individualism for neglecting the role of social life in transforming the individual's cognitive structures; in Piaget's words, "human knowledge is essentially collective, and social life constitutes an essential factor in the creation and growth of knowledge, both pre-scientific and scientific" (Piaget $1977 / 1995$, p. 30).

On the other hand, collectivism is also problematic for a number of reasons. One difficulty is that if knowledge is simply passed on from the collective to the individual, how is it that individuals can question and criticize collective beliefs? "If truth is something social, how can one distinguish legitimate common representations from collective beliefs not based on reason?” (Piaget 1977/1995, p. 197). Piaget also criticized collectivism for not considering the influence of different types of social relationships on development (Carpendale \& Müller 2004; Duveen 1997).

As an alternative to individualism or collectivism, Piaget argued for a third possibility, according to which it is relations between individuals that are primary (Kitchener 1986, Ch. 4; Smith 1995; see also Bunge 2000; Elias 1978): “The primary fact, from this point of view, is neither the individual nor the set of individuals but the relationships among individuals, a relationship constantly modifying individual consciousnesses themselves" (Piaget 1977/1995, p. 136). This position leads to a consideration of different types of relationships and their influence on development: "One will no longer be content to say that 'society' is the basis of 
logic but will ask exactly what social relationships are involved" (p. 136). The two types of relationships that Piaget (1932/1965b) described, forming the extremes of a continuum, were constraint and cooperation. Constraint involves inequality and views being imposed by authority, whereas cooperation involves interaction among equals.

Our approach to the development of children's social understanding focuses on the relations between people. From a relational, action-based perspective the developing child is embedded in social interaction, and an involvement in social activity itself is an integral part of constructing knowledge of this activity. This activity simultaneously involves operative interaction with the world as well as communicative interaction with other people (Chapman 1991; 1999). It is the mutuality of operative and communicative interaction that forms the basis of the constructivist position proposed here.

Although we have focused on Piaget, a relational, embodied, constructivist approach to development could also be based on other theorists (Bunge 2000; Elias 1978; Overton 1998b). A number of approaches recognize the importance of social interaction in development (e.g., Cole 1992; Rogoff 1997; 1998), but this is not the place to exhaustively review and critique them. ${ }^{2}$ The perspective we take is on the psychological development of infants and children with the assumption that such development occurs within the infant's or child's activity - an activity matrix made up of biological, social/cultural, and psychological dimensions (Overton 1994). From this perspective, development begins from a point of relative lack of differentiation, and the child's distinctions between self, other, and the world of objects emerge through activity in such a matrix. ${ }^{3}$

An issue that arises in regard to constructivism is that such approaches may appear to imply relativism. That is, if knowledge is constructed within social interaction, then, because social interaction may vary across cultures, it would appear that such knowledge would be specific to particular cultural groups. Moreover, there would be no way to evaluate different forms of knowledge; all forms would be equally valid. That is, there are just different forms of knowledge and there is no way to be able to say one is better than another. This "constructionist" position (Gergen 1994), therefore, collapses the distinction between development and mere change and amounts to relativism (for further discussion, see Chandler 1997). However, although the constructivist position we endorse accepts that knowledge is constructed within interaction, there is still development in the sense that one form of knowledge is better, more complete, or more adequate than other forms of knowledge (Chapman 1988b).

As a way to resolve this difficulty with relativism, Chapman (1988b) distinguished progressivity in development from directionality toward a predetermined end point. According to Chapman's (1988b) reading of Piaget's equilibration theory, this theory was meant to address the question of "how one form of thinking or knowing might be judged more or less 'advanced' than another" (p. 97). After the necessary first step of describing forms or stages of thinking, the next step for Piaget was to explain the process of development from one form of knowledge to another. Development from this approach is progressive in the sense that partial and incomplete perspectives are coordinated, resulting in more coherent and complete forms of knowledge. The process of equilibration is assumed to be univer- sal and progressive, but this does not imply a particular predetermined end-state. Rather, development involves movement away from an initial starting point of lack of knowledge (Chapman 1988b).

In the next subsection we discuss attempts to account for the influence of social interaction on the development of children's social understanding at the level of particular theories of children's social cognitive development.

\subsection{Accounting for the influence of interaction on children's social understanding}

The issue of how to address the social dimension in development is of importance within debates over which is the best theoretical account of children's understanding of mind, because each of the competing accounts must explain the recent evidence suggesting that social interaction influences such development. Until recently, the discussion concerning theories of children's "theories of mind" primarily consisted of debate among three dominant theories: theory-theory, modularity theory, and simulation theory. The pace and excitement of debate among these factions over the past decade has been frantic (for reviews see, e.g., Flavell \& Miller 1998; Hala \& Carpendale 1997; Lewis \& Carpendale 2002). However, because recent analyses have witnessed much cross-fertilization between perspectives (see Carruthers \& Smith 1996) the aim of this section is to highlight common stress points within these positions. This section briefly describes how the dominant theoretical perspectives are still highly controversial. There are sufficient critiques of each perspective for us not to dwell on individual theories. Instead, the focus will be on a fundamental flaw shared by all, thereby justifying the need for the alternative put forward in section 3 .

The phrase "theories of mind" signals the fact that the dominant theoretical position in research on mental state understanding has been that children construct theories of mental states which are similar to theories or paradigms in science. Change occurs either because children acquire a more sophisticated capability to represent mental state phenomena (Perner 1991), or because they modify their theories in light of evidence that is not compatible with their current framework of understanding (Gopnik 1993; Gopnik \& Wellman 1992; 1994; Wellman 1990). Theorytheorists claim that the child's understanding of mental states is theory-like because this knowledge is coherent and domain-specific, it shows a complex relation with the available evidence, postulates unobservable entities, and, most important, undergoes "paradigm shifts" when the child abandons one set of principles for another in the face of sufficient disconfirming evidence.

Stich and Nichols (1992) characterized a theory as a "body of rules or principles or propositions ... which serve to guide the execution of the capacity to be explained" (p. 35). Similarly, Gopnik and Wellman (1992; 1994) stated that a theory is a system of interrelated laws or rules that can be used to derive or infer predictions or explanations. In other words, understanding a particular action requires applying the theory's laws and deriving a solution. The editors of the special issue of Mind and Language (1992) in which two of these articles appeared suggested that such an approach is the most common strategy in cognitive science for explaining the ability to negotiate a particular psycho- 
logical domain. For example, this is the dominant approach in the area of language, but even here it has been severely criticized (e.g., Baker \& Hacker 1984; Tomasello 1995b).

Critics have long argued about whether the child's understanding can be equated with theoretical postulates within scientific movements (e.g., Feldman 1992; German \& Leslie 2000; Harris 1994a; Hobson 1991; Nelson et al. 1998; Scholl \& Leslie 2001). Russell (1992) argued that describing children's understanding of mind in terms of a series of theories does not provide an account of development. Gellatly (1997) claimed that drawing an analogy between cognitive development and historical change in theories conflates different levels of discourse and neglects the social dimension to child development and theory change in science. Campbell and Bickhard (1993, p. 33) suggested that if the term "theory" is used loosely it is general enough to include any form of human knowledge.

The view that children's understanding of mind is best explained in terms of one or more innate modules resulting in a "hard-wired" theory draws on arguments that the development of such social understanding is simply too important to be left to chance and that this understanding is acquired by children very early and without apparent effort or explicit teaching (e.g., Baron-Cohen 1995; German \& Leslie 2000). The position that an innately given module or set of modules is triggered at some point in development would seem to be the least consistent with developmental changes in children's understanding of mind (Gopnik \& Wellman 1994). It lives uneasily with the accumulating evidence, such as the sibling effect described above, showing that the nature of the social interaction children experience is closely related to the development of their social understanding. A further example is that secure attachment is associated with early false belief understanding (Fonagy et al. 1997; Meins 1997; Symons \& Clark 2000). This might be accounted for from the innate perspective by assuming that attachment depends on innate temperament, but this explanation is ruled out because the same infant can form different attachments with different adults (Meins et al. 1998). In addition, Tomasello (1999b) has argued that there has not been enough evolutionary time for the series of four mechanisms proposed by Baron-Cohen to have evolved. Further, Moore (1996) has pointed out difficulties with each of the sources of evidence (i.e., animal lesion studies, a study of neuroimaging, and studies of patients with brain damage) advanced by Baron-Cohen (1995) for the existence of modules or mechanisms. As in the debate over language development, we assume that there is agreement that children must be biologically prepared to develop an understanding of mind. The debate concerns whether the solution to this problem of understanding the mind is innately given or whether it is the capacity to develop such a solution that has evolved.

According to simulation theory, children develop an understanding of mental states through introspection, and use their imagination to reason about psychological matters (Harris 1991; 2000). We agree that imagination must play an important role in children's reasoning about the mind, but the introspective aspect of this approach is problematic for a number of reasons. Simulation theory faces the problem of how we can ever be confident that we can generalize our introspection to others (see Russell 1996, for a discussion). A more important difficulty for simulation theory is that the assumption that children learn the meanings of mental state terms through introspection is vulnerable to Wittgenstein's (1953/1968) private language argument (Chapman 1987a; Montgomery 1997; Russell 1996). Wittgenstein's argument - actually an anti-private language argument or arguments (Russell 1996) - seems to establish that children cannot learn the meanings of mental state terms just by introspection of their own inner experience.

In reviewing Wittgenstein's arguments we now move from the problems of the individual theories to a critique of a fundamental assumption shared by all theories discussed so far. The assumption in these theories is that the problem the child must overcome, through inference or introspection, is to figure out what is going on in the private and hidden realms of other minds. German and Leslie (2000, p. 230) set up the "fundamental problem of theory of mind" as follows: "[G]iven that beliefs, desires and pretends can be neither seen, heard nor felt, how does the young brain succeed in learning about them?" It is just this causal psychological view of the mind that Wittgenstein rejected. Wittgenstein (1953/1968) argued that our language "bewitches" people into thinking of psychological matters as inner objects, and his aim was to dispel misconceptions that our language leads one into. People assume that language refers to things, and in the case of beliefs, desires, and intentions we assume inner objects, or representations, that are causally related to behavior. We contend that this assumption is common to most theories of children's "theories of mind" and, that Wittgenstein's philosophy can be applied as a general critique of many current theories of social cognitive development (Racine 2004). Wittgenstein's goal, especially his private language argument, was therapeutic because his intent was to clear the ground of misconceptions, and we aim to use his remedy to propose a constructivist cure for the currently ailing approaches to mental state understanding.

Although there is general agreement about the importance of Wittgenstein's private language argument, there is considerable controversy regarding the meaning and implications, and even the location of the argument or arguments, in Wittgenstein's (1953/1968) Philosophical Investigations (cf. ter Hark 1990). ${ }^{4}$ By a "private language," Wittgenstein did not mean a new language that has not yet been taught to anyone else, or a dying language only spoken by one last speaker. Rather, a private language is radically private in the sense that it is not possible to teach it to others because it is based on private ostensive definition. Wittgenstein's rejection of the idea that one could define words privately is part of his critique of what he referred to as Augustine's view of language, according to which ostensive definition is the foundation for language because "it correlates words with things" (Hacker 1990, p. 99). Among the problems with this view of language, Wittgenstein (1953/1968) showed, is that "an ostensive definition can be variously interpreted in every case" (para. 28; emphasis in the original). An Augustinian might argue that the wordthing connection in language can be saved by the fact that one can "mentally point" - that is, when it comes to inner sensations we simply direct our attention. Thus, privately or mentally pointing would be unambiguous. However, this too would not work because it would require a person to "individuate a particular mental activity - sensation or impression - concentrate his attention on it, and label it" 
(Williams 1999, p. 19). Hacker (1996, p. 132) argued that there is nothing like mental pointing that could be similar to public pointing. One cannot concentrate one's attention inwardly and label a sensation. Thus, with regard to internal private states, nothing "could logically count as remembering correctly or incorrectly” (Hacker 1990, p. 108). This is just one aspect of Wittgenstein's general rejection of the whole view of beliefs, desires, and intentions as inner objects that one could introspect on and label.

Perhaps a more fundamental reason that words cannot be defined privately is that meaning is not attached to words, utterances, or representations in a mechanistic or dyadic manner (Goldberg 1991). Instead, meaning is based on use, on shared practices with others. Wittgenstein (1953/1968, para. 580) argued that "an 'inner process' stands in need of outward criteria."

According to Wittgenstein, the way to find out the meaning of psychological expressions is not to look inside the self, but to look at the function that the respective words and concepts play in our language. This is because the meaning of such expressions is determined by the ways in which they are typically used, not by the particular subjective impressions that we happen to have in these situations. (Chapman 1987a, p. 107)

It is important to emphasize that the Wittgensteinian view of beliefs is very different from that assumed by most work in the "theory of mind" tradition. In Racine's words (personal communication, November 2002),

when people in the field speak of beliefs as mental contents, they reify belief into a new form of mental content that is independent of activity but yet causes activity. There are no such contents. Belief exists and is created in action, not in the head. We should not take the development of an ability to re-present activities off line to be the development of an ability to experience inner states of belief. (see also Racine 2004)

It is this Wittgensteinian view of beliefs and intentions that we assume in the alternative theory we propose in this target article.

We should be clear that Wittgenstein was arguing against the idea that introspection could be the way one learns the meaning of psychological terms. But he did not rule out introspection in some cases once someone has learned to talk about the psychological world, such as in the following example: "Does it make sense to ask: 'Do I really love her, or am I only pretending to myself?' and the process of introspection is the calling up of memories; of imagined possible situations, and of the feelings that one would have if . . ." (Wittgenstein 1953/1968, para. 587; see also Hacker 1996, p. 133). We should also emphasize that Wittgenstein was not denying that one experiences sensations privately. This was not the target of his private language argument. Rather, he rejected the idea that it would be possible to learn the meaning of psychological words through introspection.

We have pointed out some difficulties with the dominant theoretical accounts of children's mentalistic understanding (for further criticism see, e.g., Gellatly 1997; Hobson 1991; Nelson et al. 1998; Russell 1996). There are, however, different versions of these positions and we lack the space to do justice to the complexity of these theoretical approaches. We also recognize that criticisms may be countered with auxiliary hypotheses. These theoretical positions converge on some points. That is, most theorists would agree that infants must be innately prepared to learn about the social world, that their own inner experience must play some role in the development of this understanding, that such knowledge is interconnected and changes with development, and that imagination is important in the process of social reasoning. Within such general agreement, however, there is still much controversy, and there is an urgent need for an alternative theoretical account that is more consistent with the accumulating evidence of the important role of social interaction in the development of children's social understanding.

To summarize our argument thus far, a common problem with the dominant perspectives in the field is that each focuses on the cognitive architecture of mental state reasoning, without reflecting on the social landscape in which such reasoning is constructed (Astington 1996; Lewis et al. 1996). It is not by coincidence that recent discussion has begun to consider this social dimension as more central to development (Astington \& Baird, in press). Astington's (1996) Vygotskian approach more explicitly includes such a social dimension. However, as we discussed in section 2.1, we must carefully distinguish social constructionist approaches that are equivalent to passive enculturation from social constructivist positions that also recognize the social dimension as essential but claim that passive adoption of cultural norms cannot account for all development. So far, Astington (1996) has not been explicit on this issue, and the resulting ambiguity tends to lead to the enculturation interpretation. Although Vygotsky's approach could be read as an enculturation theory, more complex readings are possible and more plausible (e.g., Fernyhough 1996). In section 3 we construct an account that is in keeping with such a reading.

\section{A solution: Constructing an understanding of mind}

In the preceding sections we contrasted approaches based on the assumption that the development of an understanding of mind is an individual process of introspection, maturation, or the formation of a theory, with the contrasting position that children acquire culture-specific concepts regarding the mind which are passed on from the social group. Instead of choosing between these two contrasting positions, we begin from a different starting point and endorse an alternative perspective emphasizing the relations between people (Piaget 1977/1995). Concepts about the mind are not just passed on from the social group, nor are they completely formed by individual child-theorists. Instead, children gradually construct social understanding through the regularities they experience in interacting with others.

Our approach to the development of children's social understanding is based on Chapman's (1991; 1999) reformulation of Piagetian theory emphasizing the social dimension in development. Piaget is now known for his emphasis on subject-object interaction, even though in his early work (e.g., Piaget 1924/1928), and continuing throughout his career (Piaget 1977/1995) he was also concerned with the social dimension of development (Chapman 1988a; Lourenço \& Machado 1996). Vygotsky (1978; 1934/1986), on the other hand, is known for his concern with the role of social interaction in the development of higher mental functions. An integration of subject-object interaction and social interaction results in a triangle "consisting of an active subject, the object of knowledge, and a (real or implicit) inter- 
Carpendale \& Lewis: Constructing an understanding of mind

locutor, together with their mutual relations," which Chapman (1991, p. 211) termed the "epistemic triangle." Chapman argued that "although Piaget recognized the importance of both operative and communicative forms of interaction in various phases of his work, he never integrated those components in a single model" (p. 212). This epistemic, or "knowing," triangle preserves the insights of Piaget and Vygotsky and facilitates thinking about how social interaction and subject-object experience can be reconciled in development. The epistemic triangle has much in common with Hobson's (1994; 2002) notion of a "relatedness triangle," except that the latter focuses almost exclusively on affective engagement, particularly in infancy.

Chapman employed the notion of the epistemic triangle to describe the role of social interaction in the development of knowledge in the area of children's reasoning on concrete operational tasks (Carpendale 1999a; Carpendale et al. 1996; Chapman \& Lindenberger 1992). We take it to be the lowest common denominator for understanding development in general and it is equally applicable to children's reasoning regarding social matters. The fundamental point is that social interaction is essential in the development of cognitive, social, and moral knowledge. Children "construct a new conception of reality by coordinating their object-oriented operative interactions with those of other people, by means of their communicative interactions with the individuals in question" (Chapman 1999, p. 34; emphasis in the original). At the same time that children are constructing knowledge of the physical world they are also constructing knowledge of other people. It is through communicative interaction that children discover that others sometimes have different beliefs about the world. We take it that children assume a stable external world that remains the same for themselves and other people. In order to maintain this assumption when they encounter others who may have different beliefs about aspects of the world, such as the location or identity of objects, children change their expectations or their understanding of mind and of how beliefs are formed. At some point children realize that access to information, for example through seeing, is essential in the formation of beliefs. That is, children construct an understanding of how they and other people acquire knowledge of the world and they may achieve comparable levels of development at similar ages because of commonalities in their experience.

From this perspective, knowledge is gradually constructed within social interaction. Children's social knowledge is based on action; it is not theoretical in the sense of a set of laws formed on the basis of observation to explain the doings of other people. Understanding others is at first practical. It is gradually constructed through regularities in interaction with others. At first the interaction between infant and other (e.g., the caregiver or sibling) is dyadic and not yet referential, but by the latter part of the first year this interaction becomes triadic, between the infant, the caregiver, and objects. ${ }^{5}$ That is, aspects of the world become increasingly included in the interaction between infant and caregiver. The infant is embedded in this triadic interaction but only gradually differentiates the self, other people, and the world of objects. In keeping with this gradual view of development, we expect to see children exhibit early forms of knowledge about the mind first in interaction; only later will children become able to reflect on such knowledge.

In the following subsections we flesh out this view of de- velopment in more detail, first briefly in development during infancy and next when children begin to learn words.

\subsection{The development of the epistemic triangle: Social understanding in infancy}

Triadic interaction between the child, another person, and the world is essential in the development of knowledge in general, and here we focus on social knowledge. This leads to the question of how and when the capacity for triadic interaction develops from an infant's dyadic interaction with either a parent or the world of objects. There is much debate and discussion concerning how and when infants achieve the capacity for triadic interaction - or move from "primary intersubjectivity" to "secondary intersubjectivity" (Trevarthen 1979; Trevarthen \& Hubley 1978). ${ }^{6}$ Examples of behavior involving triadic interaction begin toward the end of the first year of life and include social referencing, gaze following, and different forms of pointing. One of the early manifestations of triadic interaction between the infant, an adult, and objects during infancy occurs at about 12 months of age when infants start to point to objects (Schaffer 1977). Bates et al. (1976) distinguished between infants' use of pointing gestures in order to make requests ("protoimperatives") and pointing to direct adults' attention to objects ("proto-declaratives"). At this age, infants appear to be actively directing an adult's attention. These are two forms of behavior that at least appear to involve joint visual attention. This capacity for joint attention plays an essential role in word learning during the second year (e.g., Baldwin 1995; Lock 1980). The triangle between the infant, the parent, and the world in action can be observed even earlier, at about 9 months of age, in the phenomenon of social referencing, in which an infant apparently refers to a parent's facial expression to determine their evaluation of an ambiguous situation (e.g., Walden \& Ogan 1988).

Any claim about the age at which joint attention behaviors such as pointing, gaze following, and social referencing develop will be controversial. Different forms of these behaviors develop at different ages and, because there is much debate about what these behaviors may reveal concerning infants' social understanding, both rich and lean interpretations of this apparent evidence of early social understanding in infancy are possible (Baldwin \& Moses 1996; Moore \& Corkum 1994). Richly interpreted, those behaviors involving joint visual attention seem to be evidence of infants' understanding that other people have intentions and attention that can be directed to various aspects of the world (Carpenter et al. 1998; Tomasello 1995a). The behaviors mentioned above have been interpreted as evidence that infants at this age already have an "implicit" "theory of mind" (Bretherton 1991; Bretherton et al. 1981). From the perspective of this rich interpretation, infants require a mentalistic understanding of attention in order to engage in joint attention with adults and, therefore, this behavior reveals such an insight.

Theorists taking a rich view of joint attention can be seen as falling within an individualist framework. According to that framework, social development begins with the first person perspective and the individual's own self-knowledge which then must be extended to others; that is, "knowledge of other minds is parasitic on our knowledge of our own mind" (Jopling 1993, p. 291). With this starting point for self-knowledge, the route to an explanation of understand- 
ing other minds is usually through the analogical argument. This argument has an extensive history. A current use of it by Meltzoff et al. (1999) is the following: "(a) When I perform that bodily act I have such and such a phenomenal experience, (b) I recognize that others perform the same type of bodily act as me, (c) the other is sharing my behavioral state; ergo, perhaps the other is having the same phenomenal experience" (p. 35). Tomasello (1999b, pp. 70-76; 1999a, p. 68) also relied on the analogical argument in his theorizing about infancy (although his theorizing concerning language is consistent with ours).

The analogical argument relied on by the individualist framework is problematic for a number of reasons (e.g., ter Hark 1990). For example, it seems to attribute too much to the infant in counterfactual reasoning and the ability to take an external perspective on their own experience (Soffer 1999). Analogical reasoning is possible once children have the concept of self and other, but the argument already presupposes this distinction and on that ground cannot logically be its source (Scheler 1954, pp. 240-41). (For further criticism of the analogical argument see Müller \& Carpendale 2004.)

More recent accounts of joint attention support a leaner interpretation. For example, Baldwin and Moses (1996) explored development in triadic interaction in the context of social referencing and found that infants look at their mothers under conditions of stress but not to gather information. This suggests that triadic interaction may be important in further development of infants' social understanding, but at $9-12$ months of age it is only very rudimentary and is more likely to be a manifestation of the attachment system than social understanding.

The most well-known lean account is that of Moore and Corkum (1994; Corkum \& Moore 1995; 1998), who showed that infants engage in joint visual attention, or end up in situations in which their attention is coordinated with adults, through processes such as conditioning, without having yet developed an understanding of attention (for a similar argument, see Kaye 1982). Rather than joint attention necessarily revealing infants' understanding of other people's attention, Moore and Corkum (1994) suggested that there are developmental changes in infants' understanding of joint visual attention. They provided evidence that around the end of the first year infants rely primarily on observing the adult's head turn without apparently understanding the importance of eye direction. This appears to call into question the commonsense view that infants of this age understand that the other person is paying attention to the object. Later, during the first half of the second year, infants begin to use eye direction information as well as the head turn to achieve joint visual attention. This suggests that experience in which infants' attention is coordinated with adults' attention occurs before infants understand much about attention (Moore 1999). The infant becomes embedded in interaction with some success and only through the experience of that interaction later develops a more complete understanding:

Once the infant is reliably engaging in joint attention, the experiences offered by this new triadic interactive form of behavior will afford progress in the infant's conceptualization of attention and set the child on the road to the discovery of all forms of social life that rest on sharing attention with others. (Corkum \& Moore 1995, pp. 81-82)

This position is also supported by research on infants' pointing (following Murphy \& Messer 1977). Desrochers et al. (1995) reported that in a longitudinal study the majority of infants began to use noncommunicative pointing (i.e., pointing without looking at their mother) at 12 months of age. However, only beginning at 15 months did these same infants begin to use pointing that seemed communicative in intent - for example, by simultaneously looking at their mothers to ensure they were successfully communicating. Desrochers et al. documented the development of increasingly sophisticated forms of pointing between the ages of 12 months and 18 months. Here again the evidence suggests that infants engage in action such as pointing, which may result in achieving joint visual attention or the coordination of attention with an adult, because the parent may attend to the object to which the infant is pointing. However, these young infants do not as yet appear to understand what they are doing because they do not look at the parent's face. In further exploring the development of pointing, Moore and D'Entremont (2001) found that infants at age 1 year are more likely to point when their parent is looking at them, whereas at age 2 years they tend to point when their parent had not seen an interesting sight. This suggests that young infants point to enhance interaction, but older infants understand pointing as directing others' attention.

Another reason for caution concerning the acceptance of overly rich interpretations of the meaning of early joint attention comes from research with nonhuman primates. Chimpanzees can follow gaze, and on the surface their behavior looks surprisingly similar to that of infants, but Povinelli (1999) showed through a series of experiments that chimpanzees do not appear to understand the psychological significance of gaze direction. For example, chimpanzees trained to beg with a hand-out gesture and given the opportunity to beg for food from one of two experimenters sitting side-by-side are initially just as likely to beg from someone with her eyes closed, with her head turned away, or even with her head covered by a bucket as they are to beg from someone with her eyes open. It takes many trials for the chimpanzee to learn to beg from the person who can actually see their begging gesture (Povinelli 1999).

Proponents of the lean interpretation of joint attention have much in common with our position on infant social understanding. In particular, Moore (1999) set the origins of infant social understanding within triadic interaction (equivalent to subject, interlocutor, and object):

One might say that the 12-month-old recognizes intentional relations but only to the extent that she can share such a relation with an interactive partner. For the 1-year-old, intentional relations exist in the interaction and are not a property of, or descriptive of, individuals. (Moore 1999, p. 48)

However, Barresi and Moore's (1996) more detailed account starts from an individualistic perspective in which infants have different information about self and other and the integration of these two sources of information leads to the development of an understanding of self-other equivalence:

An individual organism's information about intentional relations is available from two different sources. An organism has direct first person information about its own intentional relations and direct third person information about the intentional relations of other agents. (Barresi \& Moore 1996, p. 108)

This implies that a self-other distinction is already in place, whereas from our perspective it is this distinction that requires an explanation.

An alternative approach that avoids these problems 
within an individualist framework is the relational framework (Jopling 1993), according to which the development of social understanding begins from relations between people rather than from individual self-knowledge. The starting point in development is a position of relative nondifferentiation between self, other, and the world (Baldwin 1906; Hobson 1993; Merleau-Ponty 1964; Piaget 1936/1963, 1937/1971; Vygotsky 1998; Werner \& Kaplan 1963). From an observer's point of view one sees the infant interacting with others and the world, but at first the infant has not constructed these distinctions. The differentiations between inner and outer, subject and object, and self and other occur gradually within interaction (Müller \& Runions 2003). The process by which the infant shifts from participating in triadic interactions to an understanding of the distinction between her own and others' intentions remains a holy grail within this area of theorization. However, only the constructivist perspective starts from a point where this problem is not already "solved" - as stated above, accounts within the "theory of mind" tradition assume self-other differentiation from the outset.

A central assumption from a constructivist perspective is that knowledge originates in activity. Infants interact dyadically with the world of objects as well as with people, and through this activity they develop sensorimotor action schemes. Such schemes embody knowledge because they are modified as a function of the differences between what is anticipated and what the infant actually experiences (Chapman 1999). With further development, two or more action schemes may be combined, resulting in more objectivity or separateness from the infant's own action. The infant develops expectations about what can be done with objects as well as expectations about their interactions and routines with people. And infants begin to regard people as independent "centers of causality" (Piaget 1937/1971); that is, people do not always do what the infant expects or wants (Baldwin 1906). Different patterns of activity are possible with people as compared to with objects. There has been a great deal of research attention devoted to the study of faceto-face dyadic interaction between infant and parent. In such interaction infant and parent respond to each other and this could be described as a form of communication but it does not yet refer to anything outside of this dyadic interaction (Chapman 1991). Objects may begin to be included in this interaction in routines such as giving and taking. From this perspective, infants first experience triadic interaction that is supported by the parent or through processes such as infants learning to associate head turns with interesting sights (Corkum \& Moore 1998). At this point infants still do not have a clear understanding of others' attention; but over time they will construct such an understanding through interaction (Müller \& Runions 2003). Development involves a process of gradually constructing action schemes and combining them. Infants slowly differentiate and coordinate their attentional directedness toward objects with others' directedness toward objects (Müller \& Carpendale 2004).

Hobson's (2002) relatedness triangle is a similar approach that emphasizes the importance of the infant's emotional engagement. To this important insight, we add that the infant's understanding of causality and spatial relations is also critical in the infant's ability to engage in joint attention. This point is reflected in the various forms of gaze-following that are observable from about age 3 months to age
18 months. More complex forms of this social behavior, such as following an adult's gaze to an object behind the infant, require the infant to have an understanding of herself as an object within a world of other objects (Müller \& Carpendale 2004).

When infants have developed the capacity to coordinate attention with others they can refer to aspects of the world by directing others' attention, and they can understand other people's referential intent. Reference involves more than just forming an association between a sign, usually verbal, and an action, as is the case in the few triadic chimpanzee gestures (Tomasello \& Camaioni 1997). Instead, signs can be used flexibly to convey different meaning in different contexts, and this requires the ability to infer others' referential intent. ${ }^{7}$ Recent "theory of mind" research on infancy has worked with inventive experimental procedures, but neglects an older tradition (e.g., Lock 1978; Schaffer 1984b) that attempted to chart the development of such signs within the richness of the infant's daily interaction rituals and experiences. Our position is in keeping with this tradition and the more recent view of Reddy (1991) that the rituals preverbal children engage in during "teasing" and "mucking about" show the beginnings of the process by which children come to share attention and to construct a practical or lived form of social understanding or knowledge of other people.

The ability to achieve joint attention and to understand other people's referential intent is the foundational insight on which language is based (e.g., Baldwin 1995; Sinha 1999). Tomasello (1999b; Tomasello et al. 1993) argued that it is this uniquely human ability that allows people to make use of culture to a far greater extent than can other animals. This ability is based on an initial level of social understanding that gives infants the capacity to engage in triadic interaction, and such interaction then provides a context for further social cognitive development. Infants now start to use actions and words to refer to other things. Their communication at this point becomes referential; that is, infants indicate what they want the adult to attend to and also are able to determine adults' referential intent. At this point, "words begin to mediate children's sensorimotor acquaintance with reality. Children no longer know reality solely in terms of what they could do with it, but also in terms of what they could potentially say (or hear) about it" (Chapman 1999, p. 34; emphasis in the original). All of the components of the epistemic triangle are now in place. In the next section we discuss how this new level of knowing provides a context for further development in children's understanding of the social world.

\subsection{Outer criteria for inner processes: Talking about the psychological world}

As infants develop the capacity to coordinate attention with others, they begin to learn language. Children's word use is built onto the shared practices they have already developed, such as following or directing another's attention, making requests, and conveying their intentions or goals. Learning to talk is grafted onto prior ways of acting and interacting with others. This is a view of language as activity, not just as information exchange. In this section we are concerned with the development of children's ability to talk about the psychological world. This raises the issue of the relations between language and thinking. A common assumption is 
that concepts are acquired first and then the words for such concepts. In contrast, from a Wittgensteinian perspective, language and thinking are much more tightly intertwined. Wittgenstein (1981a, para. 324) asked, "Does a child learn only to talk, or also to think? Does it learn the sense of multiplication before or after it learns multiplication?" We assume that the child learns about the concepts and the words at the same time. That is, learning the meaning of words to do with multiplication involves learning the operations that are criterial for those words.

The research on children's language and their social understanding initially used language as a window on children's understanding and tended to focus on words that seem most obviously to be mental state terms such as "think" and "know." Other words, however, that are not obviously mental state terms at all (e.g., "hide") involve some understanding of how people know things and of how people can be prevented from knowing things (Turnbull \& Carpendale 1999a; for similar discussion see Russell 1992). In fact, it would be difficult to draw a clear line between words that do or do not turn on some understanding of the psychological world. Hacker (1991) pointed out that saying a robot or an automatic door opener "sees" something is parasitic on the primary use of such a verb. With reference to human agents, simple words such as "look" and "see" seem to refer to perception, but their correct use is tied to an understanding of the perceiver's psychological attributes when they attend to something. Learning the use of such words is rooted in children's everyday experiences of coordinating attention with others. Children can be asked the false belief test question by referring only to where Maxi would look (the "look question") without use of the word "think." In fact, in some research young 3-year-olds seem to pass this form of the test question before the "think" version of the question (Chandler \& Hala 1994; Hala \& Chandler 1996). Similarly, 3-year-olds pass a false belief question phrased with "say" before a similar question phrased with "think" (Nelson et al. 2003). The general point is that a great deal of talk that is based on an understanding of knowledge acquisition and the mental world may not, in fact, involve what we would usually consider to be mental state terms (Turnbull \& Carpendale 1999a).

In describing how children learn to talk about the psychological world it is necessary to draw on Wittgenstein's notion of criteria. According to Chapman's operational semantic theory (Chapman 1999; Carpendale et al. 1996), children learn about the meaning of relational words such as "longer" through learning the operations that are criterial for the use of such words. Children may come to understand that the meaning of "longer" in the case of length involves the operation of checking to see which of two sticks protrudes farther. Only through elaboration do children learn the additional criterion of first lining up the ends of the sticks. Terms like "longer" are also involved in other language games referring to time. In the present case of learning how to talk about the psychological world the criteria for the use of such words are embedded within social interaction. As discussed above, Wittgenstein's (1953/1968) private language argument stressed that it is not possible to learn through introspection the meanings of words referring to inner processes (Chapman 1987a; Montgomery 1997; Russell 1996). It follows from this that children learn about inner processes (their own and other people's) through public criteria, not only through their subjective experience; that is, they learn the appropriate contexts for the use of various words referring to the psychological world. Children learn the pattern of interaction for which it is appropriate to use a particular term, for example, mental or emotional, or dealing with pain, and so forth. Wittgenstein's (1953/1968) view of how children learn the meaning of sensation terms is that

words are connected with the primitive, the natural, expressions of the sensation and used in their place. A child has hurt himself and he cries; and then adults talk to him and teach him exclamations and, later, sentences. They teach the child new pain-behavior. (para. 244)

Wittgenstein's argument regarding sensation words also applies to psychological words (Chapman 1987a). Children learn to talk about the psychological world in a variety of ways including discussions about themselves and other people. That children appear to develop the ability to understand another's belief at the same time as their own (Gopnik 1993) would suggest that they learn the criteria for each at about the same time. Criteria for talk about the mental world are behavioral evidence for inner experiences such as sensations, emotions, thinking, remembering, imagining, and so forth: "Criteria are those publicly observable circumstances which might be used in teaching the correct use of the expression to a child or someone else learning our language" (Chapman 1987a, p. 105).

From a Wittgensteinian perspective, the use of psychological terms becomes part of earlier, or more "primitive," prelinguistic behavior (Hacker 1997). Words such as "see" and "look" may be grafted onto earlier joint attention behaviors. These words can then be used as new ways to direct and follow attention. Similarly, emotion words may be grafted onto the child's prior reactions or behaviors. The circumstances surrounding the use of psychological words become the criteria for their use. Talk about the mental world is built onto prior activities as a refinement (Canfield 1993; 1999; Malcolm 1991; Turnbull \& Carpendale 2001).

It might seem that if we endorse the Wittgensteinian idea that children learn about the mental world through learning how to express in language their feelings, plans, goals, and so forth and through learning the criteria for the third person use of various psychological terms, then we must endorse an enculturation position in which mentalistic concepts are imported from the social world to the individual. As mentioned in section 2.1, this is one interpretation of Wittgenstein and it implies a cultural relativism by which children would just learn the mental concepts used in their particular culture. However, we do not endorse this interpretation for two reasons. First, at the basic level of social understanding (e.g., seeing, looking, intentions, desires, and beliefs), children's understanding is built onto shared practices that we expect would be common across cultures because these are common aspects of human experience (for a parallel argument within the theory view, see Wellman 1998). This does not rule out that there may be crosscultural variability, such as in complex emotions (Lillard 1998).

Second, and central to our argument, children's development is constructive. This involves combining the epistemic triangle approach to development with Wittgenstein's concept of criteria. That is, children do not acquire an understanding of talk about the psychological world in an all-or-nothing fashion. Concepts are not passed on, ready-made, through language. Criteria are multiple and 
children may initially acquire a subset, which enables them to use words apparently correctly in supportive contexts. It is only through communicative interaction with others about beliefs (sometimes differing, sometimes concordant) about the world, that children gradually construct an understanding of belief. In other words, although young children often use and seem to understand words based on an understanding of the process of knowledge acquisition (e.g., "see," "remember," and "guess"), their understanding is as yet incomplete and is based on a partial set of the criteria for the correct use of such words (Montgomery 1997). For example, Lillard (1993b) showed that when young children first begin to use the word "pretend" they focus on the more obvious criterion of the action being performed rather than on the individual's knowledge or intentions. This view is consistent with the generally protracted nature of language development (e.g., Nelson 1997).

We now turn to a consideration of other accounts of the relation between language and social understanding. The finding that various aspects of children's linguistic ability are associated with false belief performance has already been mentioned (e.g., Cutting \& Dunn 1999; de Villiers 2000; de Villiers \& de Villiers 2000; Happé 1995; Jenkins \& Astington 1996). One approach is that language can be used as a window to reveal the child's social understanding. Bartsch and Wellman's (1995; see also Sabbagh \& Callanan 1998) work exemplifies the usefulness of such research in that it shows that children produce terms referring to desires before they refer to beliefs. However, one should be careful of the assumption that mental terms refer to mental states. Even the simple word "want," which would be coded as a desire term, can be used in many ways, such as to make requests or to make offers. Budwig (2002) found that mothers and their children used the word "want" in different ways. Regarding syntax, the de Villiers studies (e.g., de Villiers \& de Villiers 2000) showed that the child's grasp of the syntax of complementation (i.e., the fact that mental state verbs necessarily take complementary clauses when they refer to mental states, like other non-mental state verbs; e.g., He thinks that he had eggs for breakfast; He said that he had eggs for breakfast) is correlated with false belief test performance. Such analyses suggest important connections between mental state understanding and the ability to parse statements about mental states. Longitudinal evidence indicates that language competence, as measured in standardized tests, appears to predate the development of false belief understanding (Astington \& Jenkins 1999).

Each piece of research shows how children's understanding of mental states is likely to be related to their general proficiency in understanding and producing words and sentences. However, such correlations raise the issue of causal direction, third factors, and the nature of any influence. Indeed, they are somewhat antithetical to our approach because language and social understanding are so intertwined that it is somewhat artificial to separate human activity into parts and call one part language and the other social understanding. This position follows, in part, from viewing language as activity (Turnbull 2003; Turnbull \& Carpendale 1999a). However, if we do talk in terms of language and social understanding, then, from the perspective of the epistemic triangle, language is important for two reasons. Astington (2001, p. 686) has described these two roles, and the need for a resolution, as "a means for representing false belief in contradistinction to the evidence given in re- ality and it is also the means by which children become aware of beliefs, both content and attitude.... What is needed is a new conceptualization that reconciles and combines insights from both views." Although we may differ from Astington in what we mean by representation - from our perspective, language mediates children's knowledge of reality - children learn the criteria for words to talk about human activity and then can reflect on the psychological world. Language, or communicative interaction, is the means through which children learn about other people's experience and so develop a more complete set of criteria.

Parallel arguments have been made within more traditional accounts. Harris (1996), in particular, had the insight that conversation is important. Rather than mere exposure to mental state terms, Harris argued that conversation is important because it is a constant reminder that other people have different perspectives. We agree that this is one role conversation plays, but we contend that this is not sufficient. Communicative interaction helps children realize when they only have a partial set of the criteria for using words such as "look," "see," "think," and "know" correctly, and when their understanding of the patterns of interaction in which these words are used is incomplete. Conversation has a pivotal role within the epistemic triangle, but as part of an integrated system not as an independent factor. To maintain the assumption that one lives in a common, stable, external world that is the same for the self and others - one of the basic presuppositions required in any attempt at communication - children must develop an increasingly sophisticated understanding of the nature of the mind. Maintaining the assumption of an independent external world in the face of evidence of other people's differing experience of the world requires that children modify their understanding of the relations between their own and others' beliefs about the world to include the notion that one's beliefs about the world depend on the information to which one is exposed.

Once children can talk about the social, emotional, and psychological world, they can begin to reflect on or think about people's activity in psychological terms (Chapman 1991; Piaget 1945/1962; Vygotsky 1934/1986). This raises the issue of how we view the process of reasoning. In the theories we reviewed earlier in this target article, the process of reasoning involves simulation or the application of rules that are either innate or formulated by the child on the basis of observation. In contrast, we suggest that children understand talk about the psychological world in terms of the patterns of activity that are criterial for the use of such mental state terms - that is, the pattern of interaction for which we use these words. Reasoning, then, involves the coordination of these activities. Thus, as well as understanding questions in terms of human interaction, children also require the ability to imagine and coordinate activities that may not be immediately present. From this perspective, reasoning is not based on the application of rules, but particular instances of rules would be manifest in the process of reasoning (Carpendale et al. 1996).

\section{Using the epistemic triangle to explain the "core findings" in "theory of mind" and the influence of relationships}

In this section we address the "So what?" questions that necessarily follow in response to the proposed position in sec- 
tion 3. In section 4.1 we take the paradigm that appears to show most clearly that a clear conceptual shift takes place - the false belief test - and compare a traditional theorytheory account with the one offered here. In section 4.2 we explore whether the theory presented here can go further than previous ones in accounting for the correlations between a range of social relationships, like the sibling effect, and mental state understanding. We suggest that it can do so more convincingly than any other account.

\subsection{The gradual acquisition of criteria within conversation}

We are not the first to criticize the dominant accounts of "theory of mind" development by suggesting that such skills are acquired gradually. Such gradualism has been explained within a framework of other arguments, like the protracted development of language (Nelson 1997), and from within a philosophical critique of the theory-theory approach (Woodfield 1996; see also Russell 1996). It is therefore important to outline how gradualism follows from our theoretical perspective. Theory-theorists might depict a series of theories, "rudimentary prototheories" (Ruffman 2000, p. 263) like "preliefs" (Perner et al. 1994), or processing demands in different procedures. This account can go only so far. However, an argument based on gradualism cannot be an excuse for fuzzy thinking. It needs a theoretical foundation and it needs to provide an explanation of the data.

The notion of gradualism underlies the type of developmental approach taken by Piaget, and also Vygotsky. For example, as Chandler (2001) has pointed out, in Piaget and Inhelder's (1948/1967) research on the development of children's understanding of visual perspective-taking they described a sequence of different forms of understanding. This developmental approach has often been lost in the subsequent role-taking literature and the current "theory of mind" research. The strong tendency has been to collapse this protracted development into a single transition point (Chandler 2001; Chandler \& Carpendale 1998).

The view that an understanding of mind unfolds gradually may appear to be a nonstarter when the collected data on the false belief test are examined. The recent metaanalysis conducted by Wellman et al. (2001) attempted to identify the factors that reliably facilitate performance in the false belief test. In an analysis of 178 experiments with 591 conditions, Wellman and colleagues found that factors like the type of task used, or whether the target question focused on the protagonist's thoughts or his or her actions, were stable across studies. There seemed to be a clear conceptual shift in children's performance at around age 4 , and this is consistent with the theory-theory account of developmental change. However, consistent patterns of performance on one procedure do not necessarily inform us about the nature of change - from our theoretical perspective they may simply show that four-year-old children across the many studies engage in sufficient interaction with others about the veracity of beliefs to pass this test. As Scholl and Leslie (2001) noted, the meta-analysis might only show the sorts of improvement with age seen on most developmental tasks.

Indeed, proponents of the theory-theory perspective have long warned us about the problem of "neurotic task fixation" (Gopnik et al. 1994, p. 157), and there are dangers in relying on only one task, particularly those versions of that task which do not test the variations in performance. In Wellman et al.'s (2001) meta-analysis some types of studies were not considered. Moses (2001) pointed out that among the types of studies not included are studies involving explanations of false beliefs, deceptive behavior, and eye movements rather than verbal responses. Even within the homogeneity of tests that were likely to lead to homogenous results, there was still considerable variation among samples, and the authors found that

several task manipulations do increase young children's performance: framing the task in terms of explicit deception or trickery, involving the child in actively making the key transformations, and high-lighting the salience of the protagonist's mental state or reducing the salience of the contrasting real-world state of affairs, all help young children to perform better. (Wellman et al. 2001, p. 672)

Two issues emerge from Wellman et al.'s interpretation of the collected false belief data, which are typical of the "theory-theory" approach to the literature: the extent of task variation and its implications.

In contrast to the theory-theory, the constructivist view actually predicts that there will be variations in the child's acquisition of knowledge within a domain. For example, when exploring the development of children's social understanding, Selman et al. (1983) found different levels of perspective taking when the child was involved in an activity than when the child was reflecting on it. The same analysis can be applied to the types of data reported by Wellman et al. (2001) and the findings, listed above, which they chose to omit from their analysis. For example, Wellman et al. found that if the protagonist's motive is made explicit or if the child actively participates in the procedure (e.g., Chandler \& Hala 1994; Hala \& Chandler 1996) then preschoolers pass the test in greater numbers. Similar results are found when child participants act out the answer by demonstrating with a doll (Freeman et al. 1991). We propose that children do better on false belief tasks when they are actively involved, because their understanding of the events is supported by the social interaction. Not only is their attention directed by the experimenter (within the epistemic triangle, the subject-interlocutor interaction), but also their involvement in important aspects of the events commits them to acting on the object of knowledge (the subject-object of knowledge part of the triangle). Thus, children's thinking is supported by their practical activity within a dynamic triadic process. With further development in their ability to hold different aspects of events in mind and coordinate them, children will be able to deal with false belief situations at a more abstract level. This type of research provides a snapshot of mental state understanding in the making. It reveals a microcosm of relationships, which current studies are showing to be vital and which explain patterns of findings to be revisited in section 4.2.

A constructivist perspective would predict that the performance of children who are within the process of developing these insights would be influenced by many aspects of social interaction. Such gradualism is highlighted by recent research showing that there are other influences of the context of the assessment procedure. For example, there is inconsistency between laboratory-based assessment procedures - standard false belief tests - and naturalistic observation of young children. Children typically pass standard false belief tests only by the age of approximately 4 years, whereas in the home parents report observing their $2 \frac{1}{2}$ or 
young 3-year-olds apparently demonstrating an understanding of false belief (Astington 2000; Newton et al. 2000). In a diary study, Newton et al. (2000) found that parents reported many incidents in which their young children were involved in deception even though these children were known to have failed false belief tasks. In agreement with our position, Newton et al. suggested that rather than early deception-reflecting insight, young children engage in deceptive acts with only partial understanding and such experience is the context for learning about deception.

Wellman et al. (2001) claimed that although factors such as the child's active involvement improved performance on false belief tasks, this evidence "failed to fit an early competence model" (p. 674) because those factors "do not raise the youngest children's performance to systematically above-chance performance" (p. 674) and there were no interactions between these task manipulations and age. Wellman et al. derived "the essential claim that such task factors mask early competence" (p. 672). The difficulty with this interpretation is that Wellman et al. appear to have interpreted arguments for gradualism within their own way of thinking about competence, resulting in the straw person they term the "early competence model." Wellman et al.'s inference is based on a prediction that the child either has or does not have false belief understanding, which may be hidden by performance factors. This is based on the assumption that competence is a hidden underlying factor that causes performance (Chandler 1991). However, there are sufficient data showing how children are able to understand false beliefs at different times to support the claim presented here that we need to explore the process of change in greater detail. The general problem is that if an individual has developed a particular competence, why does he demonstrate it in some situations but not in others? Is this variability measurement error or evidence of gradual development? ? $^{\text {Is }}$

Variability in performance across different tests is usually explained in terms of performance factors masking the child's competence. But, as Chapman (1987a) pointed out, it is not always possible to separate performance factors clearly from the underlying competence. The assumption that there is one competence that is presumed to arrive all in one piece, like a theory or a set of rules, is what Chapman (1987a) referred to as the "measurement model." As an alternative approach he proposed the "membership model," according to which there are varying degrees of understanding that are assessed with different procedures. Here we could say that degrees of membership, or competence with mental state concepts, are related to children's increasing grasp of the multiple criteria for words referring to the mental world and their ability to understand events outside of supported social contexts in their immediate practical activity.

The perspective we apply draws on Vygotskian (1978; 1934/1986) ideas according to which children's initial, fragile social understanding, or "understanding-in-action" (Dunn 1996a), is at first evident when supported by social interaction. Instead of naturalistic observation of early competence (e.g., Newton et al. 2000) being the application of a theory, this type of experience is necessary to gradually construct social understanding. This claim that children begin talking about the mental world with only a partial understanding is consistent with work on language learning in general (Nelson 1997). This is not, however, a simple adop- tion of culturally available concepts concerning the mental world; rather, children must come to understand these concepts through becoming competent in using such words in their practical activity. Children's correct use of psychological words is tied to their understanding of social situations and human activity. This understanding is facilitated by relationships that help the child understand other points of view. With experience in such social interaction concerning the mental world, children's understanding becomes progressively consolidated. That is, children are able to think about situations abstracted from their practical context of interaction and are able to anticipate and reason about situations such as the false belief task. The ability to talk about the mental world gives children a resource with which to reflect on and understand others as well as themselves.

The fixation on false belief understanding has prevented us from examining the longer view of development. As Chandler (1988) pointed out long ago, this initial understanding of the nature of beliefs and mind is not yet equivalent to a mature adult understanding of the mind. An understanding of the possibility of false beliefs will not allow children to make sense of situations in which people with access to exactly the same information still arrive at different, but equally legitimate, interpretations of the same information. A child with only an understanding of false beliefs would assume that only one interpretation could possibly be right, and others must therefore be wrong. To accommodate the experience of interpretive diversity given the same information, while maintaining the assumption of a stable, independently existing external world, children slowly modify their understanding of the process of knowledge acquisition to achieve an understanding of the interpretive nature of knowledge. So when children encounter situations in which different beliefs are apparently based on the very same information, they must revise their earlier understanding that beliefs depend on the information one is exposed to (i.e., false belief understanding) and develop an interpretive understanding of the mind (Carpendale \& Chandler 1996; Chandler \& Lalonde 1996; Lalonde \& Chandler 2002). This early insight into the interpretive nature of knowledge is a step toward a more mature understanding of mind, but there is still further development in understanding how knowledge is acquired during adolescence and adulthood (Carpendale \& Chandler 1996; Chandler et al. 2001). Proponents of the dominant theories have been notably quiet about what happens in development after the child's fifth birthday. However, research that explores whether 5-year-olds can use simple false belief knowledge to make inferences about their own and others' perspectives finds that they singularly fail to do so (Varouxaki et al. 1999).

In addition to the content of talk about the mental world, the approach we are proposing also implies that the nature of the parent-child relationship should be important in the development of children's social understanding through facilitating children's understanding of the events and human activities being talked about. We turn to this implication of our approach in the next subsection.

\subsection{The role of relationships in the construction of social understanding}

In this section we return to the issues illustrated by the sibling effect discussed earlier in this article (see sect. 1 and 
sect. 2.2). Any account of how the child comes to understand the psychological world must be able to explain a range of other sources of evidence showing that mental state understanding is significantly correlated with factors in the child's social environment, such as attachments, parenting styles, and parent-child communication. In this section we argue that the importance of communication in either facilitating or hindering the understanding of other perspectives directs our attention to the nature of communication within different relationships. The sibling effect is important because it is a marker of the types of process hypothesized here, but it is only one such marker. Dunn (1996a) has repeatedly reminded us of the important role of relationships in social cognitive development. What is needed is a theoretical explanation of how relationships operate as a vehicle for the child's construction of the mind. The idea of the epistemic triangle is useful in explaining this development because it is through becoming aware of other people's beliefs, and coordinating these often differing perspectives with their own beliefs, that children develop an understanding of mind. Through such interaction and by confronting others' often differing beliefs about the world, children gradually construct a more complete understanding that increasingly coordinates their own experience with that of other people. It is partially the resistance or refractoriness of social interaction that stimulates the development of knowledge. Thus, we would expect that differences in the amount and nature of the social interaction experienced would be related to individual differences in infants' early social understanding, and to young children's further developing mentalistic understanding. We will discuss two ways of describing parent-child relationships: attachment and cooperation.

4.2.1. Affective engagement and mental state understanding. A body of research in which social relationships are shown to correlate with social understanding has been influenced by attachment and psychodynamic theories (Fonagy \& Target 1997; Hobson 1993; 2002; Meins 1997; 1999). Fonagy et al. (1997) found that securely attached children, as measured with the Separation Anxiety Test, a projective measure of attachment security, were more competent on theory of mind tasks than insecurely attached children. This positive relation between attachment security and false belief understanding has also been found with a Q-sort measure of attachment (Symons \& Clark 2000). In a longitudinal study, Meins (1997) found that children who were classified as securely attached at age 11 to 13 months were more likely than insecurely attached children to pass a false belief task at age 4 years, and more complex mentalizing tasks at age 5 years (Meins et al. 1998). A relation between attachment and early social understanding in infancy was also reported by Bretherton et al. (1979). These researchers found that children who were securely attached at age 12 months had used more protodeclarative pointing at age 11 months than other infants.

There have been a number of potentially compatible explanations proposed for this positive relation between secure attachment and social understanding. Meins (1999; Meins et al. 2001) suggested that security of attachment and social cognitive development are positively associated because parents of children who develop secure attachments respond sensitively and appropriately. To do this they need to think of their children as persons with thoughts and feelings and treat them in this way. That is, parents whose children develop secure attachments are "mindminded"; they tend to "treat their infants as individuals with minds, rather than merely entities with needs that must be met" (Meins 1999, p. 332). This way of interacting develops secure attachment, and it also exposes young children to talk about the psychological world. Meins's explanation for the connection between attachment security and social cognitive development is that the same parental characteristic that results in secure attachment also results in the "exposure of the infant to mental state language" (p. 337). She found that maternal child-centered (mindminded) language (but not mindminded language about other people) to 6-month-olds predicted children's false belief performance at age 4 (Meins et al. 2002). This evidence requires an explanation of how exposure to mental state language influences social development, which is what we have provided here.

At a level at which theories of affect and cognition are integrated, it is not too difficult to show how analyses of infant-caregiver affect are wholly compatible with data on parent-infant shared attention. Indeed, Hobson (2002, p. 147) argued that security of attachment, which is based on the mother's way of relating to the infant, may influence the infant's ability to engage with others, and it is this engagement that is essential in interaction in the "relatedness triangle" and in the development of thinking, especially thinking about the social world. From the vantage point of observations of interactions, Baldwin and Moses (1996) pointed out that internal working models consist of knowledge of other people and relationships. That is, infants develop expectations about other people based on primary relationships. Such views echo one another and fit into the framework of our general developmental theory, which extends beyond infancy. Perhaps we should not be surprised that such a way of viewing internal working models converges with Piagetian ideas about development being rooted in interaction because one likely source of Bowlby's ideas was Piaget's notion of schemes. Piaget (1945/1962, pp. 188-89, 206-207) wrote about the "affective schemes" or "personal schemes" that infants develop as "modes of feeling and reacting" to people in ways that sound like internal working models - the set of expectations infants build up about other people and how they will act. This is early sensorimotor, lived, practical knowledge about people based on expectations acquired through experience of how parents respond.

Research on the development of affective exchange in infancy has greatly illuminated key issues in infant development. The correlations between such exchanges and tests of later mental state understanding suggest a need for a broader explanatory framework. One possibility is that secure attachment is an indicator of a relatively cooperative parent-child relationship, and, as we will argue in the next section, this is the type of relationship that, according to constructivist theory, facilitates the development of knowledge.

4.2.2. Cooperation versus constraint. Exploration of the nature of parent-child relationships has led to interest in the influence of parenting style on social cognitive development (Astington 1996; Vinden 2001). Ruffman et al.'s (1999) data show that parents who reported that they dealt with disciplinary situations by asking their child to "reflect 
on the victim's feelings" (p. 406) had children who were more advanced in false belief understanding. In addition, cooperative interaction is related to and may facilitate the development of children's social understanding. Dunn and colleagues (1991) found that cooperative sibling interaction was related to successful false belief explanations. Also, Brown et al. (1996) reported that cooperative interactions with friends and siblings were related to children's frequent use of mental state terms. Such evidence needs a theoretical explanation.

If, as Chapman (1991; 1999) argued, knowledge of the world develops through coordinating other people's perspectives with one's own within the epistemic triangle, then aspects of relationships and communication that facilitate our understanding of other people's perspectives should have a positive effect on development. An aspect of relationships that was important in Piaget's (1932/1965b; 1977/ 1995) work on moral judgment is the degree of constraint versus cooperation present in relationships. This analysis is relevant for our discussion because development, according to Piaget (1932/1965b), is facilitated by relationships of cooperation and mutual respect and hindered by relationships of constraint and unilateral respect; cooperation facilitates understanding. Cooperative relationships have the potential of approaching Habermas's (1983/1990) conception of the ideal conditions of unrestrained communication, allowing all participants to understand each other's positions fully and arrive at solutions to conflicts that everyone can agree with. In our terms, if understanding is gradually constructed by the child within triadic interaction, then it follows that the extent and nature of cooperation between the child and others are important.

It follows from a constructivist perspective that cooperative relationships, allowing free communication between parents and children, should facilitate the development of an understanding of mind. Parents differ in how much they feel obliged to justify their positions and listen to their children's perspective and, thus, in how much cooperation they allow or encourage in their relationships with their children. Relationships among parents and children that are more cooperative than constraining should facilitate children's understanding of other people's points of view. This interpretation is consistent with research showing that parental styles (Baumrind 1991) differing in the extent to which parents reason with their children differentially affect child development. Cooperative relationships are the ideal context for the development of knowledge, but this alone is not sufficient. Talk about a substantive connection to the world is also required (Döbert 2004) - in this case talk about people's activity in terms of their goals, beliefs, and desires. Various experiences such as cooperative peer interaction in the context of role enactment and shared pretense may contribute to the development of mental state understanding largely due to the stimulation of interaction and conversation. ${ }^{9}$

Of course, these correlations do not allow us to draw causal conclusions. The parent-child relationship is a system that is influenced by characteristics of both the child and the parents. It is important to acknowledge that relationships involve the child as well as the parent, and, although the influence of parents tends to be emphasized, that characteristics of the child also influence the nature of the parent-child relationship (Bell 1968; Symons \& Clark 2000). Parents' ability to engage with their child may be in- fluenced by their beliefs about parenting (e.g., Baumrind 1991; Ruffman et al. 1999; Vinden 2001) and emotions (Hooven et al. 1995), as well as by the parents' level of stress, depression and psychiatric disorders, patience, social support, socioeconomic circumstances, and education.

On the other hand, the extent to which parents can be cooperative and can support the child's reasoning will partly depend on, and interact with, the child's abilities and characteristics. The child's developmental level may influence the nature of the relationship because children who are more advanced in language and social cognitive development may be easier to interact with in a cooperative manner. ${ }^{10}$ Individual differences on the child's part may vary from extremes such as autism, ${ }^{11}$ which severely restricts the child's ability to engage in triadic interaction as evidenced by the lack of protodeclaritives at 18 months of age (Hobson 1993), to differences in ability to focus and maintain attention (e.g., hyperactivity, impulsiveness, distractability). Blindness also makes joint attention more difficult to achieve and for that reason seems to hinder the development of social understanding (Minter et al. 1998; Peterson et al. 2000). We agree with Hobson (1993; 2002) that such factors on the child's part influence the ability to engage in interpersonal relatedness, as well as the nature of that interaction, and it is such interaction that is essential in social cognitive development. In this target article we have restricted our analysis to the study of human development, but the same principles could be extended to nonhuman primates. $^{12}$

\section{Conclusion and future directions}

What benefits can be gained by adopting a constructivist approach to the development of children's social understanding? For a start we have summarized examples of a voluminous literature that relates an understanding of mental states to a range of social experiences. Traditional accounts of "theory of mind" can only go so far in adding to the list of social influences on the child simulator/theoretician before coming to realize that a social dimension has to be incorporated into such theories. The theory presented here sets out to do two things. First, it attempts to establish from the start that social processes are a necessary part of any account of how children come to construct an understanding of their social world: from infant dyadic interaction through to complex social skills beyond simple false belief understanding. Second, it has revisited and reworked the constructivist account of development and used this to reassert its central focus on action and the potential for examining knowledge acquisition as a social process. For us, a constructivist approach to understanding the child's grasp of the social world is the only possible solution to the impasse between individual and social perspectives on social understanding and the problem this reveals in the assumption that the "theory of mind" metaphor makes about the development of knowledge and meaning: that the child has to learn about the workings of the mind through inference or introspection. For us, following Wittgenstein, "as a human activity "meaning' is best thought of as a verb, not a noun" (Shotter 1978, p. 46).

By providing a framework that integrates social and cognitive processes, the analysis presented here calls for new directions in research based on the relations between particular forms of interaction and the development of social 
knowledge. Here we suggest some required shifts in the research agenda, as a means of illustrating how the implications of our position are very different from those offered by theorists within the "theory of mind" tradition. We refer to five areas of urgently needed research.

This research agenda begins in infancy with the study of the development of "shared meaning" (Chapman 1999, p. 34). This is one of the most difficult and important problems in developmental psychology. We have pointed out flaws in other approaches and provided a firm foundation and the tools with which to build an account of the development of infants' ability to grasp others' referential intent (see also Müller \& Carpendale 2004). But we acknowledge that the sketch we have provided is, as yet, partial. Researchers must begin by addressing the process by which the infant engages in interaction and joint attention. In doing so, we suggest that longitudinal naturalistic observation of infant-parent interaction and infants' joint attention would complement the current experimental research (e.g., Moore \& D’Entremont 2001). Ironically, such naturalistic research was more common 25 years ago (e.g., Bates et al. 1976; Lock 1978; Schaffer 1977). What is needed is a detailed analysis of infants' interaction and the emergence of social referencing, gaze following, and pointing, as well as a study of the context of the ongoing sequence of interaction in which the act is embedded. Some contemporary research of this sort does already exist (Reddy 1991) and we suggest that such work may help solve the riddle of how self-other differentiation and coordination emerge during infancy. According to the view adopted here, and in contrast to the position held in the "theory of mind" accounts of infancy, "the child's understanding is not just a matter of recognizing a correspondence between the mother's words and reality, but of grasping her referential intent in that situation - knowing what one is meant to attend to in response to her words and gestures" (Chapman 1999, p 34; emphasis in the original).

Second, we need to move from the intriguing findings relating individual differences in the social experiences of infants and their social understanding to research that explores the processes involved. For example, the findings of Meins et al. (2002) are intriguing, but how is it that mothers' tendency to talk about their infants in psychological terms is positively associated with their child's understanding of false beliefs almost four years later? What is it about the nature of these parents' interactions with their infants that correlates with the development of social understanding? Such research turns our attention to the nature of parent-child interaction and developing talk about the psychological world, and our theory provides a framework for this research. For example, such studies should examine how the criteria for the use of mental state terms are displayed (Turnbull \& Carpendale 1999a; 2001). Differences between families in the ways in which criteria are made evident could also be studied. It is important to remember that researchers studying talk about the psychological world should be concerned not just with mental state terms but more broadly with talk about human activity (see sect. 4.1).

Third, and related to the points above, the gradual view of development implied in an activity-based theory such as ours means that researchers need to study forms of understanding even before children are able to explicitly explain false beliefs. One way to approach this issue is by manipulating the tasks to discover what factors facilitate young chil- dren's reasoning within the context of the assessment procedure. This type of research - and a considerable amount of such research has been conducted (e.g., Lewis \& Mitchell 1994) - should be used to think about the nature of development, not merely to search for "early competence." This would involve an extension of research using novel approaches to assess children's grasp of an actor's beliefs. For example, Call and Tomasello's (1999) nonverbal false belief procedure could well be used to tap early forms of understanding, just as it has been used to reveal a competence in deaf children (Figueras-Costa \& Harris 2001; see also Carpenter et al. 2002). There is still scope to make a closer inspection of how children respond during traditional tests of false belief. For example, an interesting source of evidence of an early form of understanding comes from within the theory-theory and is referred to as "implicit knowledge" of false belief (Clements \& Perner 1994; Clements et al. 2000; Garnham \& Ruffman 2001). This work seems to demonstrate that young children, even slightly before their third birthday, respond to a prompt in a false belief test by looking in the direction of the correct container, even though these same children then go on to fail a more standard verbal question. Clements and Perner (1994) describe this knowledge as implicit because it cannot be verbalized. It has more recently been referred to as "nonverbal theory of mind" (Ruffman 2000), but we point out that although the child's response is not verbalized it is in response to a verbal prompt. Not only might research on this effect illuminate potentially interesting processes by which children come to construct an understanding of the false belief task (e.g., the nature of the criteria they use), it would also free theory-theory from the contortions it has to make to incorporate such evidence into its developmental account.

Fourth, we need to explore more closely just what it means for a child to "have" an understanding of belief, in order to move beyond overly simple accounts of theoretical transitions at age 4 years. As well as expecting gradual development in different forms of understanding, we also predict and would investigate unevenness in development between different areas of children's experience. Some children may have more experience and therefore more understanding of particular aspects of social understanding. For example, in a recent study, Peskin and Ardino (2003) found that children's performance on a false belief task in which an object is moved to a new location without the knowledge of the protagonist ("unexpected transfer task") is positively associated with success in a hide-and-seek game, whereas children's performance on a false belief task in which a container has unexpected contents ("unexpected contents task") is positively associated with success in keeping a secret from one of the experimenters about a birthday cake wrapped in a bag. Such data are clearly compatible with a constructivist account.

Fifth and finally, and as stated above, researchers need to move away from age/stage fixation to explore what happens to children after age 4 years. For a start, children cannot necessarily use the knowledge they demonstrate in the standard false belief test. A recent study by Maridaki-Kassotaki et al. (2003) found that in Greek, in which two terms are used synonymously to mean "to look for," a version of the false belief test with one verb appeared to facilitate 3year-olds' performance, whereas its synonym hampered performance in 5-year-olds. Such data reveal that children's 
understanding of questions about beliefs is mediated by their understanding of verbs referring to mental states. Further work along these lines would reveal more about the process by which children come to acquire the subtleties of a mature understanding of mind - particularly knowledge and skills that make up the complexity of a culture's psychology (Lillard 1998). The approach put forward here stresses the need to explore the matrix of social relationships involving transactions between the child and others and in which the child constructs social understanding.

\section{ACKNOWLEDGMENTS}

In developing the ideas presented in this target article we have benefited from discussions with Ulrich Müller, Bill Turnbull, and Tim Racine. We also thank Chris Moore, Vasu Reddy, and the referees for helpful comments on an earlier draft.

\section{NOTES}

1. It is not clear that an enculturation approach is directly championed by any theorist within the "theories of mind" literature, but such an approach is discussed by many (e.g., Astington 1996; Astington \& Olson 1995) and sometimes attributed to Wittgenstein or Bruner (1990).

2. Rogoff (1997, p. 266) criticized internalization approaches and argued instead for "development as a process of transformation of participation." Although there is much that we agree with in Rogoff's approach, her view of internalization involves either the transmission or acquisition of information (corresponding to collectivism or individualism, respectively) - both being examples of what Overton (1994; 1998b) termed a "splitting" or "isolation" strategy. Rogoff (see also Matusov 1998) critiqued a particular view of the nature of internalization. We agree with the critique. However, there is a second view of internalization (Lawrence \& Valsiner 1993), according to which internalization involves the child's reconstruction of knowledge rather than transmission. Interaction is internalized (or "interiorized," in Piaget's terminology) to the extent that the child can implicitly perform the act and does not actually have to enact the activity (Carpendale et al. 1996; Chapman 1991; 1999).

3. There are other positions such as Thelen and Smith's (1994; Thelen et al. 2001) dynamic systems approach that are consistent in many ways with the approach we take. Thelen and Smith's account is similar to Piaget's theory in emphasizing the practical and embodied nature of cognition. And Chapman (1992; see also Boom 2004) suggested that Piaget's theory was an early theory of self-organization. However, there may also be important differences. In Thelen and colleagues' (2001) explanation of the A not $\mathrm{B}$ error they appear to assume objectivity, whereas that is what Piaget tried to explain (Müller \& Carpendale 2001). This suggests that they started from a different beginning point in development.

4. We cannot survey here the extensive literature on Wittgenstein's private language argument, but we do need to at least respond to Kripke's (1982) interpretation. Kripke began from Wittgenstein's claim that all rules are up for interpretation. This is the paradox that "no course of action could be determined by a rule, because every course of action can be made out to accord with the rule" (Wittgenstein 1953/1968, para. 201). From this, Kripke derived the radically skeptical position that there can be no such thing as rule following, and this also applies to language since word use is rule following. This implies that the apparent meaningfulness of language must be an illusion and language must be meaningless. Kripke ended up in this radically skeptical position because he refused to give up the idea that rules are interpreted. But Wittgenstein was, in fact, arguing against this external explanation that there is a middle step of interpretation in applying a rule. Ironically, Wittgenstein was setting up the paradox that the range of interpretation is infinite to show that rule following cannot consist of interpretation - that "interpretations by themselves do not determine meaning” (para. 198). In the same section that Kripke focused on, Wittgenstein went on to write, "It can be seen that there is a misunderstanding here. . . What this shows is that there is a way of grasping a rule that is not an interpretation" ( para. 201). Then, in paragraph 202, "And hence also "obeying a rule’ is a practice” (McDowell 1984; Russell 1987). Wittgenstein's position is internalist: that is, it is not possible to separate the rule and the application (ter Hark 1990, Chap. 3).

5. Triadic interaction here refers to interaction involving self, other, and the world, not to interaction among three people.

6. However, Trevarthen took an innatist approach to explaining this development, whereas we do not.

7. We recognize that reference is a controversial issue (Putnam 1988). To be clear about our position, we do not consider reference to involve a dyadic relation between a sign and the thing referred to. This would seem to require a mechanistic view of meaning in which meaning is assumed to be attached to representations (Goldberg 1991). Instead, we consider reference to involve a triadic relation between the self, others, and an aspect of the world (Carpendale 1999b; Chapman 1991; 1999; Sinha 1999). Thus, meaning is not fixed to signs, but signs are used to direct others' attention and shared meaning is achieved through ongoing social interaction (Turnbull 2003; Turnbull \& Carpendale 1999b).

8. Variability in children's performance on false belief tasks is reminiscent of similar debates in other areas of research. The most well-known example is criticism of Piaget's theory concerning "horizontal decalage." The standard interpretation of Piaget's theory was as a theory of mental logic, according to which reasoning involves the application of a logical rule. A prediction derived from this interpretation is that, once a child has developed such a rule, he or she should be able to solve all problems based on the same underlying logical rule. It is well known, of course, that there is considerable evidence of variability in children's performance on tasks that are all apparently based on the same logical principle. The research literature provides other examples of similar difficulties in explaining evidence of variability in performance on different tasks that apparently should all be assessing the same competence. For example, in research on Kohlberg's theory of moral development, much more variability in the stage of moral reasoning employed was found than had been predicted by Kohlberg (e.g., Carpendale 2000). A particularly good example of the same sort of issue arose in the role-taking literature (e.g., Chandler 2001).

As argued elsewhere (Carpendale et al. 1996; Chapman 1987b), horizontal decalage is only problematic when it is assumed that Piaget's theory is a theory of mental logic. From the perspective of an interpretation of Piagetian theory emphasizing the origin of knowledge in action, horizontal decalage is not a problem (Chapman 1987b; 1988; Lourenço \& Machado 1996). Instead, it should be expected. Similarly, variability in performance on different false belief tasks is only a problem because it clashes with a common and implicit assumption about the nature of reasoning - that is, the view of reasoning as being based on the development of rules or principles which are then applied to problems to generate solutions (in domains such as moral reasoning or reasoning about the physical world, and now the social world).

9. Pretend play has been considered as a possible facilitating context for the development of social understanding, that is, as a "zone of proximal development" (Lillard 1993a; Youngblade \& Dunn 1995). The overall amount of pretend play has not been found to be associated with false belief understanding, but false belief understanding is associated with specific types of pretend play: when children make joint proposals in their pretend play and when they explicitly make role assignments to themselves and their partners in play (Astington \& Jenkins 1995). Also, young children's tendency to role enact is associated with belief understanding seven months later (Youngblade \& Dunn 1995). From our perspective, we would expect that increased social understanding would facilitate children's ability to engage in cooperative pretend play, and that this social interaction could also serve as one context, among others, for further social development. We 
would not expect that any facilitative effect would depend only on the fact that pretense is involved, but rather also on the fact that such situations would require cooperative interaction in which children must coordinate their activity toward shared goals. This would require talking about human activity and the need to reach mutual understanding because the children are enacting some event together.

10. In considering the role of the child's abilities we recognize that our approach needs to be integrated with domain general approaches to children's reasoning about the mind (e.g., Frye et al. 1995a; Gordon \& Olson 1998; Mitchell \& Riggs 2000). The child must have an ability to pay attention to important aspects of social interaction and must be capable of achieving some distance between himself and the situation so he can reflect on it rather than act impulsively (Moses 2001). Furthermore, reasoning about situations that are not immediately present would require the ability hold in mind and to imagine aspects of situations (Harris 2000). Clearly, there is some distance to go in developing this aspect of our approach, but we suggest that a likely candidate for further study would be attentional capacity, which in Chapman's (1987b) approach has a role in understanding and reasoning.

11. Concerning autism, our position is consistent with Hobson's $(1993 ; 2002)$ view of autism as due to a disruption in the child's ability to engage in affective interaction. In this article we have spelled out in further detail the nature of this interaction beyond infancy and the role of language in social cognitive development. However, we have not applied the issue of autism to our analytic framework because it is associated with so many other social and cognitive problems and differences from the typically developing population that no conclusive statement can be made here.

12. This focus on the role of relationships in development, as well as what the child brings to the relationship, allows us to think about both atypical development and research with nonhuman primates. Here our approach is consistent with Tomasello's (1999b) position that what is required in typical social cognitive development is both normal neurological development as well as the right social and cultural conditions. In children with autism we see biological abnormalities hindering the children's ability to engage with others in ways that are essential to normal social cognitive development, even though they have access to normal human social interaction (Hobson 1993). "Enculturated" apes - that is, chimpanzees and bonobos raised in a human linguistic environment - do develop more social cognitive and language skills than wild chimpanzees, but they can only go so far - not much beyond the level of a 2-year-old human child (Savage-Rumbaugh et al. 1993).

\section{Open Peer Commentary}

Commentary submitted by the qualified professional readership of this journal will be considered for publication in a later issue as Continuing Commentary on this article. Integrative overviews and syntheses are especially encouraged.

\section{What's new about social construction? Distinct roles needed for language and communication}

Janet Wilde Astington

Department of Human Development and Applied Psychology, Ontario

Institute for Studies in Education, University of Toronto, Toronto, Ontario M5S 1 V6, Canada. jwastington@oise.utoronto.ca

http://fcis.oise.utoronto.ca/ jwastington/

Abstract: Carpendale \& Lewis's (C\&L's) theory falls in with an existing set of theories that children's understanding of mind is collaboratively constructed in linguistically mediated social interaction. This social constructivist view needs to be clear about the complementary contributions of the child and of the social environment. I distinguish between the child's individual linguistic ability and the dyad's social communication, proposing that each makes a contribution to theory-of-mind development, differently balanced in different individuals.

Rejecting two contrasting paradigms, Carpendale \& Lewis (C\&L) offer their explanation of children's theory-of-mind development as a supposedly new alternative to existing theories. On one hand, they reject explanations that posit theory development, simulation, or innate modules because these are too focused on autonomous individual development. On the other hand, they reject enculturation explanations because, in their view, such explanations allow the child no active role. The internalist theories that they reject are well described and supported in the literature, whereas, as they concede, the externalist view is hard to find (perhaps it is best, if briefly, exemplified in Rogoff et al. 1993).

Social construction. I agree with C\&L that we need an explanation integrating the individual and social aspects of development; and I am sympathetic to their account, accepting it as an alternative to the internalist, individualistic explanations and the externalist, passive enculturation explanation, all of which the authors explicitly reject. I find it harder, however, to regard theirs as a particularly novel approach. Although some terms and details may differ, it is best located within an existing group of social constructivist explanations of children's developing understanding of mind (e.g., Boyes et al. 1997; Dunn 1996b; Fernyhough 1996; Garfield et al. 2001; Harris 1999; Montgomery 2002; Nelson 1996; Shatz 1994). I would also place my own views here - let me resolve, in passing, the ambiguity that $\mathrm{C} \& \mathrm{~L}$ detect in my earlier writing (e.g., Astington 1996; Astington \& Olson 1995). I do not believe that children's theory of mind develops by a process of passive enculturation. In my view, it is an error to assume that "cultural concepts can be acquired from participation in a social context simply because they are manifest there" (Astington \& Olson 1995, p. 186).

Although I accept that there is need for "an alternative theoretical account that is more consistent with the accumulating evidence of the important role of social interaction in the development of children's social understanding" (target article, sect. 2.2, penultimate para.), I would argue that a number of such alternative accounts already exist in the literature. C\&L share with Nelson (1996) and Montgomery (2002) the Wittgensteinian idea of acquiring meaning from use; they share with Harris (1999) and Fernyhough (1996) the idea that children are exposed to variation in belief as they enter into conversation with others; they share with Dunn (1996b) a focus on the relations between people; and so on. 
Hence, C\&L's proposal falls in with an existing set of theories that children's understanding of mind is collaboratively constructed in linguistically mediated social interaction. Their account accords a central role to triadic interaction involving a dyad - the child and another person - communicating about some object or aspect of the world. What is needed is a more detailed and precise account of the mechanism whereby this leads to an understanding of mind. From a social constructivist perspective, it is important to be precise about those abilities of the child that allow for participation in the constructive activity, lest we slip back into passive enculturation. Perhaps by distinguishing between children's individual linguistic ability and their participation in dyadic linguistic interactions (although obviously these two are related), we will be able to formulate a social constructivist view that recognizes the contribution both of the child and of the social environment.

Language and communication. It is problematic that C\&L use three terms somewhat interchangeably: social interaction, communicative interaction, and language. In my view, it is important to distinguish clearly among these. One could use the term "language" to refer to the child's individual linguistic ability and "social interaction" to refer to the joint activity within triadic interaction, reserving the term "communicative interaction" for discourse-based social interaction (although this last may be less than satisfactory because it obscures the importance of nonverbal communication). My point is that, defined in this way, language and social interaction/communication may play different and complementary roles in theory-of-mind development and, further, that there may be both individual and developmental differences in their relative importance. Some evidence exists in support of this suggestion.

First, deaf children with deaf parents develop false-belief understanding within the typical time frame, whereas deaf children with hearing parents do not do so (Peterson \& Siegal 2000). Both sets of children engage in triadic social interaction, which is communicative even if nonverbal. However, only the children with deaf parents develop language - sign language - within the typical time frame. This suggests that the child's individual linguistic ability plays a role in the development of theory of mind independent of that played by social communication.

Second, children with autism have deficits in theory of mind and in the development of communication and language skills. High-functioning children with autism do develop false-belief understanding, but later than typically developing children do. Moreover, those who do pass false-belief tasks have a higher level of general language ability than that of typically developing children who can pass false-belief tests (Happé 1995). This suggests that the autistic children are using their individual language skills to compensate for their lack of social communicative interaction (Tager-Flusberg 2002).

Third, there is evidence for the interaction of family size (indicative of the amount of child-child social activity) and individual language ability in theory-of-mind development (Jenkins \& Astington 1996). We showed that the false-belief test performance of children with lower language ability and two siblings was equal to that of children with higher language ability and no siblings, whereas children with lower language abilities and no siblings performed more poorly. This suggests that individual language skills and social interaction both contribute to theory-of mind development and that either one of these can compensate for a deficit in the other.

Fourth, in a training study designed to develop false-belief understanding (Lohmann \& Tomasello 2003), positive effects were found in two conditions: conversation about deceptive objects (using no mental terms and no syntactic complementation in the discourse) and specific training on the syntax of complementation (in the absence of deceptive objects). Moreover, the largest effect was found in a condition where these two were combined (conversation and complements). Again, this suggests that social interactive discourse and individual language abilities make somewhat independent contributions to theory-of-mind development.
Different routes to common outcomes. In conclusion, I would argue that theory of mind is a fundamentally important aspect of human development which is likely to be overdetermined. That is, it is likely that there is more than one cause of its development - individual linguistic ability and social interaction are two discussed here - with different balances in different individuals, and perhaps with the balance shifting over developmental time, as Hughes et al. (2002) suggest.

\section{The role of social experience in advanced social understanding}

\author{
Robin Banerjee \\ Department of Psychology, University of Sussex, Falmer, Brighton BN1 9QH, \\ United Kingdom. R.A.Banerjee@sussex.ac.uk \\ http://www.cogs.susx.ac.uk/users/robinb
}

Abstract: Carpendale \& Lewis (C\&L) rightly emphasise the central role of social interaction in the development of children's understanding of mind. Further support and justification for their theoretical focus are provided by research on advanced reasoning about socio-emotional and socio-motivational processes. Variability in social experience can explain both developmental change and within-age-group differences in such social understanding.

In the constructivist framework presented by Carpendale \& Lewis $(\mathrm{C} \& \mathrm{~L})$, a central role is assigned to children's social experience as an essential foundation for gradual social-cognitive development. The authors indicate the potential significance of findings regarding relatively advanced (beyond five years of age) reasoning about the psychological and social worlds, especially in view of evidence that simple false-belief measures have only limited value for predicting variability in children's everyday social behaviour. Brief reference is made to children's understanding of the interpretive nature of knowledge (e.g., Carpendale \& Chandler 1996), but the analysis would be strengthened by considering additional evidence regarding primary schoolchildren's cognition about the emotional and motivational processes involved in everyday social interaction. Both developmental change and within-age-group differences in this social understanding reflect children's experiences of social interaction. Indeed, Dunn's (1996a) insightful reminder to address the role of social relationships in social understanding should hold through the course of childhood socialcognitive development.

Research on social cognition has demonstrated many developmental changes beyond the age of five years. Taking emotion understanding as an example, we see primary school children learning about self-regulation (in terms of both private coping and public display), about emotional ambivalence, and about complex emotions involving reflections on the self, on others, and on social standards (e.g., Meerum Terwogt \& Stegge 1996). Similarly, researchers have developed a range of advanced theory-of-mind tasks covering, for example, children's reasoning about second-order false beliefs (e.g., Sullivan et al. 1994), about faux pas (BaronCohen et al. 1999), and about irony, sarcasm, and double bluff (Happé 1994). There is also evidence of change in children's broader understanding of people, such as in their conception of dispositions or traits (e.g., Yuill \& Pearson 1998). Research on these topics may provide strong evidence concerning the role of social interaction in children's social understanding.

One feature of advanced social understanding emphasised by Carpendale and Chandler (1996) concerns the fact that different people may hold varying beliefs about the same perceptual stimuli. In that paper, the authors found that only from around seven or eight years of age did children appreciate the validity of multiple interpretations of a range of stimuli (e.g., in the domains of lexical ambiguity, ambiguous line drawings, and ambiguous referential communication). It is reasonable to suppose that this 
development must at least in part reflect children's growing experience of situations that lend themselves to varying interpretations and evaluations. In particular, peer interactions are likely to encourage children to gain the mature insight that exposure to the same information can lead to varying beliefs about the world.

Recent theoretical and empirical work on children's use and understanding of self-presentation is consistent with this emphasis on peer interactions. Self-presentational behaviour involves an attempt to control the way one is evaluated by others, and success in this goal depends largely on an appreciation of how characteristics and events are represented by others. Hence, as children grow older and peer-group acceptance becomes an increasingly important goal (e.g., Parker \& Gottman 1989), others' beliefs must be judged with regard not just to their informational content but also to their evaluative content. Consistent with this evidence, Banerjee and Yuill (1999) found that children's spontaneous identification of self-presentational motives for deceptive emotional displays (e.g., not wanting to appear babyish or stupid) emerged at around eight years of age, lagging behind their identification of prosocial motives. Furthermore, such identification of self-presentational motives was found in a separate study to be associated with the attribution of embarrassment (Banerjee 2002a). In a similar vein, children's tendency to make appropriate use of information about an audience's preferences when choosing between selfdescriptive options also increased at eight years. Importantly, within-age-group variability in this tendency was positively associated with a sociometric measure of reciprocated peer nominations (Banerjee 2002b). Although work on this topic is in its early stages, there is good reason to suppose that children's reasoning about self-presentational motives and related social emotions, such as embarrassment, depends on their experience of specific types of social interaction. Indeed, this argument is consistent with Higgins and Parsons's (1985) broader discussion of how changes in children's social lives - including the increasing exposure to social agents with different dispositions, intentions, and preferences - are likely to play a critical role in shaping their cognition about the social world.

The appreciation of evaluative beliefs is just one of many aspects of social understanding that continue to develop in middle childhood and beyond. Researchers have already begun to identify social experience - involving various social agents (e.g., parents, siblings, and peers) - as playing a significant role in this developmental process. Two important related issues should be considered in any examination of this area. First, with respect to ambiguities about causal direction, we must recognise that children's social understanding necessarily develops in the context of their social experience. Even if we were able to specify contextgeneral prerequisites (e.g., understanding recursive mental representations), it simply does not make sense to talk about children's reasoning about self-presentation, or faux pas, or embarrassment without emphasising the social dimension. In these and other instances of reasoning about socio-emotional and socio-motivational processes, social understanding has to develop out of, and feed back into, children's social experience. Second, a major strength of the theoretical orientation presented by C\&L lies in its potential ability to explain both between- and within-age-group variability. Such variability has been observed in early mental-state reasoning, and researchers have found predictive value in children's interactions with parents, siblings, and peers. Now we are beginning to identify substantial variability in responses to more advanced tasks, not just between younger and older primary school children, but also within any given classroom of children. C\&L's framework provides further impetus to investigate the social experiences that give rise to these differences. Such work will help us meet the further challenge of predicting and intervening in the development of problems in social understanding.

\section{Even an "epistemic triangle" has three sides}

\section{John Barresi and Chris Moore}

Department of Psychology, Dalhousie University, Halifax, Nova Scotia B3H 4J1, Canada.jbarresi@dal.carmoorec@dal.ca

http://jbarresi.psychology.dal.ca

Abstract: By focusing primarily on communication between adult and child and on adult-set criteria for appropriate action, Carpendale \& Lewis's (C\&L's) account of the development of social understanding in the epistemic triangle tends toward an enculturation view, while diminishing the role of individuals. What their proposed mechanism fails to acknowledge is that the two agents in the epistemic triangle necessarily have independent perspectives of the object and of each other.

We strongly agree with Carpendale \& Lewis (C\&L) that the child develops social understanding primarily through engagement with other individuals in what Chapman (1991) called the "epistemic triangle." But we fail to see how their use of this triangle provides them with a way to chart a middle course between the Scylla of individualism and the Charybdis of enculturation. When it comes to describing actual mechanisms of development in the epistemic triangle, we find their account both incomplete and potentially biased in the direction of enculturation.

C\&L's discussion of mechanisms involved in the development of social understanding tends to use a particular interpretation of Wittgenstein's views on language learning. According to this account, it is socially implemented feedback involving behavioral "criteria" that shapes the child's use and understanding of language in general and mental language in particular. But if this is the primary mechanism for the development of social understanding of mental life, it fails to satisfy. Despite the authors' claims to the contrary, it does not differentiate their account from a general model of learning based on enculturation. For, even if particular triadic interactions are the locus of language and other learning, this mechanism appears to use teachers as carriers of linguistic and other social or cultural knowledge. Hence, the particular agents in the interaction, with their individual uniqueness, play an unclear role in the acquisition of language, and, presumably, in the development of social understanding in general.

The authors claim that their view is not an enculturation view because it allows for certain basic-level "shared practices" in social understanding of "seeing, looking, intentions, desires, and beliefs" that are fundamentally universal, hence not culture-relative. How this could save them from the socially constituted pole of enculturation is not obvious, unless it implies that the learning of such practices does not depend on language learning. But then, what does it depend on? One is tempted to answer: "a theory of mind"; but obviously this is not their answer. They also argue that they have a constructive account of development because the epistemic triangle is combined with the concept of criteria. Again their description here fails to explain adequately how the epistemic triangle plays a role different from that of criteria. This is apparent in their final summation: "We suggest that children understand talk about the psychological world in terms of the patterns of activity that are criterial for the use of such mental state terms - that is, the pattern of interaction for which we use these words" (sect. 3.2, last para.). Nothing is stated here about universal practices or of the role of epistemic states - just culturally defined criteria. It may be that a richer interpretation of Wittgenstein could save this account, one which recognizes first-person criteria for mental meanings to go along with public third-person criteria, but this is not the Wittgenstein that C\&L provide.

The nub of the problem is that C\&L fail to take seriously the triadic structure of the epistemic triangle. This structure involves three vertices (i.e., the infant, the adult, and the object) as well as three sides (i.e., the dyadic communicative relation between infant and adult, the infant's relation to the object, and the adult's relation to the object). By focusing on the communication between adult and infant and on adult-set criteria for appropriate action toward the object, they fail to recognize that the two agents 
in the epistemic triangle necessarily have independent perspectives of the object, and of each other.

The same problem arises elsewhere in their account, particularly in the section dealing with the infant's initial entry into the epistemic triangle toward the end of the first year of life. In this section they briefly describe our own theory of social understanding (Barresi \& Moore 1996). Because we talk of "first-person information" as one component entering into the epistemic triangle, C\&L claim that our model, despite pretensions otherwise, must be individualistic. But this claim both misrepresents our model and refuses to recognize an essentially individualistic aspect of the epistemic triangle - the two agents who, even in shared activity, necessarily have distinct intentions, goals, and perspectives in their relations with each other and to the object.

We believe that the account in Barresi and Moore (1996) is more sensitive to the structure of the epistemic triangle and to the mechanisms involved in social understanding than that given by C\&L. We argue that the infant first comes to understand mental phenomena by sharing with another individual an activity toward an object, whether that activity involves actions, emotional or motivational relations, or epistemic relations, all of which we treat as various forms of what we call "intentional relations." This sharing, whether initially achieved through behavioral or emotional contagion, conditioning, imitation, or any other means, provides the ground for developing a reflective representation of that form of intentional activity. The key point is that such a representation involves the joining together of both first-person information and third-person information about the common intentional relations involved in such triadic interactions.

We further suggest that in integrating information about two sides of the epistemic triangle (i.e., each agent's intentional relation to the object), in the context of an interaction about this common activity, the infant does not initially represent this activity as involving independent actions of self and other. Instead, at this early phase in development, the sources of information are not yet distinguished as originating from distinct individuals, and the understanding of the intentional relation is best described as one of understanding what "we" are doing. So, on our account, it is fundamentally an understanding of a shared activity, not one of understanding that self and other, as distinct agents, share in an activity. Nevertheless, it is from such initial understanding of common activities in triadic situations that the infant eventually develops an understanding of self and other as distinct agents with their own individual intentional relations. Moreover, it is always out of shared activity with others that advances in understanding of mental phenomena are first made (see Barresi \& Moore 1993). This is because it is only in such shared activities that the first- and third-person aspects of such mental phenomena may be recognized as belonging together, thus laying a foundation for eventually using either the first- or third-person aspect alone to recognize the mental state of self or other.

\section{Articulating the role of experience in mental state understanding: A challenge for theory-theory and other theories}

\section{Karen Bartsch and David Estes \\ Psychology Department, University of Wyoming, Laramie, WY 82071. bartsch@uwyo.edu estes@uwyo.edu}

\begin{abstract}
Carpendale \& Lewis's (C\&L's) proposal of a social interaction account makes clear the need for researchers of all theoretical orientations to get specific about how social experience influences children's developing understanding of mind, but it is premature to reject other theories, such as theory-theory, which also attribute a major role to experience.
\end{abstract}

Carpendale \& Lewis (C\&L) make clear that researchers, regardless of theoretical orientation, need to get specific about how chil- dren's social experiences affect mental state understanding. Drawing on an impressive array of studies showing that social experiences and social factors play a role in this important area of psychological development, C\&L argue that a new explanation of development is needed to account for these observations. Yet, few extant theories reject the descriptive claim that social experience and context matter. Theory-theory (Gopnik 1993; Gopnik \& Wellman 1992; 1994; Wellman 1990), for example, contends that children's experience in the relevant domain (presumably including people's psychological states and actions as revealed in social interactions) is the primary instigator of development, chiefly when children encounter phenomena that do not fit their current explanatory framework and must reconstruct that framework. It may be premature to reject current theories (and specifically theorytheory) without examining closely the implications of existing theories for the data.

We have previously noted that an understanding of some mental states seems directly dependent on observing what people say and do (Bartsch \& Estes 1993). False belief, the "neurotic" focus of theory of mind (TOM) researchers, can be recognized in real time only through such observation. I cannot recognize my own current belief as false, but I can witness someone else proclaim a belief I know to be false and then act on it. Perhaps one could eventually comprehend the representational (and thus imperfect) nature of beliefs merely by reflecting on one's own past false beliefs, but it seems likely that talking with others and watching their goofy actions based on erroneous convictions contributes to this recognition. Children's earliest talk about beliefs supports this supposition. For example, Adam (age four years, two months) explained that the dog barked on hearing footsteps because "She thought that was a tiger" (Bartsch \& Wellman 1995, p. 114). According to theory-theory, experience drives conceptual development; if experience concerning false belief occurs (in this important sense) only in social encounters, then it seems to us that theory-theory necessarily accords an important role to such encounters. Perhaps theory-theorists have not yet offered sufficiently extensive analyses of how experience (social or otherwise) translates into conceptual development, but that does not mean that such experience is any less than the primary engine of development on this account (see Bartsch 2002, for further discussion of the role of experience in theory-theory).

C\&L argue that the nature, as well as the fact, of the effects of social factors on mental state understanding mandates a new explanatory account. They point to murkiness across research results from studies employing different tasks and contexts: On some tasks, two-year-olds appear to engage in belief reasoning, whereas on others, only much older children do. We have encountered such findings in our own research. To examine children's use of belief understanding in persuasion, we administered hypothetical story tasks requiring them to select an argument that would persuade a story character (e.g., a child's parent) to do something, such as permit a child to get a puppy (Bartsch \& London 2000). Only children over seven consistently chose arguments relevant to the character's belief, such as "the puppy is quiet" in response to a parent's stated belief that puppies are noisy. However, in comparable tasks involving interactive dialogue with puppets, even four-yearolds consistently chose belief-relevant arguments (Bartsch et al. 2003). The interactive format not only increased children's attention to belief information, it seemed to squeeze belief reasoning even out of children who did not pass a "gold-standard" falsebelief task. This is precisely the sort of finding that C\&L regard as evidence that there is a gradual development of understanding that is dependent on social factors and social contexts.

Our interpretation is different. Despite the messiness of the empirical data, we think that it is true and useful to characterize the older children in our studies as having a concept of belief and the youngest children as not having the concept, consistent with a theory-theory perspective. We agree with C\&L that the variation observed across methodologies deserves close examination. The fact that young children sometimes succeed on tasks and older 
children sometimes fail is important and might hold clues about how development occurs. For instance, we suspect that the youngest children's performance on our interactive persuasion tasks is indeed socially scaffolded through the child's engagement in dialogue. The social context of persuasion, in which two agents are initially at odds, together with the proclamation of a belief in back-and-forth dialogue (e.g., "I think puppies are noisy!"), may be sufficient to allow the child to respond reflexively with the argument that "this puppy is quiet." But our suspicion is that the child responds to this situation without having much of a concept of belief, perhaps without any. However, such interchanges may provide important experience to children so that they eventually notice that responding to someone's stated belief with an argument relevant to that belief is more effective than when they respond with an irrelevant comment. This experience cannot be explained without an understanding of beliefs and their sources, so eventually the child adopts the more comprehensive theory. In this sense, we certainly agree with C\&L that close inspection of the social facilitators of development is essential for explaining the development of mental state understanding. But social facilitation effects are not necessarily at odds with theory-theory.

C\&L complain that theory-theory and other existing theories focus on cognitive architecture without reflecting on the social landscape. But surely any adequate constructivist account must address conceptual change in individuals and its implications for developments in social understanding and reasoning, even when all the scaffolding provided by the social landscape has been acknowledged. Theory-theory offers exactly what C\&L (target article, Abstract) say is needed, "a theory of development . . . that accords a fundamental role to social interaction, yet does not assume that children simply adopt socially available knowledge but rather that children construct an understanding of mind within social interaction."

\section{Why believe in beliefs?}

\section{Mark H. Bickhard \\ Department of Cognitive Science, Lehigh University, Bethlehem, PA 18015. mark@bickhard.namenhttp://www.bickhard.ws/}

Abstract: A central pillar of Carpendale \& Lewis's (C\&L's) argument is Wittgenstein's later work on language. I suggest that this support is not as strong as might be wished, and offer an alternative approach to their conclusion that language learning, especially of folk psychology, involves a socially embedded constructivism.

Two intertwined issues are the focus here: What is the status of belief talk? And how do children learn this "folk psychology" way of talking and functioning in the world? I would like to suggest some problems with a core set of arguments in Carpendale \& Lewis's (C\&L's) target article, and offer an alternative but convergent perspective on children's learning of folk psychology.

C\&L's discussion turns on Wittgenstein's later discussions and arguments concerning language and language learning, and is therefore only as strong as those discussions and arguments. But there are reasons for concern here. First, Wittgenstein was appropriately concerned with the normativities of language, with the "grammar" of language. ${ }^{1}$ However, his account of that normativity fails. His discussions in Philosophical Investigations (Wittgenstein 1958) and previous works regarding ever more complex language games and the forms of life that these might participate in, do not provide an account of normative emergence - complexity per se does not generate normativity - and leave readers with a "brute" normativity (Bickhard 2003; Shankar 1996; Summerfield 1996). This is not an adequate account of normativity, and that casts doubt on other aspects of Wittgenstein's account of language.

The private language argument, in particular, turns precisely on normativity as emerging only in the realm of the social. But this ignores, for example, the normativities of individuals' epistemological and representational relations with the world, including the social world, and including, in particular, the language games individuals are involved in. Wittgenstein slighted issues of epistemology until his last work, On Certainty (1969), and even then approached it solely from within a language framework. His failure to account for prelanguage normativities (and the normativities of prelinguistic animals, including human infants) constitutes a major deficiency.

An additional problem with Wittgenstein's approach, at least as generally interpreted, lies in his account of the learning of words for mental phenomena, such as belief. "Inner processes require outer criteria" is an oft-repeated slogan. There is clearly a sense in which this is correct: Something in the social interaction must be involved in the learning of any word. But what is the (normative) epistemological relationship between the individual (toddler) and those criteria? What is it that is being learned regarding, say, "belief"? A standard interpretation is that a complex nonmonotonic language game of criteria is being learned, one in which some criteria support the use of the term, while others, if present, may defeat that use, as when noticing that someone engaged in street theater defeats the primary criteria that they seem to be in pain. As this illustrates, however, that such learning must take place in terms of such criteria, slides into the assumption that what is learned must be constituted out of such criteria (however complex that constitution may be). This is not valid: If some internal constructive process is ongoing, and external (social) criteria serve as selections on that process, then what is learned - constructed need not be constituted out of those criteria at all. That we must learn from criteria, does not entail that criteria are what we must learn. ${ }^{2}$ The knowledge of how to "make it" as a frog is not constituted out of the survivals, deaths, and reproductions that were selected among the evolutionary constructions involved.

"Learning language as learning names," which C\&L (not to mention Wittgenstein) so rightly criticize, is in even worse condition, however: There is nothing unitary, no coherent kind of matter of fact, for belief talk to refer to. The argument here is the (extended) frame problem. I believe that large trucks can hurt me; I believe that trucks with stripes can hurt me; so can trucks with polka dots; and so on. There is an unbounded number of "beliefs" that I have, that anyone has, about trucks and their capacity to harm, as well as about almost everything else. Many of these beliefs are ones that I may have never thought of before, and yet they describe accurately what I believe. But there are too many of them to "fit" into a bounded skull, and too many of them to be learned by a toddler in a bounded time span (Bickhard 2001). Whatever else it does, belief talk cannot always or primarily refer to individuated beliefs. This I take to be convergent with C\&L's arguments. Note that the point holds regardless of the role of external criteria in the learning process.

What is belief talk, then? The central suggestion is that folk psychology, belief talk especially, is involved in making, breaking, discussing, evaluating, and so on, the epistemic claims and commitments that people make with each other, including those that others make with third parties, as well as those that each individual may be involved in directly (Bickhard 1998). Hence, if I claim, or if I behave in a way that presupposes, something to be true in the world, then I have a commitment to answer challenges to that presuppositional belief, perhaps by supporting the belief involved, or by supporting my legitimacy in that belief even if it turns out to be false, and so on. Most importantly, I do not have to have any explicit representational belief that the world is not made of Brie cheese in order to presuppose it, and, hence, to "believe" it. Therefore, I do not have to have any individuated inner process nor any individuated criterial learning in order to have such a belief. This belief is not likely to encounter challenges, but my general belief that "figures of authority are malevolent" might, and yet it too need not involve individuated processes or learning. It too can have the ontology solely of a presupposition of the way I live in the world. 
In this view, belief talk and, by extension, folk psychology talk in general cannot be learned as a naming game or as a mere internalization of social practices. It must be constructively learned as a form of coordination of modes of commitment and modes of explanation for the presuppositions of our interactions with each other and with the world.

\section{NOTES}

1. "Normativity" is used here in the philosophical sense of involving the possibility of being bad or wrong. It includes such oppositions as functional-dysfunctional, true-false, correct-incorrect, and so on. Regarding Wittgenstein and language, see, for example, Glock (1996) and Shankar (1996).

2. It is an empiricist assumption that it must be so constituted. In contrast, any action-based model of representation forces learning to be a constructivist process: The environment cannot impress successful action systems into an otherwise passive mind (Bickhard \& Campbell 1989).

\section{The contributions of the interdisciplinary study of language to an understanding of mind}

\section{Nancy Budwig \\ Department of Psychology, Clark University, Worcester, MA 01610. \\ nbudwig@clarku.edu http://www.clarku.edu}

\begin{abstract}
Carpendale \& Lewis (C\&L) emphasize the importance of viewing language as activity. In this commentary I push further their claim by highlighting how constructions, rather than words, are the appropriate unit of analysis. In addition, I suggest how a discussion of indexicality paves the way for a better understanding of how language provides a powerful tool for children's construction of mind.
\end{abstract}

Carpendale \& Lewis (C\&L) offer a provocative argument concerning children's construction of knowledge in and through social interaction. Although I am in large agreement with the general claims C\&L make, I aim to clarify their argument concerning the dynamic interplay between language and the development of an understanding of mind by touching on two inter-related issues: first, how functional linguistics can contribute to a better way to conceive of language, and second, how work from linguistic anthropology can contribute to an account of how language plays a guiding role in children's construction of an understanding of mind.

Beyond words: Constructions as the unit of linguistic analysis. One of the strengths of the target article concerns its emancipated conception of language as activity. Rather than buying into the tendency to view language solely in terms of its representational function, C\&L stress the centrality - borrowed from Wittgenstein - of viewing language as action. Nevertheless, the authors sometimes slip back into adopting jargon typically associated with the strictly representational view of language and could be encouraged to move beyond the focus on "words" by beginning to think of meaning in terms of the more dynamic notion of constructions (see Budwig 1998; Goldberg 1995; Tomasello 1998). Constructions are the typical unit of analysis for functional linguists, rather than the distinction between words and syntactic rules, because of their focus on patterns of usage. Simply put, constructions are best viewed as usage-based patterns and can be units of meaning that involve, for instance, bound morphemes (e.g., past tense-ed), words (e.g., doggie), or more abstract constructions such as "Animate Actor Xed the Y."

The relevance of looking at constructions for understanding children's construction of mind can be illustrated by some crosslinguistic work I have conducted examining children's early use of transitive and intransitive utterances. For example, American twoyear-olds have been noted to reserve, early on, transitive constructions to scenes describing their own (and sometimes others') intentional actions that bring about change (e.g., My build a tower). These same children switch to intransitive constructions to describe a scene involving goal-blocking, as, for example, when they intend to place a block on a tower they are building, but because the block is too large, the structure tumbles (It doesn't build). Careful analyses of such constructions offer insight into children's construction of mind, although mental state vocabulary has not been the focus of analysis (see Budwig 2002; Budwig et al. 2001).

The idea emphasized by functional linguists who use constructions as the unit of analysis is that linguistic forms themselves do not carry meaning in terms of re-presenting reality but rather, when fixed in particular scenes or symbolic fields, such forms gain a more dynamic meaning and thereby play a central role in structuring experience. To this extent, constructions as embodied units of meaning that link sign and symbolic field play a central role in mediating the interpretation of reality.

Indexicality and children's construction of mind. Although there is growing agreement that social interaction is an arena within which the unfolding of an understanding of mind occurs, little is known about the particulars of this process. C\&L begin to shed light on this issue by suggesting that relationships and talk itself provide fundamental resources for children. Further insight into how we might better understand the role of symbolic behavior in constructing culturally appropriate ways of understanding mental life could stem from current work in linguistic anthropology. Linguistic anthropologists start from the assumption that talk is a highly organized activity in which conversation unfolds in terms of highly structured units that universally segment the ongoing stream of action into orderly sequences related to culturally sanctioned ways of organizing social life (see Budwig 2003; Duranti 1997). Central here is the discussion of indexicality. As Ochs (1996) argues, linguistic constructions come to be habitually used in such ways that they come to index larger meaning units. The indexical use of language constructions provides communicative partners with signposts for how each partner grounds the interaction and thereby allows for the dynamic unfolding of meaning. Use of the particular construction comes to stand for a larger meaning unit.

Consider, for example, the use of desire talk in a crosslinguistic study of German and American caregiver-child dyads when the children were two years of age (see Moissinac \& Budwig 2000). The American caregivers were noted to use desire verbs in constructions either to inquire about their children's ongoing states (Do you want a tea cup?) or to access permission from their children to act in particular ways (Do you want me to pour tea?). In contrast, the German caregivers rarely grounded desire talk in terms of individuals' desires and, rather, used desire talk to construct joint activities and otherwise relate the children's actions to normative frames. For instance, one mother appealed to joint desire as she realized it was time to clean up the blocks, saying to her child: "Wollen wir die mal einsammeln (Do we want to collect them)?" The claim here is that the regular use of desire constructions, and more specifically, the way in which particular desire constructions linked up in patterned ways with larger meaning units, provides a powerful point of entry for children to tacitly begin to work on ways of seeing that are culturally sanctioned. It is important to note, consistent with the point made by C\&L, that children do not accept ready-made the units as organized by caregivers, but rather, that children's linguistic usage of desire constructions is indeed sensitive to the language patternings available in their caregivers' discourse. Rather than emphasizing that caregivers or children talk about desire, the notion of indexicality contributes by providing a suggestion for how children can grab onto culturally relevant aspects of an understanding of mind by attending to the patterned uses of particular linguistic constructions (see Budwig 2003).

Around the turn of the twentieth century, the study of human development often included close discussion between psychologists, linguists, and anthropologists. C\&L's article brings us back to such a time and encourages developmental psychologists to not 
be limited by current blinders in how to conceive of the relationship between language and thought.

In this commentary I have suggested two ways in which their argument can be strengthened, namely, by moving beyond an analysis of words and by picking up on the notion of indexicality. Grounding discussion of children's construction of mind in the interdisciplinary study of language, thought, and culture will ultimately provide fruitful new avenues for future analyses.

\section{Agency mediation and an understanding of the mind}

\section{Olga Chesnokova \\ Department of Psychology, Moscow State University, Moscow 103 009, Russia.olches@olches.ps.msu.su}

Abstract: This commentary is an attempt to give a Vygotskian perspective on Carpendale's \& Lewis's (C\&L's) target article. The article uses ideas that are well familiar to Vygotsky's scholars. However, it develops these ideas further and raises important empirical questions about the role of social interaction in the development of social cognition. The article provides a fresh view on the old problems and frames themes traditional for the English-speaking developmental psychology into a broader international perspective.

Paradoxically, Carpendale \& Lewis's (C\&L's) article is probably the first serious attempt to frame the discussion on the development of social understanding in a social context. The main point of C\&L is close to the one targeted by Lev Vygotsky, who raises the question of whether children create their theories of the social world on the basis of their innate modules and independent life experiences or they create those theories within a social interaction (1999). Having reviewed a vast number of sources, the authors come to the conclusion that social understanding is "the emergent product of social interaction" (Gibbs 2001). Within a Vygotskian social-constructivist perspective, the idea seems quite mundane. However, though implanted in the context of ongoing discussions about innate "modules," "implicit knowledge," and inherent understanding of "affordances" - concepts that have long dominated developmental psychology in English-speaking nations - the ideas proposed in the target article are actually fresher than they might seem at first glance.

Having discussed the ample empirical evidence demonstrating that a higher level of social understanding is more evident in children who have closer positive relationships with significant others than in those who have less close relationships, C\&L use this evidence to outline directions for future research that could bring this relatively stale area of studies out of the deadlock of the "theories of mind." One of these directions might be studying multiple nonverbal interactions that occur in the natural social setting and play a major role in promoting social understanding, especially at the early, prelanguage stages of development. The central claim of C\&L is that the focus of research should be shifted from investigating mental states and mentalist concepts as the outcomes of children's understanding of others' minds to the careful analysis of social interactions and cultural contexts that shape the content of children's social knowledge. In fact, the authors resurrect the old but fundamental question of how social interaction should be understood. Is social interaction "based on the interaction of minds which can be properly understood only when one takes into account what people think about other people's thoughts" (Perner \& Wimmer 1985, p. 438), or is the mind itself "the product of social life and ... activity which was earlier shared by two people" (Luria 1969, p. 143)? I am sympathetic with the authors' adoption of Chapman's (and Luria's) view on this point: that social interaction is at the base of mental and social understanding.

But do social interactions directly shape children's growing un- derstanding of mental states? Or is there a mechanism that mediates the link between social interactions and children's social understanding? Closely following Wittgenstein's view of the "socially projected" nature of mental activity, the authors at the same time strongly emphasize that a child is an active agent within social interaction, and not a passive recipient of socially induced knowledge. In this, C\&L closely approach the "activity principle" in the understanding of cognitive development that has long been employed by the Vygotskian tradition (see El'konin 1969). The "activity principle" suggests that the simple fact of a child's involvement in social interaction is a necessary but not a sufficient condition of the child's improvement of his or her social understanding (as well as his or her understanding of other people's mental states). It is important to analyze what position (active or passive) the child, as an agent, takes in this social interaction.

One striking example of the role of agency in social interaction can be found in studies on children's moral understanding. In one of these studies, preschool children who had previously transgressed on moral tasks (such as deliberate cheating) were asked to instruct other transgressors to observe the same moral rules (Subbotsky 1993). Although in the post-tests all the "instructors" revealed an equal degree of understanding of what was right or wrong to do in social interactions with a "moral underpinning," only those children who had actively accepted the position of a moral instructor did not repeatedly transgress if given a chance to do so. This example empirically illustrates a circularity puzzle (a circle) that is inherent in the claim that children are agents within a social interaction: that is, whereas interactions can facilitate children's understanding of other people's (and their own) mental states, it is the child him- or herself who ascribes value to the acquired knowledge. On this ground, the claim promoted by C\&L, that children's social understanding is shaped while they interact with others, should be supplemented by the principle of "agency mediation." This means that social interaction can elicit a proper (and not only formal or verbal) social understanding in a child only when the child takes an active position within the interaction and implants the acquired social knowledge with personal (and emotional) meaning. In fact, this provides an answer to the question formulated by Astington and Olson (1995): Does a child passively adopt mentalist concepts available from cultural social surroundings, or does the child actively construct these concepts within the interactions?

The principle of "agency mediation" also gives us a chance to integrate a vast body of fascinating studies of the social context of problem-solving interactions and "theory of mind" into the studies on social intelligence and personality (Cantor \& Kihlstrom 1989; Dunbar 1996; Garton 2004; Gauvain 2001). The context of an individual's social life shapes his or her understanding of mentalist states (such as beliefs, desires, intentions, attitudes) only when this context is filtered through the individual's integrated "self" (personal identity). The "self" (agent) exercises control over the person's immediate actions and also sets up the individual's ultimate life goals. In light of the "agency mediation" principle, we can now reformulate the final "developmental goal" of social cognition and social understanding: to achieve an understanding of other people's minds for the purpose of using this knowledge for building stable and happy personal (moral, social, business, and other) relationships.

To conclude, C\&L's paper utilizes ideas that have been around for a considerable period of time. Nevertheless, the paper does raise interesting and intellectually challenging theoretical and empirical questions about the role of social interaction for the development of social cognition. It stimulates a refreshing way of thinking and gives a good stir to the problem that has been boiling for a long time within the encapsulated circle of English-speaking developmental psychology. 


\section{I ain't got no body: Developmental psychology must be embodied and enactive, as well as "social"}

\author{
A. P. Craig a and L. Barrett ${ }^{b}$ \\ a University of Cape Town, Commerce Faculty, Management Studies, \\ Rondebosch 7700, Cape Town, South Africa; b School of Biological Sciences, \\ University of Liverpool, Liverpool L69 7ZB, United Kingdom. \\ biblis@iafrica.com L.Barrett@liverpool.ac.uk
}

\begin{abstract}
Although we agree with the authors' criticism of the reigning approach to children's sociocognitive development, we raise three further issues. First, "mind talk" is not, in fact, any different from the other aspects of the social world about which children learn. Second, there is no choice between either the "single mind" or the "social context." Finally, there is a spurious separation between organism and environment.
\end{abstract}

In this target article, the authors take issue with the reigning "individualistic" approach in theories of children's development of social understanding. In what follows, we want to support their criticism of this approach, but in somewhat different terms. We therefore raise three questions for the authors to consider.

1. What if we were "Antipodeans"? Richard Rorty's (1980) interesting thought experiment regarding persons without minds, the Antipodeans, has something to teach us as far as "constructing an understanding of mind" goes. Among these people, according to Rorty, "neurology and biochemistry had been the first disciplines in which technological breakthroughs had been achieved, and a large part of the conversation of these people concerned the state of their nerves." Also, given the state of their knowledge of physiology, "each well-formed sentence in the language which anybody bothered to form could easily be correlated with a readily identifiable neural state" (p. 71). Using this device, Rorty wanted to show that nothing "hangs on the distinction between mind and body," that the "mind" is not something about which we have to have knowledge, and that "No predictive or explanatory or descriptive power would be lost if we had spoken Antipodean all our lives" (p. 120). We introduce Rorty's story here to undermine any attachment to learning mind-talk as special, as somehow requiring answers different in kind (i.e., more focused on the inner experiences or the "free mind" of the developing organism) from those answers dealing with the norms, habits, rituals, beliefs, and practices of the social group to which the child belongs. In these terms, therefore, we want to rule out "theories of mind" on two counts: (1) as theories of anything, and (2) as theories (for the latter, cf. Millikan 1995, pp. 51-82).

In addition, we want to cast aside "simulation theory" and especially its focus on "introspection," and propose that children are most likely to be genetically endowed with strong pattern recognition abilities and the tendency to establish correlations between, in "our" case, mind talk and various associated behaviours, first in others and later in themselves, after the onset of language. This follows from Millikan's (1995) views that folk psychology merely describes "the competence" of "certain devices inside us" and that it is properly the job of the neuroscientist to look for the devices that show particular kinds of competence. Concerning ourselves thus with "certain devices inside us" places our position alongside those favouring attention to our genetic endowments regarding learning.

2. Does gradual learning necessarily imply constructivism? The fact that learning is gradual does not seem to favour one or another theory on the relationship between "the social" and the tools or resources with which the child becomes proficient. That is, the connective changes made during learning and the synaptic adjustments the brain undergoes (as change is steered by both one's genetic heritage and culturally specific, unique experiences), all take time (cf. Churchland 1996). Phrased differently, gradual change alone does not privilege a more social or constructive focus on things.

In order to support their view, the authors must do more than provide evidence to show that social history shapes individual differences in time and space. In other words, what remains empir- ically open has to do with the mechanisms of transmission between social history and individual knowledge acquisition specifically, how this transmission occurs from case to case or task to task (through internalisation, in Vygotsky's terms, or gradual construction, in the terms of the authors, or the twin mechanisms of assimilation and accommodation, in Piaget's model of knowledge development).

The only thing that seems certain is that one's knowledge of the world, or "that small part of it that is relevant to one's own practical concerns . . . is embodied in the peculiar configuration of one's 1014 individual synaptic connections ... which are set to progressively more useful values during learning and development" (Churchland 1996, p. 5), and that these configurations are the result of a "merging" of organism and environment (in Millikan's [1995] terms); there is therefore no choice between "the single mind" and the "social context," contrary to Raver and Leadbeater's (1993) assertion; it is a bewitchment precisely of the kind that Wittengenstein warned against.

3. Is there any such thing as a "single mind"? To expand on this point, it seems to us that, once we move away from such misleading distinctions, labels ("theory-of-mind"), and other bewitchments and from our tendency to utter inarticulate sounds (another Wittgensteinian jab at bad thinking), we are probably in a better position to come up with answers to why-questions; for example, why "aspects of social interaction are correlated with social understanding." As Millikan (1995) states: "There is no clear line but only the most arbitrary demarcation between the organism considered as process and its environment. The organismic process has no skin.” (pp. 179-80). Hence merely adding - in some or other old or new configuration - "the social," as the authors suggest, does not clarify the relationships between learning and maturity/development, nor its other or related side, organism and environment.

What has to be worked out are the modes of connection between these processes and factors, and these probably differ from case to case. That is, we suspect that the gap between, for example, "initial knowledge" in the domains of "physics, psychology, number, and geometry" (cf. Spelke 1994, p. 433) and "our mature knowledge," which, as Spelke tell us, "does not appear to be shared by infants" (p. 435), will be filled in through the bits and pieces of time- and place-appropriate features which are mediated to the developing organism by its caregiver(s) (cf. Feuerstein's description of “mediated learning experience" [Feuerstein 1980, p. 15], which is a clinical elaboration of Vygotsky's Zone of Proximal Development [Vygotsky 1978, p. 86]). It is these changes over time, whereby "initial" infant knowledge is displaced, revised, and overturned on its route to becoming adult knowledge, that seem to us to offer empirical possibilities for examining the interaction between development and learning and organisms and environment. That a refocus on embedded cognition occasions methodological adjustments is also recognised (cf. Craig 2003). Thus, once we slough off any lingering attachment to an isolated "mind," our theories of learning will automatically bring brain into world (and vice versa) and will consider cognition in context (Clark 1999; Hutchins 1995; Rowlands 1999).

\section{Children, chimpanzees, and social understanding: Inter- or intra-specific?}

\author{
Timothy J. Eddy \\ Psychology Department, Salem State College, Salem, MA 01945. \\ timothy.eddy@salemstate.edu
}

Abstract: Theories of children's understanding of mind benefit from rigorous interpretations of demonstrations of similar understandings in closely related species. This commentary describes how Carpendale \& Lewis's (C\&L's) argument could be made more persuasive with a more rigorous interpretation of the studies of chimpanzees' understanding of mind. 
Carpendale \& Lewis (C\&L) provide a long-overdue treatment of the development of social understanding as influenced by social interactions. As such, their target article goes a long way in reconciling the ideas of Wittgenstein, Piaget, and Vygotsky. When removed from evolutionary considerations, C\&L's arguments might lose considerable appeal. In an effort to place their work in a larger evolutionary context, and in the context of what the authors call the "lean interpretation" of joint attention, they cite some work suggesting that chimpanzees "do not appear to understand the psychological significance of gaze direction" (sect. 3.1, para. 8). Curiously, considering the authors' thesis that social interaction plays a major role in constructing understandings of mind, they fail to discuss the social matrix of the chimpanzees so referenced.

Povinelli and Eddy (1996) carried out a series of studies that appear very similar to the ones that C\&L cite from Povinelli (1999). That is, the 1996 work included findings that, to quote C\&L (sect. 3.1, para. 8),

chimpanzees trained to beg with a hand-out gesture and given the opportunity to beg for food from one of two experimenters sitting side-byside are initially just as likely to beg from someone with her eyes closed, with her head turned away, or even with her head covered by a bucket as they are to beg from someone with her eyes open.

These are some of the exact findings of Povinelli and Eddy (1996). Unfortunately, I have been unable to obtain reprints of the Povinelli (1999) report from his lab, so it is unclear whether he has replicated nearly our entire series of experiments (14 studies with chimpanzees and one with young children) or whether C\&L are actually referring to Povinelli and Eddy (1996). Regardless of the answer to that question, the 1996 report brings to the fore an additional interpretation of the chimpanzee findings distinct from C\&L's "lean interpretation," which has a direct bearing on one of the central ideas they raise.

Specifically, in the 1996 work, one of the interpretations of the chimpanzees' failure to take into the account the visual perception of others concerned the interspecific nature of the tasks. That is, the chimpanzees in those studies were required to reason about the visual perception of members of a different species (human experimenters). According to this interpretation, the chimpanzees in these studies might in fact show very different results if required to reason about the visual perception of other chimpanzees (rather than humans).

Because these studies have not actually been carried out, it is unclear whether the failure of the chimpanzees under these circumstances is because they lack the capacity to make attributions of visual perception to others (C\&L's "lean interpretation") or because they have simply been asked to make attributions to member(s) of the wrong species. Given the human tendency to attribute mental states to some (but not all) animals (see Eddy et al. 1993), this becomes a distinct possibility when applied to chimpanzees.

Where would an acceptance of the "chimpanzees' failure due to the interspecific nature of the tasks" interpretation leave C\&L's ideas concerning the development of children's social understanding? Probably not in a fundamentally different place from where it is now. Having said that, if explicit linkages between the development of differing (or similar) abilities in members of different species can be made with data that have singular (rather than multiple) interpretations, then such ideas could be placed squarely and firmly in our growing understanding of the relation between psychological phenomenon and evolutionary facts.

\section{ACKNOWLEDGMENTS}

I thank David Gow for his information, support, encouragement, good humor, and worthiness as a colleague; and thanks to Alessandra Bianchi for proofreading at the eleventh hour.

\section{More than a context for learning? The epistemic triangle and the dialogic mind}

\section{Charles Fernyhough}

Department of Psychology, University of Durham, Science Laboratories, Durham DH1 3LE, United Kingdom. c.p.fernyhough@durham.ac.uk

http://www.dur.ac.uk/c.p.fernyhough

Abstract: Theory-of-mind (TOM) competence is under-specified in Carpendale \& Lewis's (C\&L's) account. In the neo-Vygotskian alternative outlined below, TOM development is driven by the internalisation of dialogic exchanges which preserve the triadic intentional relations of interactions within the epistemic triangle. On this view, TOM competence stems from children's ability to operate flexibly with the multiple perspectives manifested in internalised dialogue.

Carpendale \& Lewis (C\&L) pull together an impressive range of findings on social-environmental influences on theory-of-mind (TOM) development, and set out a welcome new theoretical framework for understanding how such capacities might emerge within interpersonal interaction. Where their article is less satisfying is in specifying those cognitive mechanisms that might result from such developmental processes. That is, the authors give an interesting and plausible account of how TOM competence develops, but they never really say what it $i$. In particular, the authors are unclear about whether the epistemic triangle serves primarily as a context for learning the criteria for the correct application of mental state terms, or whether it goes on to play a role in children's subsequent solo reasoning about the mind. The authors hint that they have the latter in mind when they note that children's facility with mental state language, developing hand-inhand with their understanding of the social contexts within which such language use is appropriate, allows them to become "able to think about situations abstracted from their practical context of interaction and ... to anticipate and reason about situations such as the false belief task" (target article, sect. 4.1, para. 9). At the same time, this account leaves it unclear whether the end product of development, namely TOM competence, amounts to much more than the correct application of mental state terms and concepts in accordance with externally derived criteria for their use. In this case, an unsatisfactorily individualistic account of TOM competence appears to have slipped in through the back door, and one is left wondering what the C\&L account can offer that the conventional theory-theory cannot.

It is here that the authors could have gone further with their interest in the work of Vygotsky. Although Vygotsky did not directly address the problem of TOM development as it has been characterised by researchers in this area, several aspects of his writings are potentially relevant here. First, Vygotskian ideas can be useful in an enculturation account of the sort that C\&L discuss in section 2.1. Second, the idea of naïve participation, which sees infants being drawn into practices that they will only later come to understand (Vygotsky 1934/1987, Ch. 4), is central to C\&L's conception of how an "initial, fragile social understanding" (sect. 4.1, para. 9) can become transformed into a fully fledged TOM. Third, Vygotsky's concept of the zone of proximal development (Vygotsky 1934/ 1987, Ch. 6) provides a framework for understanding how sensitive input from caregivers can "scaffold" children's acquisition of mental state terms and concepts (Meins et al. 2002). Fourth, Vygotsky's idea that language can be used as a psychological tool (Vygotsky 1930/1978) in augmenting pre-existing cognitive capacities suggests that private and inner speech may play important roles in mediating children's own reflections on the nature of mental states and processes (Meins et al. 2003). Finally, the Vygotskian notion of internalisation of semiotically mediated interpersonal activity (Vygotsky 1931/1997) may prove useful for our theorising about how children's developing TOM abilities are rooted in their experience of social exchanges.

I suggest that it is this latter aspect of Vygotsky's theory that can make the difference between an essentially individualistic account of TOM development based primarily on the learning of terms for 
mental state concepts, and an account that sees social understanding as genuinely and profoundly rooted in social experience. In a now-familiar set of arguments, Vygotsky proposed that higher mental functions such as mediated memory and self-regulatory inner speech are formed through the progressive internalisation and transformation of semiotically mediated interpersonal exchanges (Vygotsky 1934/1987, Ch. 4). In internalising dialogue with others, the child internally reconstructs the dynamics of that dialogue with, crucially, all of its triadic intentional relations intact. The essential thing about dialogue, for our purposes, is that it involves a simultaneous unity of different perspectives (Holquist 1990) on the element of reality to which the dialogue is intentionally related. The internalisation of dialogue, for which normal children are pre-linguistically prepared by the socio-affective responsivities and joint attentional capacities described in section 3.1, therefore involves children taking on a semiotic (usually natural-language) representation of the alternative perspective manifested in that dialogue (Fernyhough 1996; 1997).

It is this permeation of children's thinking by the different perspectives encountered within the epistemic triangle that forms the basis of their developing ability to operate with these perspectives, first in naturalistic social-cognitive reasoning contexts, and later in more formalised tests of TOM. The end result of internalisation is thus an ongoing interplay between different, simultaneously held perspectives on reality, represented first in private speech and ultimately in dialogic inner speech. To the extent that it requires the flexible and simultaneous co-ordination of multiple perspectives on an aspect of a task situation - such as the location of an unexpectedly transferred object or the phenomenal qualities of an item in the appearance-reality task (Fernyhough 1996) - TOM reasoning represents the pre-eminent example of dialogic thinking.

Allowing the epistemic triangle to become internalised would therefore provide $C \& L$ with a specific cognitive mechanism through which children could operate with the mental states of others and "coordinat[e] these often differing perspectives with their own beliefs" (sect. 4.2, para. 1). It would also provide a framework for making sense of the social-influences findings that went beyond the (admittedly important) issue of learning the correct use of mental state terms. For example, by focusing on how caregivers differ in their ability to construct dialogues with their children in which the intentional stance is taken (Garfield et al. 2001), the neo-Vygotskian approach is better placed to account for the evidence that very early differences in maternal mind-mindedness relate to individual differences in TOM several years later (Meins et al. 2002; 2003). Much more than a mere context for learning, the internalised epistemic triangle becomes an essential part of the child's solution to the moment-by-moment challenge of engaging with other minds.

\section{The roots of social understanding in the attachment relationship: An elaboration on the constructionist theory}

\section{Peter Fonagy \\ Sub-Department of Clinical Health Psychology, University College London, London WC1E 6BT, United Kingdom. p.fonagy@ucl.ac.uk http://www.psychol.ucl.ac.uk/psychoanalysis/peter.htm}

\begin{abstract}
It is argued that constructionist theory provides only a partial account of how secure attachment leads to better social understanding. In addition to cooperative parent-child relations, the more efficient arousal and affect regulation system of secure infants, and developmental moderators of the processes of imitation, may play a part in explaining the association and offer clues as to how effective social understanding is generally acquired.
\end{abstract}

Carpendale \& Lewis (C\&L) make a strong case that past attempts at accounting for the influence of social interaction on the devel- opment of children's social understanding ${ }^{1}$ within the confines of the three prominent models of theory of mind acquisition have been at best partial. They reject both the enculturation and the extant developmental propositions (maturation, theory-theory, and simulation) in favour of a model that assumes that social understanding is an emergent property of the child's experience of certain regularities in interaction with others. The "epistemic triangle" (i.e., referential interactions between infant and caregiver about the object) is assumed inevitably to generate the discovery that others sometimes have different beliefs about the world from one's own. If we, taking the point of view of the infant, assume the existence of a stable external world, the actions of others in communicative interactions can only be understood given the supposition that they have different beliefs about aspects of the world. Children achieve comparable levels of development at similar ages simply because of the commonalities of their experience. The corollary of this is that differences in the acquisition of social understanding are to be understood in terms of crucial differences in their experience of triadic interactions. For example, our results from the London Parent-Child Project (Fonagy et al. 1997) that children securely attached at one year were significantly more likely to pass the Belief-Desire reasoning test at five and a half years $(n=90, p<.005$, OR $=3.8$, CI: $1.5-9.9$ ) may be understood in terms of attachment being a good marker for a cooperative parent-child relationship, which, according to constructivist theory, is the ideal context for the development of knowledge.

Although I do not fundamentally disagree with either the spirit or the details of the case advanced in the target article, there are important gaps in the model advanced by the authors. The target article is helpful in drawing attention to the common ground between Bowlby's (1980) and Piaget's (1945/1962) thinking, particularly in relation to Piaget's notion of schemes and Bowlby's assumptions concerning internal working models. However, how do secure-autonomous internal working models in the parent lead to the kinds of cooperative relationships "that might permit unrestrained communication, that allows participants to understand each other fully" (Habermas 1983/1990)? We have to assume a dialectic model of self-development (Hegel 1807) where the child's capacity to create a coherent image of mind is critically dependent on an experience of being clearly perceived as a mind by the attachment figure. The now overwhelming evidence for the transgenerational transmission of attachment security is consistent with this (van IJzendoorn 1995), and this may link to the emergence of mentalisation in the child (for a review, see Fonagy et al. 2002). Accumulating transgenerational evidence suggests that mothers who conceive of their own childhoods largely in mental state terms are more likely to go on to develop secure attachments with their children (Fonagy et al. 1991; Target et al., in press) and to have a more differentiated mentalised picture of their infant (Muzik \& Rosenblum 2003; Slade et al. 2001), which in turn is associated with more mind-minded comments (Muzik \& Rosenblum 2003) and infant security (Slade et al. 2001). As the work of Elizabeth Meins and colleagues (2001) suggests, mothers whose state of mind with respect to attachment may be described as secure (Main 2000) expose the infant to more mental state language, which in turn advances the child's social understanding.

Yet, more than mental state language, the coherence with which the child's mental state is perceived may be the critical variable. In a study by David Oppenheim (Koren-Karie et al. 2002; Oppenheim \& Koren-Karie 2002), mothers were asked to narrate a videotaped playful interaction that they had just had with their infant. Mothers who were reflective in their narratives, able to see various experiences through the child's eyes and gain new insights as they talked, were far more likely to have securely attached infants than mothers who either had preset conceptions of the child which they appeared to impose, or disengaged from trying to understand what was on the child's mind. Most pertinent was the observation that disorganised attachment classification was associated with mothers who were incoherent, switching between the above categories and not fitting well into any of them. Thus, 
"mind-mindedness" and security of attachment in the caregiver appear to go together and are associated with a coherent working model of the child that is richly imbued with representations of internal states. This is the substance of the collaboration and cooperativeness at the heart of constructionist theory.

Cooperative relationship rooted in the coherence of the perception of the child's mental state may not be the only factor. The development of the key psychological capacities that underpin theory of mind may be grounded in the attachment relationship (Fonagy \& Target 2002; Fonagy et al. 2002). It is quite probable that an important mediator of the association of secure attachment and theory-of-mind development lies in the regulation of physiological arousal. Secure attachment may be conducive to mentalising because it facilitates an optimal level of arousal (Field 1985; Kraemer 1999; Panksepp et al. 1999). Mentalising depends substantially on optimal prefrontal cortex functioning (Adolphs 2003; Blair \& Cipolotti 2000; Rowe et al. 2001; Siegal \& Varley 2002; Stuss et al. 2001). Medial and orbital prefrontal cortices have been linked to the regulation of interpersonal relationships, social cooperativity, moral behaviour, and social aggression (Damasio 2003; Davidson et al. 2000; Greene \& Haidt 2002; Kelley et al. 2002; Schore 2003). The optimal functioning of the prefrontal cortex in turn depends on optimal arousal. Neurochemical regulation of the prefrontal cortex is complementary to that of posterior cortex and subcortical structures ${ }^{2}$ (Arnsten 1998; Arnsten et al. 1999). Arnsten and Mayes (Arnsten 1998; Arnsten et al. 1999; Mayes 2000) have argued that when arousal exceeds a certain threshold, it is as if a neurochemical switch is thrown. This switch shifts us out of the executive mode of flexible reflective responding into the fight-or-flight mode of action-centred responding. Those with insecure or disorganised attachment relationships are sensitised to intimate interpersonal encounters and experience higher arousal, and the relative level of arousal in the frontal or posterior part of the cortex readily shifts posteriorly.

Similar arguments could be mounted in relation to effortful control. The capacity to inhibit a dominant response in place of a subdominant one is a key achievement of early development (Kochanska et al. 2000; Rothbart et al. 2000). It also appears to be powerfully predicted by security of attachment at one year (Kochanska 2001; Kochanska et al. 2000; Kreppner et al. 2001). Mentalising involves setting aside immediate physical reality in favour of a less compelling reality of the other's internal state. Previous studies have linked the acquisition of effortful control to performance on the false belief tasks as they follow a common developmental timetable and share a common brain region and yield common types of pathology (Carlson \& Moses 2001). Hence, we would argue that children with a background of secure attachment are more rapid in their acquisition of mentalisation in the context of social relationships because secure attachment has equipped them with the capacity appropriately to attend selectively to critical aspects of such interactions.

More recently one of the most creative writers in this field, George Gergely (2003), has identified two strategies of imitation in 14- to 18-month-old infants; 14-month-olds use an observational learning strategy based on an active process of evaluation of situational constraints using the principle of rational action (Gergely \& Csibra 1997; 2003), imitating only when the other's action is not explained by situational constraints and therefore points to the affordance property of the object (Gergely et al. 2002). (In a modified replication of Meltzoff's famous study of the infant imitating the experimenter switching a light on in a box by touching it with his head, Gergely et al. [2002] showed that imitation does not occur when the hands of the actor are visibly constrained, and when the infant interprets the use of the head as a rational action justified by the actor's immobilisation and not relevant to the infant because the infant's hands are free.) Four months later, the physical constraints appear irrelevant (Gergely 2003), as the infant imitates regardless of the observed physical constraints; it is assumed that this is because the infant presumes a cooperative intention to teach on the part of the actor. Thus, by 18 months the infant presumes that the actor as a rational mental agent has already tested the rationality of the novel action him- or herself.

Gergely's work has an important qualification for constructivist theory insofar as that the gradual emergence of mentalisation appears to change the experience of the "epistemic triangle" for the infant. The experience of interchange evolves from a teleological experience at 14 months to the beginning of a mentalistic one by 18 months. ${ }^{3}$ Gergely (2003) argues that one of the central environmental factors that contributes to the shift from teleological emulation to the assumption of a cooperative intention to teach, is previous experience of "benevolent teaching" interactions initiated by the caregiver. It is likely that in dysfunctional attachment contexts, the infant learns to interpret parental initiation of eye contact and other communicative attention-directing behaviours as cues signalling that potentially harmful interactions are likely to follow. The infant may develop the defensive coping strategy of inhibiting the mentalistic interpretation of such communicative behavioural cues. Hence, in severely dysfunctional attachment contexts, infants are likely to fail to interpret mentalistically the communicative referential cues of the demonstrator as indicating a benevolent and cooperative intention to teach.

In brief, while broadly accepting the constructivist model, we suggest (1) that specific capacities (arousal regulation, effortful control) link the secure base that generates secure attachment with evolving symbolic function; (2) that the link of the secure base phenomenon to the development of mentalisation will be increasingly understood to be causal rather than correlational, in that the group of capacities that underpin adequate social understanding (what Bogdan [1997] called interpretation) are evolutionarily tied to it; in other words, that the evolutionary function of the attachment relationship in humans goes beyond the protection of the vulnerable infant to providing an environment within which social understanding may be acquired; and therefore, finally (3) that deficits in attachment create a vulnerability in the child to later environmental challenges.

\section{NOTES}

1. Our preferred term for social understanding has been "mentalisation" (see Morton 1989).

2. As the level of cortical activation increases through mutually interactive norepinephrine alpha 2 and dopamine D1 systems, prefrontal cortical function improves on capacities such as anticipation (shifting of attention), planning/organisation, and working memory. With excessive stimulation, norepinephrine alpha 1 and dopamine D1 inhibitory activity increases; the prefrontal cortex goes "off-line," and posterior cortical and subcortical functions (e.g., more automatic functions) take over. Increasing levels of norepinephrine and dopamine interact such that above threshold, the balance shifts from prefrontal executive functioning to amygdala-mediated memory encoding and posterior-subcortical automatic responding (fight-flight-freeze).

3. In this sense, of course, the constructivist account skirts circularity but gets round it by the assumption of the gradual emergence of the mentalising capacity.

\section{No (social) construction without (meta-)representation: Modular mechanisms as a basis for the capacity to acquire an understanding of mind}

\author{
Tim P. German a and Alan M. Leslie
}

a Department of Psychology, University of California, Santa Barbara, CA 93106-9660; benter for Cognitive Science, Rutgers University, Piscataway, NJ 08854. german@psych.ucsb.edu aleslie@ruccs.rutgers.edu

Abstract: Theories that propose a modular basis for developing a "theory of mind" have no problem accommodating social interaction or social environment factors into either the learning process, or into the genotypes underlying the growth of the neurocognitive modules. Instead, they can offer models which constrain and hence explain the mechanisms through which variations in social interaction affect development. Cognitive mod- 
els of both competence and performance are critical to evaluating the basis of correlations between variations in social interaction and performance on mental state reasoning tasks.

Carpendale \& Lewis (C\&L) offer a theory of the acquisition of an understanding of mind where varieties in the structure, amount, and types of social interaction play a pivotal role. Part of their argument is that other theoretical accounts underestimate, and indeed are inconsistent with, evidence for correlations between indexes of social interaction and the development of an understanding of mind. The authors single out theories that posit an innate modular basis to the capacity to acquire a "theory of mind," saying that such theories "[live] uneasily with the accumulating evidence, such as the sibling effect ...., showing that the nature of the social interaction children experience is closely related to the development of their social understanding" (target article, sect. 2.2 , para. 5 ).

Though common, the authors' view misrepresents the modular theory as claiming that "the solution to this problem of understanding the mind" is somehow "innately given" (sect. 2.2, para. $5)$. The authors even contrast the modular view with the view that the capacity to acquire an understanding of mind is innate. But it is precisely this latter claim that is endorsed in the modular framework: "theory of mind" is described as having an innate and possibly modular basis, not as consisting in its entirety as either innate or as a module (Leslie 2000; Scholl \& Leslie 1999; 2001). The modular view is also commonly accused - and the target article is no exception - of being inconsistent with demonstrations that there is "development" in the domain of understanding mind. Yet, roles for both known developmental mechanisms, namely, maturation and learning, are described in the modular theory that the authors attack: Maturation of hardwired mechanisms enables the child to make appropriate sense of social inputs and hence to learn about the social and mental worlds (see German \& Leslie 2000).

In a section on future research directions, the authors bemoan the imprecision of what the term "understanding" might mean in this domain (sect. 5, para. 6). Here we agree, and we draw their attention to the fact that in the modular approach they reject, intuitions about what should constitute having or not having "understanding" have been replaced by attempts to specify underlying cognitive mechanisms, to delineate what their inputs are, to characterize the representations they deploy, and to study how they interact with domain-general processing mechanisms in solving "theory of mind" problems (e.g., German \& Leslie 2001; Leslie 1987; 2000; Leslie \& Polizzi 1998).

Throughout this same section, the authors themselves call for research to "move from the intriguing findings relating individual differences in the social experiences of infants and their social understanding to research that explores the processes involved" (sect. 5, para. 4). But, despite a laudable insistence on the importance of models of process, they offer not a single proposal about how "construction of understanding of mental states from social interaction" is supposed to work. Instead, it is left to "future research" to answer questions such as: "how . . . mothers' tendency to talk about their infants in psychological terms is positively associated with their child's understanding of false beliefs almost four years later?" (sect. 5, para. 4).

What is instructive about these gaps is that the modular framework, far from "living uneasily" with the importance of social input, is the only approach that has concrete proposals about how social factors play their role. As Roth and Leslie (1998) put it, "the emergence of [the "theory of mind" mechanism] solves a critical but limited problem. It allows the young brain to attend to ... mental states despite the fact that such states cannot be seen, heard, felt, or otherwise sensed ... [and] being able to attend to mental states is a prerequisite for learning about those states" (p. 27; emphasis in original). The ability to attend to mental state properties, such as pretending and believing, is a critical part of what makes the social world the world it is and a critical part of what makes experience of that world into social experience. With- out this ability, though he or she may be constantly surrounded by playful laughter, a child develops in social isolation; such is the lot of the child with autism. Beyond this basic ability lies the later success in answering questions about false beliefs. Here again, the modular theory has made concrete proposals about how the child learns about these social situations, learning things like "beliefs tend to be false under systematic circumstances, surprise occurs when a false belief is relieved, false beliefs can be induced in opponents ... [and] how to achieve the latter and get away with it."(Roth \& Leslie 1998, p. 28).

C\&L rightly remind us of the danger of "neurotic task fixation" (sect. 4.1, para. 4; see Bloom \& German 2000), and rightly acknowledge that meta-analysis cannot resolve the question of whether there is conceptual change (see Scholl \& Leslie 2001). Yet they make the very mistakes they warn against by implicitly assuming that "understanding" the concept of belief is just the same thing as passing a false belief task. In particular, the authors fail to distinguish competence and performance in their interpretation of the false belief task, and, hence, in their interpretation of its correlation with indexes of social interaction.

Positive associations between indexes of social interaction (number of siblings, security of attachment, maternal mind-mindedness, parenting style, etc.) and mental state "understanding" may be the result of many different factors in many different mixes. Plausibly, some of these factors reflect shared genotypes, some the structure and richness of inputs to relevant learning mechanisms, and of course, let us not forget good old-fashioned practice - in this case, practice with social cognizing. The studies reviewed by the authors under this heading have invariably demonstrated correlations with performance on a false belief task. There is already good evidence for a strong heritable component to false belief performance (Hughes \& Cutting 1999). There is also mounting evidence suggesting that an important, and perhaps critical, factor in performance on false belief reflects inhibitory processes that develop across the early years (Carlson et al. 1998). Curiously, there is also at least one demonstration of a sibling effect on executive tasks without replication of the sibling effect on false belief (Cole \& Mitchell 2000). It is even possible that correlations between various social indexes and "theory of mind" are mediated by domain-general processes.

In conclusion, it is only by developing models of successful performance on various component "theory of mind" skills, such as recognition of agency in infancy (e.g., Johnson 2003), pretend play (Leslie 1987; 1994), belief-desire reasoning (Leslie \& Polizzi 1998), and recall of the contents of one's own past mental states (Barreau \& Morton 1999), and by studying their specific neurocognitive basis (Frith \& Frith 1999; Gallagher \& Frith 2003) that we can hope to understand how the brain has been organized to acquire this important knowledge base.

\section{Individualism and cognitive development}

\author{
Philip Gerrans \\ Department of Philosophy, University of Adelaide, Adelaide, SA 5005, \\ Australia.philip.gerrans@adelaide.edu.au \\ http://www.adelaide.edu.au/philosophy/people/pgerrans/
}

\begin{abstract}
Individualism is not inconsistent with social interaction; it is required to explain it. Social exchanges, evidenced in gaze monitoring, social referencing, emotional responses, protodeclarative and imperative pointing, pretence, play, and conversation all play a role in development, but the nature of that role is opaque without an understanding of the cognitive mechanisms on which they depend.
\end{abstract}

The normal child is confronted not by an intentionally inert world of moving objects but by one of animate bodies, facial expressions, gestures, vocalizations, and emotions. In order to cognise the world in this way, the child does not need to possess a fully fledged 
intentional psychology; rather, she possesses a set of cognitive mechanisms whose proper function is to sensitise her to possibilities for intentional engagement with her surroundings. As a result of this engagement, the normal child will develop the concepts of intentional psychology.

I take it Carpendale \& Lewis (C\&L) would agree with this characterisation; however, their approach does not lead to any deeper understanding of either the mechanisms which initially sensitise the child to her social environment, or those which subsequently lead to the type of conceptual understanding tested in false belief and other "theory of mind" (TOM) tasks. There are two reasons. First, they endorse Wittgenstein's argument that cognitive competence is mastery of a practice best understood as a skill rather than an intellectual or theoretical achievement. Second, the authors do not connect their account of social understanding to the understanding of autism. The two issues are related.

Wittgenstein gives no explanation of the way social interaction produces social understanding. This is because he regards it as an essentially unanalysable skill which, once acquired, allows the subject to perceive mental states directly. His reasons for rejecting intellectualist accounts of skill acquisition (fast, fluent, and flexible responses cannot be governed by rules understood as algorithmic reductions of theoretical inferences) combine with his conceptual analysis of the concept of an intentional state. The folk concept, properly analysed, of an intentional state is not that of a covert cause of overt behaviour. Social understanding feels like immediate and non-theoretical recognition of mental states exhibited in overt behaviour, and that is because the cognitive process involved is a skill and not a theoretical inference (Wittgenstein 1953/1968).

This may be so, but this is no recipe for developmental psychology, which is, quite rightly, concerned with the neurocognitive processes involved in concept acquisition. For example, it would not do simply to say that after a period of social interaction certain aspects of the child's linguistic environment (prosody, changes in amplitude) become "criterial" for language understanding. Some explanatory hypothesis is required about the nature of the cognitive processes implemented in developing neural architecture. Is the child processing phonology or syntax? How is the relevant information represented and manipulated? It seems uninformative to say that as a result of social interaction children acquire the ability to hear sentences as meaningful, but that is precisely what Wittgenstein said. The best way to comprehend the process is via a metaphor "Light dawns over the whole." But it is simply not true that the psychological explanation of concept acquisition is exhausted by analysis of its phenomenology. And it is doubtful that the philosophical understanding is, either.

Early cognitivists reconstructed these questions as questions about the construction and confirmation by the developing child of theories of the linguistic domain, implemented ultimately in neural architectures. Perhaps this is slightly over-intellectual, but the issue of what information is relevant to cognition of a particular domain and how it is computed is still essential. Furthermore, that is a question about what is going on in the mind of the individual who acquires a language. Putting it this way does not exclude social interaction but it allows us to say why certain aspects of social interaction are more significant than others and why some children rather than others develop the relevant concepts in response to that interaction.

Without such a theory, a linguist would be forced to say that children with, say, Specific Language Impairment (SLI) do not acquire language normally because their linguistic interactions are abnormal. True, but is this because they lack acoustic or phonological information? The ability to process it? Or do they lack some form of grammatical processing? One cannot say that one is concerned only with normal development, because the adequacy of a model of normal development is evidenced by its ability to predict and explain characteristic developmental abnormalities. Similarly for other psychological capacities: much, if not most, of our knowledge of normal function depends on abnormal cases.

The same is true of social understanding. Indeed, the TOM hy- pothesis was originally advanced to explain the difference between normal and autistic children. Perhaps the TOM idea is overly intellectual and perhaps (as the authors argue persuasively) TOM is not a cognitive monolith. In that case autism is unlikely to be a monolith either. But we are then left with the idea that there is a multiplicity of cognitive mechanisms involved in social understanding that reciprocally interact in a developmental cascade in both normal and abnormal cases. Such a view seems entirely consistent with methodological individualism, which is just the attempt to find out what it is about cognitive architecture that enables the normally developing child, first, to become embedded in her social world and, second, to scaffold her development using social interaction. Social exchanges, evidenced in gaze monitoring, social referencing, emotional responses, protodeclarative and imperative pointing, pretence, play, and conversation, all play a role, but the nature of that role is opaque without an understanding of the cognitive mechanisms on which it depends.

The rejection of methodological individualism reflects a debate in cognitive science over the explanation of skills (of which intentional understanding certainly is one). Some argue that "knowhows," the fundamental capacities on which skills depend, should be identified with socially acquired dispositions. Others argue that the acquisition of those dispositions itself depends on some fundamental capacities that are essentially computational and internal to the mind of the individual acquiring the skill. For almost any skill, it turns out that its ultimate explanation is the computational one: Think of recognising faces, catching a ball, learning a language, or playing at dressing up. Furthermore, the phenomenology of skill acquisition is a poor guide to the nature of those computational processes. For this reason, we should be sceptical of any account that is essentially nothing more than an elaboration of phenomenological insights.

\section{The role of executive function in constructing an understanding of mind}

\section{Suzanne Hala}

Department of Psychology, University of Calgary, Calgary, Alberta T2N1N4, Canada. hala@ucalgary.ca http://www.psych.ucalgary.ca/People/Faculty/hala/

Abstract: Adopting a constructivist stance is not irreconcilable with executive function accounts of emerging social understanding. The executive function view allows for a gradual transition in theory of mind, while specifying the underlying cognitive processes that push that development forward. Executive function abilities can be seen as an important interactional component in the epistemic triangle.

Carpendale \& Lewis (C\&L) have done the field a great service in reminding us that the development of social understanding does not take place in a social vacuum. They point out, quite rightly, that the question of importance should not be whether early competence in social understanding exists; rather, the central focus should be turned towards the processes that allow for the emergence of social understanding. The authors propose an alternative constructivist account that promises to bridge the current dichotomy that exists between those who adopt an individualistic developmental approach versus those who subscribe to an enculturation view. The inclusion of Chapman's (1991) "epistemic triangle" helps to further extend Piaget's constructivist theory to the social realm.

Although C\&L's proposal is highly laudable, nevertheless, as was the case with Piaget's original account, important aspects of the developmental process remain underspecified. That is, saying that development is a constructive process tells us little about the exact nature of the cognitive functions that also contribute to development of social understanding. The authors offer coherent objections against three prominent theories that seek to explain 
the emergence of a so-called "theory of mind" in young children: theory-theory, simulation, and modularity accounts. They neglect, however, to address how a constructivist account might be related to a newer, rapidly maturing, alternative theoretical perspective the executive function account.

The term "executive function" broadly refers to those cognitive functions that underlie goal-directed behavior and that are thought to be mediated by the prefrontal cortex (e.g., Welsh et al. 1991). A growing number of researchers maintain that gains made in executive function abilities in the preschool years contribute significantly to theory-of-mind performance (e.g., Carlson \& Moses 2001; Carlson et al. 1998; 2002; Frye et al. 1995b; Hala \& Russell 2001; Hala et al. 2003; Hughes 1998; Russell 1996; Zelazo et al. 1997). As children increase their capacity to control and direct their own actions, they become able to view alternative courses of action - including actions based on beliefs (Russell 1996).

In contrast to the theories criticized by $\mathrm{C} \& \mathrm{~L}$, an executive account can readily incorporate the notion of a more gradual onset of social understanding. Development of both executive control and social understanding begin early and emerge over an extended period of time. Indeed, the precocious performance found in many "modified" false belief and deception tasks (which the authors highlight as increasing personal or social activity) may be explained in terms of reductions in the executive demands of the tasks.

To illustrate: typical false belief tasks draw on at least two executive demands: (1) working memory (of where the object was in the beginning or what the child thought was in a box) combined with (2) inhibitory control (inhibit pointing to the spot where the object is now known to be or reporting what one now knows is really in the box). Recent research has confirmed that those executive tasks that combine both working memory and inhibitory control are most strongly related to theory-of-mind performance (Carlson et al. 2002; Hala et al. 2003). Reducing one or both of these executive demands may result in improved performance. For example, Freeman and Lacohée (1995) found that having children "post" a picture of what they thought was in a box helped them later to recall their own false belief in a contents task. Although personal activity is certainly increased in this version, at the same time so are the executive demands reduced (in this case, working memory). Similarly, reducing the inhibitory demands of deception tasks also results in improved performance (Carlson et al. 1998; Hala \& Russell 2001), whereas simply removing the opponent - and hence reducing the social demands - does not (Hala \& Russell 2001). The reverse pattern is also found. That is, increasing the inhibitory demands of theory-of-mind tasks detracts from performance (Leslie \& Polizzi 1998).

Though I use these examples of modified tasks to illustrate that a more gradual onset of social understanding is consistent with an executive account, I am not claiming that it is simply informationprocessing complexities of specific tasks that stand in the way of young children and their supposed theories of mind. Instead, I, and others, suggest that there is a deeper relation between executive function and developing social understanding. Exactly what this relation is has yet to be specified. Development of executive function may make possible the emergence of a theory of mind (Moses 2001). Alternatively, it may be that a strong relation is consistently found between theory of mind and executive control, not because one is causally implicated in the other in a linear fashion, but because the two are interdependent in their development.

Admittedly, the bulk of the research on the relation between executive function and social understanding is of the individual-differences variety and has not, as yet, wed itself to charting the social interactions the child is surrounded by. In principle, however, the executive and constructivist accounts are not mutually exclusive. Interaction with others challenges children's current executive abilities, and, in Piaget's terms, adaptation in knowledge structures may result, leading to increased knowledge and flexibility in their thinking about their own and others' mental lives. As chil- dren grow in their executive function abilities, they become more adept at interacting with and understanding others.

Introducing executive function ability into the epistemic equation affords a view of the process of development as bidirectional. As has long been maintained by those who adopt a dynamic systems approach (e.g., Bronfenbrenner 1989; Gottlieb 1991; Scarr \& McCartney 1983/1984), the characteristics of the child influence the response of the environment just as the environment influences the child. In this vein, children's executive maturity will, at least in part, influence how their parents respond to them, which in turn will influence and further enhance their developing executive control and social understanding.

\section{ACKNOWLEDGMENT}

The author is supported by a grant from the Natural Sciences and Economic Research Council, Canada.

\section{Understanding self and other}

\section{R. Peter Hobson \\ Developmental Psychology Research Unit, Tavistock Clinic and University College London, London NW3 5BA, United Kingdom. r.Hobson@ucl.ac.uk}

Abstract: Interpersonal understanding is rooted in social engagement. The question is: How? What features of intersubjective coordination are essential for the growth of concepts about the mind, and how does development proceed on this basis? Carpendale \& Lewis (C\&L) offer many telling insights, but their account begs questions about the earliest forms of self-other linkage and differentiation, especially as mediated by processes of identification.

The article by Carpendale \& Lewis (C\&L) is an important corrective to contemporary misconceptions about the development of interpersonal understanding. The authors analyse distortions introduced by individualistic "theory of mind" perspectives that purport to show how children might derive concepts (even concepts of mind) without appropriate forms of interpersonal engagement, and they highlight the equally devastating limitations of simulationist accounts that presuppose understanding of one's own mind as a basis for understanding the minds of others. The arguments they marshal from Wittgenstein and Chapman in particular are, in my view, decisive. As C\&L indicate, a theory in which social exchanges are constitutive of understanding does not lead to cultural relativism. On the contrary, it is only through involvement with others that human beings are in a position to accord objective reality the status it deserves, and only when objective reality is conceived as such do concepts such as "belief" gain a purchase. Moreover, mutual interpersonal relations that entail communication and reference vis-à-vis a shared external world provide a necessary basis for uniquely human ways of (1) acquiring knowledge about that world; (2) understanding what it is to be a person with alternative psychological perspectives on that world; and even (3) thinking symbolically and creatively about people and things and constructing concepts with which to think.

How, then, should we frame our account of early human development if we are to elucidate how all this is possible and how development proceeds from its starting point? Here I wonder whether C\&L are sufficiently radical in their revamping of theory.

Consider how concepts of "self" and "other" are integral to concepts of mind. There is a paradox at the heart of any attempt to reconcile developmental accounts of self-other understanding that focus on the individual's cognitive endowment, on the one hand, and social influences, on the other. The paradox is that an individual has to have bedrock experience of the social as social in order to build upon this to construct progressively elaborated understandings of minds as connected and differentiated centres of consciousness. Without some primitive modes of experiencing self as self in relation to others, and of others as others in relation to self, it is difficult to see how concepts of self and other could be derived. (Note: this does not mean that infants, even infants at the 
end of the first year of life, have concepts or "understandings" of the mental lives of others.)

The direction in which C\&L move on this one, is to stress the activities within which human interaction is embedded, adopting what they call "a relational, action-based perspective" within which communicative interaction also plays an essential role. Yes, but what is the grounding for communication? When the authors state that "Children's social knowledge is based on action" (sect. 3, para. 4), they are in danger of losing the plot. The crux is how infants experience the activities and attitudes of self and other in interpersonal relations, not merely how they act or interact with others. C\&L refer to the embodied nature of human exchanges, but they do little to explain how infants are aware of persons as having a mental as well as physical dimension, and how the nature of this awareness is such as to allow for the partitioning into what belongs to the other and what to the self over successive phases of development. What we need to explain, after all, is how a child comes to understand persons as centres of individual experience, not merely as centres of causality, and how the child's concepts about the different facets of subjective orientation towards the world (intentions, feelings, wishes, beliefs, and so on) develop in the early years of life. Alongside this, we need to account for the forms of reflection and thinking about people - oneself as well as others - that such concepts entail.

I think the solution to the conundrum is that humans are equipped with a propensity for forms of role-taking that both link an individual infant or child or adult with someone else, and at the same time register the distinctiveness of self and other. At first such role-taking is cognitively unelaborated, not yet amounting to understanding: It takes place without pre-existing thought and in a manner that is heavily imbued with emotion. From early in life, children are moved by the attitudes of others: They are drawn to identify with the psychological stance of a person with whom they engage. It is through this mechanism that mutual relations with others vis-à-vis a shared world yield the ability to relate to one's own mental relations, and with this, creates a kind of mental space within which new forms of thinking are possible. A prime example of how individuals interiorize the social, Vygotsky-style.

There is another sense in which this approach is more radical than that of C\&L. These authors give weight to the influence of mother-child as well as peer relations in the ability to acquire and apply concepts of mind. Here the active ingredients of development are conceptualised in terms of cooperative social interaction and exposure to talk about mental states. No doubt these things are important. Beyond this, however, powerful socio-emotional forces are at play.

Especially when you are in the heat of relating to others, it can be an emotionally taxing business to think flexibly and to deploy mental concepts effectively. Critically important for the early development of this capacity are young children's relations with at least one other person who is able to tune into their minds. The developmental influences are not merely intellectual, they are also emotional. Studies in developmental psychopathology reveal that in order to employ mind-related thinking effectively, one needs to do more than construct understanding. To maintain a reflective stance towards one's own and others' minds, one also needs to be in appropriate forms of relation with oneself and others. Emotional relatedness towards persons-as-represented in the mind is a vital force in intrapsychic as well as interpersonal functioning, and such relatedness and representation are powerfully influenced by identification with the attitudes of others.

The sources of mentalistic understanding involve much more than action, even co-action with others.

\section{The sibling relationship as a context for the development of social understanding}

\section{Nina Howe}

Department of Education, Concordia University, Montreal, Quebec H3G 1M8, Canada.nina.howe@education.concordia.ca

Abstract: Carpendale \& Lewis (C\&L) provide a convincing argument for how children construct social understanding through social interaction. Certainly mothers are important in family interaction; however, sibling interaction may also be key in the process of developing social understanding. In particular, the highly affective and reciprocal dynamics of the sibling relationship in both positive and conflictual interaction may be critical.

Jeremy Carpendale and Charlie Lewis (C\&L) have provided an insightful and thoughtful discussion of the development of children's social understanding. To quote these authors, "What benefits can be gained by adopting a constructivist approach to the development of children's social understanding?" (target article, sect. 5). To my mind, there are many. First, as they indicate in their review of the theory of mind (TOM) literature, the focus on the False Belief Task has become very narrow and convoluted in its perspective. Indeed, this literature suffers from the "neurotic task fixation" syndrome that the authors outline. Lab studies can inform us only to a certain degree about children's development and they frequently suffer from overcontrol, a nondevelopmental perspective, and fail to consider the importance of the familial context. Ultimately, such a path may doom the field to a slow and painful death; the history of psychology is littered with many such examples.

However, C\&L provide an escape from this depressing scenario. Humans are social, and therefore to study TOM or, as I prefer, social understanding, within the context of relationships seems ultimately a more satisfying, and theoretically compelling and constructive, approach. By placing children's development within the context of social relationships, the authors provide a framework for their constructivist view of social understanding. As they argue, there is a long tradition of constructivist ideas regarding children's development. Indeed, in the field of early childhood education, this approach has long acquired popularity. The notion that "understanding comes through action" has been a fixture of earlyyears programs for many decades and is one of the basic premises of currently fashionable constructivist programs such as Reggio Emilio's.

Thus, I am in basic agreement with C\&L's premises, but some important points should be emphasized more strongly. Clearly, it is not the quantity but the quality and nature of children's close relationships that are important for social understanding. For more than 20 years, Judy Dunn's work (see Dunn 2002) has suggested that the quality of young children's relationships is related to their social understanding. She has been a leader in reminding us that children's social worlds involve more than just parents and that children spend considerable amounts of time with other youngsters, especially siblings, but also peers. The field of child psychology has suffered from a focus on the mother-child relationship, and, to some degree, C\&L fall into this trap. One of their arguments is that triadic interactions are key to the development of social understanding. Certainly mothers are important (we know very little about fathers in relation to social understanding), but the power of the dyadic sibling relationship should not be overlooked once again. Dunn (2002) emphasizes that particular relationships are important, namely those in which children have a vested interest and which are colored by intense affective dimensions, because these elements may provide the motivation for making one's points understood. The reciprocal nature of the sibling relationship, as opposed to the more complementary or hierarchical parent-child relationship, affords children many opportunities to develop their social understanding in the context of ongoing interaction.

C\&L have identified cooperative parent-child relationships as 
key because they allow for free communication, which should facilitate understanding others' minds. Yet, the nature of complementary relationships by definition places certain constraints on freedom of communication that are not as present in more reciprocal relationships with siblings and peers (Hinde 1979). Furthermore, the authors give little attention to the role of social conflict as another possible context in which children develop social understanding, yet there is a literature providing support for this view (Dunn 2002; Howe et al. 2002; Shantz \& Hartup 1992). In particular, the work that has focused on constructive versus destructive conflicts holds important promise here. In the context of constructive conflict resolution, children must demonstrate the ability to negotiate and resolve disagreements in ways that demonstrate their understanding of the partner's position, often marked by their use of mental state language. Another area that has potential to add to the strength of C\&L's argument is the literature on shared meanings in pretend play. Again, when children have a vested interested in constructing a dynamic play scenario, they must extend and build onto each other's ideas in ways that indicate that they have developed a joint understanding of one another's minds (e.g., Göncü 1993; Lillard 2002). In sum, siblings are frequent partners in this process because, as any parent knows, who is there better to play with and fight with than your sibling?!

Although I have followed the TOM literature for a long time, partly because it has been the fashion leader of social cognition and promised to address some important issues, ultimately it is a narrow literature. I have often wondered what the cognitive shift at age four, as identified through the false belief task, had to do with the real world of children. It took me a long time to make the conceptual connections between TOM and the social-understanding work of Judy Dunn and others. Dunn's work has clearly shown that the development of social understanding is fascinating when we examine children in their real-world context as they (1) make meaningful and practical decisions about advancing their points of view convincingly, and (2) negotiate their way through the complex and rich world of social interactions. Moreover, the development of individual differences in children's social understanding is clearly a fruitful area of future work. For children (and researchers) there is a wealth of fascinating questions and problems to address in figuring out how the social world works and how to understand the depth, breadth, and meaning of one's social relationships. This is a developmental process that probably takes a lifetime, but certainly extends past the age of five. If $C \& L$ have pushed the social cognition field to recognize and address this point, then they will have made a major contribution to the literature.

\section{ACKNOWLEDGMENTS}

The author was supported by a grant from the Social Science and $\mathrm{Hu}-$ manities Research Council of Canada, and thanks William M. Bukowski and Holly Recchia for their helpful comments on the paper.

\section{Emotions and emotion cognition contribute to the construction and understanding of mind}

\section{Carroll E. Izard \\ Department of Psychology, University of Delaware, Newark, DE 10716. izard@udel.edu http://www.udel.edu}

\footnotetext{
Abstract: Carpendale \& Lewis's (C\&L's) interesting and insightful article did not integrate several potentially useful notions from emotion theory and research into their explanatory framework. I propose that emotions are indigenous elements of mind and that children's understanding of them is fundamental to their understanding of the mental life of self and others, understandings critical to the development of social and emotional competence.
}

At several points in their insightful article, Carpendale \& Lewis $(\mathrm{C} \& \mathrm{~L})$ allude to the role of emotions in the construction and understanding of mind. Yet, they typically move on to a discussion of cognitive or social cognitive processes without really integrating any aspect of emotion theory into their conceptual framework. For example, they acknowledge the usefulness of Hobson's (2002) emphasis on the infant's emotional engagement in social interactions. Then, without further reference to the role of emotion, they immediately make the point that the infant's ability to engage in joint attention depends on her understanding of causality. A consideration of the role of emotions in developing such understanding and in constructing and understanding mind might broaden and strengthen their position. Some researchers have shown that people make important decisions, especially in risky situations, largely on the basis of emotion feelings. Such feeling-determined decisions may come after careful cognitive analysis of the consequences (Loewenstein et al. 2001). Others now maintain that brain systems involved in emotions become active when subjects perform tasks related to research on theory of mind (Zimmer 2003).

Thus, both theory and research suggest the possibility of enriching and expanding the authors' attractive concept of the epistemic triangle to create an emotion-cognition triangle. An emotion-cognition account of the construction and understanding of mind would emphasize three principles: (1) emotion feelings are dynamic indigenous components of mind that influence perception and cognition; (2) emotion cognition can shape the world as interesting, friendly, and cooperative, or as stressful, hostile, and competitive; and (3) emotion-related action will emerge as constructive or destructive behavior depending on the effectiveness of arousal regulation, the quality of the thought in emotion-cognition interactions, and the effectiveness with which one modulates the expressions of emotions in words and deeds.

Most of the evidence for the existence of emotion feelings as indigenous components of mind comes from studies of infants' abilities to perceive the facial and vocal expressions of others and to encode their own. Beginning as early as age three or four months, infants discriminate between the facial and vocal expressions of several basic emotions. They respond to them, as well as to different environmental conditions, in ways that suggest they can meaningfully decode external emotion signals and respond sensibly to their own internal signals (Haviland \& Lelwica 1987; Izard et al. 1995; Sullivan \& Lewis 2003; Sullivan et al. 1992; Weinberg \& Tronick 1994). If one accepts these findings as evidence that feelings are natural elements of mind, two things follow. First, as part of the processes involved in the construction of mind, the infant can learn various associations or connections between feelings, expressions, and contingent events (e.g., caregiver actions in response to infant's expressions of feelings). Second, an important part of the sense of self as causal agent can emerge from sensing the contingency of emotion feelings (and expressions) and the changes they induce in the social environment (Tronick et al. 1977; Izard 1978; Malatesta et al. 1989). The processes underlying these developments could surely play a significant part in the construction and understanding of mind. If emotions are indigenous elements of mind, then understanding emotions is tantamount to understanding parts of mind, arguably the parts that drive the workings of the other parts (Izard 2001; Tomkins 1962).

Emotion cognition affects the construction and understanding of mind both in terms of its content and via ongoing information processing. It has two major aspects: (1) understanding or knowledge of emotion feelings, expressions, and functions; and (2) the feeling-influenced information processing mechanisms involved in perception and thought. Emotion knowledge and emotion information processing may play major roles in the development of processes often identified as aspects of theory of mind (Zimmer 2003).

Emotion cognition may have particular consequences for the development of social and emotional competence and the selfconcept. Emotion knowledge (EK) relates positively to social 
competence and inversely to various indexes of internalizing behavior (Denham 1998; Schultz et al. 2001). Indeed, EK in preschool children at risk predicts their social and academic competence in third grade (Izard et al. 2001). By age 11 years, children low in EK see themselves as anxious, depressed, and socially isolated (Fine et al. 2003). Furthermore, given certain environmental conditions, and probably a genetic proneness to negative emotionality, a child can come to see others or their intentions as interesting and friendly, or as hostile or frightening. A strong and persistent association exists between proneness to anger and aggression, on the one hand, and a hostile attribution or anger perception bias, on the other (Crick \& Dodge 1994; Dodge \& Coie 1987; Fine et al., in press; Schultz et al., in press). Thus, emotion perception or attribution biases help determine the content of mind, as well as the direction of emotion-motivated action. These mental biases indicate that things went awry in the development of emotion-cognition relations and the construction of mind.

The association between proneness to anger and anger perception bias illustrates an important emotion-cognition connection. Some research suggests an equally significant connection between emotion, emotion perception bias, and action (e.g., aggression), particularly in peer-rejected children (Hubbard 2001). Their emotion perception bias and related proneness to anger and aggression undoubtedly affect the processes involved in the construction of mind and in understanding the mind of others. Angerand aggression-prone children show a strong tendency to see anger signals and hostile intentions where none exists (Crick \& Dodge 1994; Fine et al., submitted; Shultz et al., in press).

Emotion action is conceived of as overt behavior determined or significantly influenced by emotion feelings. The effectiveness or adaptiveness of emotion action probably depends in large measure on one's success in modulating emotion arousal, particularly intense emotion arousal in stressful social situations (Izard 2002). Strong and persistent expression of negative emotions apparently constitutes mind stuff that may contribute to the development of traits of personality. Negative emotion expression in the more stressful episodes of the Strange Situation (Ainsworth et al. 1978) at age 1.5 years predicted the personality trait of neuroticism at age 3.5 , whereas modulated positive emotion expression predicted conscientiousness and agreeableness (Abe \& Izard 1999). One might expect such traits and their expressions in social interactions and other behavior to figure prominently in the construction and understanding of mind.

\section{The space in between: The development of joint thinking and planning}

\author{
Jennifer M. Jenkins and Keith Oatley \\ Department of Human Development and Applied Psychology, University of \\ Toronto, 252 Bloor St. West, Toronto M5S1V6, Canada. \\ jenny.jenkins@utoronto.ca koatley@oise.utoronto.ca \\ http://fcis.oise.utoronto.ca/ jjenkins/
}

Abstract: We argue that theory-of-mind understanding has developed to facilitate joint thinking and planning, defined as the creation of new mental objects that could not have been created by one mind. Three components of this ability are proposed: the mental architecture indexed by false belief understanding, domain-specific knowledge, and the prioritization of the joint mind over the individual mind.

Introduction. Carpendale \& Lewis (C\&L) are exactly right when they argue for a middle position between individual and enculturation accounts of theory-of-mind understanding. Yet, for the social domain to be seriously considered in this realm where purely cognitive considerations have dominated, one must know which components of theory-of-mind understanding are influenced by individual processes. We suggest that the best way of identifying the components is to argue back from the likely function that theory-of-mind understanding serves in human life.

Our proposal is that theory-of-mind understanding functions to enable the uniquely human activity of joint thinking, which is the creation of new mental objects that could not have been created by one mind, and joint planning, which is the arrangement of plans that could not be accomplished alone. Such joint mental processes are generative. One person's thought or suggested subgoal is conveyed to someone else. The second person takes in the mental object and transforms it. Transformations, slight enough so that new objects can be assimilated by their recipients, provide the creative elements on which culture is based. For Winnicott (1971) they occur in the "space in between" people, a space of possibilities and generativity in which, as a person offers a thought to another person and the thought comes back transformed, something new happens. The building of scientific theory provides an adult example. One scientist offers a theory and another, having assimilated details of that theory, offers a revision. Reading a novel is another example. As the reader engages with the thoughts of the writer, the reader's mind is transformed. In both examples, thoughts are generated that are the product of two minds (Oatley 2003).

False belief should be understood not as a stand-in for theoryof-mind understanding but as one of its components. We suggest that three components enable joint thinking and joint planning, as shown in Figure 1: mental architecture (structure), domain-specific knowledge base (content), and the prioritization of joint goals over individual goals (the motivation). Taking the dimension of individual/enculturation proposed by $\mathrm{C} \& \mathrm{~L}$, evidence suggests that these three aspects have different developmental influences.

Mental architecture. To identify discrepancies between one's own thoughts and those of another, we need simultaneously to represent and compare two potentially conflicting mental objects. This has been assessed by measures of false belief. In contrast to $C \& L$, we suggest that existing data imply that development of false belief is most strongly influenced by individual factors. First, the meta-analysis of Wellman et al. (2001) demonstrated that the age effect in false belief is substantial, unmoderated by other factors, and universal. Hence, biological maturation goes a long way to achieving it. Second, in a sample unrestricted by age (e.g., zero to ten years old) effects of cognitive maturation on false belief would be large and those of social factors, small. Social influences have been shown to speed up acquisition (Cutting \& Dunn 1999; Jenkins \& Astington 1996), but such influences are always demonstrated within a very narrow age range, serving to enhance their relative effect. Third, children without unusual cognitive impairment such as autism, or unusual linguistic experiences such as being deaf with hearing parents, almost all acquire false belief understanding. Except for the unusual cases, by about eight years old there are no individual differences in this skill. Variation in family

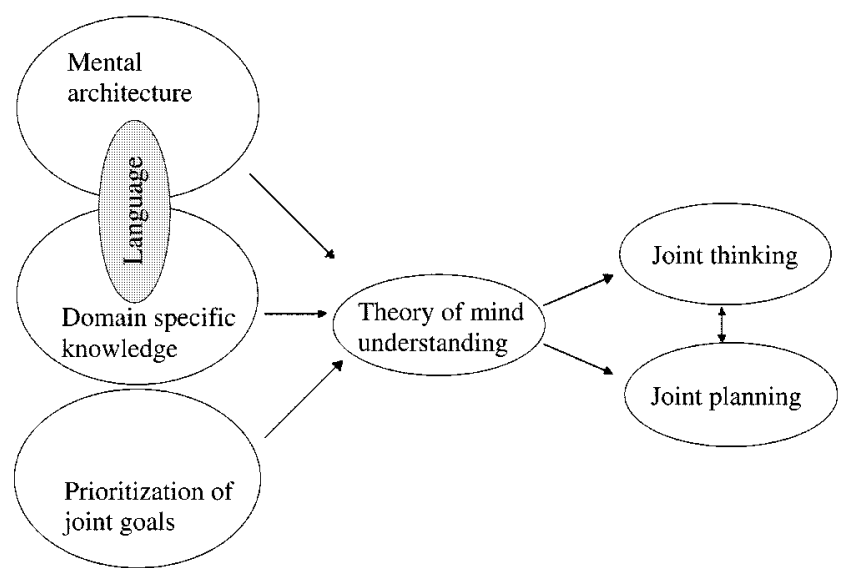

Figure 1 (Jenkins \& Oatley). The components of theory of mind understanding and their role in joint thinking and planning 
and cultural experiences becomes irrelevant: The false belief switch gets turned on anyway.

Language provides some of the architecture necessary for false belief understanding (Astington \& Jenkins 1999; Jenkins \& Astington 1996), and insofar as social factors do speed up the acquisition of false belief understanding, this probably occurs through the effect of social influences on language development. Recent evidence suggests that this may occur through the linguistic structure of complementation (de Villiers \& Pyers 2002). Language also contributes to our next proposed component: the content of domain-specific knowledge.

Domain-specific knowledge. Successful generative thinking and planning between minds requires content knowledge of the domain under discussion. We distinguish two aspects. One is the subject of the discourse: cars, dance, engineering, genetics, and so on. Hutchins (1987) investigated distributed cognition on a navy ship, and reported that successful performance in a seven-person navigation team required that team members had experience of the different roles in the team. This gave them content knowledge to understand ambiguous utterances and to solve problems in a way that was close to the perspective of others. A second aspect is talk about mental processes. If two people misunderstand each other, they may introduce process talk to clarify meaning, intention, and so on. Mental state talk is essential in such clarification: "Do you remember?" "What did you think?" "Are you angry?" The social process suggested by C\&L is clearly central to the development of such content knowledge (Jenkins et al. 2003; Ruffman et al. 2002).

Prioritization of joint goals. Only if one cares enough about the internal experiences of others can the joint goal achieve prominence over one's own goals. Oatley and Larocque (1995) have shown that a whole class of errors in joint planning derives from one person being committed to goals of the self rather than joint goals. Bowlby (1971) described what he called goal-corrected partnerships, in which toddlers represent and act upon the goals of others through having experienced the same thing in their parents' treatment of them. In support of this, Herrera and Dunn (1997) found that those children who had had their own goals recognized by parents and siblings earlier were more likely to play cooperatively with peers two years later. This aspect of theory-ofmind understanding is thus strongly influenced by the social processes outlined by C\&L.

Conclusion. Our delineation of components of theory-of-mind understanding allows for increased conceptual clarity of social antecedents in development. False belief understanding occupies a pivotal role in theory-of-mind development because it enables a type of interaction between minds not otherwise possible (Jenkins \& Astington 2000). False belief and theory of mind are not synonymous. Once false belief has been acquired, other components of theory of mind become more important in explaining why individuals differ in their capacity to enter into the minds of others.

\section{Reconstructing children's understanding of mind: Reflections from the study of atypical development}

\section{Susan R. Leekam \\ Department of Psychology, University of Durham, Durham DH1 3LE, United Kingdom.S.R.Leekam@durham.ac.uk}

\begin{abstract}
Carpendale \& Lewis's (C\&L's) theoretical reconstruction of the "theory of mind" problem offers new hope but still has far to go. The study of atypical development may provide some useful insights for dealing with the work ahead. In particular I discuss three issues - the boundary problem, the question of end states, and the issue of the centrality of triadic interaction.
\end{abstract}

Carpendale \& Lewis (C\&L) proclaim the end of theories of "theories of mind" as we know them. Hoorah! Social interaction wins the vote. Out goes individualism, in comes relationalism. Oldfashioned accounts of understanding mind - theory formation, introspectionism, enculturation, and modularity - are all dead. Long live constructivism! This view is good news for anyone who has ever questioned the primacy of a cognitive account to explain social reasoning. It is also good news for anyone who has ever questioned the lack of developmental emphasis in the study of children's understanding of mind. So C\&L's theoretical reconstruction brings new hope to the theory-of-mind weary.

Now, the next question is how to pull it off. There is much work that still needs to be done. Below I take up three issues - the boundary problem, the question of end states, and the issue of a central role for triadic interaction. For each I suggest ways in which research on atypical populations might give some cautionary insights on how to proceed with the work ahead.

The first issue is the boundary problem - a common theme in the field. C\&L aim to explain how children acquire "social understanding." This broad term refers to all manner of social knowledge, including knowledge of the mind. When it comes to detailing how knowledge is actually constructed within social interaction, wouldn't it help to be specific about the boundaries of that knowledge? Understanding the mind as a representational device may be a very different kind of knowledge than understanding of social rules, or the ability to talk about inner states and the role of social interaction may differ for each of type of knowledge. C\&L seem to agree that different types of social interaction experience may relate differently to particular types of social knowledge when they refer to Peskin and Ardino's (2003) study. But if we want to try to understand better how social interactions actually work in helping to construct different types of knowledge, it may help to study an atypical group with impaired social interaction - children with autism. For example, if children with autism lack basic social relatedness, is it possible that they are ever able to fully grasp the representing function of a symbol or representation, even when such symbols or representations are removed from a social context?

A second problem is how to avoid the notion of "end states." In section 4, C\&L make developmental links between children's early social interaction experiences and later social understanding. This makes social understanding look like an end state or developmental outcome. I do not think this is the intention. On the contrary, what C\&L want to emphasise is "progressivity in development," the idea of a movement away from an initial starting point rather than a directionality towards a predetermined endpoint (Chapman 1988a). But given the way the account is currently formulated, it is difficult to get a sense of the nature of this progressivity. The process of development, as they point out, is a problem. Ideas about development being embedded in activity, regularity, social practice, or conversations and involving transactions between self and others have a long way to go. And we probably need to start at the beginning and look forward rather than trying to explain social understanding backwards.

The study of atypical development may offer some insights here. The way that development is modelled in neuroconstructivist accounts of atypical development (Thomas \& KarmiloffSmith 2002) is to examine how precursor states may be related to particular outcomes given different sets of constraints or circumstances. Although the idea of predetermined end-states might not appeal, connectionist dynamic systems and transactional accounts all argue that development is emergent, that influences are bidirectional rather than unidirectional, and that development involves a series of changes in a self-organising system. Capacities may disappear and then reappear later, may peak and then decline with time, and may start as general and become more specific. Organismic and systems theories also propose changes that include integration of earlier accomplishments as experiences and abilities are integrated into the subsequent reorganisation of the system.

A fuller account is still needed of the way in which developmental change proceeds over time from the starting state of social interaction. However, the idea that development involves an integration or reorganisation of earlier accomplishments is high- 
lighted in C\&L's discussion of the epistemic triangle. Triadic interaction is given a central role in this account as it incorporates and integrates more basic dyadic skills. The centrality of triadic interaction is consistent with a view of development in which one form of knowledge is considered more complete or more adequate than other forms of knowledge. But how central is the role of triadic interaction? Does it supplant other forms of activity and knowledge? Does it remain central throughout development? Our research in autism (Leekam \& Ramsden 2003) indicates that while triadic interaction difficulties are critically important, dyadic interaction difficulties alone are a very powerful indicator of language and cognitive skills. Atypical groups, therefore, may give further insights into the continuity and discontinuity of link between dyadic and triadic skills. For example, children with autism have high levels of engagement with objects, whereas, in contrast, children with Williams syndrome have high levels of dyadic engagement with people and poor non-social knowledge. Both groups have difficulties with triadic interaction. What kind of understanding can be constructed from these different starting points and how is the ongoing development of this understanding constrained, facilitated, or transformed by experiences across time? C\&L's account may not be able to answer these questions yet but it provides a good starting point for constructing a new understanding of children's understanding of mind.

\section{Rich interactions and poor theories}

\section{Orlando M. Lourenço \\ Department of Psychology, University of Lisbon, 1649-013 Lisbon, Portugal. Orlando@fc.ul.pt}

Abstract: Carpendale \& Lewis's (C\&L's) critique of traditional accounts of "theory of mind" is well taken, but the alternative theory they propose is premature at its best, unconvincing at its worst. The proposed theory is ad hoc and confirmatory in its findings; vague and generic in its claims; and unjustified and unnecessary in its (putative) novelty.

Critic John Horgan (1999) recently commented discouragingly about progress in psychology: "Theories of human nature never really die; they just go in and out of fashion” (pp. 6-7).

I am extremely sympathetic to Carpendale \& Lewis' (C\&L's) paper because it presents a scholarly review of the literature on children's understanding of the mind. However, the authors' alternative theory seems so ad hoc and vague that it conforms to Horgan's critique.

Ad hoc theory. When a new theory is proposed, one expects that its authors will contrast the theory's ability to deal with empirical findings against the ability of alternative theories. When this is not the case, the findings invoked to support the new theory are not critical, but confirmatory. In the present case, the findings invoked by C\&L are consistent not only with the authors' new theory, but also with the approaches they criticize, and even with theories the authors do not mention. For example, if the "sibling effect" (with all its qualifications) found in children's social understanding may be interpreted in terms of C\&L's account, it may be interpreted also in terms of (1) the theory-theory view, which states that theories are revised because, among other things, "children ... depend on a social world ... for much of the information they will use in theory construction" (Gopnik \& Meltzoff 1998, p. 19); (2) the simulation theory, which states that interactions among children help them understand their own and others' mental states (Harris 1991); (3) the enculturation perspective, which claims that "children internalize the folk psychology of their particular culture" (Astington \& Olson 1995, p. 184); (4) the experiential approach, which "takes into account both the conditions of experience in the specific social and cultural world, and also the phenomenology of experiencing on the part of the child" (Nelson et al. 2003, p. 25); and (5) even modular theories for which "there is nothing in the notion of modularity that prevents even matured modules from learning and developing” (Scholl \& Leslie 2001, p. 698). More generally, when findings are interpreted a posteriori they can be made to accord with almost any theory. Hence, C\&L's theory reminds us more of an approach to which data have to conform than one that conforms to the data.

Vague theory. C\&L's main proposition is that the development of children's social understanding occurs within a triadic interaction involving the child's experience of the world as well as communicative interaction with others about their experience and beliefs (i.e., Chapman's [1991] epistemic triangle). Although true and of some heuristic value, this proposition is inherently vague. Without further qualitative or, better still, quantitative specifications, the proposition can be used, as is often the case throughout the paper, to make almost any claim, to generate almost any prediction, to explain almost any finding, to describe almost any psychological process, and to be applied to almost any developmental phenomenon. For example, it is hardly new - perhaps it is plain common sense - that the psychological development of children involves an activity matrix made up of biological, social-cultural, and psychological dimensions. In the same vein, to expect that children do better on false belief tasks when they are actively involved; to say that conversation about the mental world may be essential for the development of social understanding; and to declare that the extent and nature of social interactions experienced by children facilitates their development of social knowledge, hardly raises above common sense. What seems to be the greatness of C\&L's theory - its apparent consistency with countless findings, authors, and theories - may be the hallmark of its fragility, for only at the cost of remaining at a generic level can the theory accord with almost anything psychological.

Consider the following example. C\&L maintain that although Thelen and Smith's dynamic systems approach is in many ways consistent with their account, the ideas of interactionism and transactionalism make their account distinctly different. For, in contrast with traditional children's "theories of mind," C\&L's account integrates the social and individual dimensions of development. However, because the two key concepts of interactionism and transactionalism were not specified, the authors' claim would certainly be classified as vacuous by Thelen and Smith: "Interactionism and transactionalism are everyone's comfortable buzzwords, and the proffered 'solution' to the nature-nurture dichotomy [and many others]" (1994, p. xv).

Unnecessary theory. C\&L's account boils down to the idea that children's social understanding occurs within social interaction, is gradual, and involves an active subject. But when these claims remain at a simply verbal level they will not be powerful enough to sustain an alternative theory of individuals' social understanding, regardless of how flawed previous accounts may be. Without additional specifications and elaborations, Piaget's appeal to constructivism and gradualism and Vygotsky's focus on social interaction do not need to be repeated. In addition, they do not justify yet another theory on children's social understanding. Because all subjects behave and while behaving must perforce be active, we should be careful when proposing theories whose essence relies on the contrast between an active and a passive individual. What counts as a subject's passive or active role in his or her psychological functioning may be more a semantic problem - all too common in disputes among weak psychological theories - than a substantial problem (see Lourenço 2001).

Regarding the necessity of C\&L's account, I believe it does not go any further in terms of claims, processes, or predictions than previous developmental theories, such as those of Piaget, Vygotsky, or Selman. For example, because Selman's (1980) theory identifies five levels in individuals' interpersonal understanding, and also appeals to the idea of an active and interactive child, it goes well beyond C\&L's seemingly distinctive idea of the emergence of an interpretive theory of mind, which according to these authors would be the second (and last) level in individuals' understanding of the mind.

It might be argued that even if C\&L's approach were reducible 
to previous ones, the account would still be necessary because it allows us to explore five areas of urgently needed research, one of which would be a detailed analysis of infants' interaction. Ironically, C\&L are the first to recognize that some contemporary research of this sort already exists.

In summary, C\&L's critique of traditional accounts of "theory of mind" is correct. However, in its present state their new theory is clearly premature - unless, that is, one favors the proliferation of weak psychological theories and the perpetuation of what Meehl (1978) has called the slow progress of soft psychology.

\section{ACKNOWLEDGMENTS}

I thank Armando Machado and Olga Pombo for their comments on an earlier version of this commentary.

\section{Constructing agents: Rethinking the how and what in developmental theories of social understanding}

\section{Victoria McGeer \\ Philosophy Program, RSSS, Australian National University, Canberra, ACT 0200, Australia.mcgeer@coombs.anu.edu.au}

http://www.sfu.ca/neurophilosophy/members/mcgeer/index.htm

\begin{abstract}
Although I am broadly in sympathy with Carpendale \& Lewis's (C\&L's) version of social constructivism, I raise two issues they might address. One bears on the question of how social understanding develops: Is their resistance to individualism inappropriately combined with a resistance to internalism? A second question concerns a more radical implication of their view for what social understanding is.
\end{abstract}

Three cheers for Carpendale \& Lewis's (C\&L's) "constructivist" approach to the development of social understanding, with its insistence on the gradualistic, action-oriented, and socially embedded nature of this process. In C\&L's view, developing children become, not theoretically competent in a body of folk-psychological (FP) knowledge, but skilled social beings, able to demonstrate in myriad ways their capacity-in-action to negotiate the complex, normatively structured social world in which they and their fellows are embedded. I am broadly in sympathy with this account but raise two issues C\&L might address. One bears on the question of how social understanding develops, the other on what social understanding is.

Let me first address the question of how social understanding develops. By taking the skills approach, $C \& L$ are able to chart a constructivist middle way between the extremes of "individualism," which credits the child with having or doing too much in development, and collectivism, which credits the child with having or doing too little. Yet despite C\&L's focus on how development occurs - in particular, given their resistance to a passive and wholly mysterious view of social enculturation - their own account of this process, especially in early infancy, is mechanistically vague. How do self-other differentiation and coordination gradually emerge within the context of parentally supported dyadic and triadic interactions? It has something to do with the infant's developing and combining sensorimotor schemes, we are told, but the "riddle" of this emergence has yet to be solved.

Where do we look for the answer? C\&L suggest it will be found in more detailed longitudinal analysis of behaviours that emerge in the context of such interactions. Although no doubt helpful, this leaves the mechanistic side of this puzzle quite untouched. I worry that C\&L's justifiable anti-individualism may seem to blend into less-justified anti-internalism, a resistance to investigating what cognitive/perceptual mechanisms within the infant could support the gradual emergence of progressively more skilled social behaviours.

Such mechanisms need not be so richly specified as to amount to a prewired capacity for social understanding, nor need they be such as to presuppose sophisticated (theory-like) processing of others' intentional behaviour as such. They may be quite low-level mechanisms: for example, the mechanisms underlying early imitation, allowing infants to cross-modally map their own proprioceptively experienced bodily movements onto like movements that they see performed by others (Meltzoff 1990; Meltzoff \& Gopnik 1993; Meltzoff \& Moore 1992). The progressive development and combination of sensorimotor schemes would then be explained, in the first instance, by the infant's primordial perceptual urge to map what others do onto what they do themselves. Of course, this perceptual urge may soon be reinforced by the other benefits that infants gain through such interactions - for example, epistemic benefits that derive from adults' intentional scaffolding, but also, perhaps at first more importantly, the sensory/affective regulative benefits suggested by $\mathrm{C} \& \mathrm{~L}$ in connection with a lean interpretation of early joint attention behaviour (Baldwin \& Moses 1996; Gergely \& Watson 1996; McGeer 2001).

However, my point here is not to push any particular view, but to query C\&L about their general attitude towards internalism (versus individualism). In connection with this, it is interesting to note a parallel "neuroconstructivist" movement focused on internal structures and mechanisms that attempts to chart a middle way between the extremes of nativism (too much prespecified structure) and empiricism (too little structure) to explain the development of higher-order capacities through the child's interaction with a progressively structured, even necessarily social environment (Elman et al. 1996; Karmiloff-Smith 1998; Quartz \& Sejnowsky 1997). Given the similarity of constructivist ambitions, there may be fruitful connections to exploit between these two programs.

Now to the question of what social understanding is. I begin by noting that once the resistance to individualism is clearly separated from a needless resistance to internalism, C\&L may well avail themselves of a better way to characterize the infant's starting state than one of relative nondifferentiation between self, other, and world. However true this characterization may be, it suggests a lack of structure and/or activity on the infant's part that seems to fit better with the enculturation model C\&L rightly reject. The infant is not a passive receptacle but active in constructing skills in and through social relationships that eventually constitute the skills of a genuine folk-psychological agent, that is, of a person who manifests his or her social understanding in myriad skillful ways of interpreting others and acting in the normative terms of folk-psychology.

The developmental challenge is therefore not so much one of "differentiation" as it is of transforming/having transformed early meaningless activities into the progressively more meaningful activities of a well-behaved folk-psychological agent. This characterization may constitute a subtle shift of emphasis, but its point is to bring out an important implication of C\&L's approach not fully highlighted in their target article - namely, that "social understanding" is not just a body of knowledge, even in the sense of know-how, allowing individuals to understand one another as intentional agents. It is, more significantly, a regulative practice that is, a complex set of skills by means of which individuals regulate themselves in accord with the norms of folk-psychology, thereby themselves becoming well-formed agents from the folkpsychological point of view (McGeer \& Pettit 2002). The development of "social understanding" is therefore, in actuality, the development of social agency: a progressive enablement of individuals' agential skills by means of which they are also progressively empowered to act and react in self-standing ways within a community of others. All this is quite compatible with C\&L's program: It merely raises the question of whether their substitution of the phrase "social understanding" for "theory of mind" goes far enough in capturing their sense of what children are constructing within social interaction.

\section{ACKNOWLEDGMENT}

This commentary was supported by the McDonnell Foundation through a grant received from the McDonnell Project in Philosophy and the Neurosciences. 


\section{Infants' minds, mothers' minds, and other minds: How individual differences in caregivers affect the co-construction of mind}

\section{Elizabeth Meins}

Psychology Department, University of Durham, Science Laboratories, South Road, Durham DH1 3LE, United Kingdom. elizabeth.meins@durham.ac.uk

\begin{abstract}
Carpendale \& Lewis’s (C\&L's) constructivist account needs greater emphasis on how individual differences in caregivers' impact on the efficacy of epistemic triangle interaction in fostering children's understanding of mind. Caregivers' attunement to their infants' mental states and their willingness to enable infants to participate in exchanges about the mind are posited as important determinants of effective epistemic triangle interaction.
\end{abstract}

Carpendale \& Lewis (C\&L) argue that children are active participants in acquiring mentalising abilities, constructing an understanding of mind during social interaction within the epistemic triangle. Their account represents an advance on individualistic and enculturation approaches to theory of mind (ToM) development, but would benefit from a greater consideration of how individual differences in caregivers influence the efficacy of interaction in the epistemic triangle in fostering an understanding of mind. In order for children to benefit most from social interaction in constructing a ToM, the interlocutor should be sensitive to and cognisant of the child's current state of mind. For example, in one of our longitudinal studies on the social determinants of ToM performance, we found that mothers' use of mental state language that commented appropriately on their 6-month-olds' putative mental states was an independent predictor of children's ToM performance at age 4 (Meins et al. 2002). In contrast, indices of the general quality of the mother-infant relationship (maternal sensitivity and attachment security) did not predict children's subsequent ToM.

What is perhaps most interesting about this study's findings is that ToM performance was related to only certain kinds of early mental state language, and not to exposure to mental state talk in general. Mothers' use of mental state language that appeared inappropriate to the infant's current mental state, indicating that they were misreading their infants' minds, was unrelated to later ToM understanding. Hence, although C\&L claim that their "approach to the development of children's social understanding focuses on the relations between people" (sect. 2.1, para. 8, emphasis in original), they need to move beyond the assumption that the same form of relationship (e.g., mother-infant, child-sibling) will result in the same form of interaction. At present, $C \& L$ provide a detailed description of prerequisites required by the child to engage in constructive interaction in the epistemic triangle (e.g., joint attention skills, a certain level of linguistic competence), but individual differences in caregivers are not considered. Their account therefore places too much burden on the child's attributes in explaining individual differences in ToM understanding. Indeed, C\&L need to consider the possibility that the child's attributes may initially be rather unimportant beyond giving the caregiver an indication of basic competence.

There is also the issue of timescale. In setting up the epistemic triangle as the context in which children construct an understanding of mind, the authors focus exclusively on infancy. Yet, they seek to use their account to explain social influences on ToM that come into play much later in development (sect. 4.2). For example, the facilitative effects of sibling (Dunn et al. 1991b) and peer (Brown et al. 1996) interactions have been found only in the preschool period, and the sorts of parenting style found to relate to ToM performance (Ruffman et al. 1999) would appear to be applicable only to children beyond infancy. Preschool children's social interactions involve complex abilities, such as pretense, with perhaps several playmates, whereas the classic epistemic triangle interaction involves a much more pared-down form of triadic exchange. It therefore seems that in focusing on preschool influ- ences, C\&L are trying to explain the "wrong" evidence. If epistemic triangle interaction is the means by which children construct a ToM, the authors need to concentrate more clearly on social-environmental factors that act during an earlier period of development.

Of course, this is a difficult task, because very little long-term longitudinal research on the social determinants of ToM exists. C\&L mention one early social factor that has been linked to ToM (attachment security), but they need a much more thorough critical appraisal of how such differences in attachment are related to the child's active construction of mind. For example, no author has proposed a direct link between attachment security and children's ToM. Rather, certain precursors of attachment security, such as maternal mind-mindedness (Lundy 2003; Meins et al. 2001) or mothers' internal working models of attachment relationships (Fonagy et al. 1991), are likely to be at the root of any observed security-related differences in ToM. The epistemic triangle is an ideal context for highlighting how caregiver attributes - their mind-mindedness, their willingness to interpret their infants' behaviours as having intention, their representations of their own childhood experiences - are brought to bear on what they say to their infants and how they manage early dyadic and triadic interactions. Such a focus would also enable C\&L to discuss in greater detail how atypical development (e.g., deafness, autism) may affect the caregiver's ability to interact within the epistemic triangle, and thus, children's ToM development.

In order for the constructivist account to explain how the social environment influences ToM, it needs to address how the attributes of the caregiver work in concert with those of the infant to provide early interactions that will foster the child's understanding of mind. Such interactions need to do more than merely ensure that the child is exposed to mental state language. It is likely that our finding that mothers' mind-related language at 6 months predicts later ToM performance is due in part to the fact that such language is one facet of a broader picture of general attunement between mother and infant (e.g., Lundy 2003). An insufficient attention to the child- and caregiver-centred determinants of this attunement means that C\&L's account is in danger of suffering from the very failing that they complain about in traditional accounts of ToM development, namely, the lack of an "integrated system" within which social-environmental influences on ToM can be understood. A more careful emphasis on caregivers' attributes (as well as those of the child), and their willingness to allow children to participate in exchanges about the mind, would provide the authors' account with precisely such integration with the wider context of social-cognitive development.

\section{Structure, genesis, and criteria}

\section{Carol A. Millera and Ulrich Müller ${ }^{b}$}

a Department of Communication Sciences and Disorders, Pennsylvania State University, University Park, PA 16802; ${ }^{\mathrm{b}}$ Department of Psychology,

Pennsylvania State University, University Park, PA 16802.

cam47@psu.eduｕmueller@psu.edu

Abstract: We agree that social interaction is crucial for understanding the development of theory of mind, but suggest that further elaboration of certain issues is needed. Detailed description of the knowledge structure of a developing theory of mind is necessary, and the notion of criteria for the use of mental state terms requires consideration of the sentence structures in which such terms appear.

Carpendale \& Lewis $(\mathrm{C} \& \mathrm{~L})$ make a timely contribution to current debates regarding the development of theory of mind. C\&L's emphasis on the gradual development of theory of mind in the context of interactions between child, other, and object provides a much-needed balance to the extremes of current accounts of theory of mind development, which focus narrowly on processes 
within the individual, or the cultural construction of psychological states, and ignore the interface between individual and culture. However, we contend that C\&L's approach needs to be extended and made more precise in order to fulfill its explanatory potential. Two issues in particular are in need of elaboration: The first issue pertains to the relation between structure and genesis, and the second to the concept of criteria.

C\&L provide a genetic account of the development of social understanding in general, and theory of mind in particular. Their proposal, however, lacks any detailed description of the knowledge structure that characterizes different levels of social understanding. They briefly allude to three features that are required for the acquisition of a theory of mind: (1) the assumption of a stable, external world; (2) the importance of the realization that there is access to information through seeing; and (3) attentional capacity. None of these features, however, clarifies how understanding of, say, false beliefs differs from understanding of, say, the concept of seeing, or why the former is acquired at a later age than the latter. To the extent that C\&L focus on the developmental mechanisms that lead to the acquisition of a theory of mind, their proposal is, in fact, compatible with a number of proposals that focus more on the knowledge structure involved in theory of mind. Other proposals have tied this knowledge structure to metarepresentation (Perner 1991), confronting of perspectives (Perner et al. 2002), the embedding of if-if-then rules (Zelazo \& Frye 1997), working memory (Gordon \& Olson 1998), inhibition (Carlson \& Moses 2001), and mastery of sentence complements (deVilliers \& deVilliers 2000).

Without a more detailed description of what is involved in a theory of mind, the specific forms of social interaction that may promote or impede the development of a theory of mind (Turnbull \& Carpendale 1999a) remain underspecified. It is possible that different aspects of social interaction contribute to different aspects of the knowledge structure characteristic of theory of mind. For example, whereas the coordination of conversational turns may promote the increase of working memory, contrastive utterances may facilitate perspective-taking (Sabbagh \& Callanan 1998). The forging of closer links between social interactions and the development of a theory of mind requires detailed description of what is involved in the acquisition of a theory of mind. In fact, the close linkage of structure and genesis is one of the main theses of Piaget's theory (1967, p. 147): "Genesis emanates from a structure and culminates in another structure" (emphasis in original).

C\&L suggest that mental state terms are learned by observing "the patterns of activity that are criterial for the use of such mental state terms - that is, the pattern of interaction for which we use these words" (sect. 3.2, last para.). Following Wittgenstein, they reject the notion that mental state terms point to inner objects. However, the same arguments against the view of words as correlated with things also apply to the view of words as correlated with events, that is, "patterns of activity." Lila Gleitman and colleagues have cogently presented the case that words are not simply mapped onto events in the world (Gillette et al. 1999; Gleitman 1990; Landau \& Gleitman 1985). If a learner were to try to match a single word to its contingencies in the world, the possible hypotheses of the word's meaning are, if not infinite, certainly intractable, especially for verbs. Mental state terms would be particularly difficult to learn through word-to-world matching, as psychological states are not directly observable.

In other work, Carpendale argues that in order to understand the role of talk in the development of theory of mind, one must set aside the traditional "code model" of language (Turnbull \& Carpendale 1999a). We would argue here that although "talk-asinteraction" is crucial to the development of theory of mind, the language code itself is also important and must be integrated into an overall account of social interaction and theory of mind. The structure of language is as important for learners as the way language is used.

Consider the verb to think. The number of "patterns of activ- ity" that could reasonably occur in social interactions at about the time when an adult utters the word think is huge. What common elements could be extracted from those situations and taken to constitute the criteria for the use of think? Worse yet, thinking is going on all the time, yet talking about thinking is not. L. Gleitman and colleagues (e.g., Gillette et al. 1999) have suggested that the child can solve these difficulties by using, among other sources of information, the structure of the sentences in which mental state terms appear as a cue to their meanings. For instance, mental state terms and communication terms take propositions as arguments. By noting the range of sentence structures in which a verb appears, the child can gain crucial information about its meaning, for example, that mental states relate to propositions.

As C\&L point out, "language, or communicative interaction, is the means through which children learn about other people's experience and so develop a more complete set of criteria" (sect. 3.2, para. 9). That set will not be complete unless it includes the sentence structures in which mental terms appear. We are very much in sympathy with C\&L's approach to the study of theory of mind. We suggest that a first step in moving the approach forward is a more elaborated understanding of the relation between structure and genesis, and consideration of both the linguistic and nonlinguistic contexts in which mental state terms are used.

\section{Being able to understand minds does not result from a conceptual shift}

\section{Peter Mitchell}

School of Psychology, University of Nottingham, University Park, Nottingham NG7 2RD, United Kingdom. peter.Mitchell@nottingham.ac.uk http://www.psychology.nottingham.ac.uk/staff/plm/home.html

Abstract: If anything, Carpendale \& Lewis's (C\&L's) target article could have gone even further in challenging the view that a radical conceptual shift equips children with a theory of mind. Also, the authors should have elaborated on why their social constructivist account is more plausible than nativism. Their argument against simulation theory is perhaps the leastdeveloped part of their thesis, and does little service to their cause.

Carpendale \& Lewis's (C\&L's) thought-provoking target article offers a compelling account of the development of an understanding of the mind, and will be welcomed as a refreshing and attractive alternative to the rather ubiquitous "theory-theory" and modular accounts. On finding myself broadly in agreement with most of the views expressed in the article, I shall confine comments to matters of emphasis and the few areas of disagreement.

First, though, the most significant aspect of the article deserves to be highlighted. The authors provide a convincing argument on the gradual character of development in this important sphere of human functioning. On face value alone, it seems much more plausible than the popular claim from theory-theory that children undergo a singular radical conceptual shift. As Chandler and Hala (1994) persuasively argued, there are much more important and interesting changes that occur both before children begin passing a traditional test of false belief, and indeed afterwards. This brings into focus a detrimental consequence of theory-theory's dominating position as the mainstream account: Attention has concentrated so heavily on what happens at four years of age that it has effectively blinded many researchers to developments in understanding the mind that occur both before and after this time. If C\&L stimulate attention to developments taking place at these other times, it will have done a great service. Indeed, they could have been even more ambitious and ventured further into the territory of development beyond the age of four years.

Another aspect of the article that needs to be highlighted is the much-welcomed critique of Wellman et al.'s (2001) claim to have discovered the truth about false belief. C\&L correctly say that these authors set up a straw person as an alternative to their (Well- 
man et al.'s) favoured conceptual change account. Here, C\&L show restraint, for they could have pointed out that Wellman et al. were highly selective in stating developmental trends that would be predicted by rival accounts. Of course, it came as no surprise to find that children begin systematically giving incorrect judgments in a test of false belief and then gradually change over a period of months such that the probability of giving a correct judgment increases monotonically. In this context, C\&L should have pointed out two obvious things:

1. Anyone who predicts sharp conceptual change should not be predicting a monotonic trend (as Wellman et al. curiously did), but an S-shaped function. That is, children should be predicted by this brand of theory-theory to begin consistently giving an incorrect judgment, and then they should suddenly shift to giving correct judgments, and, on reaching that plateau, continue thereafter with few or no errors.

2. Those theory-theorists who believe in a radical conceptual shift seem not to have a satisfactory account of children's systematically incorrect judgments in a test of false belief (but see Perner 1988; Wimmer \& Hartl 1991). This is something that C\&L also neglect, though in fairness the phenomenon does not threaten their account.

To get a sense of how problematical it is for an advocate of conceptual change, one need only look at the way Wellman and colleagues struggle to provide a description of children who perform below ceiling level. The trouble is that a theory-theorist is compelled to say that a child who performs below-ceiling lacks competence. But then Wellman and colleagues find they need to acknowledge two kinds of incompetence. One is the incompetence that prevents some children from responding systematically (i.e., they are correct about $50 \%$ of the time), and the other is the incompetence that afflicts even younger children in a way that leads them to be systematically incorrect. Now, if children are responding systematically (albeit incorrectly), then, according to the principles of theory-theory, it surely implies that they are acting upon a strategy. Acting upon a strategy seems to be more advanced with respect to theory-theory than not acting upon a strategy; therefore, a theory-theorist should predict that being correct $50 \%$ of the time, where there is no evidence of strategy, should developmentally precede being correct systematically less than $50 \%$ of the time, where there is evidence of (incorrect) strategy. This kind of U-shaped development is what Wellman et al. should have predicted. But if they had done so, then they would have to face a disappointing refutation when looking at the results of their metaanalysis.

C\&L focus on aspects of early social interactions and how these are vital for children to construct an understanding of the mind. This well-formulated thesis deservedly takes centre stage in their account, but some of the evidence pointing to the level of proficiency in children's early social functioning could be interpreted as a sign that they are even more able than C\&L recognize. The authors interpret the proficiency as a precursor of later developments; they see these abilities as being necessary ingredients for quality social interactions, and, in turn, the ensuing interactions will supply the grist from which children construct a better-developed understanding of the mind. Another possibility, though, is that these are signs of an early understanding of the mind rather than precursors. In other words, although C\&L do an excellent job of challenging theory-theories that are premised on conceptual change, perhaps their arguments against accounts of early competence are somewhat less elaborated.

Similarly, their argument against simulation theory is a lesser feature of their article, being premised on the straw person of introspectionism. Actually, simulation theory can posit that in placing yourself imaginatively into another person's (e.g., John's) perspective, you orient outward from that vantage point to see how the world appears (e.g., Currie \& Ravenscroft 2002; Heal 1996). On finding that the world looks like $\mathrm{X}$, you then use an ascent routine: I believe $\mathrm{X}$. Then you step out of the imaginative mode and can impute John with belief X. Clearly, this process does not comfortably fit the description, "introspection."

In summary, $C \& L$ do an excellent job of championing an account of gradual development. Their account is refreshing and compelling in its focus on the value of early triadic relationships. I think that their account would have been even more powerful if some of the emphases had been slightly different, but notwithstanding, they have done a great service in generating a cogent alternative perspective to the mainstream.

\section{Challenging theory-theory accounts of social understanding: Where is the social constructivist advantage?}

\author{
Derek E. Montgomery \\ Department of Psychology, Bradley University, Peoria, IL 61625. \\ montg@bradley.edu
}

Abstract: Carpendale \& Lewis (C\&L) contend that correlations between sociolinguistic factors and theory-of-mind performance indicate that social knowledge develops from social interactive processes. However, theory-theory proponents also regard these correlations as compatible with their view of how mental concepts develop. A more fruitful distinction lies in the differences of both accounts in explaining how mental concepts acquire meaning.

Carpendale \& Lewis (C\&L) provide a welcome alternative to individualist and enculturation accounts of the development of social understanding. They argue that children's mentalistic concepts, at the center of social understanding, are neither passively acquired conventions nor theoretical postulates individually constructed through inference and introspection. Instead, these constructs originate in the crucible of everyday social activities and exchanges.

Two issues in this area stand out as fundamental: (1) to what extent mental states are understood by young children as theoretical entities with causal properties, and (2) the mechanisms by which children develop an understanding of the mind. Regarding the second issue, C\&L conclude that mentalistic knowledge develops largely through interpersonal rather than intrapersonal mechanisms. Consequently, they see the correlations between sociolinguistic factors and theory-of-mind task performance as clearly playing to the advantage of social constructivist theories (see, especially, sect. 4.2). This advantage, however, may be based to an extent on an incomplete picture of the theory-view. At one point C\&L write, "children's social knowledge is based on action; it is not theoretical in the sense of a set of laws formed on the basis of observation to explain the doings of other people" (sect. 3 , para. 4) (emphasis mine). This is slightly misleading since the theory-view does not explain theories as forming simply from observation. Instead, the child's social activity presents a data set which influences the development of mental concepts. Bartsch and Wellman (1995), for instance, regard conversations about causality and about conflicting desires as being directly related to the development of a theory of mind because these conversations help children to grasp that mental states are subjective causal entities. Theory-theory proponents, like social constructivists, regard sociolinguistic influences on individual differences in theory-of-mind understanding as being complementary to their own view. Wellman and Gelman (1998, p. 542) claim quite plainly that children "who are exposed to very different amounts of mental state data should acquire mental state understandings on different timetables."

The first of the two fundamental issues mentioned earlier - how children regard the ontological status of mental state concepts is the fault line where social constructivist and theory-theory accounts most clearly divide. Theory-theory accounts assume that 
Commentary/Carpendale \& Lewis: Constructing an understanding of mind

children regard mental states as private and internal sensations that are causally related to, and distinct from, outward behavior and other mental states. The meaning of children's language about the mind is derived from its referential association to these corresponding mental concepts. But this picture is essentially wrong from a social constructivist view, for a number of reasons (see Montgomery 2002), some of which are discussed by C\&L (sects. 2.2 and 3.2). One problem is that the theory view lacks a plausible account of how a meaningful language could be developed by a process of labeling private and internal referents. For example, obstacles to word learning arising from the indeterminate nature of referential labeling are only exacerbated when the referent is both internal and private, and it is unclear from the theory-theory position how children would overcome such obstacles (Montgomery 2002).

Figuring out word-referent relations for various mental states is not a fundamental component of social understanding. Children learn about the mind through communicative interactions, and linguistic meaning follows from how mental verbs are used in discourse rather than from the nature of a referent they are labeling. Indeed, Wittgenstein (1958, para. 293) suggests that the nature of a referent can be irrelevant to linguistic use:

Suppose everyone had a box with something in it: we call it a "beetle." No one can look into anyone else's box, and everyone says he knows what a beetle is only by looking at his beetle-Here it would be quite possible for everyone to have something quite different in his box. One might even imagine such a thing constantly changing-But suppose the word "beetle" had a use in these people's language?-If so it would not be used as the name of a thing. The thing in the box has no place in the language-game at all; not even as a something: for the box might even be empty.

What communicative end is achieved by pointing, in a sense, to an internal sensation? How often does a child simply say, "I wanna," where the communicative end is simply to reveal that the speaker is presently experiencing the sensation of desire? One can imagine such an utterance as making sense only in the context of obtainment. The necessity of explicitly or implicitly attaching the verb to a complement, "I want that x," underscores that the central communicative end of using "want" is to express obtainment.

Understanding the pragmatic ends toward which mental verbs are aimed is a critical plank in the research agenda for social constructivists. This agenda reflects the importance of studying forms of understanding that precede explicit comprehension indexed by theory-of-mind tasks (sect. 5). Forms of social understanding especially pertinent to the prelinguistic child concern the communicative purpose of a word rather than figuring out, through inference and introspection, a preverbal mentalistic referent. Consequently, investigators should find that these pragmatic ends are evident to the prelinguistic child. For example, if "want" is used to express obtainment, then the linguistic stimulus ("the [actor] wants [that target]") should be associated by infants with scenes where an actor is pulling a target object toward herself rather than scenes where the actor is pushing away the target. Verb comprehension would be evident in this test when children look longer at the scene matching the linguistic stimulus (see Hirsh-Pasek \& Golinkoff 1996). Experimental tasks of this nature point the way toward illuminating the interpersonal and social features from which mental concepts originate. And this is a central goal for social constructivist theories of social understanding.

\section{Toward a collaborative community of minds}

\section{Katherine Nelson}

Department of Developmental Psychology, City University of New York Graduate Center, New York, NY 10016. knelson@gc.cuny.edu

Abstract: Three points extend the authors' comprehensive and provocative argument: (1) The idea of "entering a community of minds" is suggested to replace theory of mind or social understanding; (2) learning words and concepts through a Wittgensteinian process often involves a period of "use without meaning"; (3) concepts based in social interaction are achieved through collaborative - neither individual nor social alone - construction.

Carpendale \& Lewis $(\mathrm{C} \& \mathrm{~L})$ have done the field a great service in assembling the theory and evidence for a social interaction account of the development of social (and psychological) understanding in infancy and early childhood. In doing so, they have also clarified the distinction between an enculturation model of development and a constructivist model based in triadic relationships integrating individual and social dimensions.

This approach is broadly consistent with proposals and arguments that I, and my students, have made (Nelson 1996; Nelson, in press; Nelson et al. 1998; 2000; 2003; Nelson \& Shaw 2002). From this perspective, I suggest that they present an excellent case but do not go far enough in a cultural-historical direction. This is not to endorse a radical relativism with regard to concepts of mind but rather to emphasize that a child's development of such concepts takes place within sociocultural-historical contexts. These contexts extend beyond triadic relationships to those embracing many community members who display increasingly various mental perspectives and attitudes toward matters of concern to the community.

Based on these concerns, we propose reconceptualizing "acquiring a theory of mind" as the process of entering a community of minds. This construct emphasizes the notion of a gradual approach toward the status of member of the community wherein matters of minds are of interest and concern and are topics of talk. These concerns involve plural minds, indicating that the problem for the child, as for people in general, is not just to track and coordinate the perspectives of the self and one other, but to embrace the idea that people in general have different knowledge about and perspectives on states of the shared world.

The problem of integrating early social understanding and interaction with the acquisition of language is viewed by the authors from a Wittgensteinian perspective of language as activity. They reject the prevailing view that concepts are acquired before words can be learned to express them, arguing instead that children learn concepts and words at the same time. Their brief discussion gives little hint of the radical position they are taking on language learning in relation to current conceptions of the process. Nonetheless, their view accords with the evidence not only from theory of mind but from evidence of word learning in natural environments, as they note in citing Nelson (1997). Further, a study of the acquisition of the words know and think by $2 \frac{1}{2}$-to-4-year-olds in natural home contexts was reported in Nelson and Shaw (2002; Shaw 1999). There we showed that, consistent with the claims of the target article, these words were used casually by parents in activity contexts (playing, eating) most frequently to direct the interaction, with fewer uses referring to mental states. Most of the children of the ages studied knew and used the words in activity contexts consistent with the uses by their parents, but their reference to mental states was ambiguous at best, with little evidence of shared meaning for these terms, despite the shared usages.

The evidence supports the authors' claim that words and concepts are acquired together; I would add the caution that words are often "used without meaning" by the child for a long period (months or years) during which the concept remains partial or elusive. The criteria for the use of mental state words are difficult for a learner to discern for two reasons: on the one hand, the mental 
state concept (e.g., know or think) does not exist a priori in the child's mind and can only be inferred from opaque references to other words; and, on the other hand, the words themselves have many different uses in activity contexts, only some of which refer to mental states. Consider the mother saying "I think this one goes here," as she places a puzzle piece; in what way might a child differentiate this from the statement "this one goes here?" (The ambiguous meaning of think in everyday contexts and psychological tasks is discussed in Nelson et al. 2003.) These comments bear on the authors' research agenda for the study of the development of "shared meaning" (Nelson 1985), which they point out is one of the most difficult and important problems (and, I would add, most neglected) in developmental psychology.

The authors make an important point that children must assume that they live in "a common, stable, external world that is the same for the self and others." But as they note, this assumption is challenged when through language children learn that other people's experience is different. Coming to grips with this knowledge is the entry point to the community of minds and the foundation for the radical change in self and consciousness that takes place during the later preschool years, of which the standard theory-ofmind tasks have tapped one small piece. This knowledge depends upon evidence derived in social interactions but it does not come as a prepackaged concept; rather it requires knowledge construction from accumulating pragmatic evidence. At the same time it is a mistake, I believe, to suggest that the construction is achieved by the individual child, any more than to suggest that it is transmitted as a social construction from parent to child. Rather, the process may best be viewed as a collaborative construction in which the child's emerging awareness of different experiences and different perspectives is supported, explained, and elaborated by parents or others through everyday discourse.

One of the most important kinds of such discourse is talk about the past and the future - reconstructing memories of child and parent and forecasting coming activities. As recent work in the emergence of autobiographical memory has shown, more elaborative talk about the past by mothers is associated with more and earlier personal memories as well as better performance on theory-of-mind tasks by their preschool children (Reese 2002). This is but one indication that theory of mind is not a separate or modular achievement, but rather, one of an integrated complex of developmental moves taking place during the later preschool years, which bring the child to a new level of social and psychological understanding, preparatory to the further developments that will occur later during middle childhood.

\section{Children's understanding of mind: Constructivist but theory-like}

\section{Ted Ruffman}

Department of Psychology, University of Otago, Box 56, Dunedin, New Zealand.tedr@psy.otago.ac.nz

\begin{abstract}
Although in general agreement with Carpendale \& Lewis's (C\&L's) claims, I argue that (1) gradual development is better supported by within-task eye gaze/verbal comparisons; (2) gradual development and social construction do not contradict the theory-theory view; (3) there is good evidence for an early developing self-other distinction; and (4) the language-false belief link could be mediated by parental talk.
\end{abstract}

Carpendale \& Lewis (C\&L) argue against the notion that theory of mind is innate. They might concede, however, that there is likely to be some innate basis for theory of mind, such as newborn infants' interest in the eyes and face (Bakti et al. 2000; Johnson \& Morton 1991). This interest means that infants' attention is focused on a region that expresses mental states so that they are ideally situated to learn about theory of mind either through their own initiative (e.g., by asking others questions about motivations for actions; Dunn 1988), or through others' initiative (e.g., through parent teaching or siblings' and peers' influence in play and elsewhere). C\&L do a good job of discussing evidence in favor of this latter path and, in so doing, do the field a service.

C\&L justify their claims about gradual development using contrasting results obtained with different false belief tasks. Yet differences in information-processing demands or in the concept actually tapped in different tasks weaken such arguments (Perner et al. 1994). A stronger case for gradualism might be made on the basis of within-task discrepancies (Clements \& Perner 1994; Garnham \& Perner 2001 Garnham \& Ruffman 2001; Ruffman et al. 2001b). For instance, we found that children who passed an eyegaze measure of a false belief task (looking correctly when anticipating a story character's return), but gave an incorrect verbal prediction, could be split into two groups (Ruffman et al. 2001a). The younger such children showed complete confidence in their verbal answer, betting all counters (used to indicate the character's predicted location of return) on the location consistent with their verbal answer. In contrast, some of the older children with correct eye gaze but incorrect verbal performance showed awareness of the knowledge manifest in their eye gaze by placing at least some counters on the location consistent with their eye gaze. Thus, confidence varied for children who showed identical performance on both the eye-gaze and verbal measures.

Likewise, older children who passed both the eye-gaze and verbal measures were not fully confident in their verbal answer, because they placed at least some counters on the location that was inconsistent with both their eye gaze and verbal answer. In sum, changes in eye gaze and confidence over time are consistent with the following pattern of gradual development: (1) children fail both eye-gaze and verbal measures (no understanding evident); (2) children pass the eye-gaze but not the verbal measure, and show full confidence in their verbal response (understanding is implicit); (3) children pass the eye-gaze but not the verbal measure, and lack confidence in their verbal response (the dawning of conscious insight into false belief); (4) children pass both measures but still lack full confidence in their verbal response; (5) children pass both measures and are confident of their verbal response. Increasing confidence seems to indicate a deepening understanding, perhaps because children come to understand better why the character will hold a false belief.

Although I agree that theory-of-mind knowledge is constructed in a social context and understanding is gradual, I do not see either conclusion as a threat to the theory-theory (TT). TT is based on the idea that children form theories about the mind that are in some ways similar to scientific theories. Scientific theories are often the result of years or decades of hard work, sometimes with many scientists from different laboratories contributing. Hence, scientific theories, the very basis for TT, are typically constructed both gradually and as the result of a community (social) effort. Arguments against TT on these grounds are therefore erroneous. Instead, arguments should focus on whether the structure of children's understanding is theory-like (i.e., possesses the characteristics of a theory), and there are good reasons for thinking that this is at least partially true (e.g., Gopnik \& Wellman 1992; Perner 1991; Ruffman 1996; Wellman 1990).

There are other more minor issues. First, although C\&L restrict their criticisms of TT to false belief understanding, in a broader context current versions of TT do allow for gradualism. For example, it is a basic tenet of TT that desire understanding begins before belief understanding, which begins before belief-based desire understanding, and so on. Second, C\&L argue against the simulationist idea that children understand the mind using analogy, claiming that this presupposes a distinction between self and other. Yet, infants understand something about others' desires as different from their own by 18 months of age (Repacholi \& Gopnik 1997), and mirror self-recognition indicates some rudimentary understanding of self by 3 or 4 months of age (Rochat \& Striano 2002). This allows a role for individual development of social understanding from some time in early or late infancy at least. Fur- 
ther, there are also non-introspectionist versions of simulation theory (e.g., Gordon 1992).

Finally, the authors note the robust correlation between social understanding and language ability. One caveat is that this relation might hold only for explicit performance on theory-of-mind tasks. Preliminary evidence indicates that language might not correlate with implicit understanding (Ruffman 2000). In addition, although some might take the language-social understanding relation as evidence for individual, nonsocial factors affecting theoryof-mind development, there is a way of reconciling this relation with the social constructivist view. Mother mental state language is highly related to (1) child mental state language (e.g., Brown \& Dunn 1992; Ruffman et al. 2002); and (2) child mental state understanding (e.g., Dunn et al. 1991b; Ruffman et al. 2002). In addition, aspects of mother language (e.g., question asking) are related to later aspects of children's expressive, syntactic language and vocabulary (Hoff-Ginsberg 1986; Hoff-Ginsberg \& Shatz 1982; Weizman \& Snow 2001). It is entirely possible that the link between child language and theory of mind would be at least partially mediated through parents' linguistic input (e.g., mother language facilitates child general language which facilitates child theory of mind).

\section{Wittgensteinian developmental investigations}

\section{John Shotter}

Department of Communication, University of New Hampshire, Durham, NH 03824-3586.jds@hypatia.unh.edu http://pubpages.unh.edu/ jds

\begin{abstract}
I criticize Carpendale \& Lewis's (C\&L) attempt to produce a Wittgensteinian theory, as an alternative to work in the "theory of mind" tradition, not because I disagree with it as theory, but because Wittgenstein would be critical of any attempt to make such a use of his work. His concern is with descriptions, not theories.
\end{abstract}

Carpendale \& Lewis (C\&L) want to criticize the whole "theory of mind" tradition in developmental research for its grounding in "individualistic processes." Instead, they want to propose an "alternative theory" drawing on, among others, Vygotsky and Wittgenstein, but especially on "Wittgenstein's arguments." I wholeheartedly endorse their turn to Wittgenstein. However, I am still critical of their use of material from Wittgenstein's later philosophy. For, after all, in the Investigations he notes with respect to his methods of inquiry that:

It was true to say our considerations could not be scientific ones . . . we may not advance any kind of theory. There must not be anything hypothetical in our considerations. We must do away with all explanation, and description alone must take its place. (Wittgenstein 1953/1968, No. 109). ${ }^{1}$

His argumentative and other kinds of remarks are aimed at a quite different kind of investigation from those of a scientific kind.

Although Wittgenstein is not critical of science as such (in its own, proper context), the whole scientific approach is in fact inimical to the character of his investigations. His investigations are of a grammatical kind. Wittgenstein's remarks are thus not at all aimed at arguing for what is in fact the case. They are to do with "giving prominence to distinctions which our ordinary forms of language easily make us overlook" (No.132), with drawing our attention to "what is possible before all new discoveries and inventions" (1953/1968, No.126) - they are expressions of a concern with what already lies "seen but unnoticed" (Garfinkel 1967, p. 36) in the background to all our everyday (and professional) communicative activities. Although each of us might uniquely do our own thing - like taking our own particular path through a landscape if we are not to mislead or confuse those around us, they must be able to see how the path we are taking relates to those possible for them; if they are to coordinate their activities with ours, they need to know, not what we are actually doing now, but its "point," what it is aimed at in the future, where we are trying to get to; they must be able to "follow" us. Whereas in scientific investigations, "we feel as if we [have] to penetrate phenomena," says Wittgenstein (1953/1968), his grammatical investigations are "directed not towards phenomena, but, as one might say, towards the "possibilities' of phenomena” (No.90). Hence, theories (and arguments in their support) would be necessary in these investigations only if one were convinced that the influences shaping people's behavior in this grammatical fashion were so radically hidden that they could be discovered only indirectly, by a process of scientific investigation. Whereas, as Wittgenstein (1953/1968) notes: "If it is asked: 'How do sentences manage to represent?' - the answer might be: 'Don't you know? You certainly see it, when you use them.' For nothing is concealed" (No. 435). Indeed, they cannot be concealed, else all around us would have to orient toward us as aliens from another planet.

In other words, like C\&L, Wittgenstein sees all the events of importance in our teaching our children to be like ourselves (as well as in our coming to an understanding of each other's unique "inner lives") as occurring "out there" in the living relations between ourselves and the others and othernesses around us. But, as Wittgenstein (1953/1968) realizes, the relevant events are of such a subtle and complex kind, and "it all goes by so quick" (No. 435), that we cannot easily get an overall view of them. A visual grasp allowing us to survey all their detailed interconnections at once hence, to know ahead of time what might follow from what seems, at first, impossible.

It is at this point, however, that Wittgenstein and C\&L part company. For what C\&L miss, as indeed the whole tradition of "scientific" inquiry in psychology misses, is the fact that certain socially shared influences, influences that Wittgenstein calls "grammatical" influences, are always ineradicably at work between us in our use of language. Although we easily fail to notice them because of their socially distributed nature, it is the undeniable fact that these influences are always present in our meetings with each other which he wants to bring to our attention. The meanings of the words we use in our utterances are not, and never can be, a matter of our own choosing.

Because the events relevant to our instructing our children and understanding each other's "inner lives" are not in fact radically hidden, Wittgenstein does not turn to theoretical claims and conjectures in their investigation. This is where his later philosophy is quite revolutionary. He introduces a whole compendium of devices - vignettes, dialogues with other "voices," arguments, dramatic scenes, metaphors and similes, striking examples, subtle particularities, and so on - all aimed, not at learning "anything new," but at "understanding something that is already in plain view ... something that we need to remind ourselves of" (No. 89).

In practice, then, Wittgensteinian investigations into child development would not involve researchers in continually arguing for theories, either in terms of evidence derived from attempts to test them empirically, or conceptually in terms of whether they adequately encompass all the relevant phenomena or not. They would face a different kind of task. Just as we come to know our "way about" inside a particular new house or city by taking the trouble to explore connections between its unique details to gain a sense of what leads to what, so we can gradually develop the same kind of clear understanding of what is involved in our children coming to an understanding of others' minds. And to be confident in this way, we do not feel that we need to be able to write out the whole town map. For Wittgenstein wants in his investigations "to replace wild conjectures and explanations by the quiet weighing of linguistic facts" (1981b, No. 447), thus to produce merely a description of the facts that matter in the issue concerned - a description which, if one was initially intellectually disoriented, ${ }^{2}$ justifies saying to those around one (at least for the immediate, practical purposes in hand): "Now I know how to go on" (1953/1968, No.154). C\&L take Wittgenstein’s philosophy piecemeal; it needs to be taken as a whole. 
Commentary/Carpendale \& Lewis: Constructing an understanding of mind

NOTES

1. All date-only citations are to Wittgenstein's works.

2. "A philosophical problem has the form: 'I don't know my way about" (1953/1968, No.123).

\section{Social understanding and the cognitive architecture of theory of mind}

\author{
Michael Siegal \\ Department of Psychology, University of Sheffield, Sheffield S10 2TP, United \\ Kingdom.M.Siegal@sheffield.ac.uk \\ http://www.shef.ac.uk/psychology/staff/siegal.html
}

Abstract: Although Carpendale \& Lewis (C\&L) correctly emphasize the importance of conversation in children's social understanding, they neglect several complex issues. Contrary to their assertion, the focus on mental state processing has not been misplaced, and there is a need to recognize that different aspects of social understanding are liable to undergo distinctive developmental changes that vary in relation to social interaction.

Carpendale \& Lewis's (C\&L's) article is a welcome addition to the debate on the relation between language and theory-of-mind reasoning that has been stimulated in two recent related BBS target articles (Bloom 2001; Carruthers, in press). According to C\&L, "A common problem with the dominant perspectives of the field is that each focuses on the cognitive architecture of mental state reasoning, without reflecting on the social landscape in which such reasoning is constructed" (target article, sect. 2.2, last para.). The main motivation for the social interaction approach that C\&L propose is the observation that social understanding develops gradually and that research on social understanding is overly fixated on theory-of-mind false belief tasks that prevent us "from examining the longer view of development" (sect. 4.1, penultimate para.). In their proposal, C\&L rightly highlight the central role of conversation in development. However, they do not adequately recognize that this role varies according to different aspects of social understanding, and they gloss over the fundamental distinction between having the concept of belief and differences in how specific beliefs are used in judging persons and situations (Scholl \& Leslie 1999).

It is no wonder that so much attention has been fixed on the core cognitive architecture of theory-of-mind (TOM) reasoning that involves knowledge of how mental states such as beliefs may not conform to reality. Correlations between performance on TOM tasks and opportunities for positive social interaction from peers and siblings are consistent with the notions that forms of social interaction speed up the manifestation of TOM reasoning and that having TOM may be a good thing for a wider social understanding (Peterson \& Siegal 2002). However, as shown on tasks involving predictions of the behavior of a protagonist who holds a false belief, TOM is achieved by all typically developing children by about four to five years of age. Modifications to the structure of these tasks in order to ensure that children understand the relevance and purpose of an experimenter's questions reveal competence at an earlier age (Siegal 1997), and, to a considerable extent, the tasks themselves really amount to tests of children's conversational understanding (Bloom \& German 2000). Early immersion in conversation with others may suffice to trigger the display of TOM reasoning even in three-year-olds, alerting children to the fact that others are repositories of information about mental states that may differ from one's own, and from reality.

But obstacles to conversational understanding and hence TOM reasoning can occur. Conditions such as deafness, autism, and anarthria often do not permit the child to engage even minimally in conversations that permit insight into the nature of mental states. In all these cases, children may function quite normally or even excel in situations that involve reasoning about number, biology, or physics and yet have protracted difficulty on TOM tasks. This pattern of results is of great significance to developmental psychologists and cognitive neuroscientists, as it points to the possibility of early auditory and attentional barriers that preclude participation in conversations and success on TOM tasks (Siegal \& Varley 2002). The diagnosis of such barriers promises to alleviate the social isolation of children with developmental disorders, in the process enhancing their communication and literacy. A neurocognitive approach is fundamental to the study of this aspect of social understanding.

Unlike the concept of belief, specific beliefs do vary in typically developing children. In particular, children may vary in their specific beliefs about the usefulness of false belief knowledge in answering questions about what they and others know. C\&L cite a study by Varouxaki and colleagues (1999) suggesting that many (but not all) five-year-olds neglect to report knowledge that can be inferred or deny that they are ignorant despite a lack of knowledge. They interpret these responses to reflect the development of beliefs beyond those shown on TOM false beliefs tasks. Yet, in this instance, forces of enculturation and language may either render some children to be more modest than others in their interpretation of knowledge (Lee et al. 1997), or prompt children to give affirmative or other perseverative responses in situations in which they do not yet understand the purpose and relevance for the task at hand (Deák et al. 2003; Fritzley \& Lee 2003). Such beliefs involve an altogether different aspect of social understanding from that of simple TOM reasoning - one that does need to be considered on its own merits in terms of social interaction influences.

Therefore, whereas the expression of TOM reasoning itself can be viewed in terms of a "poverty of the stimulus" analysis in that, like the syntax of language, only a minimal environmental input seems to be needed for it to emerge, social interaction can powerfully influence specific beliefs about the knowledge, emotions, and intentions of others. Gradually, the massive cultural differences in adult beliefs come to be reflected in children's beliefs (Hejmadi et al., in press; Shweder et al. 1998) - a development that is distinctive from the core cognitive architecture of TOM.

Can differences in specific beliefs be explained solely through the Piagetian constructivist processes that C\&L advocate? It is likely that different aspects of social understanding undergo distinctive developmental changes, much as does development in various scientific domains such as biology, cosmology, and physics (Siegal 2002). For example, in reasoning about food, particularly the edible-inedible distinction that is close to survival, children are constrained to initiate conversations in order to meet the sharply defined goal of avoiding contamination. By contrast, no such conversations are necessarily forthcoming on cosmological knowledge. For children to know about the shape of the earth and the day-night cycle may require direct cultural transmission in school. A constructivist account does not fully characterize either of these changes. Similarly, the landscape of social understanding is huge. It includes the interpretation of facial expressions and the acquisition of cultural traditions of dietary laws and other social customs. We await an analysis dedicated to how children's understanding of such varied aspects of the social world comes about.

\section{Acts of judgment, not epistemic triangles}

\section{Leslie Smith}

Department of Educational Research, Lancaster University, Lancaster LA1 4YL, United Kingdom.1.smith@lancaster.ac.uk http://www.lancs.ac.uk/staff/erals/

Abstract: Carpendale \& Lewis's (C\&L's) reanalysis of Chapman's (1999) epistemic triangle dealing with the coordination of interactions with physical objects and people's communication is misleadingly incomplete. An alternative proposal is outlined combining the causality of action with the normativity of knowledge in acts of judgment. This alternative is empirical and developmental, with a focus on rich but neglected phenomena. 
Carpendale \& Lewis (C\&L) argue for a third alternative to individualism and collectivism with regard to cognitive development in terms of Chapman's (1999) epistemic triangle (ET). This triangle has a central apex in the coordination of the remaining duality consisting in interactions with physical objects and communications with other people (target article, sect. 3). My argument is that C\&L's re-analysis is misleadingly incomplete. My alternative proposal is in terms of the human capacity to make judgments.

What is an object? An answer in terms of Popperian realism about three worlds, recently recast by Bereiter (2001), is instructive: world 1 is the world of physics; world 2, the world of psychology/sociology; and world 3, the world of epistemology. Translated into C\&L's re-analysis, an ET is the coordination of world 1 physical objects with minds in societies in world 2 . But this is problematic. The objects and properties of these worlds are exclusive, and so problems of Kuhnian incommensurability remain. First, physical objects and their properties are not psychosocial. Nor are psychosocial objects and properties physical. This means that they have in common no distinctive properties - other than causality, which is addressed below - and so C\&L's re-analysis has not been carried through. Second, there is worse: Whereas physical and psychosocial objects have causal properties, world 3 epistemic coordination objects would have normative properties. Paradigm cases of normativity include truth-values (only truths can be known) and entailments (knowing $3+4=7$ entails $3 \times 4=12$ ). Nor are these the only cases in the class (Smith 2002). There is nothing in C\&L's proposal to show how this reconciliation of the normative and causal properties of knowing could be carried through in the construction of true knowledge bound by necessitation. Hence, ET coordination in C\&L's re-analysis names but does not explain cognitive development.

An alternative proposal is to regard objects intentionally as the content of acts of judgment (Smith 2002; 2003). Acts include physical and communicative interactions, and so straddle worlds 1 and 2 . These interactions occur as lawful regularities in contingencies, contexts, and cultures for causal explanation in psychology/sociology. An important type of act is assertion and denial when an agent makes a judgment. The content of a judgment is an intentional object based on norms internal to the act. Norms include rules, obligations, and directives with a common logic (von Wright 1963). They occur in all domains of knowledge and are used by individuals in societies. Acts have agents who regulate their actions in terms of norms - following Piaget (1965a, p. 159) "a subject is always 'normed." The implication is not whether agents use norms, but rather which norms these are and how they are used. Regulations may occur as normative facts which are "imperative rules whose origin is in social interactions of all kinds, and which act causally, in their turn, in the context of individual interactions" (Piaget $1977 / 1995$, p. 69). Normative facts are facts and are empirical. They are open to investigation at all developmental levels. Central to this developmental epistemology (DE) is the proposal that (intentional) objects are constructed in virtue of linkages between causal facts and normative facts through uses of the capacity to judge.

Here are some examples of normativity covering both adults and children:

A. Martin Luther was directed at a religious tribunal to explain why his judgment was to be trusted over that of his peers. Luther argued that "I do not accept the authority of popes and councils, for they have contradicted each other. Here I stand, I cannot do otherwise."

B. Galileo argued that the Ptolemaic and Copernican models of the universe were false and true, respectively. He was directed by the Church to accept that this analysis was erroneous. Asked to explain why he had violated this command, Galileo insisted that he had no memory of agreeing to it.

In examples $[\mathrm{A}]$ and $[\mathrm{B}]$, an individual is in social dispute with peers. This dispute is manifest in incompatible judgments, which are due to commitments to divergent norms in their societies.

C. Mat was asked to add 3/4 and 1/4, adding numerators and denominators, making $4 / 8$, and then through a pie chart, making 1. Asked a normative question about how to decide which answer was right, Mat replied permissively: "it depends on which method you are told to use" (Kamii 1982).

D. Normative commitments about number conservation were at work in $20 \%$ of children's incorrect responses: lengthening one line of counters reduced their number in that "you've taken two away (and so) these two aren't there." These judgments were analogous to a normative disqualification in a game when a player is "sent off" (Smith 2002).

In [C] and [D], children are in causal settings influencing their performances. Their erroneous judgments are made by reference to norms which are divergent from those of their teachers.

E. In a study of mathematical induction, young children repeatedly added one counter to each of two containers, where initially X's contents were one more than Y's. Asked a generalisation question, John replied "that $(\mathrm{X})$ would be right up to the cover in the sky and that $(\mathrm{Y})$ would be right up to God, so then they would still have to be more." This was superb reasoning by analogy through a cultural belief that God lives in Heaven on the top of which was a cover. Thus were the contents of B still more than those of A, and necessarily so (Smith 2002).

Cases $[\mathrm{A}]$ and $[\mathrm{B}]$ show that normative advances are made by adults, and [E] that they are made by children, with [C] and [D] giving testimony to the difficulties. These rich phenomena cry out for explanation. Central to DE is how "each individual is led to think and re-think the system of collective notions (Piaget 1977/ 1995 , p. 76). Norms are used in the initial "thinking" of sociocultural notions, and are developed in their "rethinking." Key advances are made from causality to normativity (Piaget 1977/1995, p. 51), from "normative pressure" to autonomous normativity (von Wright 1963). Quite how such advances could be made remains indeterminate in C\&L's re-analysis.

\section{A penny is your thoughts? Reflections on a Wittgensteinian proposal}

\author{
Bryan W. Sokola and Christopher E. Lalonde \\ a Department of Psychology, Simon Fraser University, Burnaby, British \\ Columbia V5A 1 S6 Canada; ${ }^{\mathrm{b}}$ Department of Psychology, University of \\ Victoria, STN CSC, Victoria, British Columbia V8W 3P5, Canada. \\ bryan_sokol@sfu.ca lalonde@uvic.ca http://www.psyc.sfu.ca \\ http://www.uvic.ca/psyc
}

Abstract: Although in fundamental agreement with Carpendale \& Lewis's (C\&L's) position, we discuss a potential source of confusion regarding the socially constituted nature of mental states. Drawing from recent work by Kusch (1997; 1999), we argue, more specifically, that mental states are instances of "artificial kinds," and so, stand between the more common classificatory extremes of "the natural" and "the social."

Most of us, we suspect, labor under the impression that our thoughts are private and that even if Big Brother scrutinizes other aspects of our lives, at least our mental lives are safe from prying eyes. To be told otherwise - that is, to hear on good authority that our minds are not the private sanctuaries we have always imagined them to be - would be unsettling. Although this was not our own first reaction to Carpendale \& Lewis's (C\&L's) broad proposal regarding the socially constructed nature of the mind, we argue here that perhaps it should have been. In their treatment of the debate concerning the relative contribution of social versus individual processes in development, C\&L effectively "out" the often closeted "individualistic" assumptions underlying much of the presentday smart talk about children's understanding of mind and, in the bargain, usher in a set of perhaps even more radical claims. That is, Orwellian threats notwithstanding, we suggest something even more insidious is afoot in C\&L's proposal, not the least of which is that our mental lives may never be quite so "private" again. 
Perhaps one of the more controversial claims that C\&L make in this regard turns on the so-called "contents" of the mind (mental states such as beliefs and intentions) and their relation to human action. In rejecting the "causal psychological view of the mind" that posits mental states as hidden causal "entities" driving behavior, C\&L effectively claim that our language about mental states has fooled us all and that, in fact, "there are no such contents." All of this seems quite hard to swallow. Nevertheless, C\&L's position is not without support. Although borrowing ostensibly from Wittgenstein to develop their alternative view, C\&L might just as easily have taken a page from Dewey (see, e.g., his 1912 essay, "What are states of mind?" in Dewey 1912/1979), who similarly argued that "psychical" states are the result of "retrospectively" reframing our broader activities and experiences - what he calls "organic reactions" - and, as such, "are neither antecedents nor concomitants, in a separate realm of existence . . . but are the very qualities of these reactions" (Dewey 1912/1979, p. 36). The upshot of this view, as expressed in more current philosophical circles, is that "our psychological classifications are constitutive of our mental states and events" (Kusch 1997, p. 18; see also Taylor 1985), or, phrased more polemically, that our private thoughts are in fact "social institutions" (see Kusch 1999, pp. 321-68).

Much of what is polemical here, however, follows from a somewhat different classification issue. The culprit in this case is the traditional bimodal scheme of classifying things as either natural or social kinds. As the logic in this scheme would have it, if natural kinds refer to real things in the world, then, by default, social kinds must refer to made-up things, or, worse, to nothing at all. Mental states, in this either-or classificatory system, must either be seen then to somehow cut the mind-brain at its natural joints or amount to mere "mythical posits." C\&L, as well as many others who might otherwise agree with their assessment, are likely to be dissatisfied with these two options. Thankfully, there are other, more rewarding ways to divide the spoils.

In addition to - or more precisely, in between - such natural and social kinds are what some philosophers have come to call "human" (Hacking 1992) or "artificial” (Kusch 1997; 1999) kinds. To be clear, insofar as each kind involves a self-referential component, they are all in some sense socially constructed. Still, the degree of self-referentiality differs in important ways for each. At one end of this continuum, there are social kinds that are entirely created, sustained, and enforced by our collective actions without making any kind of reference beyond such activity. That is, they admit no "alter-reference" that, as Kusch (1997) explains, "refers away from itself toward individuals in the physical world, individuals that exist independently of the reference" (p. 17). The other anchor point - natural kinds like mountains and rivers - possesses these independent characteristics, although even here some collective agreement is necessary in order to establish the criteria by which we meaningfully sort them. Finally, and falling in between these extremes, there are artificial or human kinds that possess such an alter-reference, much like natural kinds, but that are also similar to their social counterparts in that they do not exist apart from human classifying and meaning-making activities - in fact, human activities are what bring them into physical existence in the first place.

Importantly, then, artificial kinds are no less real than any other humanly constructed or manufactured object. More central to our purposes here, however, is not so much what they are, but what they sometimes become. That is, artificial or human kinds are sometimes prone to a reification process by which the constructive, or socially constituted, element is overlooked or even forgotten. Kusch (1997) claims that this is the case, for instance, with money: "'to be money' is easily thought of as being an intrinsic, non-social property of certain metal discs" (p. 3). Although it would hardly seem to require a philosopher to demonstrate that this is a mistake, a related error is often made when it comes to understanding mental states. Like money, mental states are an instance of an artificial or human kind, and not coincidentally, are "easily thought of as being intrinsic, non-social properties of certain entities called selves or minds" (Kusch 1997, p. 3).

Viewing mental states as human or artificial kinds (rather than natural or social), and acknowledging this tendency toward reification, clearly fits with the Wittgensteinian proposal on offer by $C \& L$ and, we argue, helps to further bridge what C\&L call "the impasse between individual and social perspectives on social understanding" (sect. 5, para. 1). It does so, we claim (and here is our main point), without at the same time drawing us toward the enculturation view that $C \& L$ rightly warn us against, and without whittling away at the contribution of individual agency in the construction of mental life.

\section{The social matrix reloaded: An attachment perspective on Carpendale \& Lewis}

\section{Howard Steele \\ Attachment Research Unit, Sub-Department of Clinical Health Psychology, University College London, London WC1E 6BT, United Kingdom. h.steele@ucl.ac.uk}

Abstract: The "new" theory of Carpendale \& Lewis (C\&L) needs be compared with existing elaborated and tested models concerning the social origins underpinning the sense of being a person with thoughts and feelings in relation to others. Illustrations are provided from contemporary attachment theory and research in the context of questioning the potential legacy of Piaget as a theorist of social relationships.

Carpendale \& Lewis (C\&L) are right to draw attention to the primacy of social context, for our sense of self depends on the meanings we take from, and give to, our closest relationships. The view advanced by C\&L is highly compatible with elements of attachment theory (Ainsworth et al. 1978; Bowlby 1969/2000). Bowlby regarded his theory as one among a range of psychoanalytic object-relations theories (Bretherton 1998). Object-relations theories have in common the view that the primary motivation in human life is the wish to form and maintain an enduring emotional relationship with other persons (Steele \& Steele 1999).

The complicated interactive dances that typify mother-andbaby interactions are thought to facilitate or dampen the infant's regulatory system and brain development (Schore 2000). As Tronick and Weinberg (1997) have described, "mutual regulation is one of the processes that shapes the human brain itself ... Thus the brain, like emotional experience, is jointly created" (p. 73). What infants learn from these early social interactions is thought to be stored in their internal working models, which denote an active person experiencing and constructing emotions, expectations, memories, and narratives (Nelson 1999).

C\&L remind us that Piaget had much to say about the fundamental role of social relationships upon cognition. Piaget's distinction between constraining and cooperative relationships captures some of the risks and opportunities of social interaction. Yet this dichotomous model leaves us a bit short, as it does not take into account much of the nuances in describing the complexities of human relationships. Contemporary attachment theory and research, such as those utilising narrative analyses in children and adults (Main et al. 1985), pay close attention to an extensive range of identifiable speech patterns concerning attachment topics such as separation, rejection, loss, and trauma. Some of these speech patterns, such as profound lapses in the monitoring of speech or reason concerning past loss or trauma, are markers of risk factors for both parent and child (Steele \& Steele 2003; van IJzendoorn \& Bakersman-Kranenburg 1996; Wallis \& Steele 2001). Other of these speech patterns, sharing a robust adherence to Grice's (1975) maxims of "good conversation," that is, truth, economy, relation, and manner, are predictors of optimal parenting and emotional well-being in children (Steele 2002). 
The theory of theory of mind advanced by Carpendale \& Lewis could thus be bolstered by incorporating the burgeoning knowledge on the nature of parent-child interactions and on individual variations in dyadic emotion-regulation patterns out of which emerges a sense of self. Recent theorising and research on infant development underscores how early and in what contexts the sense of agency and relatedness may be observed to thrive or suffer (Koulomzin et al, 2002; Schore 1994; Stern 1985; Trevarthen 2003; Tronick \& Weinberg 1997). Further data from diverse sources, such as facial affect recognition (Skuse 2003), are converging to elucidate a more detailed understanding of emotional development.

One would wish to heed the sympathetic call by C\&L to take account of the infant's social context, dyadic, triadic, and beyond. However, the extent to which this is a new call or an old echo is debatable. Consider the continued relevance of Bronfenbrenner (1979) or psychoanalytic object-relations theorists. Beyond Bowlby, the words of Donald Winnicott come to mind: "there is no such thing as a baby." This provocative statement draws immediate attention to the baby's social context. At the same time, Winnicott did not underestimate the paradoxical - both individualistic and social - challenge of development. Healthy psychological development, he urged, is likely to be secured by cultivating and protecting the capacity to be alone in the presence of another (Winnicott 1965).

C\&L find support for their approach in the findings that "secure" attachments appear to facilitate the development of a theory of mind. In our own longitudinal attachment research (Steele et al. 1996), we have also observed advanced theory-of-mind skills not only among infants with a history of a secure attachment, but also among those with a previously observed highly anxious/fearful, disorganised attachment to mother (Fonagy et al. 1997). Notably, these successful predictions from infant-mother attachment security at one year to theory-of-mind performance at age five were in respect of belief-desire reasoning skills, that is, where the child was required to guess correctly the feeling state of a deceived puppet. Attachment security did not predict belief-belief reasoning, that is, where the child was required to guess correctly the behaviour of a doll acting on information that is no longer valid.

Thus, the relations between infants' social experiences and the evolution of their theory-of-mind skills are likely to depend on the extent to which the context loads more on the social-emotional register as opposed to the cognitive-behavioural one. Also, given the similar performance we have observed in children with organised-secure and disorganised early attachments, we must not assume that similar phenotypic outcomes share the same type of social determinants. In one case a child may be advanced in theorising about emotion because one or both parents have provided much helpful talk about feelings (Dunn et al. 1991a). In another case, the child may be advanced because the parent was liable to unpredictable and frightening behaviour such that the child needed to know when to run or hide. The value of quickly detecting (on the caregiver's face) the imminent rise of anger before it reaches its full-blown potential (when this has previously led to abusive behaviour from the caregiver) cannot be underestimated (see Pollak \& Sinha 2002).

Hence, the long-term effects of early social experience are likely to be manifest in the domain of emotion recognition and emotion understanding (Steele et al. 1999) and social cognition (Steele et al. 2002) and not necessarily in the broad cognitive domain, to which most theory-of-mind tasks belong. In other words, a social constructionist account of social cognition may be highly apt, but an individual-differences and emotion-focused account of many aspects of cognition may nonetheless have continued relevance.

\section{The internalization of mental state discourse contributes to social understanding}

\author{
Douglas K. Symons
}

Psychology Department, Acadia University, Wolfville, Nova Scotia B4P 2R6, Canada.doug.symons@acadiau.ca

http://ace.acadiau.ca/science/psyc/dsymons/

Abstract: Children's exposure to and participation in mental state discourse contributes to their development of social understanding. Vygotsky's mechanism of internalization is used to account for this process, which has advantages of cultural and linguistic universality. If children internalize mental state discourse, however, then their own use of mental state language should be related to social understanding.

Carpendale \& Lewis (C\&L) are commended for their social constructivist account of the origins of social understanding. They provide a theoretical context for recent work which has shown that various features of the early social environment of children are related to their concurrent and later performance on false belief tasks, tasks which are seen as indices of theory of mind, specifically, or social understanding, more generally. Their approach brings together social and cognitive development research domains, which have proceeded largely in isolation from one another for decades (with several noteworthy exceptions), very like the parable of the learned blind men of Hindustan examining different parts of the social understanding elephant.

To support their thesis, C\&L review research on the impact of social discourse. This research has shown that social understanding develops relatively earlier in children exposed to mental state language in a variety of interactional contexts that include play interactions with peers and siblings, parental discipline, and joint reading with parents (e.g., Meins et al. 2002; Ruffman et al. 2002). Further, a series of training studies (e.g., Appleton \& Reddy 1996; Slaughter \& Gopnik 1996) lends experimental evidence to the claim that exposure to discourse about mental states can enhance children's performance on false belief tasks. Issues arise which include drawing causal inferences from longitudinal and experimental data, the external validity of false belief tasks, and the largely unknown cultural specificity of links between relationship variables, language, and social understanding. But the evidence is compelling.

However, C\&L have been tentative in delineating a mechanism for the developmental relation between interpersonal factors and social understanding. For example, in the concluding comments of this paper, C\&L highlight the recent and persuasive findings of Meins et al. (2002) that mental state discourse of parents predicts children's false belief understanding four years later. They then pose the question: "What is it about the nature of these parents' interactions with their infants that correlates with the development of social understanding?" (target article, sect. 5, para. 4). Reframed, the critical question could be: How does exposure to discourse about mental states lead to enhanced social understanding in children? An answer lies in Vygotsky's mechanism of internalization (see Bruner 1986; Lloyd \& Fernyhough 1999; Vygotsky 1978; 1986).

Vygotsky proposed that children internalize social speech, and such internalization socializes a child's practical intellect. Higherorder thought originates in the internalization of external social relationships and meanings, not by merely imitating the external in the internal, but by recoding what is known about the external into the internal (C\&L's "reconstruction of knowledge," target article, Note 2). Applied to social understanding, mental state discourse leads to young children internalizing the notion that others can have thoughts and emotions that differ from their own. Children experience discourse about thoughts and beliefs of others and integrate such talk into their own behavior. This is fundamental to self-other understanding and passing false belief tasks.

C\&L actually discuss internalization earlier in the article, but it 
is not in Vygotsky's framework. Instead, Piaget and Wittgenstein dominate the theoretical approach of this article, even though $\mathrm{Vy}$ gotsky, and even John Bowlby, are social constructivists who have discussed social mechanisms by which cognitive processes arise. Piaget was simply not a social constructivist. However, emphases on consistencies as opposed to differences between theoretical approaches are critical to developing coherent developmental theory, much like an exchange of information between the blind men from Hindustan before definitively declaring what they have discovered.

C\&L conclude that "researchers studying talk about the psychological world should be concerned not just with mental state terms but more broadly with talk about human activity" (sect. 5 , para. 4). Data suggest that it is discourse about mental states of self and others that predicts social understanding, not discourse about behavioral or physical attributes. Internalization must have a cognitive basis, and this basis may relate back to Piaget's schemas or John Bowlby's working models of self and other. This is the common mechanism of social understanding and relationship processes that is being intensely examined in current developmental research on mental state discourse (see Symons 2004).

Another advantage of Vygotsky's concept of internalization is that variation in language and culture are accommodated as children internalize what they experience in what C\&L call triadic interchanges. Although there are potential variations between groups in language and culture, as well as rate of acquisition of self-other understanding, ultimately all developmentally intact children come to some understanding that others have mental events that can differ from their own: what has been described as a "human universal" in self-other understanding.

But if mental state discourse is socialized into children, is it reflected in children's own language use? Researchers have been careful to examine parents' use of mental state discourse in the presence and absence of children, but have largely ignored children's own discourse about mental states outside of social situations. Children's discourse in solitary tasks may be important to examine. For example, in our recent analyses of children's spontaneous discourse in Canadian and Australian samples (Symons et al., in press), children's use of mental state language during storytelling tasks correlated as high as .62 with performance on a battery of false belief tasks, even with general language ability controlled. It would therefore seem appropriate that children's self-talk about mental states within their sociocultural context be included in any developmental model that leaps from parental mental state discourse to children's social understanding, which C\&L address in their discussion of a Wittgensteinian approach to private language. Internalization can be seen as a social process, but is rendered meaningless unless specific cognitive and linguistic mechanisms are added so that we can address the following: Internalized into what? Encoded how? Carried forward in what fashion? With what meaningful life impact? Answers to these questions cannot be fully addressed from a single perspective.

\section{The mind in the mind of the beholder: Elucidating relational influences on early social understanding}

\author{
Ross A. Thompson ${ }^{\mathrm{a}}$ and H. Abigail Raikes ${ }^{\mathrm{b}}$ \\ aDepartment of Psychology, University of California, Davis, Davis, CA 95616- \\ 8686; ' bepartment of Psychology, University of Nebraska, Lincoln, NB \\ 68588-0308. rathompson@ucdavis.edu araikes1@bigred.unl.edu \\ http://psychology.ucdavis.edu/faculty/pgms/page.cfm?PersonID=156
}

Abstract: Relational experiences shape emergent social understanding, and two influences deserve particular attention. First, parent-child conversation about shared experiences incorporates both implicit and explicit information about mental states that catalyzes the social construction of understanding, especially in juxtaposition with the child's direct experience. Second, emotion infuses the contexts and cognitions about social experiences that provoke the child's constructivist efforts.
There have already been many responses to what Carpendale \& Lewis $(C \& L)$ are calling for in the target article: namely, greater theoretical attention to the influence of social interaction on the development of children's social understanding. These include Rogoff's (1990) constructivist view of the appropriation of social cognition in shared activity, Nelson's (1996) portrayal of the growth of the linguistically mediated mind, and the inquiry of theory-of-mind researchers into social influences on psychological understanding (e.g., Lagattuta \& Wellman 2002). Social developmentalists have also been concerned with the influence of social interaction on mental representation. Attachment theorists, for example, believe that representations (or "internal working models") of people, self, and relationships arise from variations in attachment security and patterns of communication shared within secure or insecure parent-child relationships (Bretherton \& Munholland 1999).

The view that communication within salient relationships shapes early social understanding provides an opportunity to better understand the processes by which social interaction is influential. This is one of the future directions for research identified by C\&L, but we believe there are at least two relational influences that are neglected in their analysis and that deserve greater attention. The first concerns explicit and implicit features of conversational discourse between parents and children. There is now an expanding research literature showing that mothers' conversational style with young offspring - especially, the extent of the elaborative detail, contextual information, and provocative questions mothers provide - contributes not only to the sophistication of children's event representation but also to their understanding of emotions, conscience development, autobiographical memory, and other features of social cognitive growth (Thompson 1998). Maternal conversational references to people's feelings and emotions are also related to young children's emotion and moral understanding (Thompson et al. 2003).

Mothers in secure attachment relationships are more elaborative in conversational discourse (and also make more frequent references to emotion), and this may be one reason for the working models their offspring develop (Thompson 2000). Elaborative discourse about shared experiences in the context of a generally warm, secure relationship may enhance children's receptiveness to the understanding of psychological states embedded within such conversations. For this reason, we are exploring in current research the association between maternal "mind-mindedness" (Meins 1999) and elaborative discourse to elucidate avenues by which attachment security and social cognitive development may be related. This work also offers avenues for clarifying the nature and development of the "internal working models" of interest to attachment researchers (Thompson \& Raikes 2003).

Discourse quality in parent-child conversation is important not only for the reasons identified by C\&L (e.g., as a means for becoming aware of beliefs; as a way of representing false belief in contrast to reality), but also as a means for understanding the social constructivist processes within relationships that they emphasize. In conversation about shared experiences, the secondary representations provided in parental discourse are juxtaposed with the child's direct representations through experience; and the convergence, dissonance, complementarity, and differential focus of these representations are a rich basis for the constructivist processes described by C\&L, because parents and children often perceive shared experiences differently (Levine et al. 1999).

This work therefore significantly expands the authors' proposals for how relationships influence social-cognitive growth in early childhood, and suggests also that relational quality as well as variations in the nature of parent-child discourse - including nonverbal features of parent-child conversations, such as affect, gesture, and context - contribute significantly to representations of mental and psychological functioning of young children. Early parentchild conversations are important not only because they offer guidance to young children concerning the relations between behavior and people's thoughts, feelings, and beliefs, but because 
they also include implicit and explicit moral judgments, intentionality attributions, and even characterological ascriptions to the child that are likely to contribute significantly to the construction of social and psychological understanding.

Furthermore, the context of shared conversation is important. Contrary to C\&L, conflict as well as comity is a salient conversational context because nothing focuses a young child's attention on differing mental states than the realization that conflict with another must be managed. Thus, maternal verbal conflict-resolution strategies in shared conversation during disputes, such as mother's use of justifications, references to emotions, and limited use of threatening or intimidating tactics, predict young children's emotional and moral development months later (Laible \& Thompson 2002).

The second feature of social interaction highlighted by these findings - and largely neglected by C\&L - is emotion. Young children's efforts to manage and comprehend their feelings and the emotions of others are significant catalysts to understanding mental states, and such experiences constitute salient interactional contexts in which mental state differences are the focus of shared observation and communication. This is true from the inauguration of the "epistemic triangle" in infancy, as emotions contribute to self-other differentiation and contexts of shared reference and joint attention (such as in social referencing). Moreover, emotion organizes early understanding of mental states by connecting emotion to broader emergent representations of relationships, self-referent beliefs, and comprehension of behavioral expectations. Emotion is important, therefore, because it is not only thinking about emotion (which has been the focus of much theory-ofmind research) but thinking with emotion about self and others that stimulates early social understanding. This is one reason why in our studies (see Thompson et al. 2003) as well as others (Lagattuta \& Wellman 2002), relational influences are especially apparent in children's conversations about and comprehension of negative emotional events. Constraint and cooperation are both conceptually provocative experiences for young children.

In sum, emotion is often salient to the activity that contributes to social understanding. In early childhood, moreover, conversation about these experiences with an adult who elaborates the child's direct experience, in the context of a warm, secure relationship, provides a rich basis for the child's construction of social understanding. This social constructionist view provides provocative opportunities to integrate the focus on normative development, emphasized by students of early conceptual growth, with the focus on individual differences emphasized by students of early sociopersonality development.

\section{In defense of enculturation}

\section{Penelope G. Vinden \\ Hiatt School of Psychology, Clark University, Worcester, MA 01610-1477. pvinden@clarku.edu \\ http://www.clarku.edu/departments/psychology/faculty/vinden.cfm}

\begin{abstract}
Carpendale \& Lewis (C\&L) view enculturation as the internalization of cultural concepts given in social interactions. They claim that enculturation implies relativism and fails to take into account both the constructive activity of the child and the gradual nature of development. Their view is contrasted with the notion of the child as both enculturated and enculturing throughout the course of development.
\end{abstract}

Carpendale \& Lewis (C\&L) argue against a social construction view, which they see as equivalent to enculturation, or the internalization of cultural concepts given in social interactions. I would claim, however, that enculturation need not be seen as a simply passive process of information transmission (cf. Vinden 2000). Furthermore, if viewed as an interactive process, enculturation is a term that nicely captures the unique role that culture plays in human social development; a role that includes but extends beyond triadic interactions.

The narrowness of C\&L's conception of enculturation is reflected in their discussion of its supposed implications. In the first place, they maintain that enculturation is the importing of "readymade" concepts from the social world to the individual, which implies cultural relativism (cf. target article, sect. 3.2, para. 7). Enculturation need not involve, however, either a unidirectional social transmission model or radical relativism.

At first glance, enculturation may seem to be a one-way street. A newborn child seems little able to engage in even the most rudimentary of dyadic interactions. He or she is fed, moved, changed, spoken to (or not) according to the wishes of the caretaker. Crying may or may not influence these interactions - a responsive caretaker may attune him- or herself to the child, but the child is as yet unable to attune to the other as other. As C\&L point out, young children may not initially understand the social implications of interactions and only gradually move from "behavioristic" interactions (crying brings food) to "behavioristic/mentalistic" interactions (crying makes Mom do what I want) to "mentalistic" interactions (crying makes Mom think I'm hungry). But the child, whether intentionally or not, certainly influences the actions of the caregiver from the moment of birth onwards. A child who comes into the world kicking and screaming, and whose behavior continues, for whatever reason, to be characterized by kicking and screaming in the months and perhaps years to follow, is certainly enculturating her caregiver in a manner quite different from the newborn who is all cooing and contentment.

My suspicion is that there are cycles of directionality in the enculturation process. At some points in the child's life, enculturation may be primarily a learning of the criteria for competent interactions by the novice from the expert. This is what the authors seem to focus on. Though they want to emphasize the active role of the child, they still seem to fall into talk that would imply that much of the learning is of norms (situational/relational norms, not ideational norms) already established "out there" in the world: "children ... learn the appropriate contexts for the use of various words referring to the psychological world" (sect. 3.2, para. 3); they "learn the criteria for words to talk about human activity and then can reflect on the psychological world" (sect. 3.2, para. 9). What C\&L fail to emphasize, however, is that at times learning may be primarily in the direction of child to other, as the other is called upon to adjust to the new abilities or demands of the child by relating in a new way. Enculturation is always by the child of others as well as of the child by others.

Enculturation need not invoke a relativism that leaves one unable to say that some forms of knowledge are "better, more complete, or more adequate" than others (sect 2.1, para. 10). As C\&L clearly point out, there are likely basic universal shared practices that are common to all human experience. But it is also well documented that differences exist among cultures at quite a fundamental level - for example, the degree to which the individual or the group is emphasized (cf. Lillard 1998; Vinden \& Astington 2000 ). If a cultural group puts a low priority on talking about inner states, or does not conceive of them as particularly personal and individual, then the public criteria for learning about thoughts and emotions might be quite different from the typical public criteria in a culture where people are seen as individuals with discrete, personal, private inner lives. Hence, although understanding and getting along with other human beings may be universal, what that means and the criterial situations for learning about getting along can vary widely from culture to culture. And if the criteria vary, why would the understanding constructed from those situations not vary also?

Certain kinds of criterial situations may provide knowledge that is better or more adequate for living in those situations. There is a certain kind of circularity here, but not, I think, a vicious sort. For example, when living on a small atoll in the Pacific Ocean, where one's friends, neighbors, and relatives are all the same people, individuality might not be as adaptive as communality. The 
same might be true for those living on an African-American innercity "atoll" within a predominantly Caucasian middle-class city. Once an emphasis on group rather than individual processes is established, the situations in which that understanding is fostered become criterial and could tend to self-perpetuation. But what may work well on an atoll may not work elsewhere - what is better, more complete, or more adequate in one social or cultural situation may not be so in another.

That being said, there is still no reason per se why cultural variability need go hand-in-hand with a view of passive transmission of mental concepts to the child. The child is influential not only in constructing his or her understanding but also in defining the context in which learning takes place, and even the content of what should be learned. Furthermore, individuals are not the only things that interact in relationship - groups, societies, cultures all relate and influence one another.

In short, although I applaud C\&L's efforts to make social interaction the center of development, rather than merely an add-on explanatory variable, I feel they are not radical enough. Seeing triadic interactions as the epicenter of development does not necessitate relegating cultural variability to the sidelines. Children's development is not constructive only in the sense that they gradually cobble together cultural criteria for language use and other behaviors. It is also constructive because part of their development consists in constructing the criteria for themselves and for others. Without this active transforming of the world, caregivers would never adapt to their children, peers would never learn how to pacify or enrage their playmates, and societal and cultural change would not exist.

\section{Interpretation based on richness of experience: Theory development from a social-constructivist perspective}

\author{
Arlene S. Walker-Andrews and Judith A. Hudson \\ Department of Psychology, Rutgers University, Piscataway, NJ 08854-8020. \\ arlenewa@rci.rutgers.edu jhudson@rci.rutgers.edu \\ http://www.rci.rutgers.edu/ arlenewa/ \\ http://www.rci.rutgers.edu/ jhudson/
}

\begin{abstract}
The view that children's understanding of mind is constructed through social interaction is consistent with other social-constructivist models. We provide examples of similar claims in research on emotion perception, pretense understanding, autobiographical memory, and event knowledge. Identification of common elements from such socio-cultural perspectives may lead to greater theoretical integration and provide a new framework for exploring human development.
\end{abstract}

Carpendale \& Lewis (C\&L) propose that the development of understanding of others' minds grows not from a kernel of knowledge already in a child's brain, nor from the assumption of environmental-cultural norms, but through dynamic, ongoing interactions with caregivers and the physical world. This is a welcome step into the already burgeoning literature in a number of areas - motor development, cognitive development, socio-emotional development - spurred by theories such as dynamic systems, the ecological approach, and contextual approaches. The view that children's understanding of mind is part of the larger process of social understanding and is constructed through social interaction is consistent with other models that attribute a critical role to the process of social interaction in development. We provide examples of similar claims in research on the development of the perception of emotion, pretense understanding, autobiographical memory, and event knowledge. These lines of research provide support for the notion that richness of experience, not rich interpretation, provides an interpretive framework for understanding children's emerging understanding. They also indicate that social interaction is fundamental to human development across a wide range of social, emotional, cognitive, and perceptual phenomena.

C\&L argue that development of an understanding of others' minds (intentions, beliefs, emotions) is not "all or none." Research on infants' understanding of emotional expressions supports this contention. Whether an infant perceives an emotional expression and responds to it depends on the age of the infant, the expression that is being enacted, the task by which the experimenter measures perception, the definition of perception (detection, discrimination, recognition, understanding), and the context in which the expression is encountered (Walker-Andrews 1997). Infants as young as three months show intermodal matching for their mothers' happy and sad facial-vocal expressions, but not for expressions posed by a female stranger (Kahana-Kalman \& Walker-Andrews 2001) Similarly, infants show intermodal matching for fathers' happy and sad facial-vocal expressions only when they have highly involved fathers (Montague \& Walker-Andrews 2002). Lest one think that this is because infants are merely exposed to maternal expressions more than paternal expressions, refer to research by Dunn and colleagues (Dunn et al. 1991a; 1991b) that illustrates the importance of the family context and interactions in the perception of emotion by children.

Children's understanding of the pretense of others also develops gradually (Harris 1994b). For example, Walker-Andrews and Kahana-Kalman (1999) concluded in a study of pretense understanding that "toddlers move from appreciating that an adult partner initiated a game of pretend to understanding the pretend stipulations of the adult, and finally, at 24 months, to using that understanding to enter into collaborative pretend play" (p. 531). In particular, some 15- and 18-month-olds imitated the experimenter's pretend transformations as a way to coordinate actions with the experimenter. To borrow from the description of children's involvement in deception, the toddlers entered into the pretense "with only partial understanding and such experience is the context for learning" (target article, sect. 4.1, para. 6) about such mental states as pretense and deception. The comprehension of another's pretense emerges early in the second year, but the ability to tailor one's pretend actions in that interaction is not demonstrated until later.

C\&L propose that conversation provides a context for children to interpret and talk about the mental world. Conversation also provides a context for thinking and talking about past events, as demonstrated by extensive research on the development of young children's autobiographic memory (Reese 2002). Although children's contributions to conversations at two to three years consist solely of short answers to specific questions, over time, children develop the narrative skills to discuss the past more fully and to eventually construct independent memory narratives (Haden et al. 1997; Hudson 1990; Nelson 1993). Through conversation children learn how to remember, not what to remember; they do not simply repeat what was told to them but acquire memory and narrative skills (Hudson 1990). This distinction is similar to C\&L's argument that through social interaction, children do not simply adopt socially available knowledge, but rather, construct their own understandings within the interactional context. Research has also shown that individual differences in parents' reminiscing style affect children's independent narrative ability in later years, indicating that characteristics of the dyadic relationship influence children's autobiographic memory development (Reese et al. 1993).

Finally, research on the development of event knowledge illustrates the tension between performance factors and underlying competence discussed by C\&L. A large body of research has shown that children's generalized event representations (GERs) provide a cognitive context for the development of memory, planning, narrative, inferential reasoning, and temporal understanding (Nelson 1986). Before children display these skills in novel, decontextualized, experimental tasks, they accurately use temporal language, draw appropriate inferences, construct future plans, remember stories and events, and construct story narratives when reasoning about familiar events in meaningful interactive contexts 
(Hudson 1993; Hudson et al. 1995). This reprises the issue of the role of partial understanding in development. Researchers examining the role of event knowledge in children's cognitive development have argued that knowledge displayed within familiar contexts provides the foundation for later, more generalized skill. Just as a lexicon for talking about the mental world provides children with a cognitive resource for reflection and interpretation, children’s GERs allow initial "understanding-in-action” to be consolidated through reflection on internal event representations.

We propose that a more social-interactional approach is needed at this stage of social-constructivist theory development. All of these lines of research stem from approaches emphasizing the social-cultural embeddedness of development. Although specific mechanisms may vary, they constitute a "family" of social constructivist approaches. Our single concern with the C\&L model is that it may not be adequately inclusive. The tendency to label as "passive enculturation" approaches that vary in small degrees from the C\&L position may limit the potential for integration across research domains. Rather than focusing on differences from an individualist framework, joint attention on common principles and collaborative interactions may be more fruitful for theory development.

\section{What infants know about intentional action and how they might come to know it}

\author{
Camille Wilson-Brune and Amanda L. Woodward \\ Department of Psychology, University of Chicago, Chicago, IL 60637. \\ camillew@uchicago.edurwoodward@uchicago.edu \\ http://www.ccp.uchicago.edu/faculty/Amanda_Woodward/
}

\begin{abstract}
Carpendale \& Lewis $(\mathrm{C} \& \mathrm{~L})$ propose that social knowledge is constructed from triadic interactions. This account generates testable predictions concerning social knowledge in infancy. Current evidence is not entirely consistent with these predictions. Infants possess action knowledge before they engage in triadic interactions, and triadic use of an action does not always precede knowledge about the action.
\end{abstract}

Carpendale \& Lewis (C\&L) propose an ontogenetic relation between interacting and knowing: By participating in increasingly well-organized social exchanges, children come to construct a theory of mind. Evaluating this proposal requires measuring both social actions and social knowledge. Given this requirement, C\&L's review reveals striking gaps in the empirical record. The infant work presented concerns assessments of social behavior, although underlying social cognition (or lack thereof) is only inferred. The studies of older children concern social cognition as assessed in interview studies, with factors such as parenting style standing as proxies for children's social interactions. A full account must address both gaps. We will focus on the first.

C\&L propose that triadic interactions, in which the infant and caretaker mutually coordinate attention on the same object, are necessary for the construction of social knowledge and, ultimately, a theory of mind. Because infants do not engage in triadic interactions until the end of the first year of life, this account predicts that they have not yet begun to construct social knowledge. C\&L further predict that, once triadic interactions are established, social behavior relevant to a particular aspect of intentional knowledge will emerge before the knowledge itself does. Recent evidence from our laboratory and others' speaks to these predictions. This evidence indicates that triadic interactions and social knowledge do not always travel together in ontogeny.

Aspects of social knowledge are present months before infants engage in triadic interactions. Infants represent actions not as purely physical motions through space but rather as directed at objects or states of affairs (Baldwin et al. 2001; Csibra et al. 2003; Gergely et al. 1995; Moore 1999; Woodward 1998; Phillips et al. 2002). To illustrate, in one study (Woodward 1998), 6-month-old infants viewed a person reaching for and grasping an object. Following habituation, infants demonstrated a stronger novelty response to test events that disrupted the relation between agent and object than to test events that maintained this relation while varying the spatial properties of the reach. Infants did not respond in this way when viewing inanimate objects that touched or grasped other objects, or when viewing manual contact that appeared purposeless to adults (Woodward 1999). Therefore, infants' social knowledge reflects a foundational aspect of mature conceptions of intentional action - namely, that certain human actions are object-directed (see Barresi \& Moore 1996).

This work highlights infants' knowledge about instrumental actions. C\&L focus on interactions in which infant and caregiver share attention, as expressed by looking and pointing. But these are just a subset of the actions that adults view as intentional. Indeed, many investigators have elucidated infants' developing ability to extract the goals behind observed instrumental actions (Gergely et al. 2002; Meltzoff 1995; Wenner \& Bauer 1999; Woodward \& Sommerville 2000). Our recent findings are consistent with the thesis that experience contributes to infants' construction of social knowledge; however, in this case what matters appears to be infants' experience of acting on objects rather than of participating in triadic interactions (Sommerville \& Woodward, in press).

Recent studies also elucidate infants' knowledge about the actions involved in triadic exchanges. Infants begin to follow gaze during the first year of life, but, as many have noted, this observation alone does not tell us whether infants understand the "looking at" relation (e.g., Barresi \& Moore 1996). Several studies indicate that by 12 months, infants encode looking and pointing as relational (Moore 1999; Phillips et al. 2002; Woodward 2003; Woodward \& Guajardo 2002). Prior to 12 months, infants respond to gaze by orienting their own attention but seem not to encode the relation between looker and object (Woodward 2003). This pattern of findings is consistent with C\&L's proposal. Infants begin to understand the looking relation after several months of responding appropriately to shifts in others' gaze.

However, the emergence of pointing suggests that this pattern does not hold in all cases. Knowledge about pointing is evident before infants employ it robustly in triadic interactions. Infants' first points are often described as indexing their own attention rather than being communicative (Bates et al. 1979; Schaffer 1984a). It is not until 12 to 15 months of age that infants produce points in a clearly communicative manner and follow others' points to their distant referents (Bakeman \& Adamson 1986; Carpenter et al. 1998; Desrochers et al. 1995). Furthermore, infants do not use contextual cues to determine when to point until around their second birthday (e.g., Dunham et al. 2000; Moore \& D'Entremont 2001). Consistent with the thesis that experience is related to knowledge, at 10 months, those infants who produce object-directed (but not clearly communicative) points understand observed points as relational (Woodward \& Guajardo 2002). Therefore, although experience may contribute to infants' knowledge about pointing, the evidence suggests that the relevant experience is not triadic in nature.

To conclude, recent findings indicate that although triadic interactions may be one source of infants' social knowledge, they are not the sole source. Infants are sensitive to the object-directedness of instrumental actions well before the onset of triadic interactions and come to understand certain actions before using them in triadic interaction. In addition to interaction, firsthand agentive experience is a likely contributor to this system of knowledge. That is, there seems to be more than one route into social understanding. This may account for the fact that all normally developing children construct a theory of mind despite the existence of broad cross-cultural variation in the nature of early triadic interactions and habits of talk about the mind (Lillard 1998; Rogoff et al. 1993). 
Response/Carpendale \& Lewis: Constructing an understanding of mind

\section{Constructing an understanding of mind with peers}

\author{
Stephanie Zerwas, Geetha Balaraman, and Celia Brownel \\ Department of Psychology, University of Pittsburgh, Pittsburgh, PA 15260. \\ stz1@pitt.eduｇrb22@pitt.edu brownell@pitt.edu
}

Abstract: Carpendale \& Lewis (C\&L) stress the importance of social interaction for social understanding, but focus on the adult-child relationship. In the present commentary, we discuss the development of social understanding within early peer relationships. We argue that peer interaction stretches the limits of early social understanding, thereby providing both unique challenges and unique opportunities for constructing an understanding of others' minds.

In their target article, Carpendale \& Lewis (C\&L) assert that children construct social understanding through interactions with others and that social knowledge is fundamentally based on action within these relationships. In particular, they emphasize that early triadic interaction facilitates the development of language and that conversation about the mental world, in turn, permits children to perceive, understand, and reflect on their own and others' mental and emotional states. We share the authors' view that social interaction contributes to social understanding. However, we believe that the authors have limited their argument by focusing so heavily on early parent-child interaction. In the present commentary we discuss the contributions of early peer interaction to social skill and social understanding. Although C\&L acknowledge the "sibling effect" (Perner et al. 1994) and the role of cooperative interactions with age-mates for children's use of mental state terms (Brown et al. 1996), they do not discuss how interactions with siblings and peers differ from adult-child interactions, and the possibly unique role played by interactions with other children in generating early social understanding.

C\&L argue that "children's initial, fragile social understanding" is "at first evident when supported by social interaction" (sect. 4.1, para. 9, emphasis ours) and that "children gradually construct social understanding through the regularities they experience in interacting with others" (sect. 3, para. 1, emphasis ours). In their formulation, the young child's adult partner supports social engagement through regular and routinized interaction and, in that context, interprets and labels the child's mental states. From our view, this type of cooperative interaction, although probably initially necessary for children's nascent social understanding, can take children only so far toward a more general understanding of mind. In routinized interactions, especially with a sensitive adult who has complex mental state understanding, the child has little need to predict the partner's behavior or to communicate his own mental states. When social partners' behavior is predictable, and when routines and communication are either built-in or highly learned, having to read minds is not necessary.

Peer interaction, in contrast, provides a social context in which children's partners are unpredictable and their goals, intentions, and desires are often difficult to read even for sensitive, mindminded mothers. The challenges of interacting with peers may require that children be explicit about communicating their intentions. Consistent with this notion, Smiley (2001) found that toddlers were more likely to state their future intentions in peer play than in mother-child interactions. Furthermore, peer interaction also provides children their only opportunity for true cooperation, defined by both Piaget (1932/1965b) and C\&L as "interaction among equals." Thus, peers pose both unique challenges and unique opportunities for the growth of early social understanding.

Children are capable of establishing and maintaining positive peer interactions in play and in cooperative problem-solving by the end of their second year (Brownell et al. 2003; Eckerman et al. 1989; Howes et al. 1989). Without a way to "make sense" of other children's behavior, the toddler cannot anticipate a peer's behavior, hence cannot behave in a coordinated, reciprocal, ac- commodating manner, and peers' behavior will simply be inscrutable for the very young child. The fact that children can imitate one another by 14 to 18 months (Eckerman et al. 1989; Hanna \& Meltzoff 1993) but cannot cooperate with one another until 24 months or later (Brownell \& Carriger 1990; Brownell et al. 2003; Tomasello et al. 1993) suggests that their early understanding of others' minds, as constructed in adult-child relationships, remains too incomplete to serve interaction more generally. Moreover, the social understanding that develops in the context of early peer play is not a function of mental state talk between peers. Nonverbal interaction may be even more important for children's developing social understanding of one another. Mutual imitation, for example, may provide the first means for children to begin making sense of their peers' behavior and may be the grist for generating initial understanding of peers' intentions (Eckerman et al. 1989).

Social understanding not only arises in situations of peer cooperation and positive interaction around a shared theme, goal, or desire, but may also be adaptive and necessary in instances of competition. It is notable that possession struggles and conflicts, which constitute a relatively high proportion of early peer interactions (Brownell \& Brown 1992; Hay \& Ross 1982), are unique to childchild interactions. When one toddler approaches another, the child must figure out whether the peer intends to hug her, play with her, take away her favorite toy, or something else. Caregivers are not always available to assist children in interpreting and understanding such situations, and even when they are present, they often prefer to let children figure it out on their own. Hence, children may be motivated to learn how to read other children's mental states and to understand the relations between mental states and behavior so that they can effectively negotiate ambiguous, emotionally charged situations. Such interactions may also provide children insight into the nature of their own emotional experiences (Balaraman et al. 2003).

We do not mean to suggest that parent-child relationships and peer relationships are unconnected, however. Young toddlers who are more responsive to an adult's bids for joint attention are also better at coordinating their actions with a peer toward a common goal (Brownell et al. 2003). Toddlers who have more positive experiences in play with their mothers engage in more complex social play with their peers (Howes \& Stewart 1987; Zerwas \& Brownell 2003). Thus, the social understanding children construct during interaction with adults is also brought to bear on their interactions with peers. Nevertheless, establishing and maintaining positive interactions with peer partners pose distinctive challenges both affectively and cognitively. Peer interaction thereby stretches the limits of children's early social understanding and makes unique contributions to its development.

\section{Authors' Response}

\section{Constructing understanding, with feeling}

\section{Jeremy I. M. Carpendale ${ }^{\mathrm{a}}$ and Charlie Lewis ${ }^{\mathrm{b}}$ \\ a Department of Psychology, Simon Fraser University, Burnaby, BC Canada V5A 1S6; 'bepartment of Psychology, Lancaster University, Lancaster, LA1 4YF, United Kingdom. jcarpend@sfu.ca c.lewis@lancaster.ac.uk http://www.psych.lancs.ac.uk/people/CharlieLewis.html}

Abstract: We explore three types of criticisms of our theory on the development of children's social understanding. We reject suggestions that we offer nothing new to traditional theories of development or recent "social" accounts of "theory of mind." Second, we take the point that there are grounds for improving our 
Response/Carpendale \& Lewis: Constructing an understanding of mind

account of dyadic interaction in infancy but reject claims that we have not sufficiently accounted for how we incorporate the notions of criteria and structure into the theory. Third, we accept that the epistemic triangle, as defined, would benefit from an affective dimension and such a formulation could be used to describe the dynamic of developmental change from infancy to beyond early childhood. We still feel that the combination of Wittgenstein, Vygotsky, and Piaget remains as an antidote to the flaws in current "theories of mind" approaches to social understanding.

The squirrel does not infer by induction that it is going to need stores next winter as well.

—Wittgenstein, On Certainty (1969, para. 287)

For us the starting issue was that the dominant theories in the area of the development of children's social understanding, referred to as "children's theories of mind," view the problem that children must solve as inferring the presence of and learning about unobservable inner mental states. Wittgenstein's rejection of the idea of "squirrel cognition" summarizes our suspicions about an account of early human development that imputes the child with a social understanding that develops in an individualistic manner not grounded in shared practices. The dominant accounts initially made little reference to the child's experiences except to examine the young theoretician's database, although they are now accommodating to research on children's social experience. Hence, we turned to Wittgenstein and the very traditions within developmental psychology that the theory of mind tradition set itself up against. The target article was an attempt to pull together these diverse views in order to get a child's eye view of development. This we assume to be much more piecemeal and embedded in experience than recent accounts have suggested.

We have been both moved and inspired by the commentaries and strongly recommend them as worthwhile reading. In writing this Response, we feel we could have done more justice to about half the number and apologize to those who we simply mention; but we were restricted to about 200 words per commentary. So we have concentrated on some much more than others - and mainly those we felt challenged us more (they tended to be those we thought were closest to us in theoretical terms). The response is divided into three sections: general criticisms, specific concerns with our theory, and the many elegant extensions that were recommended. Far from criticizing our plea to make theories of the development of social understanding more socially embedded, many of these commentaries emphasize just how rich the child's social experiences are and how we did not go far enough.

\section{R1. Is our theory needed? Or should it be upended?}

There are three ways in which the commentators question whether our position is necessary: One group, who are discussed in the first subsection that follows, takes the long view and suggests that our approach is hardly new in its distillation of fine theories. The second part deals with a different view - that we have been trumped by recent socially embedded criticisms of theory of mind - and we discuss this in section R1.2. Finally, in sections R1.3 and R1.4 we reflect on the view that traditional accounts of theory of mind worked just nicely without the critical analysis that we offer, particularly the need for a Wittgensteinian analysis.

\section{R1.1. Piaget and Vygotsky - again?}

We are pleased that many of our commentators agree that a social perspective has long been neglected in the area known as children's theories of mind. And, like them, we believe it is needed. But have we simply recycled classic developmental theories in this article? Certainly, Lourenço assumed that we were making more grandiose claims about proposing a completely new theory than we were. ${ }^{1}$ We make it clear that we draw on Piagetian and Vygotskyian theory - ideas with extensive histories. Thus, we agree with Chesnokova that the ideas we are working with have long histories, and we thank her for adding Luria's name to the list of theorists we rely on. Sokol \& Lalonde also bring in Dewey and this list could be extended considerably to include the influence of Janet, Baldwin, Pierce, Werner, Bühler, and G. H. Mead. Tracing the extensive histories of the ideas in question was beyond the scope of our target article and we refer readers to excellent sources such as Overton (1998a) and Valsiner (1998).

So, is it "new" to draw upon Piaget and Vygotsky? Lourenço asserts that "without additional specifications and elaborations" Piaget and Vygotsky's theories "do not need to be repeated." Ironically, he has felt it necessary to contribute to the task of repeating these ideas to fend off common misinterpretations embedded in the scientific folklore (Lourenço \& Machado 1996). In our defense, there is nothing we know of in Piaget and Vygotsky's work about issues equivalent to Wittgenstein's notion of criteria. This we draw from Chapman's (1987a; Carpendale et al. 1996) work on operational thinking and extend to children's reasoning about social matters. As many commentators point out, this is no straightforward matter and we turn to some of these issues below.

Perhaps implicitly criticizing our reliance on Piaget, Symons and Fernyhough suggest that we should instead rely more on Vygotsky. Vygotskian ideas, as Chesnokova rightly points out, are embedded in our approach. ${ }^{2}$ Symons argues that we are tentative in outlining a mechanism of development and suggests we should employ Vygotsky's idea of internalization. However, this mechanism is far from fully specified, and Vygotsky himself acknowledged that "as yet the barest outline of this process is known" (1978, p. 57). Clearly, Vygotsky's theory needs to be fleshed out, as Chapman (1991) and Fernyhough (see also Fernyhough 1996) have done to some extent. Thus, we maintain that there is no necessary incompatibility between Piaget's views and Vygotsky's views on social relations and we feel that the former's account of internalization is required to flesh out the ideas of the latter.

\section{R1.2. Are we merely repeating current social constructivist theories?}

A few commentators imply that our ideas echo recent ones within the theory of mind tradition (see explicit statements in Astington and Steele). Astington questions how new our approach is and groups us with a family of similar theorists, some of whom are also commentators (Astington, Fernyhough, Montgomery, Nelson; see also Harris, in press). Astington is correct in pointing out areas of agreement between our article and the theorists she lists. We could also go further than Astington in identifying theorists with whom we have much in common concerning infant development, such as Hobson, and Barresi \& Moore. We 
Response/Carpendale \& Lewis: Constructing an understanding of mind

cite most of these authors in the text and acknowledge how they have influenced our ideas. However, we suggest both that there are clear differences between the authors she groups together and that we differ from all of them. For some, the differences relate to matters of detail. For example, Montgomery and Fernyhough still need an account of social development in infancy in order, among other reasons, to overcome the problems Bickhard points out in his commentary concerning the development of prelinguistic normativity. We agree with Astington and Howe that Judy Dunn's work has been seminal in establishing a need for a socially embedded model of mental state understanding and our theory is premised upon that assumption. It is work like Dunn's that stimulates a need for a detailed theoretical account and we suggest that a constructivist one provides an answer.

Many of the theorists cited by Astington differ from us over how children learn the meaning of mental verbs. For example, she groups us with Garfield et al. (2001). Here, if we stick to the general issue of the importance of language and social interaction, we could say we are in agreement. Indeed, Garfield et al. coordinated a great deal of evidence and advanced convincing arguments. However, we differ on the very starting problem that children have to solve. That is, are mental states some sort of hidden inner object that must be inferred? Like Montgomery but unlike others in Astington's list, we suggest that this is the wrong way of setting up the problem that children must solve. Bickhard and Sokol \& Lalonde also present complementary positions in their commentaries. Astington acknowledges that there are differences, but considers these to be only details. However, this is where the devil is, and discussion of such differences is essential for further theoretical development.

There is an interesting implication in the fact that Lourenço and Chesnokova say the theory is old and they mean it is Piagetian and Vygotskian, whereas Astington says it is old but she means it is the same as a family of theories including Nelson (1996), Harris (in press), and herself. But someone has to be wrong, or not quite right. Unless we are mistaken, the theorists listed by Astington either do not mention Piaget or explicitly distance themselves from Piaget's fundamental ideas (see, e.g., Perner \& Astington 1992). It seems that everyone recognizes something of themselves in our approach. Alternatively, it could be that they might be happy with the interpretation of Piaget that we draw on.

Lourenço further argues that our proposal is vague and ad hoc, and that a weakness of our approach is that we can explain anything by it. It is clear from the commentaries that this is a weakness shared by all the other theories in this area. German \& Leslie argue that modular theory has no difficulty accommodating to the evidence that social interaction is important in social development. In fact, they now allow the inclusion of domain general processes in their model, which indicates that they are moving away from most other modular positions, apparently towards theory theory. Gerrans (2002, p. 308, original emphasis) has suggested that "on any view of modularity, the operation of a module cannot be affected by central, domain general processes." Similarly, several commentators (Bartsch \& Estes, Montgomery and Ruffman) argue that theory-theory can also deal with the evidence. The commentaries arguing that the modular and theory theory accounts explain the data that we present only discuss the data on social relationships, notably sibling interactions. We concede that these perspectives can be accommodated to such evidence, but, as noted in our target article (sect. 2.2), as the range of data widens this task becomes more difficult and their theories become more diffuse. Indeed it is getting quite hard to distinguish between these two positions:

"theory of mind" is described as having an innate and possibly modular basis, not as consisting in its entirety as either innate or as a module. (German \& Leslie, original emphasis)

It is certainly true that there are some innately given kinds of psychological knowledge. However, it seems to us that these are most likely to be "starting state" theories. (Gopnik \& Wellman 1992, p. 168)

Lourenço also claims that we have not conclusively ruled out the other theories in this area. First, even if we had devoted our target article to criticizing current theories (and more criticism is provided by commentators Hobson, Mitchell, and Montgomery), we still could not conclusively rule them out. They are not single theories, but rather families of theories sharing core assumptions or research programs in Lakatos's (1970) sense. Research programs cannot be simply ruled out because a modified theory based on the same core assumptions can always be proposed. Second, our criticism based on Wittgenstein is more far-reaching because it reveals flaws in an assumption on which all the dominant theories are based (Chapman 1987a; Montgomery 1997; 2002). The aim of the next subsection is to show that such an approach is a firm conceptual foundation on which to build (see sect. 2.2 in our target article).

\section{R1.3. Is theory theory an adequate explanation? Or do we need Wittgenstein?}

Ruffman's final paragraph presents some interesting attempts to reconcile the theory and constructivist perspectives, but does not bridge a fundamental difference between our approach and the theory theory in how children learn the meaning of mental state terms. Montgomery makes the point that the sibling data can be used to support the positions we criticize, but then goes on to elaborate on the argument from Wittgenstein. He writes that according to the theory theory (and, we would add, simulation and modular theories) children learn the meaning of mental state terms by means of a referential association with private inner sensations that are causally related to but distinct from outward behavior. Section 3.2 of the target article presents arguments like Montgomery's current and recent work (see Montgomery 1997; 2002). Bickhard's discussion of the frame problem adds to the argument that beliefs are not objects that can be learned about through introspection. Bickhard points out that in the case of talk about beliefs there "is nothing unitary, no coherent kind of matter of fact, for belief talk to refer to." The frame problem shows that there is no limit to the number of beliefs a person could be said to have.

Sokol \& Lalonde point out that Dewey's ideas about mental states are consistent with Wittgenstein's later work. One misunderstanding that might be possible after reading their commentary that we want to guard against is the claim that we are denying the existence of mental processes. It might appear that Wittgenstein denies mental states, and therefore he must be a behaviorist. If Wittgenstein denies that a sensation is a mental object, it may appear that he is 
saying there is no sensation, in which case the child's understanding of mind amounts to no more than learning to talk about mental states through enculturation. But that is not what he intended: "And now it looks as if we had denied mental processes. And naturally we don't want to deny them" (Wittgenstein 1968, para. 308). "To deny the mental process would mean to deny the remembering; to deny that anyone ever remembers anything" (Wittgenstein 1953/ 1968, para. 306). What Wittgenstein did reject was labeling mental processes as private objects in the same way we label physical objects. Thus, his argument is about how our language makes us think about psychological processes, not a denial of psychological processes.

Our use of Wittgensteinian ideas is questioned by Shotter, although we note that he does not challenge our philosophical interpretation. Shotter argues that Wittgenstein should not be taken piecemeal and that he was concerned with description not theory. To deal with this criticism we need to apply Wittgenstein's own method to be clear about both the task he took on and the sense in which he used the term "theory." In the paragraph cited by Shotter, Wittgenstein (1953/1968, para. 109) goes on to explain why his considerations could not be scientific ones. It is because the problems he addresses are not empirical but philosophical, focusing exclusively on the meaning of words, due to us being misled by the workings of our language: "Philosophy is a battle against the bewitchment of our intelligence by means of language" (para. 109). His analysis leads to the conclusion that words are torn free from their natural context, and meanings of words only exist in particular contexts. It is this crucial insight that we draw upon in our analysis. Using this idea is not incompatible with the study of human development. Indeed, there are two reasons why we feel justified in using it in our developmental account. First, we should begin from the foundation that Wittgenstein has cleared. In Wittgenstein's words: "I am not interested in constructing a building, so much as in having a perspicuous view of the foundations of possible buildings" (Wittgenstein 1980 , p. 7e). This does not preclude what is built on these foundations. Indeed, secondly, after he disposes of our common misconceptions about how children learn the meaning of words for inner sensations, Wittgenstein goes on to answer his own question of how children learn the meaning of such words by writing: "Here is one possibility ..." (1953/1968, para. 244). It is just this possibility that we are exploring in our theory. This runs counter to Shotter's relativistic stance on Wittgenstein, but we are agreed that these ideas provide a damning critique of the dominant theories of theories of mind.

We should consider why Wittgenstein felt that theory was not appropriate for the task he took on. Given his concern that words only have meaning in particular contexts, a general set of rules for mechanistically deriving meaning (i.e., a theory in one sense of the term) cannot be formulated, because meaning is not mechanistic (see Goldberg 1991). Instead, meaning is indexical. It depends on the location of words or utterances in sequences of interaction (Budwig; see also Suchman 1987; Turnbull 2003). This issue can be illuminated by considering a parallel debate between Searle (1992) and Schegloff (1992) on whether a theory of conversation is possible. Searle concluded that a theory of conversation is not possible. In one sense of the term "theory" Searle (1992) is correct. That is, it is not possible to form a theory that would allow us to derive mean- ings of utterances in conversation because such meanings always depend upon their location in particular sequences of interaction. However, this does not stop us from learning a great deal about the process of conveying meaning in conversation, as shown by Schegloff (1992). The indexical nature of meaning pointed out by Budwig is part of a general problem of the development of knowledge that leads us to a constructivist view, including how children learn the meanings of mental state terms.

\section{R1.4. Is modular theory an adequate explanation? Reductionism and constructivism revisited}

German \& Leslie object to our presentation of the modular view, and they especially object to our contrast between this and the position that humans have evolved the capacity to develop an understanding of mind. Instead, they insist that it is the latter position that they endorse as the modular position. In fact, the statement that humans have evolved the capacity to develop social understanding is just a logical necessity. Within that general agreement there is obviously a lot of controversy. In support of their case, German \& Leslie cite a study by Hughes and Cutting (1999) reporting a strong heritable component $(60 \%)$ to false belief performance. However, in their more recent research with a sample 10 times the size, Hughes et al. (in press) found a much weaker relation: only $16 \%$ genetic influences compared with $63 \%$ environmental influences (see also Deneault et al. 2003). Furthermore, such findings tell us nothing about the process through which any genetic influences might work.

German \& Leslie quote Roth and Leslie (1998), who state that the theory of mind mechanism is essential because it "allows the young brain to attend to ... mental states despite the fact that such states cannot be seen, heard, felt, or otherwise sensed" (emphasis in original). They go on to state that what is critical is the ability to attend to mental state properties such as pretending and believing. However, what it could possibly mean for the child to attend to believing is far from clear in their account. The Wittgensteinian argument above and in section 3.2 of our target article shows that they have gone wrong from the very start because the question they address is misconceived - mental states cannot simply be "attended to" (see also Bickhard, Montgomery, Sokol \& Lalonde). The fact that German \& Leslie do not recognize our position as a proposal implies that their starting assumptions differ so much from ours that they have a different notion of just what a proposal should look like. Yet we call their starting assumptions into question.

Thus, there is an even more fundamental problem with German \& Leslie's account that is shared by two other commentaries. Their approach is reductionistic in their talk about how the "young brain attends" to mental states, and this reduces attending to neural activity (Brown 2003). Craig \& Barrett take a stronger position than German \& Leslie in assuming that folk psychology is innate, due to "certain devices inside us" (Millikan 1993, p. 63). Furthermore, they claim that it is a job for neuroscientists to look for those devices. Gerrans takes a similar tack, treating Wittgenstein's investigations as irrelevant for development psychologists who, he states, should really be concerned with discovering the "neurocognitive processes" involved in concept formation. In our view, a Wittgensteinian analysis undermines the leap that these commentators make so ef- 
Response/Carpendale \& Lewis: Constructing an understanding of mind

fortlessly between neural activity and psychological constructs.

Neurological research will not address the questions we are concerned with because it does not deal with meaning. Craig \& Barrett exemplify the problems inherent in a reductionist approach in their claim that the only thing they are certain of is that knowledge of the world is embodied in one's particular configuration of synaptic connections. Why stop here? Wouldn't it be even more certain to go to the level of the distribution of neurotransmitters at the synapse or at an even finer biochemical level? Synaptic connections may contain information, in some sense of the word, but not knowledge. A train schedule has information but no knowledge; a person is required to read and interpret the schedule. Therefore, a homunculus is required (Heil 1981; Kenny 1991; Müller et al. 1998a; 1998b). In addition to focusing on neurocognitive mechanisms, Gerrans also takes a computational approach. This is fundamentally flawed because there is no way to deal with meaning. "Computers don't understand anything, nor do they care" (Hobson 2002 , p. xiv). The problem for this sort of approach is to "explain the transition from causal processes in the brain to conscious, meaningful experience" (Müller et al. 1998a, pp. 229-30. For similar arguments, see Hacker 1991).

It is for this reason that we are committed to the alternative, constructive approach to development. Siegal asks whether the constructive view can account for the development of specific beliefs, distinguishing these from "core cognitive architecture." Exactly the same process is involved. From a constructive view of knowledge development, concepts cannot simply be transmitted ready-made. Rather, understanding of all types must be constructed by the child in interaction. Nevertheless, our constructive approach is consistent with evolutionary theory. We take the point from Eddy, Fonagy, and German \& Leslie that it is important to consider what biological adaptations allow humans to develop social interaction and social understanding. These make language, human history, and culture possible (Elias 1978). In addressing this problem, we follow Tomasello (1999b) in being particularly cautious about attributing innate knowledge to infants. The problem is not simply solved by saying that infants are born being able to attend to mental states. It is still necessary to explain how an ability evolved. Furthermore, if it is possible for it to have evolved we need an explanation for why it could not develop in individual ontogeny (Bickhard 2004).

Eddy considers a possible interpretation of Povinelli and Eddy's (1996) findings that although chimpanzees follow gaze they do not demonstrate an understanding of the psychological implications of seeing. He notes the possibility that the chimpanzees may have failed their task because they were responding to humans and not to other chimpanzees. Although we agree with Eddy that it is an unlikely explanation, the possibility remains to be evaluated. However, it is evident that we humans do not restrict mentalistic interpretations to our own species, because we had assumed that when chimpanzees follow gaze they also understand the psychological implications of seeing just as we do. If it were found that chimpanzees did restrict their social understanding to members of their own species it would indicate a difference in their social understanding compared to that of humans. In any case, there is additional evidence that a range of increasingly complex forms of gaze following can be observed in human infants.

\section{R2. Should the theory be amended?}

Although some of the commentators discussed in section $\mathrm{R} 1$ are keen to maintain that traditional theoretical positions remain intact in the face of the evidence we summarize, a second group examined the internal consistency of our theory, and we address these here. In the three parts of this section we consider claims from a number of angles that the notion of the epistemic triangle does not fit very well in describing infants' social skills (R2.1), that Wittgenstein's notion of criteria exposes us to a diversity of possible problems (R2.2), and that we have not specified the nature of the structures or processes that the child develops (R2.3). All these analyses call for amendments to the original thesis.

\section{R2.1. The development of the epistemic-relational triangle in infancy: Getting dyadic}

Do we give a full enough explanation of infant development? For a start, as Bickhard argues, Wittgenstein did not account for prelanguage normativity. We agree. Wittgenstein said that the use of words is built onto previous shared practices but he did not explain how these practices arise except to say that the child is "trained." Such practices are where Wittgenstein said he hit bedrock and his spade was turned. But this is where Piaget kept on digging (Müller 1999). And for this part of our argument we rely on Piagetian theory in accounting for development in infancy.

It is Chapman's (1991) notion of the epistemic triangle that helps us build a social dimension in a Piagetian approach to infancy. Does this formulation stand up to the critical scrutiny of our commentators? Ruffman is concerned because he suggests that there is evidence of an early developing self-other distinction. He cites two examples. One is at 18 months of age when we too would obviously expect a self-other distinction to have developed. He also refers to evidence from research with infants from 3 to 4 months of age. The study cited by Ruffman (Rochat \& Striano 2002) was with 4- to 5- and 8- to 9-month-old infants. There was evidence of longer looking time in the other condition compared to the self condition, as well as development between the two age groups. However, Rochat and Striano acknowledged that there were a number of differences between the conditions in addition to self versus other that make firm conclusions difficult. And infants' ability to respond differentially tells us little about whether they have any understanding of a self-other distinction (Müller \& Carpendale, 2004). However, in a developmental account such as ours, early forms of self-other knowledge must be looked for. Therefore, we agree with Rochat and Striano $(2002$, p. 44) that these abilities emerging at 4 to 5 months form the "foundation from which children can eventually develop the conceptual and explicit sense of themselves expressed by the middle of the second year."

The problems to be solved in infant social development are set up very well and in different ways by Barresi \& Moore and Hobson. Our position is very similar to that of Barresi \& Moore. We agree that social understanding develops out of engagement with others - that it is "always out of shared activity with others that advances in understanding of mental phenomena are first made." We recognize 
that two individuals have independent perspectives of the object and of each other; but there remains one issue of possible disagreement between us and this concerns the nature and development of representation. Barresi \& Moore’s use of terms like "reflective representation" and "integrating information" may buy into a theory of representation which is at odds with the activity-based view that we hold (Müller et al. 1998b). However, as we discuss below, their comments on emotional relations and the "we"-ness of interaction are well-taken.

Hobson holds that theories of infant social development must account for primitive modes of experiencing of self and other in interpersonal relations. One of his questions concerns how infants are aware of persons as having a mental as well as a physical dimension. This remark could be misunderstood because Hobson is usually at pains to deny that the initial task for the infant is to discern mental states (Hobson 1994). Rather, he is usually interested in accounting for the formation of the self-other distinction and, like us, he locates this in the dynamic of interaction between the infant and the other. We are also cautious about the solution Hobson proposes. He suggests that "humans are equipped with a propensity for forms of role-taking that both link an individual infant or child or adult with someone else, and at the same time register the distinctiveness of self and other." It is important to know exactly what is meant here because the term "role-taking" would usually be interpreted at a much higher level (Flavell et al. 1968). We assumed that Hobson (and he has confirmed this via email) does not intend it in this way and his commentary goes on to describe this process as: "children are moved by the attitudes of others." Perhaps all that is really needed is a propensity to engage with other people (helped by infants' predisposition to be interested in others' eyes and faces, as Ruffman suggests) and the ability to respond to their reactions, initially without any clear idea of the other as separate from the self.

Hobson is critical of our focus on action and instead suggests that what is needed in addition is an emphasis on emotional engagement. We agree with the addition. Furthermore, he asks us how communication is grounded. From our perspective it is embedded in interaction. This starts with a pattern in which the infant emits affective signals that are punctuated in protoconversation by the adult (see, e.g., Kaye 1982; Schaffer 1977). Gradually the infant's contribution to the interaction becomes more active, in terms of responding to others on this affective level. At first this is dyadic, without reference to objects (Chapman 1991). The goal is just the reaction, there is no communication outside that. Research over the past thirty years has suggested that infants treat humans differently from objects because they react contingently (Watson 1972), and caregivers are intent on extending the length of bouts of affective engagement with very young infants (Stern 1977). We contend that a much leaner interpretation can be used instead of the one offered by Hobson. Indeed, we agree with his later suggestion regarding the ability to be "moved" by another's attitudes. This develops apace within dyadic interaction within the first year and makes possible the development of triadic interaction (subject-interlocutor-object) and the capacity for symbolic thinking.

In the target article we place much stress on the epistemic triangle as a basis for development. We need to clarify its relation with triadic interaction following Meins's comments. What is referred to is interaction with other people about aspects of the world. It makes no difference if there are several other people involved - this would also be triadic in our sense. The point is to distinguish this form of interaction from forms of interaction in early infancy that are dyadic in that they involve the infant just interacting with the physical world or with a person. However, it is a misinterpretation by Wilson-Brune \& Woodward to assume that we believe that the development of infant's social knowledge only begins with triadic interaction. This would be a completely non-developmental account. The question of how infants develop the capacity for triadic interaction from dyadic interaction must be addressed (this issue was discussed in sect. 3.1 in our target article). Leekam points out that children with either autism or Williams syndrome have difficulties with dyadic interaction but for different reasons. Children with autism focus on dyadic interaction with objects, whereas children with Williams syndrome are more dyadically engaged with people. Both groups have difficulty with triadic interaction. By Leekam's analysis the problems identified in these syndromes are much more basic than at the "theory of mind" level (Leekam, in press).

Wilson-Brune \& Woodward suggest that our approach does not hold in the case of the emergence of pointing because infants' first pointing gestures may not be communicative but may instead index their own attention. Although there is much controversy about the origins of pointing, the evidence is consistent with our approach. We expect (as do Moore \& Corkum 1994) that infants first get into forms of interaction for other reasons and then learn within such interaction. For example, we would expect that infants' first pointing gestures would not be clearly social. As Wilson-Brune \& Woodward suggest (and also as stated in our article), there are clear developments in the sequence of pointing skills.

Thus, the lesson that we learn from our commentators in this section is that there is much to be gleaned from dyadic interaction in the first year of life, particularly in the level of affective engagement that the infant develops. We accept these ideas into our theory with thanks, especially as these commentaries remind us of the insights of parent-infant interaction studies in the 1970s and of Reddy's (e.g., 1991; 2003) impassioned critique of theory theory based in part on her rich descriptions of infant-parent interaction in the first year.

\section{R2.2. Language and criteria}

There are a number of points raised about our use of Wittgenstein's notion of criteria. These range from questions regarding word learning to the suggestion that the notion of criteria implies an enculturation position that we claimed to be avoiding.

The word learning problem raised by Miller \& Müller is that there are limitless possible meanings of new words, whether they are object labels or verbs. Thus, they suggest that the problems with word learning as correlating words with things also apply to correlating words with events. They also state that learning mental state terms would be particularly difficult because psychological states are not directly observable. To solve these problems they offer an interesting, if traditional, solution - that because the problem of mapping word to world is so intractable children may use 
Response/Carpendale \& Lewis: Constructing an understanding of mind

the structure of language as a cue to mental verb meaning. We do not deny that sentence structure is important in the child's language development in general and perhaps in learning the meaning of mental verbs. However, we have three responses to Miller \& Müller's analysis. First, in section 3.2 of our target article and here above we rehearse the Wittgensteinian argument that learning meanings of mental verbs is not a matter of naming inner unobservable states (Montgomery 2002), a view that is supported in a number of commentaries (Montgomery, Bickhard, Sokol \& Lalonde).

Second, we disagree with the assumptions about language that lead to setting up the problem of word learning in this way. Miller \& Müller appear to assume the constraints approach to the problem of word learning, according to which word learning involves creating and eliminating many possible hypotheses about how new words map onto the world (Tomasello 2001). The solution they suggest is that syntactic bootstrapping constrains the number of possible hypotheses. In contrast to this slightly disembedded solution, we endorse the social pragmatic approach to word learning described by Tomasello (2001), according to which children are "engaged in social interactions in which they are attempting to understand and interpret adult communicative intentions - so as to make sense of the current situation (Nelson 1985)" (Tomasello 2001, p. 113). We view meaning as related to the purposes for which words are used within interaction (Budwig et al. 2000; Turnbull \& Carpendale 1999a). Third, the structure of language may be important in children's learning the meaning of mental verbs, but the leap from syntax to meaning is still magical. ${ }^{3}$ It is not clear how the word learning problem is resolved by changing it to the unpacking of syntax (which is what Miller \& Müller seem to imply by referring to the "language structure") and mapping onto mental states. We endorse Budwig's suggestion to study constructions as the unit of analysis, because such a usage-based unit provides a far more credible route into meaning through language (Budwig 1998; Tomasello 1998).

Our suggestion to look beyond mental state terms to study talk about human activity is reflected on by Symons. He argues that we should not only look at parent-child discourse as a window on the process by which children acquire such terms, but we should also consider the child's spontaneous utterances during "solitary" tasks. This is an interesting idea that is compatible with our approach, and Symons considers this as an important potential route into an understanding of the child's internal mental states. From the unpublished study that he recounts, children's "discourse in solitary tasks" correlates highly with false belief understanding. Without more information about the task and whether the children were asked to narrate while performing it, we find it hard to judge whether Symons has identified a mechanism in keeping with Vygotsky's ideas. We agree that all children's activities, whether in discussion with adults, peers, siblings, or "alone," are useful markers of their constructions of mental state terms. Like Symons, our aim is to bridge the gap between parents'/others' mental state discourse and the child's constructions. However, we would add that it is not sufficient to correlate the frequency of mental state terms with test data. We agree with Budwig that it is important to examine how interactants use mental state terms for various purposes. In doing so the mechanism that Symons refers to retains its triadic struc- ture, within its interactional context. As we noted in our target article, words that are not considered mental state terms may still be important in talk about the psychological world. Although there is little research on this topic, Lohmann and Tomasello's (2003) language training study found no difference in training effect on false belief understanding between a condition in which mental state terms were used and a condition in which communication verbs such as "say" and "tell" were used.

Another commentary asks us to identify how children come to learn criteria for mental state terms. Barresi \& Moore are concerned that our theory potentially reduces to teacher-based enculturation. We feel that their depiction of language and criteria in our target article is incomplete because in section 3.1 we are at pains to identify the centrality of children's constructions of knowledge. This discussion was set into the framework of practices and criteria in section 3.2 to identify the dynamics of the epistemic triangle. In contrast to Barresi \& Moore, we suggest that our combined use of Piagetian theory and criteria protects us from an enculturation view. In addressing this criticism we refer to a distinction that Bickhard points out in his commentary, which is that, although children learn the meaning of words in terms of criteria, what is learned is not constituted by the criteria. Furthermore, it is not just that children learn culturally specific words for mental phenomena such as seeing; rather, these words are added to and become part of a practice. So, according to our position (and against a possibility considered by Barresi \& Moore), learning practices do not depend on a theory of mind; infants develop expectations about what will happen in certain situations in their practical, lived knowledge. For example, when a child learns to point declaratively to direct others' attention she is beginning to learn something about other people, the world, and how to coordinate her directedness toward aspects of the world with other people's directedness, or attention. Then, within interactions in which words are used to accompany pointing, she may add the word "look" to this shared practice. That is, this practice of pointing is one possible criterion for the use of the word "look." And, of course, the use of words is a constructive process, it cannot simply be social transmitted (see Nelson's comment about word use without meaning).

\section{R2.3. Structure and executive functions}

A third set of commentaries ask us to clarify the nature of the cognitive system that the child develops. Miller \& Müller argue that we need to describe the knowledge structures that characterize different levels of social understanding. The term "structure" is ambiguous because it can be interpreted as causally determining thinking or as a formal description of forms of thought (Chandler 1991; Chapman 1987b). We assume that Miller \& Müller would not mean it in the causal or functional sense but rather in the formal sense. However, in the list of theories they provide it is likely that a majority of theorists think of their structures as causal in the process of reasoning. In addition, one position that is missing, the theory theory, is assumed by Gopnik and Wellman (1992, p. 147) to be causal in the sense that the process of reasoning involves applying the laws of the theory. Another issue is whether structures are general across content. Most of the theorists listed by Miller $\&$ Müller would take this position; that is, once acquired, 
the structure would apply across content and in various circumstances. Our position, however, is that functionally distinct structures develop for each of the forms of joint attention. For example, pointings of different types (following pointing, following gaze, etc.) develop individually but these become reciprocally assimilated. That is, they are all activated by interacting with people and are combined gradually during social interactions.

In discussing the development of structure, Miller \& Müller quote Piaget (1967, p. 147): "Genesis emanates from a structure and culminates in another structure." There are suggestions in some other commentaries that go beyond our original analysis and illustrate just what is implied in this quotation. Fonagy is in broad agreement with our position and in drawing connections between Piaget and Bowlby (as does Symons from a critical perspective). But Fonagy also suggests other ways to think about the connections between attachment and social understanding. We agree with him (and Steele, who also mentions it) that an issue which we did not discuss is the role of attachment in emotional regulation. The structure of parent-child interaction plays a role in the child's emotional regulation, and this structure is gradually internalized into the patterns we see in classic attachment relationships. Fonagy also extends the same sort of argument to effortful control and inhibition, as abilities that develop within interpersonal engagement and are fostered by secure attachment relationships. We agree and, as we argue below, this fits nicely with our position that executive functioning is influenced by the child's history of social experience, not just an unfolding in maturation.

Fernyhough also wants us to specify the cognitive processes that result from developmental processes, whereas Gerrans asks us to specify the cognitive processes that lead to development. This reveals the greatly differing theoretical perspectives that have been put to us. Our approach is far closer to Chesnokova in her useful term "agency mediation" and to Fernyhough, who says that we explain how social competence develops but not what it is. Fernyhough is right that this is an aspect that we only hinted at and did not fully develop. He further wonders if the epistemic triangle is just a context for development or whether it, in addition, plays a role in children's reasoning about the mind. Fernyhough's discussion of Vygotsky is welcome and it is consistent with our position, developed from Chapman's theory. Chapman's position, drawing on Piaget, Vygotsky, and Gal'perin, is that the epistemic triangle is interiorized as a whole:

interiorized communicative exchange between the subject and the interiorized interlocutor is the origin of reflective awareness. The coordination of cognitive operations by the subject and the interlocutor is the source of the "operational compositions" that characterize concrete operations. Such coordinations are the interiorized counterpart of interpersonal cooperation. (Chapman 1991, p. 219)

Piaget (1962/2000) agreed with Vygotsky's view that reflective thought arises due to the interiorization of egocentric speech. Thus, in response to Miller \& Müller, development "emanates" from the structure of triadic interaction which includes the structures that the child brings to the interaction, and it "culminates" in the interiorized structure of the epistemic triangle. As skills become consolidated, further levels of the structure involve the ability to reflect upon this interiorised epistemic triangle. These develop in a local, piecemeal way, not - as hinted at by Miller \& Müller - by the application of a new rule or representation system.

The issue of structure has been made more complex by recent work, which links social development with what are taken to be domain general skills under the banner of executive functions. Hala suggests that our position is compatible with executive function accounts. We agree that an aspect of our approach that needs elaboration is in relation to the notion of executive functions, and in our target article we could only acknowledge that in a brief note. Two key issues are raised. First, as has often been pointed out (Monsell 1996; Rabbitt 1997; Towse et al. 2000), the notion of executive functions is complex and ill-defined even in adults, so we must be immediately cautious about describing individual skills or the ragbag of "executive" skills as given. However, recent structural equation modeling analyses suggest that in both adults (Miyake et al. 2000) and children (Shimmon et al. 2003) there is distinctiveness between areas of executive test performance (e.g., working memory is distinct from set shifting), but individual constructs like these fit best into a unified latent variable "executive function." However, performance on test batteries does not reveal conceptual links between, or a developmental model of, such skills. An advantage of an activity-based approach is that we do not have to think of something like "attention" or "attention-shifting" as independent characteristics of the child which the child brings to the interaction. Instead the child's "attentiveness" may well be influenced by the interaction of the dyad (Hobson, in press).

Therefore, second, we do not think it is helpful to assume that executive functions simply unfold with maturation. Rather, we agree with Hala that the development of these particular skills is likely to be complex, embedded within the nature of children's experiences. She points out that there might be a deeper relation between executive skills and different aspects of social understanding and that there is a bidirectional relationship between the child and other people. We would take the analysis further to examine a number of other bidirectional patterns of influence within the epistemic triangle - including interactions between each executive skill. It is these links that we take to be the more complex structures that are developing in the child and which are hinted at in the research cited by Hala. Although we acknowledge that such ideas are in need of supportive evidence, we note recent data which show that social interaction (maternal verbal scaffolding of the child) at age 3 years is linked with the child's language at age 4 and search retrieval skills at age 6 (Landry et al. 2002). Such findings suggest that the development of executive skills is embedded in the child's social experiences.

\section{R3. Should the theory be extended?}

Several commentators have offered suggestions about how our theory can be improved. In our response on infancy, above, we agreed that an understanding of social development must have as its starting point the dyadic exchanges between individuals. One of the points that we understated in the target article was that much of this interaction is highly affectively laden and what the child constructs within the interaction is charged with emotion. In the main, the commentaries discussed in this section reflect upon the ways in which the theory we present can be improved by 
embedding the epistemic triangle within a wider and more enriched social process - the reason why we are prepared to refer to it as the epistemic-relational triangle. The target article suggests that this framework is needed in order to understand the dynamic between the subject-interlocutor and subject-object interaction involved in the development of knowledge. We admit that we couched our analysis in a way that enabled an exchange and critique of the traditional approaches in this area that we have just discussed - approaches in which mental states are segregated from anything that remotely resembles human experience. The commentaries we discuss in this section mainly point out that there is more to the development of social knowledge than the emergence of the child's ability to reflect on cognitive mental states like beliefs. These other aspects of development hinge on issues that we hinted at in the target article, surrounding the nature and importance of affective engagement both in infancy and, indeed, all human social understanding. We welcome such a move, as long as the dynamic of subject-interlocutor and subject-object is kept in place.

\section{R3.1. The role of relationships in social development: Let's get emotional}

We are impressed by the ways in which several of the commentators extend our analysis of the role of the individual's constructions within interactions into specific types of relationships such as siblings and peers, and the importance of affect engagement, particularly in attachment relationships and emotional relatedness. However, as a result of comments from Gerrans, Meins, and Steele, we first need to clarify the relational position we endorse.

Gerrans seems to think that rejecting individualism means we cannot talk about the characteristics of individuals, like those with autism. To the contrary, rejecting individualism does not mean that we ignore the fact that individuals vary in the characteristics they bring to interaction. McGeer asks us to clarify that a rejection of individualism does not imply rejecting internalism. We agree. We do not just focus on the individual or the social. Relationism or systemism takes the strengths of both individualism and collectivism. Our "mechanism" is the internalization of action and interaction. That is, our explanation for the development of social understanding cannot be individualistic because it depends on the relations between people. But neither is it holistic because obviously individuals interact and bring their characteristics to that interaction. Gerrans is right that children with disorders such as autism have some neurological impairment. However, the whole story cannot be told on the basis of the individual. Instead, the relations between people are essential, because children with other difficulties such as deafness or severe social deprivation may also develop an autism-like syndrome (Hobson 2002).

In contrast to Gerrans, Meins claims that we focus too much on the child and that "the child's attributes may initially be rather unimportant." We should not focus on either the child or the other person; rather it is the relations between them that are important. Of course, both interactants will influence those relations due to their characteristics. The claim that individual differences in caregivers are important is embedded in our position, and therefore we do not assume that all mother-infant and child-sibling relationships are the same, contrary to Meins's reading. Meins is right that the effects of siblings and of parenting style have been found only with preschoolers but this reflects the nature of the available data, not necessarily that we are trying to explain the wrong evidence. Within our framework it is important to examine the nature of sibling relationships (the quality of interaction, etc.), not just whether or not the child has a sibling.

Meins notes that we mention the link between attachment security and social understanding and implies that we believe this is a direct link. In fact, we mention this as evidence that requires an explanation, not as an explanation itself. However, we do clearly agree with Meins that mere exposure to mental state language is not enough for children's social development. What she calls for in her final paragraph regarding an integrated system is just what we mean by a focus on relations.

Howe is in agreement with our position and suggests extensions by further focusing on children's sibling relationships, their friendships, and their pretend play. We agree, and further suggest that in such extensions we need a theory with which to make sense of various forms of interaction. We wanted to make it clear that the Piagetian distinction between constraining and cooperative relationships was originally introduced by Piaget (1932/1965b, 1977/ 1995) by contrasting parent and peer relations. Steele views Piaget's distinction between constraining and cooperative relationships as a "dichotomous model." However, the target article states clearly that Piaget's distinction refers to a continuous model because any relationship (i.e., parent-child, siblings, and peers) is some mixture of these two types.

We acknowledge Steele's claim that other aspects of social relationships should be considered to capture the complexities of human relationships. Some of his suggestions appear to refer to the content of discussion. In our article we recognize the importance of both what is being talked about and the influence of the relationship on the child's understanding of that talk. Steele extends this analysis to describe the types of attachment relationship, but not explicitly to the processes that constitute such types. We feel that a more profitable extension of our analysis within attachment theory would be to follow the suggestion of Fonagy and Thompson \& Raikes, who focus more closely upon the dynamics of attachment and emotional relationships. Both these perspectives are constructive, affective additions to the approach we propose. We feel that, just as cognitively based models like ours need an emotional dimension provided by these theoretical perspectives, attachment-based accounts also need an analysis of triadic interaction in our sense, particularly into which language is integrated. So we hope that the argument is more "new" than Steele suggests.

We also concur with Banerjee who discusses the role of social experience, or self-regulation, in advanced social understanding, such as the role of peer interactions in learning about interpretation. Zerwas, Balaraman \& Brownell (Zerwas et al.) provide an elaboration of the role of peers in social development and the special challenges and opportunities afforded by peers, involving opportunities for cooperation as well as conflict. The role of conflict is also discussed by Howe and Thompson \& Raikes. At least one important aspect of conflict between mothers and their children concerns how mothers deal with and talk about inevitable conflicts that occur with young 
toddlers (Laible \& Thompson 2002). This line of evidence fits with the evidence reviewed in our target article.

Most of the commentaries discussed in this section suggest that we should extend our framework to include different types of social relationships. Two commentators (Nelson, Vinden) feel that an understanding of such relationships can be achieved only within a wider cultural framework and that we did not go far enough in this direction. Vinden advances a constructivistic view of enculturation, albeit using terminology slightly different from ours. We do not disagree with her suggestion. Our purpose was to distinguish our position of construction and reconstruction from a view of passive socialization or the transmission of knowledge. We do not dismiss the importance of culture. We agree that the child will influence the relationship with her caregivers, but we do not think that enculturation is the right word for this, given that it is often taken to consist of the passive passing on of beliefs, conventions, and so on, from one generation to the next. Nelson also thinks that we did not go far enough in the cultural-historical direction. We agree that such an approach should be taken and we believe that ours could easily be expanded in that direction. However, we would emphasize that "culture" should not be reified. It is created and recreated by interactants within everyday interactions (Berger \& Luckmann 1966), thus the epistemic triangle is a means of understanding the dynamic between individual action and everyday practices. In the next section we elaborate on further possible developments of our theory raised in the commentaries.

\section{R3.2. Broadening our conception of social understanding: Let's get more emotional (and normative)}

In this section we add four perspectives from the commentators which are consistent with and further extend our theory. First, we must broaden the age span examined in the target article, as suggested by Mitchell. Banerjee and Jenkins \& Oatley helpfully list further development in social understanding beyond five years of age. We want to emphasize our strong agreement that our list of issues beyond false belief needs to be extended, because Lourenço appeared to have the impression that we might be thinking that development in social understanding stops at age eight years with the beginnings of an interpretative understanding of knowledge. Jenkins and Oatley provide examples of distributed cognition within the crew of a navy ship to hint at the use of the epistemic triangle in adult learning, as each specialist tries to negotiate meaning with his or her colleague. Their term "prioritization of joint goals" can be applied well after age eight years. Banerjee further broadens the conceptualization of social understanding to include irony (see also Yasui 2003), self-presentational motives, and emotions such as embarrassment in a wider variety of relationships, particularly with peers. To this broadening of how we think about social understanding and social competence we could add the development of competence in social interaction, such as politeness (Brown \& Levinson 1987; Turnbull 2003). Our theory provides a framework with which to incorporate these extensions. Only if developmental psychology extends the focus of the field in directions like these will we be able to address McGeer's question of what is included in "social understanding." We agree with her suggestion that what is developing is not just a body of knowl- edge, but rather a set of practices and that this involves the development of social agency and the ability to interact within a community. The issue of agency is also hinted at by Sokol \& Lalonde (see Sokol \& Chandler 2003). Russell (1996) has identified the depth of the role of agency in the development of knowledge, but he downplays analysis of its social dimension.

The second issue concerns the extension of our framework to include other aspects of cognition, not usually linked to a social dimension. Smith questions whether we can account for the development of normativity from the perspective of the epistemic triangle. We recognize that the issue of normativity, or how we get from the causal conditions of knowledge to normative knowledge, is a neglected problem in developmental psychology, and it is also a test of an adequate theory of development. We did not deal with the issue of normativity, but we believe that our approach could be extended to do so for the reason that our position is based on Piagetian ideas about the development of knowledge. (Note that the computational approach advocated by Gerrans and the neuro-reductive approach championed by Craig \& Barrett cannot recognize normativity as a topic of study.) As Smith (1993) argues, one of the goals of Piaget's genetic (i.e., developmental) epistemology was to ground normativity in the natural world - that is, to "show how to secure a place for the normative within the natural" (Kitchener 2004, p. 61). In Kitchener's words, Piaget's solution to this problem was that "epistemic norms emerge from certain kind of social relations between individuals - those involving cooperation" (p. 62). Therefore, a necessary component for normativity is social interaction of particular types. By this token, all of Smith's examples of normativity are rooted in social interaction because they are justifications. We suggest that an act of judgment does not exist outside of human practices. For example, Smith's case [E] appears to be an instance of the epistemic triangle in action.

Third, several commentators argue that we need to include emotions more fully (Banerjee, Fonagy, Hobson, Izard, Thompson \& Raikes). We agree with Izard that there are a number of ways in which our account could be further extended to integrate the role of emotions. She points out many areas of literature that could enrich our perspective. As we argue below, with reference to a similar point made by Fonagy, such additions will more fully incorporate the role of emotions in social development beginning in infancy, and they are entirely consistent with our approach. Like Izard, we would avoid any hard and fast line between cognition and emotion because many emotions cannot be simply separated from cognition, as in surprise, envy, and jealousy. Second, as Izard suggests, emotions may play a role in influencing the relationships young children engage in, which in turn may influence the development of their social understanding. Such emotion may be partially attributed to the child's characteristics but must take into account the interaction between the child and the other person. We agree with Banerjee that the notion of self-regulation provides a crucial link with our own framework for understanding the child's internalization of the epistemic triangle. From our perspective it follows that children learn about emotions in the same way that they learn about beliefs and intentions. Finally, Thompson \& Raikes extend the need to study emotional development in children's early understanding of mental states within a broader attachment framework. 
Response/Carpendale \& Lewis: Constructing an understanding of mind

The fourth area of research suggested in the commentaries is a focus upon more intricate details of the child's conversations with others (Astington, Nelson, Siegal, Thompson \& Raikes, Walker-Andrews \& Hudson). We find Nelson's label of "collaborative construction" helpful as it places the child's developing understanding within a network of social relationships and the ebb and flow of everyday discourse. It provides clear guidelines for how research on children's understanding of the mind should be conducted. This is exemplified in the research areas described by Walker-Andrews \& Hudson: We agree that the work they review on emotion perception, pretence understanding, event knowledge, and autobiographical memory will add to the richness of our account of mental state understanding. Thompson \& Raikes suggest that we neglect several explicit and implicit features of conversational discourse such as provocative questions, elaborative detail, and mothers' reference to emotions. We agree that it is important to consider other aspects of relationships beyond cooperation and constraint, as well as to examine cooperative interaction in concrete detail. However, we also feel that when considering factors such as these, the notion of cooperation may be useful because such forms of discourse may facilitate development more in cooperative relationships than in constraining relationships.

Astington makes the interesting suggestion that the child's linguistic ability and experience in social and communicative interaction may play different roles in social development, although she acknowledges that the two factors are related. However, in the view we take of language as activity, it would seem to be very hard conceptually to separate such factors. There are also potential empirical problems with the way theory of mind data are collected, which we refer to in the target article's concluding section. To use Astington's research examples, we need to know about the quality of interactions of deaf children with hearing parents, and the parent-child dyads in hearing children with no siblings but high language ability, in order to reveal the possible reasons why these children appear to be different in their false belief performance compared to other groups. Nevertheless, Astington's commentary underlines the importance of analyzing language and communication in detail, as well as our conceptions of language (Budwig, Nelson). The important issue is that reference to "conversation" alone is not sufficient either to undermine our position or to explain the influence of interaction on cognition. Indeed, it can lead to the problems that are inherent in Siegal's account. He appears to suggest that (1) the child's theory of mind is situated within her or his cognitive architecture, and (2) the false belief performance is essentially an understanding of conversation. The fact that Siegal offers no definition of "conversation" stands in contrast to the accounts of Nelson and Thompson \& Raikes. His description offers little in the way in which the architecture develops or how the term conversation helps in his analysis. The target article and many commentaries attempt to grapple with these links.

\section{R4. Feeling our way forward}

We expected more criticism of both our use of a Wittgensteinian analysis of word-world relations and the blend with a mix of Piagetian and Vygotskian constructivism. We are slightly puzzled by the relative lack of explicit critiques of either these individual theories or how we put them together. We see this cocktail as providing a fundamental critique of the study of social development, or indeed any developmental or cognitive theory, in which meaning is slipped in before the theory is articulated. We welcome further discussion on this issue because we agree with Nelson that the implications are potentially profound.

The stumbling block for many of our commentators was the lack of a "mechanism" in our theory. One implication of the theoretical origins of our approach is that it is activitybased and grounded in practices, not internal representations. Structure arises as a result of the process. Thus, the mechanism and the process are the same, and each is based on the child's constructions of social interactions, where meanings are negotiated. We realize that this unsettles some commentators but it follows naturally from our critique of their assumptions. The implications for research are first that we need more (not less) neurotic task fixation (although not just on false belief tasks), in which we try to work out how children construct meaning in a wider range of settings and tasks beyond false belief. Second, we need to conduct research on the development of children's shared practices upon which mental state understanding develops. The studies of Judy Dunn and the ideas outlined in the conclusion to our target article provide examples.

It was clear from the commentaries that we need to extend the theory, and most of the commentators felt that this was a relatively straightforward task. Although we need to add affective engagement in infancy and beyond, we agree with many (e.g., Banerjee, Fonagy, Izard, Steele) that cognition and emotion should not be completely separated - both are aspects of the individual's reaction to and constructions of events. But we maintain that the epistemic triangle is a vital formulation for understanding how individuals develop knowledge and it adds to the affective engagements described by many, including those from an attachment perspective. We have not described how our framework might work at higher levels of the structure, and we need to go further in explaining the development of structure by providing an account of how the epistemic triangle within particular domains of thought itself becomes an object of reflection.

We thank the referees of the original submission, the editors at $B B S$, and the commentators for their work, their challenges, and their constructive criticism. They have forced us to assimilate some points and painfully to accommodate others, thus reassuring us that the constructive process continues in development. As Astington's commentary suggests, it seems that the field is changing. We look forward to a further consolidation of socially grounded theory of children's social understanding.

\section{NOTES}

1. Lourenço also suggests that our theory is not necessary because it does not go beyond theories such as Selman's (1980). Selman's theory is compatible with our position, but there are some important differences. Selman already assumes much of what we are trying to explain because he does not provide an account of infant social development. Furthermore he does not account for the role of language in social development. What he does do well is provide a description of the levels of complexity of different situations of social understanding, and integrating his theory could potentially extend our position.

Lourenço also asserts that we claimed to differ from Thelen and Smith because of the "ideas of interactionism and transac- 
tionalism." In fact, we did not say this. Rather, in Note 3 in our target article we state that although we have much in common with dynamic systems theories we appear to differ from Thelen et al. (2001) in the starting point in infant development. That is, they seem to assume objectivity rather than explain it (Müller \& Carpendale 2001).

2. We refer anyone still convinced of fundamental differences between Piaget and Vygotsky to Smith's (1996) test of 10 statements from each of these theorists, which stump even experts (see also Amin \& Valsiner 2004).

3. Miller \& Müller note that in other work (Turnbull \& Carpendale 1999a) we reject the code model of language (i.e., the view that language works by encoding meaning in words and utterances that are then transmitted to others for decoding) and they argue that the "language code" is also important. Our reasons for rejecting the code model is that the same word or utterance can be used to convey different meanings when used within different contexts of ongoing sequences of interaction (Turnbull \& Carpendale 1999a). That is, as Budwig notes, language is indexical (e.g., Budwig et al. 2000; Suchman 1987; Turnbull 2003). This suggests that rather than thinking of language as having fixed meanings attached to representations (the view of language that Wittgenstein argued against), we should think of language as a means for directing and coordinating attention with others within shared social settings (Tomasello 2001; 2003). Miller and Müller are right that a word such as "think" can be used in many different ways, in many different language games. From this we take the point that we need to describe in more concrete detail how children might learn about the many ways the word "think" can be used.

\section{References}

Letters " $a$ " and " $r$ " appearing before authors' initials refer to target article and response, respectively.

Abe, J. A. \& Izard, C. E. (1999) A longitudinal study of emotion expression and personality relations in early development. Journal of Personality and Social Psychology 77:566-77. [CEI]

Adolphs, R. (2003) Cognitive neuroscience of human social behaviour. Nature Reviews 4:165-78. [PF]

Ainsworth, M. D., Blehar, M. P., Waters, E. \& Wall, S., eds. (1978) Patterns of attachment: A psychological study of the strange situation. Erlbaum. [CEI, HS]

Amin, T. G. \& Valsiner, J. (2004) Coordinating operative and figurative knowledge: Piaget, Vygotsky, and beyond. In: Social interaction and the development of knowledge, ed. J. I. M. Carpendale \& U. Müller. Erlbaum. [rJIMC]

Appleton, M. \& Reddy, V. (1996) Teaching three year-olds to pass false belief tests: A conversational approach. Social Development 5:275-91. [DKS]

Arnsten, A. F. T. (1998) The biology of being frazzled. Science 280:1711-12. [PF]

Arnsten, A. F. T., Mathew, R., Ubriani, R., Taylor, J. R. \& Li, B.-M. (1999) Alpha-1 noradrenergic receptor stimulation impairs prefrontal cortical cognitive function. Biological Psychiatry 45:26-31. [PF]

Astington, J. W. (1996) What is theoretical about the child's theory of mind? A Vygotskian view of its development. In: Theories of theories of mind, ed. P. Carruthers \& P. K. Smith, pp. 184-99. Cambridge University Press. [JWA, aJIMC]

(2000) Language and metalanguage in children's understanding of mind. In Minds in the making: Essays in honor of David R. Olson, ed. J. W. Astington. Blackwell. [aJIMC]

(2001) The future of theory-of-mind research: Understanding motivational states, the role of language, and real-world consequences. Child Development 72:685-87. [aJIMC]

Astington, J. W. \& Baird, J. A., eds. (in press) Why language matters for theory of mind. Oxford University Press. [aJIMC]

Astington, J. W. \& Gopnik, A. (1991) Theoretical explanations of children's understanding of mind. British Journal of Developmental Psychology 9:7-29. [aJIMC]

Astington, J. W., Harris, P. L. \& Olson, D. R., eds. (1988) Developing theories of mind. Cambridge University Press. [aJIMC]

Astington, J. W. \& Jenkins, J. M. (1995) Theory of mind development and social understanding. Cognition and Emotion 9:151-65. [aJIMC, JMJ]
(1999) A longitudinal study of the relations between language and theoryof-mind development. Developmental Psychology 35:1311-20. [aJIMC, JMJ]

Astington, J. W. \& Olson, D. R. (1995) The cognitive revolution in children's understanding of mind. Human Development 38:179-89. [JWA, aJIMC, OC, OML]

Bakeman, R. \& Adamson, L. (1986) Infants' conventionalized acts: Gestures and words with mothers and peers. Infant Behavior and Development 9:215-30. [CW-B]

Baker, G. P. \& Hacker, P. M. S. (1984) Language, sense and nonsense. Blackwell. [aJIMC]

Bakti, A., Baron-Cohen, S., Wheelwright, S., Connellan, J. \& Ahluwalia, J. (2000) Is there an innate gaze module? Evidence from human neonates. Infant Behavior and Development 23:223-29. [TR]

Balaraman, G., Brownell, C. A. \& Campbell, S. B. (2003) Self-regulation and peer social competence in the preschool years (under review). [SZ]

Baldwin, D. A. (1995) Understanding the link between joint attention and language. In: Joint attention: Its origins and role in development, ed. C. Moore \& P. J. Dunham. Erlbaum. [aJIMC]

Baldwin, D. A., Baird, J. A., Saylor, M. M. \& Clark, M. A. (2001) Infants parse dynamic action. Child Development 72:708-17. [CW-B]

Baldwin, D. A. \& Moses, L. J. (1996) The ontogeny of social information gathering. Child Development 67:1915-33. [aJIMC,VM]

Baldwin, J. M. (1906) Thoughts and things. Vol. 1, Functional logic. MacMillan. [aJIMC]

Banerjee, R. (2002a) Children's understanding of self-presentational behaviour Links with mental-state reasoning and the attribution of embarrassment. Merrill-Palmer Quarterly 48:378-404. [RB]

(2002b) Audience effects on self-presentation in childhood. Social Development 11:487-507. [RB]

Banerjee, R. \& Yuill, N. (1999) Children's explanations for self-presentational behaviour. European Journal of Social Psychology 29:105-11. [RB]

Baron-Cohen, S. (1995) Mindblindness: An essay on autism and theory of mind. MIT Press. [aJIMC]

Baron-Cohen, S., O’Riordan, M., Stone, V., Jones, R. \& Plaisted, K. (1999) Recognition of faux pas by normally developing children and children with Asperger Syndrome or high-functioning autism. Journal of Autism and Developmental Disorders 29:407-18. [RB]

Barreau, S. \& Morton, J. (1999) Pulling smarties out of a bag: A Headed Records analysis of children's recall of their own past beliefs. Cognition 73:65-87. [TPG]

Barresi, J. \& Moore, C. (1993) Sharing a perspective precedes the understanding of that perspective. Behavioral and Brain Sciences 16:513-14. [JB]

(1996) Intentional relations and social understanding. Behavioral and Brain Sciences 19(1):107-54. [JB, aJIMC, CW-B]

Bartsch, K. (2002) The role of experience in children's developing folk epistemology: Review and analysis from the theory-theory perspective. New Ideas in Psychology 20:145-61. [KB]

Bartsch, K., Campbell, M. D., Jefferies, J. \& Wright, J. (2003) Children's use of belief information in interactive persuasion tasks. Poster session presented at the Biennial Meeting of the Society for Research in Child Development, Tampa, FL, April 2003. [KB]

Bartsch, K. \& Estes, D. (1993) Are false beliefs representative mental states? Behavioral and Brain Sciences 16:30-31. [KB]

Bartsch, K. \& London, K. (2000) Children's use of belief information in selecting persuasive arguments. Developmental Psychology 36:352-65. [KB]

Bartsch, K. \& Wellman, H. M. (1995) Children talk about the mind. Oxford University Press. [KB, aJIMC, DEM]

Bates, E., Benigni, L., Bretherton, I., Camaioni, L. \& Volterra, V. (1979) The emergence of symbols: Cognition and communication in infancy. Academic Press. [CW-B]

Bates, E., Camaioni, L. \& Volterra, V. (1976) Sensorimotor performatives. In: Language and context, ed. E. Bates. Academic Press. [aJIMC]

Baumrind, D. (1991) Parenting styles and adolescent development. In Encyclopedia of adolescence, vol. 2, ed. R. M. Lerner, A. C. Petersen \& J Brooks-Gunn. Garland. [aJIMC]

Beebe, B. \& Lachmann, F. (2002) Infant research and adult treatment: Coconstructing interactions. Analytic Press. [HS]

Bell, R. Q. (1968) A reinterpretation of the direction of effects in studies of socialization. Psychological Review 75:81-95. [aJIMC]

Bereiter, C. (2001) Education and mind in the knowledge age. Erlbaum. [LS]

Berger, P. L. \& Luckmann, T. (1966) The social construction of reality: A treatise in the sociology of knowledge. Anchor Books. [rJIMC]

Bickhard, M. H. (1998) Levels of representationality. Journal of Experimental and Theoretical Artificial Intelligence 10(2):179-215. [MHB]

(2001) Why children don't have to solve the frame problems: Cognitive representations are not encodings. Developmental Review 21:224-62. [MHB] 
References/Carpendale \& Lewis: Constructing an understanding of mind

(2003) Some notes on internal and external relations and representation. Consciousness and Emotion 4:101-10. [MHB]

(2004) The social ontology of persons. In: Social interaction and the development of knowledge, ed. J. I. M. Carpendale \& U. Müller. Erlbaum. [rJIMC]

Bickhard, M. H. \& Campbell, R. L. (1989) Interactivism and genetic epistemology. Archives de Psychologie 57(221):99-121. [MHB]

Blair, R. J. \& Cipolotti, L. (2000) Impaired social response reversal. A case of "acquired sociopathy." Brain 123(Pt 6):1122-41. [PF]

Bloom, P. (2001) Controversies in the study of word learning. Behavioral and Brain Sciences 24:1124-34. [MS]

Bloom, P. \& German, T. (2000) Two reasons to abandon the false belief task as a test of theory of mind. Cognition 77:B25-B31. [TPG, MS]

Bogdan, R. J. (1997) Interpreting minds. MIT Press. [PF]

Boom, J. (2004) Individualism and collectivism: A dynamic systems interpretation of Piaget's interactionism. In: Social interaction and the development of knowledge, ed. J. I. M. Carpendale \& U. Müller. Erlbaum. [aJIMC]

Bowlby, J. (1969/2000) Attachment and loss, vol. 1, Attachment. Basic Books. [HS]

(1971) Attachment and loss, vol. 1, Attachment. Hogarth Press (reprinted by Penguin, 1978). [JMJ]

(1980) Attachment and loss, vol. 3: Loss: Sadness and depression. Hogarth Press and Institute of Psycho-Analysis. [PF]

Boyes, M., Giordano, R. \& Pool, M. (1997) Internalization of social discourse: A Vygotskian account of the development of young children's theories of mind. In: Sociogenetic perspectives on internalization, ed. B. D. Cox \& C. Lightfoot, pp. 189-202. Erlbaum. [JWA]

Bretherton, I. (1991) Intentional communication and the development of an understanding of mind. In: Children's theories of mind: Mental states and social understanding, ed. D. Frye \& C. Moore. Erlbaum. [aJIMC]

(1998) Attachment and psychoanalysis: A reunion in progress. Social Development 7:132-36. [HS]

Bretherton, I., Bates, E., Benigni, L., Camaioni, L. \& Volterra, V. (1979) Relations between cognition, communication, and quality of attachment. In: The emergence of symbols: Cognition and communication in infancy, ed. E. Bates. Academic Press. [aJIMC]

Bretherton, I., McNew, S. \& Beeghly-Smith, M. (1981) Early person knowledge as expressed in gestural and verbal communication: When do infants acquire a "theory of mind"? In: Infant social cognition: Empirical and theoretical considerations, ed. M. E. Lamb \& L. R. Sherrod. Erlbaum. [aJIMC]

Bretherton, I. \& Munholland, K. (1999) Internal working models in attachment relationships: A construct revisited. In: Handbook of attachment: Theory, research, and clinical applications, ed. J. Cassidy \& P. Shaver, pp. 89-111. Guilford Press. [RAT]

Bronfenbrenner, U. (1979) The ecology of human development. Harvard University Press. [HS]

(1989) Ecological systems theory. In: Annals of child development, vol. 6, ed. R. Vasta, pp. 187-251. JAI Press. [SH]

Brown, J. R., Donelan-McCall, N. \& Dunn, J. (1996) Why talk about mental states? The significance of children's conversations with friends, siblings, and mothers. Child Development 67:836-84. [aJIMC, EM, SZ]

Brown, J. R. \& Dunn, J. (1992) Talk with your mother or your sibling? Developmental changes in early family conversations about feelings. Child Development 63:336-49. [TR]

Brown, P. \& Levinson, S. (1987) Politeness: Some universals in language usage Cambridge University Press. [rJIMC]

Brown, T. (2003) Reductionism and the circle of sciences. In: Reductionism and the development of knowledge, ed. T. Brown \& L. Smith, pp. 3-26. Erlbaum. [rJIMC]

Brownell, C. A., Balaraman, G., Zerwas, S., Mariaskin, A. \& Kimmel, A. (2003) Peer social skill and social understanding in toddlers: Joint play, joint attention, and cooperative problem-solving. Poster session presented at the biennial meeting of the Society for Research in Child Development, Tampa, FL, April 2003. [SZ]

Brownell, C. A. \& Brown, E. (1992) Peers and play in infants and toddlers. In: Handbook of social development, ed. V. Van Hasselt \& M. Hersen, pp. 183 200. Plenum Press. [SZ]

Brownell, C. A. \& Carriger, M. S. (1990) Changes in cooperation and self-other differentiation during the second year. Child Development 61:1164-74. [SZ]

Bruner, J. (1986) Actual minds, possible worlds. Harvard University Press. [DKS] (1990) Acts of meaning. Harvard University Press. [aJIMC]

Budwig, N. (1998) How far does a construction grammar approach to argument structure take us in understanding children's language development? Journal of Child Language 25:443-47. [NB, rJIMC]

(2002) A developmental-functionalist approach to mental state talk. In: Language, literacy, and cognitive development: The development and consequences of symbolic communication, ed. E. Amsel \& J. P. Byrnes, pp. 59-86. Erlbaum. [NB, aJIMC]

(2003) The role of language in human development. In: Handbook of developmental psychology, ed. J. Valsiner \& K. Connolly, pp. 217-37. Sage. [NB]

Budwig, N., Stein, S. \& O'Brien, C. (2001) Nonagent subjects in early child language: A crosslinguistic comparison. In: Children's language: Interactional contributions to language development, ed. K. Nelson, A. Aksu-Koc \& C. Johnson, pp. 49-67. Erlbaum. [NB]

Budwig N., Wertsch, J. V. \& Uzgiris, I. C. (2000) Communication, meaning, and development: Interdisciplinary perspectives. In: Communication: An arena of development, ed. N. Budwig, I. C. Uzgiris \& J. V. Wertsch, pp. 1-14. Ablex. [rJIMC]

Bunge, M. (2000) Ten modes of individualism - none of which works - and their alternatives. Philosophy of the Social Sciences 30:384-406. [aJIMC]

Call, J. \& Tomasello, M. (1999) A nonverbal false belief task: The performance of children and great apes. Child Development 70:381-95. [aJIMC]

Campbell, R. L. \& Bickhard, M. H. (1993) Knowing levels and the child's understanding of mind. Behavioral and Brain Sciences 16:33-34. [aJIMC]

Canfield, J. V. (1993) The living language: Wittgenstein and the empirical study of communication. Language Sciences 15:165-93. [aJIMC]

(1999) Folk psychology versus philosophical anthropology. Idealistic Studies 3:153-71. [aJIMC]

Cantor, N. \& Kihlstrom, J. (1989) Social intelligence and cognitive assessment of personality. In: Advance in social cognition, vol. 2, ed. R. Wyer \& T. Srull. Erlbaum. [OC]

Carlson, S. M. \& Moses, L. J. (2001) Individual differences in inhibitory control and children's theory of mind. Child Development 72:1032-53. [PF, SH, CAM]

Carlson, S. M., Moses, L. J. \& Breton, C. (2002) How specific is the relation between executive function and theory of mind? Contributions of inhibitory control and working memory. Infant and Child Development 11:73-92. [SH]

Carlson, S. M., Moses, L. J. \& Hix, H. R. (1998) The role of inhibitory control in young children's difficulties with deception and false belief. Child Development 69:672-91. [TPG, SH]

Carpendale, J. I. M. (1999a) Constructivism, communication, and cooperation: Implications of Michael Chapman's "Epistemic Triangle." In: The development of representational thought: Theoretical perspectives, ed. I. Sigel. Erlbaum. [aJIMC]

(1999b) Symbols and side effects: Commentary on "Cognitive development as an executive process." Developmental Science 2:279-80. [aJIMC]

(2000) Kohlberg and Piaget on stages and moral reasoning. Developmental Review 20:181-205. [aJIMC]

Carpendale, J. I. M. \& Chandler, M. J. (1996) On the distinction between false belief understanding and subscribing to an interpretive theory of mind. Child Development 67:1686-706. [RB, aJIMC]

Carpendale, J. I. M., McBride, M. \& Chapman, M. (1996) Language and operations in children's class reasoning: The operational semantic theory of reasoning. Developmental Review 16:391-415. [arJIMC]

Carpendale, J. I. M. \& Müller, U. (2004) Social interaction and the development of rationality and morality. In: Social interaction and the development of knowledge, ed. J. I. M. Carpendale \& U. Müller. Erlbaum. [aJIMC]

Carpenter, M., Call, J. \& Tomasello, M. (2002) A new false belief test for 36month-olds. British Journal of Developmental Psychology 20:393-420. [aJIMC]

Carpenter, M., Nagell, K. \& Tomasello, M. (1998) Social cognition, joint attention, and communicative competence from 9 to 15 months of age. Monographs of the Society for Research in Child Development 63 (Issue No. 4, Serial No. 255):1-176. [aJIMC, CW-B]

Carruthers, P. (2003) The cognitive functions of language. Behavioral and Brain Sciences 25:657-726. [MS]

Carruthers, P. \& Smith, P. K., eds. (1996) Theories of theories of mind. Cambridge University Press. [aJIMC]

Chandler, M. J. (1988) Doubt and developing theories of mind. In: Developing theories of mind, ed. J. W. Astington, P. L. Harris \& D. R. Olson. Cambridge University Press. [aJIMC]

(1991) Alternative readings of the competence-performance relation. In: Criteria for competence, ed. M. J. Chandler \& M. Chapman, pp. 5-18. Erlbaum. [arJIMC]

(1997) Stumping for progress in a post-modern world. In: Change and development: Issues of theory, method, and application, ed. E. Amsel \& K. A Renninger. Erlbaum. [aJIMC]

(2001) Perspective taking in the aftermath of theory-theory and the collapse of the social role-taking literature. In: Working with Piaget: In memoriam Barbel Inhelder, ed. A. Tryphon \& J. Voneche. Psychology Press. [aJIMC]

Chandler, M. J. \& Carpendale, J. I. M. (1998) Inching toward a mature theory of mind. In: Self-awareness: Its nature and development, ed. M. Ferrari \& R. J. Sternberg. Guilford Press. [aJIMC]

Chandler, M. J. \& Hala, S. (1994) The role of personal involvement in the assessment of early false belief skills. In: Children's early understanding of 
mind: Origins and development, ed. C. Lewis \& P. Mitchell, pp. 403-25. Erlbaum. [aJIMC, PM]

Chandler, M. J., Hallett, D. \& Sokol, B. (2001) Competing claims about competing knowledge claims. In: Personal epistemologies, ed. B. K. Hofer \& P. R. Pintrich. Erlbaum. [aJIMC]

Chandler, M. J. \& Lalonde, C. (1996) Shifting to an interpretive theory of mind: 5to 7-year-olds' changing conceptions of mental life. In: Reason and responsibility: The passage through childhood, ed. A. Sameroff \& M. Haith. University of Chicago Press. [aJIMC]

Chapman, M. (1986) The structure of exchange: Piaget's sociological theory. Human Development 29:181-94. [aJIMC]

(1987a) Inner processes and outward criteria: Wittgenstein's importance for psychology. In: Meaning and the growth of understanding: Wittgenstein's significance for developmental psychology, ed. M. Chapman \& R. A. Dixon, pp. 103-27. Springer-Verlag. [arJIMC]

(1987b) Piaget, attentional capacity, and the functional implications of formal structure. Advances in Child Development and Behavior 20:289-334. [arJIMC]

(1988a) Constructive evolution: Origins and development of Piaget's thought. Cambridge University Press. [aJIMC, SRL]

(1988b) Contextuality and directionality of cognitive development. Human Development 31:92-106. [aJIMC]

(1991) The epistemic triangle: Operative and communicative components of cognitive development. In: Criteria for competence: Controversies in the conceptualization and assessment of children's abilities, ed. M. Chandler \& M Chapman, pp. 209-28. Erlbaum. [JB, arJIMC, SH, OML]

(1992) Equilibration and the dialectics of organization. In: Piaget's theory: Prospects and possibilities, ed. H. Beilin \& P. B. Pufall. Erlbaum. [aJIMC] (1999) Constructivism and the problem of reality. Journal of Applied Development Psychology 20:31-43. [aJIMC, LS]

Chapman, M. \& Lindenberger, U. (1992) Transitivity judgments, memory for premises, and models of children's reasoning. Developmental Review 12:12463. [aJIMC]

Churchland, P. M. (1996) The engine of reason, the seat of the soul. MIT Press. [APC]

Clark, A. (1999) Where brain, body and world collide. Cognitive Systems Research 1(1):5-17. [APC]

Clements, W. A. \& Perner, J. (1994) Implicit understanding of belief. Cognitive Development 9:377-95. [aJIMC, TR]

Clements, W. A., Rustin, C. L. \& McCallum, S. (2000) Promoting the transition from implicit to explicit understanding: A training study of false belief. Developmental Science 3:81-92. [aJIMC]

Cole, K. \& Mitchell, P. (2000) Siblings in the development of executive control and a theory of mind. British Journal of Developmental Psychology 18:279-95. [aJIMC, TPG]

Cole, M. (1992) Context, modularity, and the cultural constitution of development. In: Children's development within social context, vol. 2, Research and methodology, ed. L. T. Winegar \& J. Valsiner. Erlbaum. [aJIMC]

Corkum, V. \& Moore, V. (1995) Development of joint visual attention in infants. In: Joint attention: Its origins and role in development, ed. C. Moore \& P. Dunham. Erlbaum. [aJIMC]

(1998) The origins of joint visual attention in infants. Developmental Psychology $34: 28-38$. [aJIMC]

Craig, A. P. (2003) Culture and the individual. Theory and Psychology 13:629-50. [APC]

Crick, N. R. \& Dodge, K. A. (1994) A review and reformulation of social information-processing mechanisms in children's social adjustment. Psychological Bulletin 115:74-101. [CEI]

Csibra, G., Biro, S., Koos, O. \& Gergely, G. (2003) One-year-old infants use teleological representations of actions productively. Cognitive Science 27:11133. $[\mathrm{CW}-\mathrm{B}]$

Currie, G. \& Ravenscroft, I. (2002) Recreative minds: Imagination in philosophy and psychology. Oxford University Press. [PM]

Cutting, A. L. \& Dunn, J. (1999) Theory of mind, emotion understanding, language, and family background: Individual differences and interrelations. Child Development 70:853-65. [aJIMC, JMJ]

Damasio, A. R. (2003) Looking for Spinoza: Joy, sorrow, and the feeling brain Harvest Books. $[\mathrm{PF}]$

Davidson, R. J., Putnam, K. M. \& Larson, C. L. (2000) Dysfunction in the neural circuitry of emotion regulation - a possible prelude to violence. Science 289(5479):591-94. [PF]

Deák, G. O., Ray, S. D. \& Brenneman, K. (2003) Children’s preservative appearance-reality errors are related to emerging language skills. Child Development 74:944-64. [MS]

Deneault, J., Morin, P. L., Ricard, M., Decarie, T. G. \& Quintal, G. (2003) Understanding of mind in twins: Does zygosity make a difference? Poster presented at the biennial meeting of the Society for Research in Child Development, Tampa, April 2003. [rJIMC]
Denham, S. A. (1998) Emotional development in young children. Guilford Press. [CEI]

Desrochers, S., Morissette, P. \& Ricard, M. (1995) Two perspectives on pointing in infancy. In: Joint attention: Its origins and role in development, ed. C. Moore \& P. J. Dunham. Erlbaum. [aJIMC, CW-B]

de Villiers, J. (2000) Language and theory of mind: What are the developmental relationships? In: Understanding of minds: Perspectives from developmental cognitive neuroscience, ed. S. Baron-Cohen, H. Tager-Flusberg \& D. J. Cohen. Oxford University Press. [aJIMC]

de Villiers J. G. \& de Villiers, P. A. (2000) Linguistic determinism and the understanding of false beliefs. In: Children's reasoning and the mind, ed. P. Mitchell \& K. J. Riggs, pp. 191-228. Psychology Press. [aJIMC, CAM]

de Villiers, J. G. \& Pyers, J. E. (2002) Complements to cognition: A longitudinal study of the relationship between complex syntax and false-belief understanding. Cognitive Development 17:1037-60. [JMJ]

Dewey, J. (1979) John Dewey: The middle works, 1899-1924 (vol. 7: 1912-1914). Southern Illinois University Press. [BWS]

Döbert, R. (2004) The development and overcoming of "universal pragmatics" in Piaget's thinking. In: Social interaction and the development of knowledge, ed. J. I. M. Carpendale \& U. Müller. Erlbaum. [aJIMC]

Dodge, K. A. \& Coie, J. D. (1987) Social-information-processing factors in reactive and proactive aggression in children's peer groups. Journal of Personality and Social Psychology 53:1146-58. [CEI]

Dunbar, R. (1996) Social intelligence and interaction: Expressions and implication of the social bias in human intelligence. Journal of the Royal Anthropological Institute 2(3):551-53. [OC]

Dunham, P., Dunham, F. \& O'Keefe, C. (2000) Two-year-olds' sensitivity to a parent's knowledge state: Mind reading or contextual cues? British Journal of Developmental Psychology 18:519-32. [CW-B]

Dunn, J. (1988) The beginning of social understanding. Harvard University Press. [aJIMC, TR]

(1996a) The Emmanuel Miller Memorial Lecture 1995. Children's relationships: Bridging the divide between cognitive and social development. Child Psychology and Psychiatry 37:507-18. [aJIMC, RB]

(1996b) Family conversations and the development of social understanding. In: Children, research and policy: Essays for Barbara Tizard, ed. B. Bernstein \& J. Brannen, pp. 81-95. Taylor \& Francis. [JWA]

(2002) Sibling relationships. In: Blackwell handbook of childhood social development, ed. P. K. Smith \& C. H. Hart, pp. 223-37. Blackwell. [NH]

Dunn, J., Brown, J. \& Beardsall, L. (1991a) Family talk about feeling states and children's later understanding of others' emotions. Developmental Psychology 27:448-55. [aJIMC, HS, ASW-A]

Dunn, J., Brown, J., Slomkowki, C., Tesla, C. \& Youngblade, L. (1991b) Young children's understanding of other people's feelings and beliefs: Individual differences and their antecedents. Child Development 62:1352-66. [ASW-A, aJIMC, EM, TR]

Duranti, A. (1997) Linguistic anthropology. Cambridge University Press. $[\mathrm{NB}]$

Duveen, G. (1997) Psychological development as a social process. In: Piaget, Vygotsky and beyond, ed. L. Smith, J. Dockrell \& P. Tomlinson. Routledge. [aJIMC]

Eckerman, C. O., Davis, C. C. \& Didow, S. M. (1989) Toddlers' emerging ways of achieving social coordination with a peer. Child Development 60:440-53. $[\mathrm{SZ}]$

Eddy, T. J., Gallup, G. G., Jr. \& Povinelli, D. J. (1993) Attribution of cognitive states to animals: Anthropomorphism in comparative perspective. Journal of Social Issues 49:87-101. [TJE]

Elias, N. (1978) What is sociology? Columbia Press. (Original work published in 1970.) [arJIMC]

El'konin, D. (1969) Some results of the study of the psychological development of preschool-age children. In: A handbook of contemporary Soviet psychology, ed. M. Cole \& I. Maltzman pp. 163-209. Basic Books. [OC]

Elman, J. L., Bates, E. A., Johnson, M. H., Karmiloff-Smith, A., Parisi, D. \& Plunkett, K. (1996) Rethinking innateness: A connectionist perspective on development. MIT Press. [VM]

Feldman, C. F. (1992) The new theory of theory of mind. Human Development 35:107-17. [aJIMC]

Fernyhough, C. (1996) The dialogic mind: A dialogic approach to the higher mental functions. New Ideas in Psychology 14:47-62. [JWA, arJIMC, $\mathrm{CF}]$

(1997) Vygotsky's sociocultural approach: Theoretical issues and implications for current research. In: The development of social cognition, ed. S. Hala, pp. 6593. Psychology Press. [CF]

Feuerstein, R. (1980) Instrumental enrichment. An intervention program for cognitive modifiability. University Park Press. [APC]

Field, T. (1985) Attachment as psychobiological attunement: Being on the same wavelength. In: The psychobiology of attachment and separation, ed. M. Reite $\&$ T. Fields, pp. 415-54. Academic Press. [PF] 
References/Carpendale \& Lewis: Constructing an understanding of mind

Figueras-Costa, B. \& Harris, P. L. (2001) Theory of mind development in deaf children: A nonverbal test of false belief understanding. Journal of Deaf Studies in Education 6:92-102. [aJIMC]

Fine, S. E., Izard, C. E., Mostow, A. J., Trentacosta, C. J. \& Ackerman, B. P. (2003) First grade emotion knowledge as a predictor of fifth grade self-reported internalizing behaviors in children from economically disadvantaged families. Development and Psychopathology 15:331-42. [CEI]

Fine, S. E., Trentacosta, C. J., Izard, C. E., Mostow, A. J. \& Campbell, J. L. (in press) Anger perception, caregivers' use of physical discipline, and aggression in children at risk. Social Development [CEI]

Flavell, J. H., Fry, C., Wright, J. \& Jarvis, P. (1968) The development of role-taking and communication skills in children. Wiley. [rJIMC]

Flavell, J. H. \& Miller, P. H. (1998) Social cognition. In: Handbook of child psychology, 5th edition, ed. W. Damon, D. Kuhn \& R. S. Siegler. Wiley. [aJIMC]

Fonagy, P., Gergely, G., Jurist, E. \& Target, M. (2002) Affect regulation, mentalization and the development of the self. Other Press. $[\mathrm{PF}]$

Fonagy, P., Redfern, S. \& Charman, T. (1997) The relationship between beliefdesire reasoning and a projective measure of attachment security (SAT). British Journal of Developmental Psychology 15:51-61. [aJIMC]

Fonagy, P., Steele, H., Moran, G., Steele, M. \& Higgitt, A. (1991a) The capacity for understanding mental states: The reflective self in parent and child and its significance for security of attachment. Infant Mental Health Journal 13:20017. $[\mathrm{PF}]$

Fonagy, P., Steele, H. \& Steele, M. (1991b) Maternal representations of attachment during pregnancy predict the organisation of infant-mother attachment at one year of age. Child Development 62:891-905. [EM]

Fonagy, P., Steele, H., Steele, M. \& Holder J. (1997) Attachment and theory of mind: Overlapping constructs? Association for Child Psychology and Psychiatry Occasional Papers 14:31-40. [PF,HS]

Fonagy, P. \& Target, M. (1997) Attachment and reflective function: Their role in self-organization. Development and Psychopathology 9:679-700. [aJIMC] (2002) Early intervention and the development of self-regulation. Psychoanalytic Inquiry 22(3):307-35. [PF]

Freeman, N. \& Lacohée, H. (1995) Making explicit 3-year-olds' implicit competence with their own false beliefs. Cognition 56:31-60. [SH]

Freeman, N. H., Lewis, C. \& Doherty, M. (1991) Preschoolers' grasp of a desire for knowledge in false-belief reasoning: Practical intelligence and verbal report. British Journal of Developmental Psychology 9:139-57. [aJIMC]

Frith, C. D. \& Frith, U. (1999) Interacting minds - A biological basis. Science 286:1692-95. [TPG]

Fritzley, V. H., \& Lee, K. (2003) Do young children always say yes to yes/no questions? A metadevelopmental study of the affirmation bias. Child Development 74:1297-313. [MS]

Frye, D., Zelazo, P. D. \& Palfai, T. (1995a) Inference and action in early causal reasoning. Cognitive Development 10:120-31. [aJIMC]

(1995b) Theory of mind and rule-based reasoning. Cognitive Development 10:483-527. [SH]

Gallagher, H. L. \& Frith, C. D. (2003) Functional imaging of "theory of mind." Trends in Cognitive Sciences 7:77-83. [TPG]

Garfield, J. L., Peterson, C. C. \& Perry, T. (2001) Social cognition, language acquisition and the development of the theory of mind. Mind and Language 16:494-541. [JWA, rJIMC, CF]

Garfinkel, H. (1967) Studies in ethnomethodology. Prentice-Hall. [JS]

Garnham, W. A. \& Perner, J. (2001) Actions really do speak louder than words but only implicitly: Young children's understanding of false belief in action. British Journal of Developmental Psychology 19:413-32. [TR]

Garnham, W. A. \& Ruffman, T. (2001) Doesn't see, doesn't know: Is anticipatory looking really related to understanding belief? Developmental Science 4:94100. [aJIMC,TR]

Garton, A. F. (2004) Exploring cognitive development. Blackwell. [OC]

Gauvain, M. (2001) The social context of cognitive development. The Guilford Press. [OC]

Gellatly, A. (1997) Why the young child has neither a theory of mind nor a theory of anything else. Human Development 40:32-50. [aJIMC]

Gergely, G. (2003) The development of teleological versus mentalizing observational learning strategies in infancy. Bulletin of the Menninger Clinic 67:113-31. [PF]

Gergely, G., Bekkering, H. \& Kiraly, I. (2002) Rational imitation in preverbal infants. Nature 415:755. [PF, CW-B]

Gergely, G. \& Csibra, G. (1997) Teleological reasoning in infancy: The infant's naive theory of rational action. A reply to Premack and Premack. Cognition 63:227-33. [PF]

(2003) Teleological reasoning in infancy: The naive theory of rational action. Trends in Cognitive Sciences 7:287-92. [PF]

Gergely, G., Nadasdy, Z., Csibra, G. \& Biro, S. (1995) Taking the intentional stance at 12 months of age. Cognition 56:165-93. [CW]
Gergely, G. \& Watson, J. S. (1996) The social biofeedback theory of parental affectmirroring: The development of emotional self-awareness and self-control in infancy: International Journal of Psychoanalysis 77:1181-212. [VM]

Gergen, K. J. (1994) Realities and relationships: Soundings of social construction. Harvard University Press. [aJIMC]

German, T. P. \& Leslie, A. M. (2000) Attending to and learning about mental states. In: Children's reasoning and the mind, ed. P. Mitchell \& K. J. Riggs, pp. 229-52. Psychology Press/Taylor \& Francis (UK). [aJIMC, TPG]

(2001) Children's inferences from "knowing" to "pretending" and "believing." British Journal of Developmental Psychology 19:59-83. [TPG]

Gerrans, P. (2002) The theory of mind module in evolutionary psychology. Biology and Philosophy 17:305-21. [rJIMC]

Gibbs, R. (2001) Intentions as emergent product of social interactions. In: Intention and intentionality: Foundation of social cognition, ed. F. Malle, L. Moses \& D. Baldwin, pp. 105-22. A Bradford Book/MIT Press. [OC]

Gillette, J., Gleitman, H., Gleitman, L. \& Lederer, A. (1999) Human simulations of vocabulary learning. Cognition 73(2):135-76. [CAM]

Gleitman, L. (1990) The structural sources of verb meanings. Language Acquisition 1(1):3-55. [CAM]

Glock, H.-J. (1996) Necessity and normativity. In: The Cambridge companion to Wittgenstein, ed. H. Sluga \& D. G. Stern, pp. 198-225. Cambridge University Press. [MHB]

Goldberg, A. (1995) Constructions: A construction grammar approach to argument structure. University of Chicago Press. [NB]

Goldberg, B. (1991) Mechanism and meaning. In: Investigating psychology: Sciences of the mind after Wittgenstein, ed. J. Hyman, pp. 48-66. Routledge. [arJIMC]

Göncï, A. (1993) Development of intersubjectivity in the dyadic play of preschoolers. Early Childhood Research Quarterly 8:99-116. [NH]

Gopnik, A. (1993) How we know our minds: The illusion of first-person knowledge of intentionality. Behavioral and Brain Sciences 16:1-14. [KB, aJIMC]

Gopnik, A. \& Meltzoff, A. (1998) Words, thoughts, and theories. MIT Press. [OML]

Gopnik, A., Slaughter, V. \& Meltzoff, A. (1994) Changing your views: How understanding visual perception can lead to a new theory of the mind. In: Children's early understanding of the mind, ed. C. Lewis \& P. Mitchell. Erlbaum. [aJIMC]

Gopnik, A. \& Wellman, H. M. (1992) Why the child's theory of mind really is a theory. Mind and Language 7:145-71. [KB, arJIMC, TR]

(1994) The theory theory. In: Mapping the mind: Domain specificity in cognition and culture, ed. L. A. Hirschfeld \& S. A. Gelman, pp. 257-93. Cambridge University Press. [KB, aJIMC]

Gordon, A. C. L. \& Olson, D. R. (1998) The relation between acquisition of a theory of mind and the capacity to hold in mind. Journal of Experimental Child Psychology 68:70-83. [aJIMC,CAM]

Gordon, R. M. (1992) The simulation theory: Objections and misconceptions. Mind and Language 7:11-34. [TR]

Gottlieb, G. (1991) Epigenetic systems view of human development. Developmental Psychology 27:33-34. [SH]

Greene, J. \& Haidt, J. (2002) How (and where) does moral judgment work? Trends in Cognitive Sciences 6(12):517-23. [PF]

Grice, H. (1975) Logic and conversation. In: Syntax and semantics III: Speech acts, ed. P. Cole \& J. Moran, pp. 41-58. Academic Press. [HS]

Habermas, J. (1983/1990) Moral consciousness and communicative action. MIT Press. (Original work published 1983.) [aJIMC, PF]

Hacker, P. M. S. (1990) Wittgenstein: Meaning and mind. Blackwell. [aJIMC] (1991) Seeing, representing and describing: An examination of David Marr's computational theory of vision. In: Investigating psychology: Sciences of the mind after Wittgenstein, ed. J. Hyman, pp. 119-54. Routledge. [arJIMC]

(1996) Wittgenstein's place in twentieth-century analytic philosophy. Blackwell. [aJIMC]

(1997) Wittgenstein: On human nature. Phoenix. [aJIMC]

Hacking, I. (1992) World-making by kind-making: Child-abuse for example. In: How classification works: Nelson Goodman among the social sciences, ed. M. Douglas \& D. Hull, pp. 180-238. Edinburgh University Press. [BWS]

Haden, C., Haine, R. \& Fivush, R. (1997) Developing narrative structure in parent-child conversations about the past. Developmental Psychology 33:295307. [ASW-A]

Hala, S. \& Carpendale, J. I. M. (1997) All in the mind: Children's understanding of mental life. In: The development of social cognition, ed. S. Hala. Psychology Press. [aJIMC]

Hala, S. \& Chandler, M. J. (1996) The role of strategic planning in accessing falsebelief understanding. Child Development 67:2948-66. [aJIMC]

Hala, S., Hug, S. \& Henderson, A. M. (2003) Executive function and false-belief understanding in preschool children: Two tasks are harder than one. Cognition and Development 4:275-98. [SH]

Hala, S. \& Russell, J. (2001) Executive control within strategic deception: A 
window on early cognitive development. Journal of Experimental Child Psychology 80:112-41. [SH]

Hanna, E. \& Meltzoff, A. N. (1993) Peer imitation by toddlers in laboratory, home and day-care contexts: Implications for social learning and memory. Developmental Psychology 29:701-10. [SZ]

Happé, F. G. E. (1994) An advanced test of theory of mind: Understanding of story characters' thoughts and feelings by able autistic, mentally handicapped, and normal children and adults. Journal of Autism and Developmental Disorders 24:129-54. [RB]

(1995) The role of age and verbal ability in the theory of mind task performance of subjects with autism. Child Development 66:843-55. [JWA,aJIMC]

Harris, P. L (1991) The work of imagination. In: Natural theories of mind, ed. A. Whiten, pp. 283-304. Blackwell. [aJIMC, OML]

(1994a) Thinking by children and scientists: False analogies and neglected similarities. In: Mapping the mind: Domain specificity in cognition and culture, ed. L. A. Hirschfeld \& S. A. Gelman. Cambridge University Press. [aJIMC]

(1994b) Understanding pretense. In: Origins of an understanding of mind, ed. C. Lewis \& P. Mitchell, pp. 235-59. Erlbaum. [ASW-A]

(1996) Desires, beliefs, and language. In: Theories of theories of mind, ed. P. Carruthers \& P. K. Smith. Cambridge University Press. [aJIMC]

(1999) Acquiring the art of conversation. In: Developmental psychology: Achievements and prospects, ed. M. Bennett, pp. 89-105. Psychology Press/ Taylor \& Francis. [JWA]

(2000) The work of the imagination. Blackwell. [aJIMC]

(in press) Conversation, pretence, and theory of mind. In: Why language matters for theory of mind, ed. J. W. Astington \& J. Baird. Oxford University Press. [rJIMC]

Haviland, J. J. \& Lelwica, M. (1987) The induced affect response: 10-week-old infants' responses to three emotion expressions. Developmental Psychology 23:97-104. [CEI]

Hay, D. \& Ross, H. (1982) The social nature of early conflict. Child Development 53:105-13. [SZ]

Heal, J. (1996) Simulation, theory and content. In: Theories of theories of mind, ed. P. Carruthers \& P. K. Smith. Cambridge University Press. [PM]

Hegel, G. (1807) The phenomenology of spirit. Oxford University Press. [PF]

Heil, J. (1981) Does cognitive psychology rest on a mistake? Mind 90:321-42. [rJIMC]

Hejmadi, A., Rozin, P. \& Siegal, M. (in press) Once in contact, always in contact: Conceptions of essence and purification in Hindu Indian and American children. Developmental Psychology. [MS]

Herrera, C. \& Dunn, J. (1997) Early experiences with family conflict: Implications for arguments with a close friend. Developmental Psychology 33:869-88. $[\mathrm{JMJ}]$

Higgins, E. T. \& Parsons, J. E. (1985) Social cognition and the social life of the child: Stages as subcultures. In: Social cognition and social development: A sociocultural perspective, ed. E. T. Higgins, D. N. Ruble \& W. W. Hartup. Cambridge University Press. [RB]

Hinde, R. (1979) Towards understanding relationships. Academic Press. [NH]

Hirsh-Pasek, K. \& Golinkoff, R. M. (1996) The origins of grammar: Evidence from early language comprehension. MIT Press. [DEM]

Hobson, R. P. (1991) Against the theory of "theory of mind." British Journal of Developmental Psychology 9:33-51. [aJIMC]

(1993) Autism and the development of mind. Erlbaum. [aJIMC]

(1994) Perceiving attitudes, conceiving minds. In: Children's early understanding of mind: Origins and development, ed. C. Lewis \& P. Mitchell, pp. 71-93. Erlbaum. [arJIMC]

(2002) The cradle of thought: Explorations of the origins of thinking. Macmillan. [arJIMC, CEI]

(in press) What puts the jointness into joint attention? In: Joint attention: Communication and other minds, ed. N. Eilan, C. Hoerl, T. McCormak \& J. Roessler. Oxford University Press. [rJIMC]

Hoff-Ginsberg, E. (1986) Function and structure in maternal speech: Their relation to the child's development of syntax. Developmental Psychology 22:155-63. [TR]

Hoff-Ginsberg, E. \& Shatz, M. (1982) Linguistic input and the child's acquisition of language. Psychological Bulletin 92:3-26. [TR]

Holmes, H. A., Black, C. \& Miller, S. A. (1996) A cross-task comparison of falsebelief understanding in a Head Start population. Journal of Experimental Child Psychology 63:263-85. [aJIMC]

Holquist, M. (1990) Dialogism: Bakhtin and his world. Routledge. [CF]

Hooven, C., Gottman, J. M. \& Katz, L. F. (1995) Parental meta-emotion structure predicts family and child outcomes. Cognition and Emotion 9:229-64. [aJIMC]

Horgan, J. (1999) The undiscovered mind. Free Press. [OML]

Howe, N., Rinaldi, C., Jennings, M. \& Petrakos, H. (2002) "No! the lambs can stay out because they got cosies": Constructive and destructive sibling conflict, pretend play, and social understanding. Child Development 73:1460-73. $[\mathrm{NH}]$

Howes, C. \& Stewart, P. (1987) Child's play with adults, toys, and peers: An examination of family and child care influences. Developmental Psychology 23:423-30. [SZ]

Howes, C., Unger, O. \& Seidner, L. B. (1989) Social pretend play in toddlers: Parallels with social play and with solitary pretend. Child Development 60:7784. [SZ]

Hubbard, J. A. (2001) Emotion expression processes in children's peer interaction: The role of peer rejection, aggression, and gender. Child Development 72:1426-38. [CEI]

Hudson, J. A. (1990) The emergence of autobiographic memory in mother-child conversation. In: Knowing and remembering in young children, ed. R. Fivush \& J. A. Hudson, pp. 166-96. Cambridge University Press. [ASW-A]

(1993) Understanding events: The development of script knowledge. In: The Child as psychologist: An introduction to the development of social cognition, ed. M. Bennett, pp. 142-67. Simon \& Schuster. [ASW-A]

Hudson, J. A., Shapiro, L. R. \& Sosa, B. B. (1995) Planning in the real world: Preschool children's scripts and plans for familiar events. Child Development 66:984-98. [ASW-A]

Hughes, C. (1998) Executive function in preschoolers: Links with theory of mind and verbal ability. British Journal of Developmental Psychology 16:233-53. [SH]

Hughes, C. \& Cutting, A. L. (1999) Nature, nurture, and individual differences in early understanding of mind. Psychological Science 10:429-32. [rJIMC, TPG]

Hughes, C., Deater-Deckard, K. \& Cutting, A. L. (1999) "Speak roughly to your little boy?" Sex differences in the relations between parenting and preschoolers' understanding of mind. Social Development 8:143-60. [aJIMC]

Hughes, C., Happé, F., Jaffee, S., Caspi, A. \& Moffitt, T. (2002) Theory of mind and verbal ability in a large sample of 5 -year-old twins: Evidence for genetic modularity? Paper presented at the International Conference "Why Language Matters for Theory of Mind," Toronto, Canada, April 2002. [JWA]

Hughes, C., Jaffee, S., Happé, F., Jackson, J., Taylor, A. \& Moffitt, T. E. (in press) Origins of individual differences in theory of mind and verbal ability at age five: Nature, nurture and their interplay. Child Development. [rJIMC]

Hutchins, E. (1987) Learning to navigate in context. Paper presented at the Workshop on Context, Cognition, and Activity, Stenugsund, Sweden, 1987. [JMJ]

(1995) Cognition in the wild. MIT Press. [APC]

Izard, C. E. (1978) On the ontogenesis of emotions and emotion-cognition relationships in infancy. In: The development of affect, ed. M. Lewis \& L. A. Rosenblum, pp. 389-413. Plenum Press. [CEI]

(2001) Emotional intelligence or adaptive emotions? Emotion 1:249-57. [CEI]

(2002) Translating emotion theory and research into preventive interventions. Psychological Bulletin 128:796-824. [CEI]

Izard, C. E., Fantauzzo, C. A., Castle, J. M., Haynes, O. M., Rayias, M. F. \& Putnam, P. H. (1995) The ontogeny and significance of infants' facial expressions in the first 9 months of life. Developmental Psychology 31:9971013. [CEI]

Izard, C. E., Fine, S. E., Schultz, D., Mostow, A. J., Ackerman, B. P. \& Youngstrom, E. A. (2001) Emotion knowledge as a predictor of social behavior and academic competence in children at risk. Psychological Science 12:18-23. [CEI]

Jenkins, J. M. \& Astington, J. W. (1996) Cognitive factors and family structure associated with theory of mind development in young children. Developmental Psychology 32:70-78. [JWA, aJIMC, JMJ]

(2000) Theory of mind and social behavior: Causal models tested in a longitudinal study. Merrill Palmer Quarterly 46:203-20. [ JMJ]

Jenkins, J. M., Turrell, S., Kogushi, Y., Lollis, S. \& Ross, H. A. (2003) Longitudinal investigation of the dynamics of mental state talk in families. Child Development 74:905-20. [JMJ]

Johnson, M. H. \& Morton, J. (1991) Biology and cognitive development. Blackwell. [TR]

Johnson, S. C. (2003) Detecting agents. Philosophical Transactions of the Royal Society of London, Series B 358:549-59. [TPG]

Jopling, D. (1993) Cognitive science, other minds, and the philosophy of dialogue. In: The perceived self, ed. U. Neisser. MIT Press. [aJIMC]

Kahana-Kalman, R. \& Walker-Andrews, A. S. (2001) The role of person familiarity in young infants' perception of emotional expressions. Child Development 72:352-69. [ASW-A]

Kamii, C. (1982) Encouraging thinking in mathematics. Phi Delta Kappan 64:24751. $[\mathrm{LS}]$

Karmiloff-Smith, A. (1998) Development itself is the key to understanding developmental disorders. Trends in Cognitive Science 2:389-98. [VM] 
References/Carpendale \& Lewis: Constructing an understanding of mind

Kaye, K. (1982) The mental and social life of babies. Harvester Wheatsheaf. [arJIMC]

Kelley, W. M., Macrae, C. N., Wyland, C. L., Caglar, S., Inati, S. \& Heatherton, T. F. (2002) Finding the self? An event-related fMRI study. Journal of Cognitive Neuroscience 14(5):785-94. [PF]

Kenny, A. (1991) The homunculus fallacy. In: Investigating psychology: Sciences of the mind after Wittgenstein, ed. J. Hyman, pp. 155-65. Routledge. [rJIMC]

Kitchener, R. F. (1986) Piaget's theory of knowledge. Yale University Press. [aJIMC]

(2004) Piaget's social epistemology. In: Social interaction and the development of knowledge, ed. J. I. M. Carpendale \& U. Müller. Erlbaum. [rJIMC]

Kochanska, G. (2001) Emotional development in children with different attachment histories: The first three years. Child Development 72:474-90. $[\mathrm{PF}]$

Kochanska, G., Murray, K. \& Harlan, E. (2000) Effortful control in early childhood: Continuity and change, antecedents, and implications for social development. Developmental Psychology 36:220-32. [PF]

Koren-Karie, N., Oppenheim, D., Dolev, S., Sher, S. \& Etzion-Carasso, A. (2002) Mother's insightfulness regarding their infants' internal experience: Relations with maternal sensitivity and infant attachment. Developmental Psychology 38:534-42. [PF]

Koulomzin, M., Beebe, B., Anderson, S., Jaffe, J., Feldstein, S. \& Crown, C. (2002) Infant gaze, head, face, and self-touch at 4 months differentiate secure vs. avoidant attachment at one year: A microanalytic approach. Attachment and Human Development 4:3-24. [HS]

Kraemer, G. W. (1999) Psychobiology of early social attachment in Rhesus monkeys: Clinical applications. In: The integrative neurobiology of affiliation, ed. C. S. Carter, I. I. Lederhendler \& B. Kirkpatrick, pp. 373-90. MIT Press. $[\mathrm{PF}]$

Kreppner, J. M., O’Connor, T. G. \& Rutter, M. (2001) Can inattention/overactivity be an institutional deprivation syndrome? Journal of Abnormal Child Psychology 29(6):513-28. [PF]

Kripke, S. A. (1982) Wittgenstein on rules and private language. Harvard University Press. [aJIMC]

Kuebli, J., Butler, S. \& Fivush, R. (1995) Mother-child talk about past emotions: Relations of maternal language and child gender over time. Cognition and Emotion 9:265-83. [aJIMC]

Kusch, M. (1997) The sociophilosophy of folk psychology. Studies in History and Philosophy of Science 28:1-25. [BWS]

(1999) Psychological knowledge: A social history and philosophy. Routledge. [BWS]

Lagattuta, K. H. \& Wellman, H. M. (2002) Differences in early parent-child conversations about negative versus positive emotions: Implications for the development of psychological understanding. Developmental Psychology 38:564-80. [RAT]

Laible, D. J. \& Thompson, R. A. (2002) Mother-child conflict in the toddler years: Lessons in emotion, morality, and relationships. Child Development 73:1187203. [rJIMC, RAT]

Lakatos, I. (1970) Falsification and the methodology of scientific research programmes. In: Criticism and the growth of knowledge, ed. I. Lakatos \& A. Musgrave, pp. 91-196. Cambridge University Press. [rJIMC]

Lalonde, C. E. \& Chandler, M. J. (2002) Children's understanding of interpretation. New Ideas in Psychology 20:163-98. [aJIMC]

Landau, B. \& Gleitman, L. (1985) Language and experience: Evidence from the blind child. Harvard University Press. [CAM]

Landry, S. H., Miller-Loncar, C. L. Smith, K. E. \& Swank, P. R. (2002) The role of early parenting in children's development of executive processes. Developmental Neuropsychology 21:15-41. [rJIMC]

Lawrence, J. A. \& Valsiner, J. (1993) Conceptual roots of internalization: From transmission to transformation. Human Development 36:150-67. [aJIMC]

Lee, K., Cameron, C. A., Xu, F., Fu, G. \& Broad, J. (1997) Chinese and Canadian children's evaluations of lying and truth-telling. Child Development 64:92434. [MS]

Leekam, S. (in press) Why do children with autism have a joint attention impairment? In: Joint attention: Communication and other minds, ed. N. Eilan, C. Hoerl, T. McCormak \& J. Roessler. Oxford University Press. $[\mathrm{rJIMC}]$

Leekam S. \& Ramsden, C. (2003) Dyadic orienting and the problem of interpersonal engagement. Paper presented at the Society for Research in Child Development, Tampa, FL, April 2003. [SRL]

Leslie, A. M. (1987) Pretense and representation: The origins of "theory of mind." Psychological Review 94:412-26. [TPG]

(1994) Pretending and believing: Issues in the theory of ToMM. Cognition 50:211-38. [TPG]

(2000) "Theory of mind" as a mechanism of selective attention. In: The new cognitive neurosciences, 2nd edition, ed. M. Gazzaniga, pp. 1235-47. MIT Press. [TPG]
Leslie, A. M. \& Polizzi, P. (1998) Inhibitory processing in the false belief task: Two conjectures. Developmental Science 1:247-53. [TPG, SH]

Levine, L. J., Stein, N. L. \& Liwag, M. D. (1999) Remembering children's emotions: Sources of concordant and discordant accounts between parents and children. Developmental Psychology 35:790-801. [RAT]

Lewis, C. \& Carpendale, J. I. M. (2002) Social cognition. In: The handbook of social development, ed. P. K. Smith \& C. Hart. Blackwell. [aJIMC]

Lewis, C., Freeman, N. H., Kyriakidou, C., Maridaki-Kassotaki, K. \& Berridge, D M. (1996) Social influences on false belief access: Specific sibling influences or general apprenticeship? Child Development 67:2930-47. [aJIMC]

Lewis, C. \& Mitchell, P., eds. (1994) Children's early understanding of mind: Origins and development. Erlbaum. [aJIMC]

Lillard, A. S. (1993a) Pretend play skills and the child's theory of mind. Child Development 64:348-71. [aJIMC]

(1993b) Young children's conceptualization of prentense: Action or mental representational state? Child Development 64:372-86. [aJIMC]

(1998) Ethnopsychologies: Cultural variations in theories of mind. Psychological Bulletin 123:3-32. [aJIMC, PGV, CW-B]

(2002) Pretend play and cognitive development. In: Blackwell handbook of childhood cognitive development, ed. U. Goswami, pp. 206-26. Blackwell. [NH]

Lloyd, P. \& Fernyhough, C., eds. (1999) Lev Vygotsky: Critical assessments (4 volumes). Routledge. [DKS]

Lock, A., ed. (1978) Action, gesture and symbol: The emergence of language. Academic Press. [aJIMC]

(1980) The guided reinvention of language. Academic Press. [aJIMC]

Loewenstein, G. F., Weber, E. U., Hsee, C. K. \& Welch, N. (2001) Risk as feelings. Psychological Bulletin 127:267-86. [CEI]

Lohmann, H. \& Tomasello, M. (2003) The role of language in the development of false belief understanding: A training study. Child Development 74:1130-44. [JWA, rJIMC]

Lourenço, O. (2001) The danger of words: A Wittgensteinian lesson for developmentalists. New Ideas in Psychology 19:89-115. [OML]

Lourenço, O. \& Machado, A. (1996) In defense of Piaget’s theory: A reply to 10 common criticisms. Psychological Review 103:143-64. [arJIMC]

Lundy, B. L. (2003) Father- and mother-infant face-to-face interactions: Differences in mind-related comments and infant attachment? Infant Behavior and Development 26:200-12. [EM]

Luria, A. (1969) Speech development and the formation of mental processes. In: A handbook of contemporary Soviet psychology, ed. M. Cole \& I. Maltzman, pp. 121-69. Basic Books. [OC]

Main, M. (2000) The organized categories of infant, child and adult attachment: Flexible vs. inflexible attention under attachment-related stress. Journal of the American Psychoanalytic Association 48:1055-96. [PF]

Main, M., Kaplan, N. \& Cassidy, J. (1985) Security in infancy, childhood, and adulthood: A move to the level of representation. In: Growing points of attachment theory and research, ed. I. Bretherton \& E. Waters. Monographs of the Society for Research in Child Development 50:66-104. [HS]

Malatesta, C. Z., Culver, C., Tesman, J. C. \& Shepard, B. (1989) The development of emotion expression during the first two years of life: Normative trends and patterns of individual differences. Monographs of the Society for Research in Child Development 54. [CEI]

Malcolm, N. (1991) The relation of language to instinctive behaviour. In: Investigating psychology: Sciences of the mind after Wittgenstein, ed. J. Hyman. Routledge. [aJIMC]

Maridaki-Kassotaki, K., Lewis, C. \& Freeman, N. H (2003) Lexical choice can lead to problems: What false-belief tests tell us about Greek alternative verbs of agency. Journal of Child Language 30:145-64. [aJIMC]

Matusov, E. (1998) When solo activity is not privileged: Participation and internalization models of development. Human Development 41:326-49. [aJIMC]

Mayes, L. C. (2000) A developmental perspective on the regulation of arousal states. Seminars in Perinatology 24:267-79. [PF]

McDowell, J. (1984) Wittgenstein on following a rule. Synthese 58:325-63. [aJIMC]

McGeer, V. (2001) Psycho-practice, psycho-theory and the contrastive case of autism: Journal of Consciousness Studies 8:109-32. [VM]

McGeer, V. \& Pettit, P. (2002) The self-regulating mind. Language and Communication 22:281-99. [VM]

Meehl, P. (1978) Theoretical risks and tabular asterisks: Sir Karl, Sir Ronald, and the slow progress of soft psychology. Journal of Consulting and Clinical Psychological 46:806-34. [OML]

Meerum Terwogt, M. \& Stegge, H. (1996) Children's perspective on the emotional process. In: The social child, ed. A. Campbell \& S. Muncer. Psychology Press. $[\mathrm{RB}]$

Meins, E. (1997) Security of attachment and the social development of cognition. Psychology Press. [aJIMC] 
(1999) Sensitivity, security and internal working models: Bridging the transmission gap. Attachment and Human Development 1:325-42. [aJIMC, RAT]

Meins, E. \& Fernyhough, C. (1999) Linguistic acquisitional style and mentalising development: The role of maternal mind-mindedness. Cognitive Development 14:363-80. [aJIMC]

Meins, E., Fernyhough, C., Fradley, E. \& Tuckey, M. (2001) Rethinking maternal sensitivity: Mothers' comments on infants' mental processes predict security of attachment at 12 months. Journal of Child Psychology and Psychiatry 42:637-48. [aJIMC, PF, EM]

Meins, E., Fernyhough, C., Russell, J. \& Clark-Carter, D. (1998) Security of attachment as a predictor of symbolic and mentalising abilities: A longitudinal study. Social Development 7:1-24. [aJIMC]

Meins, E., Fernyhough, C., Wainwright, R., Clark-Carter, D., Das Gupta, M. Fradley, E. \& Tuckey, M. (2003) Pathways to understanding mind: Construct validity and predictive validity of maternal mind-mindedness. Child Development 74:1194-211. [CF]

Meins, E., Fernyhough, C., Wainwright, R., Das Gupta, M., Fradley, E. \& Tuckey, M. (2002) Maternal mind-mindedness and attachment security as predictors of theory of mind understanding. Child Development 73:1715-26. [aJIMC, CF, EM, DKS]

Meltzoff, A. N. (1990) Foundations for developing a concept of self: The role of imitation in relating self to other and the value of social mirroring, social modelling and self practice in infancy. In: The self in transition: Infancy to childhood, ed. D. Cicchetti \& M. Beeghly. University of Chicago Press. [VM]

(1995) Understanding the intentions of others: Re-enactment of intended acts by 18-month-old children. Developmental Psychology 31:838-50. [CW-B]

Meltzoff, A. N. \& Gopnik, A. (1993) The role of imitation in understanding persons and developing a theory of mind. In: Understanding other minds: Perspectives from autism, ed. S. Baron-Cohen, H. Tager-Flusberg \& D. J. Cohen, pp. 33566. Oxford University Press. [VM]

Meltzoff, A. N., Gopnik, A. \& Repacholi, B. M. (1999) Toddlers' understanding of intentions, desires, and emotions: Explorations of the dark ages. In: Developing theories of intention, ed. P. D. Zelazo, J. W. Astington \& D. R. Olson. Erlbaum. [aJIMC]

Meltzoff, A. N. \& Moore, M. K. (1992) Early imitation within a functional framework: The importance of person identity, movement and development. Infant Behavior and Development 15:479-505. [VM]

Merleau-Ponty, M. (1964) The child's relations with others. In: The primacy of perception, ed. M. Merleau-Ponty. Northwestern Press. (Original work published 1960). [aJIMC]

Millikan, R. G. (1993) White queen psychology and other essays for Alice. MIT Press. [rJIMC]

(1995) White queen psychology and other essays. MIT Press. [APC]

Minter, M., Hobson, R. P. \& Bishop, M. (1998) Congenital visual impairment and "theory of mind." British Journal of Developmental Psychology 16:183-96. [aJIMC]

Mitchell, P. \& Riggs, K. J., eds. (2000) Children's reasoning and the mind. Psychology Press. [aJIMC]

Miyake, A., Friedman, N. P., Emerson, M. J., Witzki, A. H., Howerter, A. \& Wager, T. D. (2000) The unity and diversity of executive functions and their contributions to complex "frontal lobe" tasks: A latent variable analysis. Cognitive Psychology 41:49-100. [rJIMC]

Moissinac, L. \& Budwig, N. (2000) The development of desire terms in early child German. Psychology of Language and Communication 4(1):5-25. [NB]

Monsell, S. (1996) Control of mental processes. In: Unsolved mysteries of the mind: Tutorial essays in cognition, ed. V. Bruce. Erlbaum. [rJIMC]

Montague, D. P. \& Walker-Andrews, A. S. (2002) Mothers, fathers, and infants: The role of person familiarity and parental involvement in infants' perception of emotion expressions. Child Development 73:1339-52. [ASW-A]

Montgomery, D. E. (1997) Wittgenstein's private language argument and children's understanding of mind. Developmental Review 17:291-320. [arJIMC]

(2002) Mental verbs and semantic development. Journal of Cognition and Development 3:357-84. [JWA, rJIMC, DEM]

Moore, C. (1996) Evolution and the modularity of mindreading. Cognitive Development 11:605-21. [aJIMC]

(1999) Intentional relations and triadic relations. In: Developing theories of intention, ed. P. D. Zelazo, J. W. Astington, \& D. R. Olson. Erlbaum. [aJIMC, CW-B]

Moore, C. \& Corkum, V. (1994) Social understanding at the end of the first year of life. Developmental Review 14:349-72. [arJIMC]

Moore, C. \& D’Entremont, B. (2001) Developmental changes in pointing as a function of parent's attentional focus. Journal of Cognition and Development 2:109-29. [aJIMC, CW-B]

Moore, C., Furrow, D., Chiasson, L. \& Patriquin, M. (1994) Developmental relationships between production and comprehension of mental terms. First Language 14:1-17. [aJIMC]

Morton, J. (1989) The origins of autism. New Scientist 1694:44-7. [PF]

Moses, L. J. (2001) Executive accounts of theory-of-mind development. Child Development 72:688-90. [aJIMC, SH]

Müller, U. (1999) Wittgenstein and Piaget: A critique of mechanistic theories of meaning. Paper presented at the annual meeting of the Jean Piaget Society, Mexico City. June. [rJIMC]

Müller, U. \& Carpendale, J. I. M. (2001) Objectivity, intentionality, and levels of explanation. Behavioral and Brain Sciences 24:55-56. [ar]IMC]

(2004) From joint activity to joint attention: A relational approach to social development in infancy. In: Social interaction and the development of knowledge, ed. J. I. M. Carpendale \& U. Müller. Erlbaum. [rJIMC]

Müller, U. \& Runions. K. (2003) The origins of understanding of self and other: James Mark Baldwin's theory. Developmental Review 23:29-54. [aJIMC]

Müller, U., Sokol, B. \& Overton, W. F. (1998a) Constructivism and development: Reply to Smith's commentary. Developmental Review 18:228-36. [rJIMC]

(1998b) Reframing a constructivist model of the development of mental representations: The role of higher-order operations. Developmental Review 18:155-201. [rJIMC]

Murphy, C. M. \& Messer, D. J. (1977) Mothers, infants and pointing: A study of gesture. In: Studies in mother-infant interaction, ed. H. R. Schaffer. Academic Press. [aJIMC]

Muzik, M. \& Rosenblum, K. L. (2003) Maternal reflective capacity: Associations with sensitivity and mental state comments during interaction. Paper presented at the Biennial Meeting of the Society for Research in Child Development, Tampa, Florida, April 2003. [PF]

Nelson, K. (1985) Making sense: The acquisition of shared meaning. Academic Press. [rJIMC, KN]

(1993) The psychological and social origins of autobiographical memory. Psychological Science 4:1-8. [ASW-A]

(1996) Language in cognitive development: The emergence of the mediated mind. Cambridge University Press. [JWA, rJIMC, KN, RAT]

(1997) Cognitive change as collaborative construction. In: Change and development: Issues of theory, method, and application, ed. E. Amsel \& K. A Renninger, pp. 99-117. Erlbaum. [aJIMC, KN]

(1999) Event representations, narrative development and internal working models. Attachment and Human Development 1:239-52. [HS]

(in press) Language pathways to the community of minds. In: Why language matters to theory of mind, ed. J. W. Astington \& J. Baird. [KN]

Nelson, K., ed. (1986) Event knowledge: Structure and function in development. Erlbaum. [ASW-A]

Nelson, K., Henseler, S. \& Plesa, D. (2000) Entering a community of minds: A feminist perspective on theory of mind development. In: Toward a feminist developmental psychology, ed. P. Miller \& E. S. Scholnick, pp. 61-84. Routledge. [KN]

Nelson, K., Plesa Skwerer, D., Goldman, S., Henseler, S., Presler, N. \& Walkenfeld, F. F. (2003) Entering a community of minds: An experiential approach to "theory of mind." Human Development 46:24-46. [aJIMC, KN]

Nelson, K., Plesa, D. \& Henselser, S. (1998) Children's theory of mind: An experiential interpretation. Human Development 41:7-29. [aJIMC, KN]

Nelson, K. \& Shaw, L. K. (2002) Developing a socially shared symbolic system. In: Language, literacy and cognitive development, ed. E. Amsel \& J. Byrnes, pp. 27-58. Erlbaum. [KN]

Nelson, K., Skwerer, D., Goldman, S., Presler, N. \& Walkenfeld, F. (2003) Entering a community of minds: An experiential approach to "theory of mind." Human Development 46:24-46. [OML]

Newton, P., Reddy, V. \& Bull, R. (2000) Children's everyday deception and performance on false-belief tasks. British Journal of Developmental Psychology 18:297-317. [aJIMC]

Oatley, K. (2003) Fiction's sources: Conversation and imagination. Fiction’s principal accomplishment: The idea of character. Paper presented at the Conference on Narrative: Art and Mind, Cumberland Lodge, Windsor Great Park, May 30-June 2, 2003. [ JMJ]

Oatley, K. \& Larocque, L. (1995) Everyday concepts of emotions following everyother-day errors in joint plans. In: Everyday conceptions of emotions: An introduction to the psychology, anthropology, and linguistics of emotion, ed. J. Russell, J.-M. Fernandez-Dols, A. S. R. Manstead \& J. Wellenkamp, pp 14565. NATO ASI Series D 81. Kluwer. [JMJ]

Ochs, E. (1996) Linguistic resources for socializing humanity. In: Rethinking linguistic relativity, ed. J. Gumperz \& S. Levinson, pp. 407-37. Cambridge University Press. [NB]

Oppenheim, D. \& Koren-Karie, N. (2002) Mothers' insightfulness regarding their children's internal worlds: The capacity underlying secure child-mother relationships. Infant Mental Health Journal 23:593-605. [PF]

Overton, W. F. (1994) Contexts of meaning: The computational and the embodied 
mind. In: The nature and ontogenesis of meaning, ed. W. F. Overton \& D. S. Palermo. Erlbaum. [aJIMC]

(1998a) Developmental psychology: Philosophy, concepts, and methodology. In: Handbook of child psychology, vol. 1, 5th edition: Theoretical models of human development, ed. R. M. Lerner (W. Damon, Ed.-in-Chief), pp. 10788. Wiley. [rJIMC]

(1998b) Relational-developmental theory: A psychological perspective. In: Children, cities, and psychological theories, ed. D. Görlitz, H. J. Harloff, G. Mey \& J. Valsiner. Walter de Gruyter. [aJIMC]

Panksepp, J., Nelson, E. \& Bekkedal, M. (1999) Brain systems for the mediation of social separation-distress and social-reward: Evolutionary antecedents and neuropeptide intermediaries. In: The integrative neurobiology of affiliation, ed. C. S. Carter, I. I. Lederhendler \& B. Kirkpatrick, pp. 221-43. MIT Press. $[\mathrm{PF}]$

Parker, J. G. \& Gottman, J. M. (1989) Social and emotional development in a relational context. In: Peer relationships in child development, ed. J. T. Berndt $\&$ G. W. Ladd. Wiley. [RB]

Perner, J. (1988) Developing semantics for theories of mind: From propositional attitudes to mental representation. In: Developing theories of mind, ed. J. W. Astington, P. L. Harris \& D. R. Olson, pp. 141-72. Cambridge University Press. [PM]

(1991) Understanding the representational mind. MIT Press. [aJIMC, CAM, TR]

Perner, J. \& Astington, J. W. (1992) The child's understanding of representation. In: Piaget's theory: Prospects and possibilities, ed. H. Beilin \& P. B. Pufall, pp. 141-60. Erlbaum. [rJIMC]

Perner, J., Baker, S. \& Hutton, D. (1994) Prelief: The conceptual origins of belief and pretence. In: Children's early understanding of mind: Origins and development, ed. C. Lewis \& P. Mitchell, pp. 261-86. Erlbaum. [aJIMC, $\mathrm{TR}]$

Perner, J., Ruffman, T. \& Leekam, S. R. (1994) Theory of mind is contagious: You catch it from your sibs. Child Development 65:1228-38. [aJIMC, SZ]

Perner, J., Stummer, S., Sprung, M. \& Doherty, M. (2002) Theory of mind finds its Piagetian perspective: Why alternative naming comes with understanding belief. Cognitive Development 17:1451-72. [CAM]

Perner, J. \& Wimmer, H. (1985) "John thinks that Mary thinks that ...” Attribution of second-order beliefs by 5 - to 10 year old children. Journal of Experimental Child Psychology 39:437-71. [OC]

Peskin, J. \& Ardino, V. (2003) Representing the mental world in children's social behavior: Playing hide-and-seek and keeping a secret. Social Development. 12:496-512. [aJIMC, SRL]

Peterson, C. C., Peterson, J. L. \& Webb, J. (2000) Factors influencing the development of a theory of mind in blind children. British Journal of Developmental Psychology 18:431-47. [aJIMC]

Peterson, C. C. \& Siegal, M. (2000) Insights into theory of mind from deafness and autism. Mind and Language 15:123-45. [JWA, aJIMC]

(2002) Mindreading and moral awareness in popular and rejected preschoolers. British Journal of Developmental Psychology 20:205-24. [MS]

Phillips, A. T., Wellman, H. M. \& Spelke, E. S. (2002) Infants' ability to connect gaze and emotional expression to intentional action. Cognition 85:53-78. [CW-B]

Piaget, J. (1924/1928) Judgment and reasoning in the child. Routledge. (Original work published 1924.) [aJIMC]

(1945/1962) Play, dreams and imitation in childhood. Norton. (Original work published 1945.) [aJIMC, PF]

(1936/1963) The origins of intelligence in children. Norton. (Original work published 1936.) [aJIMC]

(1965a) Discussion. In: Entretiens sur les notions de genèse et de structure, ed. M. de Gandillac, L. Goldmann \& J. Piaget. Mouton. [LS]

(1932/1965b) The moral judgment of the child, trans. M. Gabian. Free Press. (Original work published 1932.) [arJIMC, SZ]

(1967) Six psychological studies. Vintage Books/Random House. [rJIMC, CAM]

(1937/1971) The construction of reality in the child. Ballantine. (Original work published 1937.) [aJIMC]

(1977/1995) Sociological studies. Routledge. (Original work published 1977.) [arJIMC, LS]

(1962/2000) Commentary on Vygotsky's criticism of language and thought of the child and judgement and reasoning in the child. New Ideas in Psychology 18:241-59. (Original work published 1962.) [rJIMC]

Piaget, J. \& Inhelder, B. (1948/1967) The child's conception of space. Norton. (Original work published 1948.) [aJIMC]

Pollak, S. D. \& Sinha, P. (2002) Effects of early experience on children's recognition of facial displays of emotion. Developmental Psychology 38:78491. [HS]

Povinelli, D. J. (1999) Social understanding in chimpanzees: New evidence from a longitudinal approach. In: Developing theories of intention, ed. P. D. Zelazo, J. W. Astington \& D. R. Olson. Erlbaum. [aJIMC, TJE]
Povinelli, D. J. \& Eddy, T. J. (1996) What young chimpanzees know about seeing. Monographs of the Society for Research in Child Development 61 (2, Serial No. 247). [rJIMC, TJE]

Putnam, H. (1988) Representation and reality. MIT Press. [aJIMC]

Quartz, S. R. \& Sejnowsky, T. J. (1997) A neural basis of cognitive development: A neuroconstructivist manifesto. Behavioral and Brain Sciences 20:537-56. [VM]

Rabbitt, P. (1997) Introduction: Methodologies and models in the study of executive function. In: Methodology of frontal and executive function, ed. P. Rabbitt. Psychology Press. [rJIMC]

Racine, T. (2004) Wittgenstein's internalistic logic and children's theories of mind. In: Social interaction and the development of knowledge, ed. J. I. M. Carpendale \& U. Müller. Erlbaum. [aJIMC]

Raver, C. C. \& Leadbeater, B. J. (1993) The problem of the other in research on theory of mind and social development. Human Development 36:350-62. [aJIMC, APC]

Reddy, V. (1991) Playing with others' expectations: Teasing and mucking about in the first year. In: Natural theories of mind: Evolution, development and simulation of everyday mindreading, ed. A. Whiten, pp. 143-58. Blackwell. [arJIMC]

(2003) On being the object of attention: Implications for self-other consciousness. Trends in Cognitive Science 7:397-402. [rJIMC]

Reese, E. (2002) Social factors in the development of autobiographical memory: The state of the art. Social Development 11:124-42. [KN, ASW-A]

Reese, E., Haden, C. A. \& Fivush, R. (1993) Mother-child conversations about the past: Relationships of style and memory over time. Cognitive Development 8:403-30. [ASW-A]

Repacholi, B. M. \& Gopnik, A. (1997) Early reasoning about desires: Evidence from 14- and 18-month-olds. Developmental Psychology 33:12-21. [TR]

Rochat, P. \& Striano, T. (2002) Who's in the mirror? Self-other discrimination in specular images by four- and nine-month-old infants. Child Development 73:35-46. [rJIMC, TR]

Rogoff, B. (1990) Apprenticeship in thinking: Cognitive development in social context. Oxford University Press. [RAT]

(1997) Evaluating development in the process of participation: Theory, methods, and practice building on each other. In: Change and development: Issues of theory, method, and application, ed. E. Amsel \& K. A. Renninger. Erlbaum. [aJIMC]

(1998) Cognition as a collaborative process. In: Handbook of child psychology, vol. 2, 5th edition, ed. D Kuhn \& R. S. Siegler. Wiley. [aJIMC]

Rogoff, B., Chavajay, P. \& Matusov, E. (1993) Questioning assumptions about culture and individuals. Behavioral and Brain Sciences 16:533-34. [JWA]

Rogoff, B., Mistry, J., Goncu, A. \& Mosier, C. (1993) Guided participation in cultural activity by toddlers and caregivers. Monographs of the Society for Research in Child Development 58 (Issue No. 8):v-179. [CW-B]

Rorty, R. (1980) Philosophy and the mirror of nature. Blackwell. [APC]

Roth, D. \& Leslie, A. M. (1998) Solving belief problems: Toward a task analysis Cognition 66:1-31. [TPG]

Rothbart, M. K., Ahadi, S. A. \& Evans, D. E. (2000) Temperament and personality: Origins and outcomes. Journal of Personality and Social Psychology 78:12235. $[\mathrm{PF}]$

Rowe, A. D., Bullock, P. R., Polkey, C. E. \& Morris, R. G. (2001) "Theory of mind" impairments and their relationship to executive functioning following frontal lobe excisions. Brain 124(Pt 3):600-16. [PF]

Rowlands, M. (1999) The body in mind. Cambridge University Press. [APC]

Ruffman, T. (1996) Do children understand the mind by means of simulation or a theory? Evidence from their understanding of inference. Mind and Language 11:388-414. [TR]

(2000) Nonverbal theory of mind: Is it important, is it implicit, is it simulation, is it relevant to autism. In: Minds in the making: Essays in honor of David R. Olson, ed. J. W. Astington, pp. 250-66. Blackwell. [aJIMC, TR]

Ruffman, T., Garnham, W., Import, A. \& Connolly, D. (2001a) Does eye direction indicate implicit sensitivity to false belief?: Charting transitions in knowledge. Journal of Experimental Child Psychology 80:201-24. [TR]

Ruffman, T., Garnham, W. \& Rideout, P. (2001b) Social understanding in autism: Eye gaze as a measure of core insights. Journal of Child Psychology and Psychiatry 42:1083-94. [TR]

Ruffman, T., Perner, J., Naito, M., Parkin, L. \& Clements, W. A. (1998) Older (but not younger) siblings facilitate false belief understanding. Developmental Psychology 34:161-74. [aJIMC]

Ruffman, T., Perner, J. \& Parkin, L. (1999) How parenting style affects false belief understanding. Social Development 8:395-411. [aJIMC, EM]

Ruffman, T., Slade, L. \& Crowe, E. (2002) The relation between children's and mothers' mental state language and theory-of-mind understanding. Child Development 73:734-51. [aJIMC, JMJ, TR, DKS]

Russell, J. (1987) Rule-following, mental models, and the development view. In: Meaning and the growth of understanding: Wittgenstein's significance for developmental psychology, ed. M. Chapman \& R. A. Dixon. Springer-Verlag. [aJIMC] 


\section{References/Carpendale \& Lewis: Constructing an understanding of mind}

(1992) The theory theory: So good they named it twice? Cognitive Development 7:485-519. [aJIMC]

(1996) Agency: Its role in mental development. Erlbaum/Taylor \& Francis/ Psychology Press. [arJIMC, SH]

Sabbagh, M. A. \& Callanan, M. A. (1998) Metarepresentation in action: 3-, 4-, and 5-year-olds' developing theories of mind in parent-child conversations. Developmental Psychology 34:491-502. [aJIMC, CAM]

Savage-Rumbaugh, S. E., Murphy, J., Sevcik, R. A., Brakke, K. E., Williams, S. L. \& Rumbaugh, D. M. (1993) Language comprehension in ape and child. Monographs of the Society for Research in Child Development 58 (Serial No. 233). [aJIMC]

Scarr, S. \& McCartney, K. (1983) How people make their own environments: A theory of genotype $\rightarrow$ environment effects. Child Development 54:424-35. $[\mathrm{SH}]$

Scarr, S. \& McCartney, K. (1984) How people make their own environments: A theory of genotype $\rightarrow$ environment effects. Annual Progress in Child Psychiatry and Child Development 98-118. [SH]

Schaffer, H. R., ed. (1977) Studies in mother-infant interaction. Academic Press. [arJIMC]

(1984a) Studies in mother-infant interaction, 2nd edition. Academic Press. [CW-B] (1984b) The child's entry into a social world. Academic Press. [aJIMC]

Schegloff, E. A. (1992) To Searle on conversation: A note in return. In: (On) Searle on conversation, ed. H. Parret \& J. Verschueren, pp. 113-28. John Benjamins. [rJIMC]

Scheler, M. (1954) The nature of sympathy, trans. P. Heath. Archon Books. (Original work published 1913.) [aJIMC]

Scholl, B. J. \& Leslie, A. M. (1999) Modularity, development and theory of mind. Mind and Language 14:131-53. [TPG, MS]

(2001) Minds, modules, and meta-analysis. Commentary on "Meta-analysis of theory-of-mind development: The truth about false belief." Child Development 72:696-701. [aJIMC, TPG, OML]

Schore, A. N. (1994) Affect regulation and the origins of self: The neurobiology of emotional development. Erlbaum. [HS]

(2000) Attachment and the regulation of the right brain. Attachment and Human Development 2:23-47.

(2001) The effects of early relational trauma on right brain development, affect regulation, and infant mental health. Infant Mental Health Journal 22:201-69. [HS]

(2003) Affect regulation and the repair of the self. Norton. [PF

Schultz, D., Izard, C. E., Ackerman, B. P. \& Youngstrom, E. A. (2001) Emotion knowledge in economically disadvantaged children: Self-regulatory antecedents and relations to social difficulties and withdrawal. Development and Psychopathology 13:53-67. [CEI]

Schultz, D., Izard, C. E. \& Bear, G. G. (in press) Emotionality, emotion information processing, and aggression. Development and Psychopathology [CEI]

Searle, J. R. (1992) Conversation. In: (On) Searle on conversation, ed. H. Parret \& J. Verschueren, pp. 7-29. John Benjamins. [rJIMC]

Selman, R. L. (1980) The growth of interpersonal understanding. Academic Press. [rJIMC, OML]

Selman, R. L., Schorin, M. Z., Stone, C. R. \& Phelps, E. (1983) A naturalistic study of children's social understanding. Developmental Psychology 19:82-102. [aJIMC]

Shankar, S. (1996) The conflict between Wittgenstein and Quine on the nature of language and cognition and its implications for constraint theory. In: Wittgenstein and Quine, ed. R. L. Arrington \& H.-J. Glock, pp. 212-51. Routledge. [MHB]

Shantz, C. U. \& Hartup, W. W. (1992) Conflict in child and adolescent development. Cambridge University Press. [NH]

Shatz, M. (1994) Theory of mind and the development of social-linguistic intelligence in early childhood. In: Children's early understanding of mind, ed. C. Lewis \& P. Mitchell, pp. 311-29. Erlbaum. [JWA]

Shaw, L. K. (1999) The development of the meanings of "think" and "know" through conversation. Unpublished doctoral dissertation, City University of New York Graduate Center, New York. [KN]

Shimmon, K., Lewis, C. \& Francis, B. (2003) Unity and diversity in executive skills and false belief: A longitudinal analysis. Paper presented at the biennial meeting of the Society for Research in Child Development, Tampa, April 2003. [rJIMC]

Shotter, J. (1978) The cultural context of communication studies: Theoretical and methodological issues. In: Action, gesture and symbol: The emergence of language, ed. A. Lock. Academic Press. [aJIMC]

Shweder, R., Goodnow, J. J., Hatano, G., LeVine, R., Markus, H. \& Miller, P. (1998) Cultural psychology of human development: One mind, many mentalities. In: Handbook of child psychology, vol. 1, 5th edition, ed. R. Lerner. Wiley. [MS]

Siegal, M. (1997) Knowing children: Experiments in conversation and cognition, 2nd edition. Psychology Press. [MS]
(2002) The science of childhood. In: The cognitive basis of science, ed. P. Carruthers, S. Stich \& M. Siegal. Cambridge University Press. [MS]

Siegal, M. \& Varley, R. (2002) Neural systems involved in "theory of mind." Nature Reviews Neuroscience 3(6):463-71. [PF, MS]

Sinha, C. (1999) Grounding, mapping and acts of meaning. In: Cognitive linguistics: Foundations, scope and methodology, ed. T. Janssen \& G. Redeker. Mouton de Gruyter. [aJIMC]

Skuse, D. (2003) Fear recognition and the neural basis of social cognition. Child and Adolescent Mental Health 8:50-60. [HS]

Slade, A., Grienenberger, J., Bernbach, E., Levy, D. \& Locker, A. (2001) Maternal reflective functioning: Considering the transmission gap. Paper presented at the Biennial Meeting of the Society for Research in Child Development, Minneapolis, MN, April 2001. [PF]

Slaughter, V. \& Gopnik, A. (1996) Conceptual coherence in the child's theory of mind: Training children to understand belief. Child Development 67:2967-88. [DKS]

Smiley, P. A. (2001) Intention understanding and partner-sensitive behaviors in young children's peer interactions. Social Development 10:330-54. [SZ]

Smith, L. (1993) Necessary knowledge: Piagetian perspectives on constructivism. Erlbaum. [rJIMC]

(1995) Introduction. In: Sociological studies by J. Piaget, ed. L. Smith. Routledge. [aJIMC]

(1996) The social construction of rational understanding. In: Piaget-Vygotsky: The social genesis of thought, ed. A. Tryphon \& J. Vonèche, pp. 107-23. Psychology Press. [rJIMC]

(2002) Reasoning by mathematical induction in children's arithmetic. Pergamon. [LS]

(2003) From epistemology to psychology in the development of knowledge. In: Reductionism and the development of knowledge, ed. T. Brown. \& L. Smith. Erlbaum. [LS]

Soffer, G. (1999) The other as alter ego: A genetic approach. Husserl Studies 15:151-66. [aJIMC]

Sokol, B. W. \& Chandler, M. J. (2003) Taking agency seriously in the theories-ofmind enterprise: Exploring children's understanding of interpretation and intention. British Journal of Educational Psychology Monograph Series II (No. 2: Development and Motivation):125-36. [rJIMC]

Sommerville, J. A. \& Woodward, A. L. (in press) Pulling out the intentional structure of action: The relation between action processing and action production in infancy. Cognition. [CW-B]

Spelke, E. (1994) Initial knowledge: Six suggestions. Cognition 50:431-45. [APC]

Steele, H. \& Steele, M. (1998) Attachment and psychoanalysis: Time for a reunion. Social Development 7:92-119. [HS]

(1999) Psychoanalytic views about development. In: Exploring developmental psychology, ed. D. Messer \& S. Millar, pp. 263-83. Francis Arnold. [HS] (2002) State of the art: Attachment. The Psychologist 15:518-22. [HS] (2003) Clinical uses of the Adult Attachment Interview. In: Attachment theory and the psychoanalytic process, ed. M. Marrone \& M. Cortina, pp 107-26. Whurr. [HS]

Steele, H., Steele, M. \& Fonagy, P. (1996) Associations among attachment classifications of mothers, fathers and their infants. Child Development 67:541-55. [HS]

Steele, H., Steele, M., Croft, C. \& Fonagy, P. (1999) Infant-mother attachment at one year predicts children's understanding of mixed emotions at six years. Social Development 8:161-78. [aJIMC, HS]

Steele, M., Steele, H. \& Johansson, M. (2002) Maternal predictors of children's social cognition: An attachment perspective. Journal of Child Psychology and Psychiatry 43:189-98. [HS]

Stern, D. (1977) The first relationship. Harvard University Press. [rJIMC] (1985) The interpersonal world of the infant. Basic Books.

Stich, S. \& Nichols, S. (1992) Folk psychology: Simulation of tacit theory? Mind and Language 7:35-71. [aJIMC]

Stuss, D. T., Gallup, G. G., Jr. \& Alexander, M. P. (2001) The frontal lobes are necessary for "theory of mind." Brain 124(Pt 2):279-86. [PF]

Subbotsky, E. (1993) The birth of personality: The development of independent and moral behavior in preschool children. Harvester Wheatsheaf. [OC]

Suchman, L. (1987) Plans and situated action: The problem of human-machine interaction. Cambridge University Press. [rJIMC]

Sullivan, K., Zaitchik, D. \& Tager-Flusberg, H. (1994) Preschoolers can attribute second-order beliefs. Developmental Psychology 30:395-402. [RB]

Sullivan, M. W., Lewis, M. \& Alessandri, S. M. (1992) Cross-age stability in emotional expressions during learning and extinction. Developmental Psychology 28:58-63. [CEI]

Sullivan, M. W. \& Lewis, M. (2003) Contextual determinants of anger and other negative expressions in young infants. Developmental Psychology 39:693-705. [CEI]

Summerfield, D. M. (1996) Fitting versus tracking: Wittgenstein on representation. In: The Cambridge companion to Wittgenstein, ed. H. Sluga \& D. G. Stern, pp. 100-38. Cambridge University Press. [MHB] 
Symons, D. K. (2004) Mental state discourse and theory of mind: Internalisation of self-other understanding within a social-cognitive framework. Developmental Review 24:159-88. [DKS]

Symons, D. K. \& Clark, S. E. (2000) A longitudinal study of mother-child relationships and theory of mind during the preschool period. Social Development 9:3-23. [aJIMC]

Symons, D. K., Peterson, C. C., Slaughter, V., Roche, J. \& Doyle, J. (in press) Theory of mind and mental state discourse during book reading and storytelling tasks. British Journal of Developmental Psychology [DKS]

Tager-Flusberg, H. (2002) How language facilitates the acquisition of false belief in children with autism. Paper presented at the International Conference, "Why Language Matters for Theory of Mind," Toronto, Canada, April 2002. [JWA]

Target, M., Shmueli-Goetz, Y. \& Fonagy, P. (in press) Attachment representations in school-age children: The early development of the child attachment interview (CAI). Journal of Infant, Child and Adolescent Psychotherapy. $[\mathrm{PF}]$

Taylor, C. (1985) Human agency and language: Philosophical papers, vol. 1. Cambridge University Press. [BWS]

ter Hark, M. (1990) Beyond the inner and the outer: Wittgenstein's philosophy of psychology. Kluwer Academic. [aJIMC]

Thelen, E., Schöner, G., Scheier, C. \& Smith, L. B. (2001) The dynamics of embodiment: A field theory of infant perseverative reaching. Behavioral and Brain Sciences 24:1-86. [arJIMC]

Thelen, E. \& Smith, L. B. (1994) A dynamic systems approach to the development of cognition and action. MIT Press. [aJIMC, OML]

Thomas, M. \& Karmiloff-Smith, A. (2002) Modelling typical and atypical development: Computational constraints on the mechanism of change. In: Blackwell handbook of cognitive developmental psychology, ed. U. Goswami. Blackwell. [SRL]

Thompson, R. A. (1998) Early sociopersonality development. In: Handbook of child psychology, vol. 3. Social, emotional, and personality development, 5th edition, ed. N. Eisenberg, pp. 25-104. Wiley. [RAT]

(2000) The legacy of early attachments. Child Development 71:145-52. [RAT]

Thompson, R. A., Laible, D. J. \& Ontai, L. L. (2003) Early understanding of emotion, morality, and the self: Developing a working model. In: Advances in child development and behavior, vol. 31, ed. R. V. Kail. Academic. [RAT]

Thompson, R. A. \& Raikes, H. A. (2003) Toward the next quarter-century: Conceptual and methodological challenges for attachment theory. Development and Psychopathology 15:691-718. [RAT]

Tomasello, M. (1995a) Joint attention as social cognition. In: Joint attention: Its origins and role in development, ed. C. Moore \& P. J. Dunham. Erlbaum. [aJIMC]

(1995b) Language is Not an instinct. Cognitive Development 10:131-56. [aJIMC] (1998) The return of constructions. Journal of Child Language 25:431-47. [rJIMC, NB]

(1999a) Having intentions, understanding intentions, and understanding communicative intentions. In: Developing theories of intention, ed. P. D. Zelazo, J. W. Astington \& D. R. Olson. Erlbaum. [aJIMC]

(1999b) The cultural origins of human cognition. Harvard University Press. [arJIMC]

(2001) Perceiving intentions and learning words in the second year of life. In: Language development: The essential readings, ed. M. Tomasello \& E. Bates, pp. 111-28. Harvard University Press. [rJIMC]

(2003) Constructing a language: A usage-based theory of language acquisition Harvard University Press. [rJIMC]

Tomasello, M. \& Camaioni, L. (1997) A comparison of the gestural communication of apes and human infants. Human Development 40:7-24. [aJIMC]

Tomasello, M., Kruger, A. C. \& Ratner, H. H. (1993) Cultural learning. Behavioral and Brain Sciences 16:495-552. [aJIMC, SZ]

Tomkins, S. S. (1962) Affect, imagery, consciousness, vol. I. The positive affects. Springer. [CEI]

Towse, J. N., Redbond, J., Housten-Price, C. M. T. \& Cook, S. (2000) Understanding the dimensional change card sort: Perspectives from task success and failure. Cognitive Development 15:347-65. [rJIMC]

Trevarthen, C. (1979) Communication and cooperation in early infancy: A description of primary intersubjectivity. In: Before speech: The beginning of interpersonal communication, ed. M. M. Bullowa. Cambridge University Press. [aJIMC]

(2003) Infant psychology is an evolving culture. Human Development 46:23346. [HS]

Trevarthen, C. \& Hubley, P. (1978) Secondary intersubjectivity: Confidence, confiding and acts of meaning in the first year. In: Action, gesture and symbol: The emergence of language, ed. A. Lock. Academic Press. [aJIMC]

Tronick, E., Als, A. \& Brazelton, T. B. (1977) Mutuality in mother-infant interaction. Journal of Communication 27:74-79. [CEI]

Tronick, E. \& Weinberg, M. K. (1997) Depressed mothers and infants: Failure to form dyadic states of consciousness. In: Postpartum depression and child development, ed. L. Murray \& P. Cooper. Guilford Press. [HS]
Turnbull, W. (2003) Language in action: Psychological models of conversation. Psychology Press. [arJIMC]

Turnbull, W. \& Carpendale, J. I. M. (1999a) A social pragmatic model of talk: Implications for research on the development of children's social understanding. Human Development 42:328-55. [arJIMC, CAM]

(1999b) Locating meaning in interaction, not in the brain. Behavioral and Brain Sciences 22:304-305. [aJIMC]

(2001) Talk and social understanding. Early Education and Development 12:455-77. [aJIMC]

Valsiner, J. (1998) The development of the concept of development: Historical and epistemological perspectives. In: Handbook of child psychology, vol. 1, 5th edition: Theoretical models of human development, ed. R. M. Lerner (W. Damon, Ed.-in-Chief), pp. 189-232. Wiley. [rJIMC]

van IJzendoorn, M. H. (1995) Adult attachment representations, parental responsiveness, and infant attachment: A meta-analysis on the predictive validity of the adult attachment interview. Psychological Bulletin 117:387403. [PF]

van IJzendoorn, M. H. \& Bakersman-Kranenburg, M. J. (1996) Attachment representations in mothers, fathers, adolescents, and clinical groups: A metaanalytic search for normative data. Journal of Consulting and Clinical Psychology 64:8-21. [HS]

Varouxaki, A., Freeman, N. H., Peters, D. \& Lewis, C. (1999) Inference neglect and inference denial. British Journal of Developmental Psychology 17:48399. [aJIMC, MS]

Vinden, P. G. (1996) Junin Quechua children's understanding of mind. Child Development 67:1701-16. [aJIMC]

(1999) Children's understanding of mind and emotion: A multi-cultural study. Cognition and Emotion 13:19-48. [aJIMC]

(2000) Making up my mind: Learning the culture of OISE and Olson. In: Minds in the making: Essays in honor of David R. Olson, ed. J. W. Astington, pp. 2942. Blackwell. [PGV]

(2001) Parenting attitudes and children's understanding of mind: A comparison of Korean American and Anglo-American families. Cognitive Development 16:793-809. [aJIMC]

Vinden, P. \& Astington, J. (2000) Culture and understanding other minds. In: Understanding other minds: Perspectives from developmental cognitive neuroscience, 2nd edition, ed. S. Baron-Cohen, H. Tager-Flusberg \& D. Cohen, pp. 503-19. Oxford University Press. [PGV]

von Wright, G. H. (1963) Norm and action. Routledge. [LS]

Vygotsky, L. S. (1930/1978) Mind in society: The development of higher psychological processes. Harvard University Press. (Original work published 1930, 1933, and 1935.) [APC, arJIMC, CF, DKS]

(1986) Thought and language. MIT Press. (Original work published 1934.) [aJIMC, DKS]

(1934/1987) Thinking and speech. In: The collected works of L. S. Vygotsky, vol. 1, ed. R. W. Rieber \& A. S. Carton, pp. 37-285. Plenum Press. (Original work published 1934.) [CF]

(1931/1997) Genesis of higher mental functions. In: The Collected Works of L. S. Vygotsky, vol. 4, ed. R. W. Rieber, pp. 97-119. Plenum Press. (Original work published 1931.) [CF]

(1998) The collected works of L. S. Vygotsky, vol. 5, Child psychology. Plenum Press. [aJIMC]

(1999) Tool and sign in the development of the child. In: The collected works of L. S. Vygotsky, vol. 6, ed. R. W. Rieber, pp. 3-65. Kluwer. (Original work published in 1930.) [OC]

Walden, T. \& Ogan, T. (1988) The development of social referencing. Child Development 59:1230-40. [aJIMC]

Walker-Andrews, A. S. (1997) Infants' perception of expressive behaviors: Differentiation of multimodal information. Psychological Bulletin 121:43756. [ASW-A]

Walker-Andrews, A. S. \& Kahana-Kalman, R. (1999) The understanding of pretence across the second year of life. British Journal of Developmental Psychology 17:523-36. [ASW-A]

Wallis, P. \& Steele, H. (2001) Attachment representations in adolescence: Further evidence from psychiatric residential settings. Attachment and Human Development 3:259-68. [HS]

Watson, J. S. (1972) Smiling, cooing, and 'the game.' Merrill Palmer Quarterly 18:323-40. [rJIMC]

Weinberg, M. K. \& Tronick, E. Z. (1994) Beyond the face: An empirical study of infant affective configurations of facial, vocal, gestural, and regulatory behaviors. Child Development 65:1503-15. [CEI]

Weizman, Z. O. \& Snow, C. E. (2001) Lexical input as related to children's vocabulary acquisition: Effects of sophisticated exposure and support for meaning. Developmental Psychology 37:265-79. [TR]

Wellman, H. M. (1990) The child's theory of mind. MIT Press. [KB, aJIMC, TR]

(1998) Culture, variation, and levels of analysis in folk pyschologies: Comment on Lillard (1998) Psychological Bulletin 123:33-36. [aJIMC]

Wellman, H. M., Cross, D. \& Watson, J. (2001) Meta-analysis of theory of mind 
References/Carpendale \& Lewis: Constructing an understanding of mind

development: The truth about false belief. Child Development 72:655-84. [aJIMC, JMJ, PM]

Wellman, H. M. \& Gelman, S. A. (1998) Knowledge acquisition in foundational domains. In: Handbook of child psychology, vol. 2, Cognition, perception, and language, 5th edition, ed. D. Kuhn \& R. Siegler, pp. 523-73. Wiley. [DEM]

Welsh, M. C., Pennington, B. F. \& Grossier, D. B. (1991) A normativedevelopmental study of executive function: A window on prefrontal function in children. Developmental Neuropsychology 7:131-49. [SH]

Wenner, J. A. \& Bauer, P. J. (1999) Bringing order to the arbitrary: One- to twoyear olds' recall of event sequences. Infant Behavior and Development 22:585-90. [CW-B]

Werner, H. \& Kaplan, B. (1963) Symbol formation. Wiley. [aJIMC]

Williams, M. (1999) Wittgenstein, mind and meaning: Toward a social conception of mind. Routledge. [aJIMC]

Wimmer, H. \& Hartl, M. (1991) Against the Cartesian view on mind: Young children's difficulty with own false beliefs. British Journal of Developmental Psychology 9:125-38. [PM]

Winnicott, D. W. (1965) The capacity to be alone. The maturational processes and the facilitating environment: Studies in the theory of emotional development. Hogarth Press. [HS]

(1971) Playing and reality. Penguin. [JMJ]

Wittgenstein, L. (1953/1968) Philosophical investigations. Blackwell. [arJIMC, PG, JS]

(1958) Philosophical investigations, trans. G. E. M. Anscombe. Macmillan. [MHB, DEM]

(1969) On certainty. Harper \& Row. [MHB, rJIMC]

(1980) Culture and value. Blackwell. [rJIMC]

(1981a) Zettel. Blackwell. [aJIMC]

(1981b) Zettel, 2nd edition, ed. G. E. M. Anscombe \& G. H. V. Wright. Blackwell. [JS

Woodfield, A. (1996) Which theoretical concepts do children use? Philosophical Papers 25:1-20. [aJIMC]

Woodward, A. L. (1998) Infants selectively encode the goal object of an actor's reach. Cognition 69:1-34. [CW-B]
(1999) Infants' ability to distinguish between purposeful and non-purposeful behaviors. Infant Behavior and Development 22:145-60. [CW-B]

(2003) Infants' developing understanding of the link between looker and object. Developmental Science 6:297-312. [CW-B]

Woodward, A. L. \& Guajardo, J. J. (2002) Infants' understanding of the point gesture as an object-directed action. Cognitive Development 17:1061-84. [CW-B]

Woodward, A. L. \& Sommerville, J. A. (2000) Twelve-month-old infants interpret action in context. Psychological Science 11:73-7. [CW-B]

Woolfe, T., Want, S. C. \& Siegal, M. (2002) Signposts to development: Theory of mind in deaf children. Child Development 73:768-78. [aJIMC]

Yasui, M. (2003) Oh great! You really helped me! Social cognition and irony comprehension in young children. Poster presented at the biennial meeting of the Society for Research in Child Development, Tampa, April. [rJIMC]

Youngblade, L. M. \& Dunn, J. (1995) Individual differences in young children’s pretend play with mothers and siblings: Links to relationships and understanding other people's feelings and beliefs. Child Development 66:1472-92. [aJIMC]

Yuill, N. \& Pearson, A. (1998) The development of bases for trait attribution: Children's understanding of traits as causal mechanisms based on desire. Developmental Psychology 34:574-687. [RB]

Zelazo, P. D., Carter, A., Reznick, J. S. \& Frye, D. (1997) Early development of executive function: A problem-solving framework. Review of General Psychology 1:1-29. [SH]

Zelazo, P. D. \& Frye, D. (1997) Cognitive complexity and control: A theory of the development of deliberate reasoning and intentional action. In: Language structure, discourse, and the access to consciousness, ed. M. Stamenov, pp. 113-53. John Benjamins. [CAM]

Zerwas, S. \& Brownell, C. A. (2003) Partners in pretense: Contributions of mothers and peers to early social pretend play. Poster session presented at the biennial meeting of the Society for Research in Child Development, Tampa, FL, April 2003. [SZ]

Zimmer, C. (2003) How the mind reads other minds. Science 300:1079-80. [CEI] 